\title{
Catalytic Synthesis of Conjugated Azopolymers from Aromatic Diazides
}

\section{Supporting Information}

John M. Andjaba, Christopher J. Rybak, Zhiyang Wang, Jianheng Ling, Jianguo Mei*, and Christopher Uyeda*

Department of Chemistry, Purdue University, 560 Oval Dr., West Lafayette, IN 47907, USA

*Correspondence: cuyeda@purdue.edu, jgmei@purdue.edu

1. General Information

2. Synthesis of Organoazides

S4

3. Reaction Optimization Studies

S21

4. Azopolymer Synthesis and Characterization

$\mathrm{S} 22$

Copolymerization Experiments

$\mathrm{S} 27$

5. Endcapping Experiments

S29

6. Lewis Acid Experiments

S33

7. Cyclic Voltammetry

S41

8. Photoswitching Experiments

$\mathrm{S} 50$

9. Thermogravimetric Analysis

S52

10. Emission spectra

S56

11. NMR Spectra

S57

12. UV-Vis Spectra

S103

13. IR Spectra

14. High Temperature Gel Permeation Chromatography (HT-GPC) Data

16. DFT Studies 


\section{General Information}

General Considerations. All manipulations were carried out using standard Schlenk or glovebox techniques under an atmosphere of $\mathrm{N}_{2}$. Solvents were dried and degassed by passing through a column of activated alumina and sparging with Ar gas. Deuterated solvents were purchased from Cambridge Isotope Laboratories, Inc., degassed, and stored over activated 3 Å molecular sieves prior to use. All other reagents and starting materials were purchased from commercial vendors and used without further purification unless otherwise noted. Liquid reagents were degassed and stored over activated 3 A molecular sieves prior to use. The $\left({ }^{i-P r} \mathrm{NDI}\right) \mathrm{Ni}_{2}\left(\mathrm{C}_{6} \mathrm{H}_{6}\right)$ complex (1), was prepared according to previously reported procedures. ${ }^{1}$

Physical Methods. ${ }^{1} \mathrm{H}$ NMR spectra were collected at room temperature on a Bruker Avance-III 800, Bruker AV-III-400-HD, Bruker DRX $500 \mathrm{MHz}$, or Varian Inova300 spectrometer. ${ }^{1} \mathrm{H}$ and ${ }^{13} \mathrm{C}$ NMR spectra are reported in parts per million relative to tetramethylsilane, using the residual solvent resonances as an internal standard. UV-vis measurements were acquired on an Agilent Cary 6000i UVVis-nIR spectrophotometer using a 1-cm two-window quartz cuvette. High-resolution mass data were obtained using a 6320 Ion Trap MS system. GPC data were obtained using a EcoSEC HLC-8321GPC/HT with a refractive index (RI) detector at $50^{\circ} \mathrm{C}$ or a TOSOH ECOSEC HLC-8320 GPC run at $180{ }^{\circ} \mathrm{C}$. IR data were collected on a Thermo Nicolet 6700 FT-IR spectrometer with an MCT-A detector and a $\mathrm{KBr}$ beam splitter with a range of $800-4500 \mathrm{~cm}^{-1}$. Emission spectra were collected using an Edinburgh Instruments FLS980 Steady State Fluorescence Spectrometer with a xenon lamp. Thermogravimetric analysis was collected using a TA Instrument Q50 TGA instrument with a heating rate of $20^{\circ} \mathrm{C} \cdot \mathrm{min}-1$ under an $\mathrm{N}_{2}$ atmosphere.

Spectroelectrochemistry Experiments. Spectroelectrochemistry data for 10 were obtained using a CHI 660E Potentiostat and a Flame Vis-NIR Fiber Optic Spectrometer Ocean Optics spectrometer. Electrochemical measurements of $\mathbf{1 5}$ were performed using a Gamry Interface 1000 Potentiostat. Cyclic voltammograms were acquired using a glassy carbon working electrode ( $3 \mathrm{~mm}$ diameter disk) under an atmosphere of $\mathrm{N}_{2}$. The potentials were internally referenced to the reversible $\mathrm{Fc} / \mathrm{Fc}^{+}$couple.

Photoswitching Experiments. An in-house irradiation setup was built using a TENMA 72-2685 power supply, a 3D printed holder for a 1-cm UV-Vis cuvette bearing 3 unmounted LEDs. Two light sources were used: $395 \mathrm{~nm}(6 \mathrm{~mW}$ at $20 \mathrm{~mA})$ and $555 \mathrm{~nm}(1 \mathrm{~mW}$ at $20 \mathrm{~mA})$ from Thorlabs, Newton, NJ, USA. The irradiance is $18 \mathrm{~mW} / \mathrm{cm}^{2}$ for the $395 \mathrm{~nm}$ LED unit and $3 \mathrm{~mW} / \mathrm{cm}^{2}$ for the $555 \mathrm{~nm}$ LED unit.

X-Ray Crystallography. Single Crystal XRD Data Collection. A single crystal of 17a was coated with a trace of fomblin oil, affixed onto a MiTeGen micromesh mount and transferred to the goniometer head of a Bruker Quest diffractometer with kappa geometry, an I- $\mu$-S microsource X-ray tube, laterally graded multilayer (Goebel) mirror single crystal for monochromatization, a Photon3 CMOS area detector and an Oxford Cryosystems low temperature device. Examination and data collection were performed with $\mathrm{Cu}$ Ka radiation $(\lambda=1.54178 \AA$ A $)$ at $100 \mathrm{~K}$. Data were collected, reflections were indexed and processed, and the files scaled and corrected for absorption using APEX3. ${ }^{2}$ 
The space group was assigned and the structure solved by direct methods using XPREP within the SHELXTL suite of programs ${ }^{3}$ from an integration using only the major domain. The structure was refined using the hklf 5 routine by full matrix least squares against $\mathrm{F}^{2}$ with all reflections of component 1 (including the overlapping ones), using Shelxl2016 ${ }^{4}$ using the graphical interface Shelxle ${ }^{5}$ resulting in a BASF value of $0.392(1)$.

$\mathrm{H}$ atoms were positioned geometrically and constrained to ride on their parent atoms, with carbon hydrogen bond distances of $0.95 \AA$ for aromatic C-H, 1.00, 0.99 and $0.98 \AA$ for aliphatic C- $\mathrm{H}_{1} \mathrm{CH}_{2}$ and $\mathrm{CH}_{3}$ moieties, and $0.84 \AA$ A for $\mathrm{O}-\mathrm{H}$ bonds, respectively. Methyl $\mathrm{H}$ and hydroxyl atoms were allowed to rotate but not to tip to best fit the experimental electron density. $\mathrm{U}_{\text {iso }}(\mathrm{H})$ values were set to a multiple of $\mathrm{U}_{\text {eq }}(\mathrm{C})$ with 1.5 for $\mathrm{CH}_{3}$, and 1.2 for $\mathrm{C}-\mathrm{H}$ units, respectively. Additional data collection and refinement details, including description of disorder and/or twinning (where present) can be found in Section 13.

Complete crystallographic data, in CIF format, have been deposited with the Cambridge Crystallographic Data Centre. CCDC 2054149 contains the supplementary crystallographic data for this paper. These data can be obtained free of charge from The Cambridge Crystallographic Data Centre via www.ccdc.cam.ac.uk/data_request/cif. 


\section{Synthesis of Organoazides}

SAFETY NOTE: Organoazides are high energy molecules, and they can be thermally unstable and shock sensitive. In order to minimize explosion hazards, care should be taken to avoid organoazides with low C-to- $\mathrm{N}$ ratios (generally $\mathrm{C}$-to- $\mathrm{N}$ ratios $>3$ are recommended), limit scale to the extent possible, and store organoazides in solution. We did not encounter any specific safety concerns with the organoazides used in these studies. TGA studies were carried out using diazides $\mathbf{S 1 0}$ and $\mathbf{2}$.

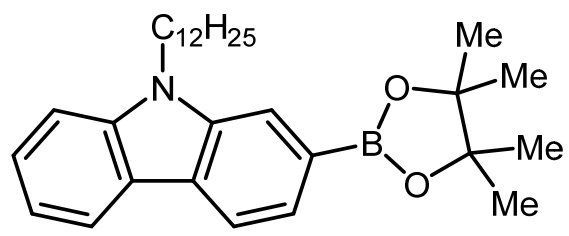

9-dodecyl-2-(4,4,5,5-tetramethyl-1,3,2-dioxaborolan-2-yl)-9H-carbazole (S1). This procedure is based on a modified literature procedure. ${ }^{6}$ In an $\mathrm{N}_{2}$ filled glovebox, a Schlenk tube was charged with 2-bromo-9-dodecyl-9H-carbazole 7 , (0.50 g, $1.2 \mathrm{mmol}, 1.0$ equiv), $\mathrm{Pd}$ (dppf) $\mathrm{Cl}_{2}$ (0.16 g, $0.22 \mathrm{mmol}, 0.18$ equiv), bis(pinacolato)diboron ( $0.37 \mathrm{~g}, 1.5 \mathrm{mmol}, 1.2$ equiv), KOAc ( $1.4 \mathrm{~g}, 15 \mathrm{mmol}, 12$ equiv), and 1,4dioxane $(33 \mathrm{~mL})$. The reaction vessel was sealed, removed from the glovebox, and heated at $80{ }^{\circ} \mathrm{C}$ for $16 \mathrm{~h}$. After cooling to room temperature, the reaction mixture was filtered through celite, and the filtrate was concentrated under reduced pressure. The residue was purified by column chromatography $\left(\mathrm{SiO}_{2}, 30 \% \mathrm{CH}_{2} \mathrm{Cl}_{2} /\right.$ hexanes, $\left.\mathrm{R}_{\mathrm{f}}=0.4\right)$. The product was isolated as a colorless oil $(0.32$ g, $57 \%$ yield).

${ }^{1} \mathrm{H}$ NMR $\left(500 \mathrm{MHz}, \mathrm{CDCl}_{3}\right) \delta 8.17-8.11(\mathrm{~m}, 2 \mathrm{H}), 7.91(\mathrm{~s}, 1 \mathrm{H}), 7.73(\mathrm{~d}, J=7.8 \mathrm{~Hz}, 1 \mathrm{H}), 7.50(\mathrm{t}, J=7.6 \mathrm{~Hz}$, $1 \mathrm{H}), 7.43(\mathrm{~d}, J=8.2 \mathrm{~Hz}, 1 \mathrm{H}), 7.27-7.22(\mathrm{~m}, 1 \mathrm{H}), 4.36(\mathrm{t}, J=7.4 \mathrm{~Hz}, 2 \mathrm{H}), 1.95-1.86(\mathrm{~m}, 2 \mathrm{H}), 1.43(\mathrm{~s}$, $12 \mathrm{H}), 1.28(\mathrm{~m}, 18 \mathrm{H}), 0.91(\mathrm{t}, J=7.0 \mathrm{~Hz}, 3 \mathrm{H})$.

${ }^{13} \mathrm{C}\left\{{ }^{1} \mathrm{H}\right\}$ NMR $\left(126 \mathrm{MHz}, \mathrm{CDCl}_{3}\right) \delta 141.0,140.0,126.2,125.4,125.1,124.9,122.6,120.8,119.6,118.7$, $115.1,108.9,83.8,43.0,32.0,29.6,29.1,27.3,25.2,25.0,24.9,22.7,14.1$.

HRMS (APCI): calcd for $\mathrm{C}_{30} \mathrm{H}_{45} \mathrm{NO}_{2} \mathrm{~B}^{10}[\mathrm{M}+\mathrm{H}]^{+}: 461.3574$; found: 461.3562 


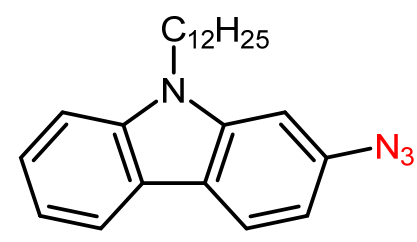

2-azido-9-dodecyl-9H-carbazole (4b). This procedure is based on a modified literature procedure..$^{8} \mathrm{NaN}_{3}\left(0.068 \mathrm{~g}, 1.0 \mathrm{mmol}, 1.5\right.$ equiv) and $\mathrm{Cu}(\mathrm{OAc})_{2}(0.013 \mathrm{~g}, 0.069 \mathrm{mmol}, 0.10$ equiv) were added to a solution of $\mathbf{S} \mathbf{1}\left(0.32 \mathrm{~g}, 0.69 \mathrm{mmol}, 1.0\right.$ equiv) in a mixture of $\mathrm{CHCl}_{3}(5.7 \mathrm{~mL})$ and $\mathrm{MeOH}(5.7$ $\mathrm{mL}$ ). The reaction was heated at $55^{\circ} \mathrm{C}$ and stirred under air for $24 \mathrm{~h}$. After cooling to room temperature, the reaction mixture was filtered and the filtrate was concentrated under reduced pressure. The residue was purified by column chromatography $\left(\mathrm{SiO}_{2}, 100 \%\right.$ hexanes, $\left.\mathrm{R}_{\mathrm{f}}=0.8\right)$. The product was isolated as an off-white solid ( $0.18 \mathrm{~g}, 70 \%$ yield).

${ }^{1} \mathrm{H}$ NMR $\left(800 \mathrm{MHz}, \mathrm{CDCl}_{3}\right) \delta 8.05(\mathrm{~d}, \mathrm{~J}=8.0 \mathrm{~Hz}, 2 \mathrm{H}), 7.46(\mathrm{t}, \mathrm{J}=7.6 \mathrm{~Hz}, 1 \mathrm{H}), 7.40(\mathrm{~d}, \mathrm{~J}=8.1 \mathrm{~Hz}, 1 \mathrm{H}), 7.25$ $(\mathrm{t}, \mathrm{J}=7.3 \mathrm{~Hz}, 1 \mathrm{H}), 7.01(\mathrm{~s}, 1 \mathrm{H}), 6.95(\mathrm{~d}, 1 \mathrm{H}), 4.26(\mathrm{t}, \mathrm{J}=7.3 \mathrm{~Hz}, 2 \mathrm{H}), 1.90-1.84(\mathrm{~m}, 2 \mathrm{H}), 1.39-1.24(\mathrm{~m}$, $18 \mathrm{H}), 0.89(\mathrm{t}, \mathrm{J}=7.2 \mathrm{~Hz}, 3 \mathrm{H})$.

${ }^{13} \mathrm{C}\left\{{ }^{1} \mathrm{H}\right\}$ NMR $\left(201 \mathrm{MHz}, \mathrm{CDCl}_{3}\right) \delta 141.3,140.8,137.7,125.5,122.5,121.4,120.3,120.0,119.2,110.2$, $108.7,99.0,43.1,31.8,29.5,29.5,29.4,29.3,29.3,28.8,27.2,22.6,14.1$.

HRMS (APCI): calcd for $\mathrm{C}_{24} \mathrm{H}_{32} \mathrm{~N}_{4}[\mathrm{M}+\mathrm{H}]^{+}: 377.2700$; found: 377.2704 


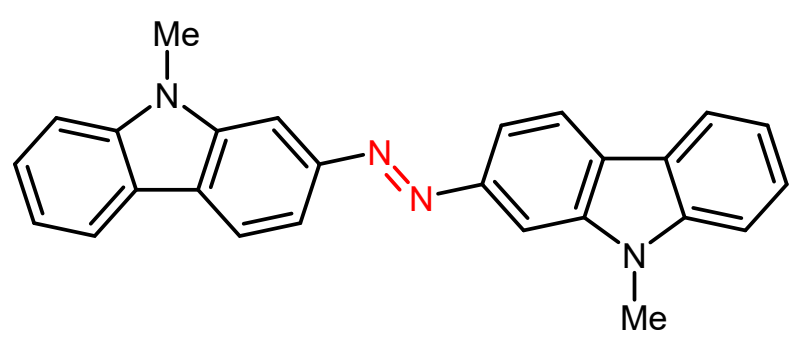

(E)-1,2-bis(9-methyl-9H-carbazol-2-yl)diazene (5a). This procedure is based on a modified literature procedure. ${ }^{9} \mathrm{In}$ an $\mathrm{N}_{2}$ filled glovebox, a 20 - $\mathrm{mL}$ vial was charged with $4 \mathbf{4 a}(0.044 \mathrm{~g}, 0.20 \mathrm{mmol}$, 1.0 equiv), toluene ( $4.0 \mathrm{~mL}$ ), and a magnetic stir bar. While stirring, $1(7.0 \mathrm{mg}, 0.010 \mathrm{mmol}, 5 \mathrm{~mol} \%)$ dissolved in toluene $(1.0 \mathrm{~mL})$ was added to the reaction vial. The vial was sealed, and the reaction mixture was stirred at room temperature. After $2 \mathrm{~h}$, the reaction vial was opened to air, and the crude mixture was directly loaded onto a column $\left(\mathrm{SiO}_{2}, 5 \% \mathrm{EtOAc} /\right.$ hexanes, $\left.\mathrm{R}_{\mathrm{f}}=0.3\right)$ for purification. The product was isolated as an orange solid. Run 1: $0.033 \mathrm{~g}, 44 \%$ yield; Run 2: $0.035 \mathrm{~g}$, $44 \%$ yield.

${ }^{1} \mathrm{H} \mathrm{NMR}\left(400 \mathrm{MHz}, \mathrm{CDCl}_{3}\right) \delta 8.22(\mathrm{~d}, \mathrm{~J}=8.3 \mathrm{~Hz}, 2 \mathrm{H}), 8.14(\mathrm{~d}, \mathrm{~J}=7.8 \mathrm{~Hz}, 2 \mathrm{H}), 8.07(\mathrm{~s}, 2 \mathrm{H}), 7.97(\mathrm{~d}, \mathrm{~J}=8.0$ $\mathrm{Hz}, 2 \mathrm{H}), 7.53(\mathrm{~m}, 2 \mathrm{H}), 7.44$ (d, J = 8.2 Hz, 2H), $7.30-7.27$ (m, 2H), 3.97 (s, 6H).

${ }^{13} \mathrm{C}\left\{{ }^{1} \mathrm{H}\right\}$ NMR $\left(201 \mathrm{MHz}, \mathrm{CDCl}_{3}\right) \delta 151.3,142.5,141.4,126.6,125.1,122.4,120.9,120.6,119.4,115.3$, $108.7,102.8,29.3$.

HRMS (APCI): calcd for $\mathrm{C}_{26} \mathrm{H}_{21} \mathrm{~N}_{4}[\mathrm{M}+\mathrm{H}]^{+}: 389.1761$; found: 389.1765 


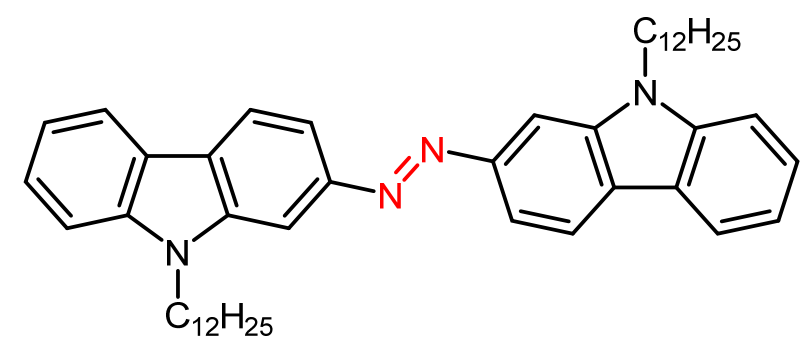

(E)-1,2-bis(9-dodecyl-9H-carbazol-2-yl)diazene (5b). This procedure is based on a modified literature procedure. ${ }^{9}$ In an $\mathrm{N}_{2}$ filled glovebox, a 20 -mL vial was charged with $4 \mathbf{b}(0.075 \mathrm{~g}, 0.20 \mathrm{mmol}$, 1.0 equiv), toluene ( $4.0 \mathrm{~mL}$ ), and a magnetic stir bar. While stirring, $1(7.0 \mathrm{mg}, 0.010 \mathrm{mmol}, 5 \mathrm{~mol} \%)$ dissolved in toluene $(1.0 \mathrm{~mL})$ was added to the reaction vial. The vial was sealed, and the reaction mixture was stirred at room temperature. After $2 \mathrm{~h}$, the reaction vial was opened to air, and the crude mixture was directly loaded onto a column $\left(\mathrm{SiO}_{2}, 2 \% \mathrm{EtOAc} /\right.$ hexanes, $\left.\mathrm{Rf}_{\mathrm{f}}=0.2\right)$ for purification. The product was isolated as a yellow solid. Run 1: $0.066 \mathrm{~g}$, 95\% yield; Run 2: $0.066 \mathrm{~g}$, 95\% yield.

${ }^{1} \mathrm{H}$ NMR (400 MHz, CDCl 3 ) $\delta 8.23(\mathrm{~d}, J=8.3 \mathrm{~Hz}, 2 \mathrm{H}), 8.16(\mathrm{~d}, J=7.8 \mathrm{~Hz}, 2 \mathrm{H}), 8.09(\mathrm{~d}, J=1.6 \mathrm{~Hz}, 2 \mathrm{H}), 7.98$ $(\mathrm{d}, J=8.3 \mathrm{~Hz}, 2 \mathrm{H}), 7.57-7.49(\mathrm{~m}, 2 \mathrm{H}), 7.45(\mathrm{~d}, J=8.2 \mathrm{~Hz}, 2 \mathrm{H}), 7.28(\mathrm{t}, J=7.3 \mathrm{~Hz}, 2 \mathrm{H}), 4.41(\mathrm{t}, J=7.3 \mathrm{~Hz}$, $4 \mathrm{H}), 1.96(\mathrm{p}, J=7.4 \mathrm{~Hz}, 4 \mathrm{H}), 1.50-1.42(\mathrm{~m}, 4 \mathrm{H}), 1.41-1.34(\mathrm{~m}, 4 \mathrm{H}), 1.33-1.22(\mathrm{~m}, 28 \mathrm{H}), 0.89(\mathrm{t}, J=$ $6.8 \mathrm{~Hz}, 6 \mathrm{H})$.

${ }^{13} \mathrm{C}\left\{{ }^{1} \mathrm{H}\right\}$ NMR (101 MHz, $\left.\mathrm{CDCl}_{3}\right) \delta 151.2,141.8,140.7,126.4,125.0,122.4,120.8,120.5,119.2,114.1$, $108.9,104.0,43.2,31.8,29.6,29.5,29.4,29.3,29.0,27.3,22.6,14.1$.

UV-Vis-NIR ( $\left.{ }_{6} \mathrm{H}_{5} \mathrm{Cl}, 0.143 \mathrm{mM}\right): \lambda_{\max }, \mathrm{nm}\left(\varepsilon, \mathrm{M}^{-1} \mathrm{~cm}^{-1}\right): 302$ (1800), 398 (4900).

HRMS (APCI): calcd for $\mathrm{C}_{48} \mathrm{H}_{65} \mathrm{~N}_{4}[\mathrm{M}+\mathrm{H}]^{+}: 697.5204$; found: 697.5207 


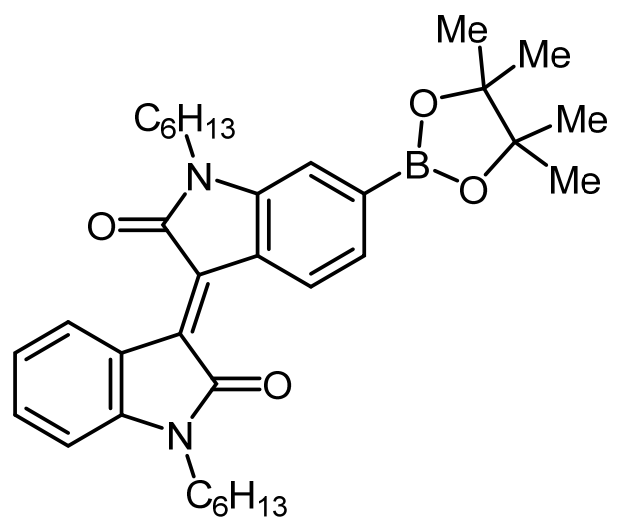

(E)-1,1'-dihexyl-6-(4,4,5,5-tetramethyl-1,3,2-dioxaborolan-2-yl)-[3,3'-biindolinylidene]2,2'-dione (S2). This procedure is based on a modified literature procedure. ${ }^{6}$ In an $\mathrm{N}_{2}$ filled glovebox, a Schlenk tube was charged with (E)-6-bromo-1,1'-dihexyl-[3,3'-biindolinylidene]-2,2'-dione ${ }^{10}$ (0.16 g, $0.31 \mathrm{mmol}, 1.0$ equiv), $\mathrm{Pd}(\mathrm{dppf}) \mathrm{Cl}_{2}(0.041 \mathrm{~g}, 0.057 \mathrm{mmol}, 0.18$ equiv), bis(pinacolato)diboron $(0.12 \mathrm{~g}$, $0.47 \mathrm{mmol}, 1.5$ equiv), KOAc ( $0.092 \mathrm{~g}, 0.94 \mathrm{mmol}, 3.0$ equiv), and 1,4-dioxane $(6.2 \mathrm{~mL})$. The reaction vessel was sealed, removed from the glovebox, and heated at $80^{\circ} \mathrm{C}$ for $16 \mathrm{~h}$. After cooling to room temperature, the reaction mixture was filtered, and the filtrate was concentrated under reduced pressure. The residue was purified by column chromatography $\left(\mathrm{SiO}_{2}, 10 \% \mathrm{Et}_{2} \mathrm{O} /\right.$ hexanes, $\left.\mathrm{R}_{\mathrm{f}}=0.2\right)$. The product was isolated as a red solid ( $0.14 \mathrm{~g}$, 81\% yield).

${ }^{1} \mathrm{H}$ NMR (400 MHz, CDCl 3 ) $\delta 9.18(\mathrm{~d}, 1 \mathrm{H}), 9.14(\mathrm{~d}, J=7.9 \mathrm{~Hz}, 1 \mathrm{H}), 7.49(\mathrm{~d}, J=7.9,1.0 \mathrm{~Hz}, 1 \mathrm{H}), 7.34(\mathrm{t}$, $1 \mathrm{H}), 7.16(\mathrm{~s}, 1 \mathrm{H}), 7.03(\mathrm{t}, 1 \mathrm{H}), 6.77(\mathrm{~d}, J=7.8 \mathrm{~Hz}, 1 \mathrm{H}), 3.78(\mathrm{p}, J=15.2,7.4 \mathrm{~Hz}, 4 \mathrm{H}), 1.76-1.66(\mathrm{~m}, 4 \mathrm{H})$, $1.37(\mathrm{~s}, 12 \mathrm{H}), 1.35-1.22(\mathrm{~m}, 12 \mathrm{H}), 0.91-0.86(\mathrm{~m}, 6 \mathrm{H})$.

${ }^{13} \mathrm{C}\left\{{ }^{1} \mathrm{H}\right\}$ NMR $\left(101 \mathrm{MHz}, \mathrm{CDCl}_{3}\right) \delta 167.7,167.6,144.8,143.8,134.2,133.4,132.4,130.0,128.9,128.7$, 124.2, 122.0, 121.6, 113.0, 107.8, 84.0, 40.0, 39.9, 31.4, 27.4, 27.3, 26.6, 26.6, 24.8, 22.5, 13.9, 13.9.

HRMS (APCI): calcd for $\mathrm{C}_{34} \mathrm{H}_{45} \mathrm{~B}^{10} \mathrm{~N}_{2} \mathrm{O}_{4}[\mathrm{M}+\mathrm{H}]^{+}: 556.3581$; found: 556.3571 


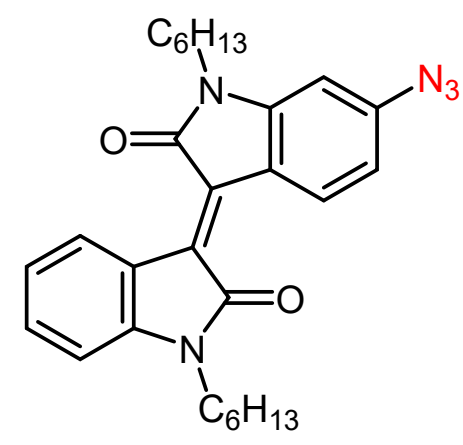

(E)-6-azido-1,1'-dihexyl-[3,3'-biindolinylidene]-2,2'-dione (S3). This procedure is based on a modified literature procedure. ${ }^{8} \mathrm{NaN}_{3}\left(0.060 \mathrm{~g}, 0.93 \mathrm{mmol}, 1.5\right.$ equiv) and $\mathrm{Cu}(\mathrm{OAc})_{2}(0.011 \mathrm{~g}, 0.062$ mmol, 0.10 equiv) were added to a solution of $\mathbf{S} \mathbf{1}\left(0.34 \mathrm{~g}, 0.62 \mathrm{mmol}, 1.0\right.$ equiv) in a mixture of $\mathrm{CHCl}_{3}$ $(5.0 \mathrm{~mL})$ and $\mathrm{MeOH}(5.0 \mathrm{~mL})$. The reaction was heated at $55^{\circ} \mathrm{C}$ and stirred under air for $24 \mathrm{~h}$. After cooling to room temperature, the reaction mixture was filtered, and the filtrate was concentrated under reduced pressure. The residue was purified by column chromatography $\left(\mathrm{SiO}_{2}, 10 \%\right.$ $\mathrm{Et}_{2} \mathrm{O} /$ hexanes, $\left.\mathrm{R}_{\mathrm{f}}=0.3\right)$. The product was isolated as a deep red solid. $(0.14 \mathrm{~g}, 48 \%$ yield).

${ }^{1} \mathrm{H}$ NMR $\left(500 \mathrm{MHz}, \mathrm{CDCl}_{3}\right) \delta 9.23(\mathrm{~d}, J=8.6 \mathrm{~Hz}, 1 \mathrm{H}), 9.14(\mathrm{~d}, J=8.0 \mathrm{~Hz}, 1 \mathrm{H}), 7.33(\mathrm{t}, 1 \mathrm{H}), 7.03(\mathrm{t}, J=7.8$ $\mathrm{Hz}, 1 \mathrm{H}), 6.77(\mathrm{~d}, J=7.8 \mathrm{~Hz}, 1 \mathrm{H}), 6.68(\mathrm{dd}, J=8.6,2.2 \mathrm{~Hz}, 1 \mathrm{H}), 6.36(\mathrm{~d}, J=2.2 \mathrm{~Hz}, 1 \mathrm{H}), 3.79-3.70(\mathrm{~m}$, $4 \mathrm{H}), 1.68(\mathrm{~m}, 4 \mathrm{H}), 1.44-1.35(\mathrm{~m}, 4 \mathrm{H}), 1.35-1.27(\mathrm{~m}, 8 \mathrm{H}), 0.91-0.85(\mathrm{~m}, 6 \mathrm{H})$.

${ }^{13} \mathrm{C}\left\{{ }^{1} \mathrm{H}\right\}$ NMR $\left(126 \mathrm{MHz}, \mathrm{CDCl}_{3}\right) \delta 168.2,167.9,146.5,144.6,143.8,132.5,132.2,131.6,129.8,122.1$, $121.7,118.7,111.9,107.9,99.1,98.9,40.2,40.1,31.5,27.5,26.7,22.5,14.0$.

HRMS (APCI): calcd for $\mathrm{C}_{28} \mathrm{H}_{33} \mathrm{~N}_{5} \mathrm{O}_{2}[\mathrm{M}+\mathrm{H}]^{+}: 472.2707$ found: 472.2717 
<smiles>CCCCCCCCCCCCCCCCCCCCCCCCCCCCCCC</smiles>

9-(pentacosan-13-yl)-2,7-bis(4,4,5,5-tetramethyl-1,3,2-dioxaborolan-2-yl)-9H-carbazole (S4). This procedure is based on a modified literature procedure. ${ }^{6}$ In an $\mathrm{N}_{2}$ filled glovebox, a Schlenk tube was charged with 2,7-dibromo-9-(pentacosan-13-yl)-9H-carbazole ${ }^{11}$, (1.0 g, $1.5 \mathrm{mmol}, 1.0$ equiv), $\mathrm{Pd}(\mathrm{dppf}) \mathrm{Cl}_{2}$ (0.39 mg, $0.53 \mathrm{mmol}, 0.35$ equiv), bis(pinacolato)diboron ( $0.90 \mathrm{mg}, 3.6 \mathrm{mmol}, 2.4$ equiv), KOAc (1.7 g, $18 \mathrm{mmol}, 12$ equiv), and 1,4-dioxane ( $40 \mathrm{~mL}$ ). The reaction vessel was sealed, removed from the glovebox, and heated at $80^{\circ} \mathrm{C}$ for $16 \mathrm{~h}$. After cooling to room temperature, the reaction mixture was filtered, and the filtrate was concentrated under reduced pressure. The residue was purified by column chromatography $\left(\mathrm{SiO}_{2}, 50 \% \mathrm{CH}_{2} \mathrm{Cl}_{2}\right.$ /hexanes, $\left.\mathrm{Rf}_{\mathrm{f}}=0.4\right)$. The product was isolated as a yellow oil ( $0.64 \mathrm{~g}$, $56 \%$ yield).

${ }^{1} \mathrm{H} \mathrm{NMR}\left(800 \mathrm{MHz}, \mathrm{CDCl}_{3}\right) \delta 8.14(\mathrm{~d}, \mathrm{~J}=24.3,2 \mathrm{H}), 8.04(\mathrm{~s}, 1 \mathrm{H}), 7.90(\mathrm{~s}, 1 \mathrm{H}), 7.74-7.63(\mathrm{~m}, 2 \mathrm{H}), 4.75-$ $4.67(\mathrm{~m}, 1 \mathrm{H}), 2.40-2.30(\mathrm{~m}, 2 \mathrm{H}), 2.00-1.93(\mathrm{~m}, 2 \mathrm{H}), 1.41(\mathrm{~s}, 24 \mathrm{H}), 1.33-1.09(\mathrm{~m}, 38 \mathrm{H}), 1.02-0.97$ $(\mathrm{m}, 2 \mathrm{H}), 0.88(\mathrm{t}, \mathrm{J}=7.2 \mathrm{~Hz}, 6 \mathrm{H})$.

${ }^{13} \mathrm{C}\left\{{ }^{1} \mathrm{H}\right\}$ NMR $\left(201 \mathrm{MHz}, \mathrm{CDCl}_{3}\right) \delta 141.9,138.6,128.3,126.0,124.6,124.5,119.96,119.65,118.0,115.4$, 83.6, 56.3, 33.8, 31.8, 29.6, 29.5, 29.5, 29.4, 29.3, 29.3, 26.7, 24.9, 22.6, 14.0.

HRMS (APCI): calcd for $\mathrm{C}_{49} \mathrm{H}_{81} \mathrm{~B}^{10}{ }_{2} \mathrm{NO}_{4}[\mathrm{M}+\mathrm{H}]$ : 769.6461 ; found: 769.6446 


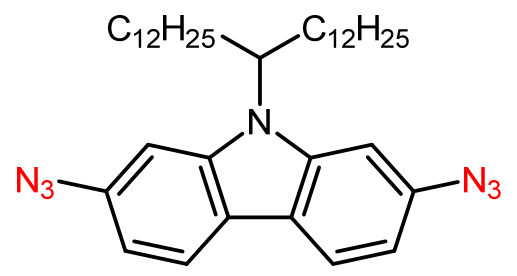

2,7-diazido-9-(pentacosan-13-yl)-9H-carbazole (2). This procedure is based on a modified literature procedure. ${ }^{8} \mathrm{NaN}_{3}\left(0.13 \mathrm{~g}, 2.0 \mathrm{mmol}, 3.0\right.$ equiv) and $\mathrm{Cu}(\mathrm{OAc})_{2}(0.024 \mathrm{~g}, 0.13 \mathrm{mmol}, 0.20$ equiv) were added to a solution of $\mathbf{S} \mathbf{4}\left(0.51 \mathrm{~g}, 0.66 \mathrm{mmol}, 1.0\right.$ equiv) in a mixture of $\mathrm{CHCl}_{3}(3.3 \mathrm{~mL})$ and $\mathrm{MeOH}(3.3 \mathrm{~mL})$. The reaction was heated at $55^{\circ} \mathrm{C}$ and stirred under air for $24 \mathrm{~h}$. After cooling to room temperature, the reaction mixture was filtered, and the filtrate was concentrated under reduced pressure. The residue was purified by column chromatography $\left(\mathrm{SiO}_{2}, 100 \%\right.$ hexanes, $\left.\mathrm{R}_{\mathrm{f}}=0.6\right)$. The product was isolated as a yellow oil ( $0.29 \mathrm{~g}, 73 \%$ yield).

${ }^{1} \mathrm{H}$ NMR $\left(800 \mathrm{MHz}, \mathrm{CDCl}_{3}\right) \delta 7.98(\mathrm{dd}, \mathrm{J}=28.7,8.3 \mathrm{~Hz}, 2 \mathrm{H}), 7.12(\mathrm{~s}, 1 \mathrm{H}), 6.97-6.90(\mathrm{~m}, 3 \mathrm{H}), 4.45-4.38$ $(\mathrm{m}, 1 \mathrm{H}), 2.25-2.14(\mathrm{~m}, 2 \mathrm{H}), 1.93-1.82(\mathrm{~m}, 2 \mathrm{H}), 1.30-1.08(\mathrm{~m}, 38 \mathrm{H}), 1.01-0.94(\mathrm{~m}, 2 \mathrm{H}), 0.87(\mathrm{t}, \mathrm{J}=$ $7.2 \mathrm{~Hz}, 6 \mathrm{H})$.

${ }^{13} \mathrm{C}\left\{{ }^{1} \mathrm{H}\right\}$ NMR $\left(201 \mathrm{MHz}, \mathrm{CDCl}_{3}\right) \delta 143.3,139.7,137.6,137.0,128.3,121.0,120.8,119.7,110.5,110.4$, $102.0,99.4,56.7,33.4,31.8,29.5,29.5,29.4,29.3,29.2,26.6,22.6,14.1$.

HRMS (APCI): calcd for $\mathrm{C}_{37} \mathrm{H}_{58} \mathrm{~N}_{7}$ [M + H]: 600.4748; found: 600.4752 


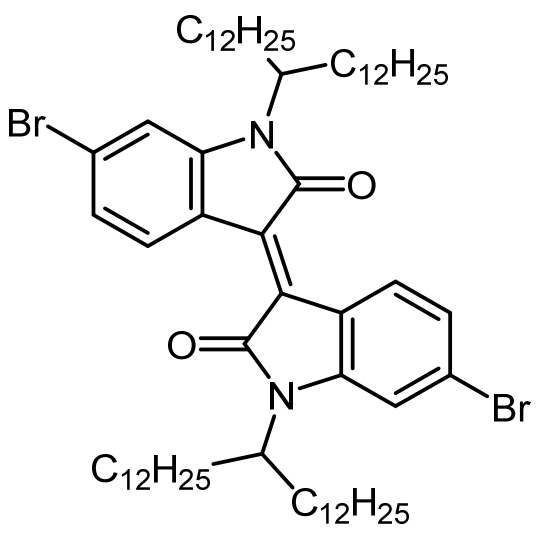

(E)-6,6'-dibromo-1,1'-di(pentacosan-13-yl)-[3,3'-biindolinylidene]-2,2'-dione (S5). This procedure is based on a modified literature procedure. ${ }^{12} 6,6^{\prime}$-dibromoisoindigo ${ }^{12}(0.42 \mathrm{~g}, 1.0 \mathrm{mmol}$, 1.0 equiv) and $\mathrm{Cs}_{2} \mathrm{CO}_{3}(0.98 \mathrm{~g}, 3.0 \mathrm{mmol}, 3.0$ equiv) were added to a reaction flask and sealed. The reaction vessel was sparged with $\mathrm{N}_{2}$ to remove air. Anhydrous $\mathrm{N}, \mathrm{N}$-Dimethylformamide (10 $\mathrm{mL}$ ) was added, and the mixture was heated at $70^{\circ} \mathrm{C}$. After 30 minutes of heating, pentacosan-13-yl 4methylbenzenesulfonate ${ }^{13}(1.2 \mathrm{~g}, 2.2 \mathrm{mmol}, 2.2$ equiv) dissolved in anhydrous $\mathrm{N}, \mathrm{N}-$ dimethylformamide $(10.0 \mathrm{~mL})$ was added, and the reaction was allowed to stir for $16 \mathrm{~h}$ at $70{ }^{\circ} \mathrm{C}$. After cooling to room temperature, the reaction mixture was filtered, and the filtrate was concentrated under reduced pressure. The residue was purified by column chromatography $\left(\mathrm{SiO}_{2}\right.$, hexanes, $\left.\mathrm{R}_{\mathrm{f}}=0.2\right)$. The product was isolated as a deep red solid ( $0.56 \mathrm{~g}, 50 \%$ yield).

${ }^{1} \mathrm{H}$ NMR (800 MHz, CDCl $)$ $\delta 9.06(\mathrm{~s}, 2 \mathrm{H}), 7.23-6.90(\mathrm{~m}, 4 \mathrm{H}), 4.64(\mathrm{~s}, 1 \mathrm{H}), 3.69(\mathrm{~s}, 1 \mathrm{H}), 2.26(\mathrm{~s}, 1 \mathrm{H})$, $1.92(\mathrm{~s}, 3 \mathrm{H}), 1.75(\mathrm{~m}, 4 \mathrm{H}), 1.38-1.13(\mathrm{~m}, 80 \mathrm{H}), 0.89(\mathrm{t}, J=7.2 \mathrm{~Hz}, 12 \mathrm{H})$.

${ }^{13} \mathrm{C}\left\{{ }^{1} \mathrm{H}\right\} \mathrm{NMR}\left(201 \mathrm{MHz}, \mathrm{CDCl}_{3}\right) \delta 168.1,144.6,130.9,126.4,124.7,120.5,113.1,52.5,31.9,31.9,29.6$, $29.6,29.5,29.5,29.4,29.3,26.6,22.6,14.1$.

HRMS (APCI): calcd for $\mathrm{C}_{66} \mathrm{H}_{109} \mathrm{Br}_{2} \mathrm{~N}_{2} \mathrm{O}_{2}[\mathrm{M}+\mathrm{H}]^{+}: 1119.6850$; found: 1119.6866 


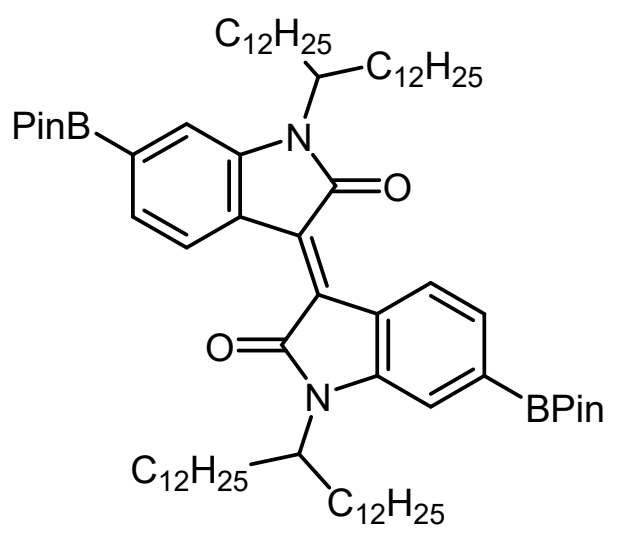

(E)-1,1'-di(pentacosan-13-yl)-6,6'-bis(4,4,5,5-tetramethyl-1,3,2-dioxaborolan-2-yl)-[3,3'biindolinylidene]-2,2'-dione (S6). This procedure is based on a modified literature procedure. ${ }^{6}$ In an $\mathrm{N}_{2}$ filled glovebox, a Schlenk tube was charged with $\mathbf{S 5}\left(0.56 \mathrm{~g}, 0.50 \mathrm{mmol}, 1.0\right.$ equiv), $\mathrm{Pd}(\mathrm{dppf}) \mathrm{Cl}_{2}$ ( $0.022 \mathrm{~g}, 0.030 \mathrm{mmol}, 0.060$ equiv), bis(pinacolato)diboron $(0.38 \mathrm{~g}, 1.5 \mathrm{mmol}, 3.0$ equiv), KOAc ( $0.29 \mathrm{~g}$, $3.0 \mathrm{mmol}, 6.0$ equiv), and 1,4-dioxane $(10.0 \mathrm{~mL})$. The reaction vessel was sealed, removed from the glovebox, and heated at $80^{\circ} \mathrm{C}$ for $16 \mathrm{~h}$. After cooling to room temperature, the reaction mixture was filtered, and the filtrate was concentrated under reduced pressure. The residue was purified by column chromatography $\left(\mathrm{SiO}_{2}, 20 \% \mathrm{CH}_{2} \mathrm{Cl}_{2} /\right.$ hexanes, $\left.\mathrm{Rf}=0.25\right)$. The product was isolated as a deep red solid $(0.49 \mathrm{~g}, 80 \%$ yield $)$.

${ }^{1} \mathrm{H} \mathrm{NMR}\left(400 \mathrm{MHz}, \mathrm{CDCl}_{3}\right) \delta 9.09(\mathrm{~s}, 2 \mathrm{H}), 7.45(\mathrm{~d}, J=8.0 \mathrm{~Hz}, 2 \mathrm{H}), 7.26(\mathrm{~s}, 3 \mathrm{H}), 4.64(\mathrm{~s}, 1 \mathrm{H}), 3.88(\mathrm{~s}, 1 \mathrm{H})$, $2.40-1.90(\mathrm{~m}, 4 \mathrm{H}), 1.82-1.66(\mathrm{~m}, 4 \mathrm{H}), 1.36(\mathrm{~s}, 24 \mathrm{H}), 1.32-1.15(\mathrm{~m}, 80 \mathrm{H}), 0.86(\mathrm{t}, J=7.0 \mathrm{~Hz}, 12 \mathrm{H})$.

${ }^{13} \mathrm{C}\left\{{ }^{1} \mathrm{H}\right\}$ NMR $\left(201 \mathrm{MHz}, \mathrm{CDCl}_{3}\right) \delta$ 168.0, 142.9, 133.6, 128.5, 124.3, 115.2, 83.9, 52.3, 32.0, 31.8, 29.6, 29.6, 29.5, 29.4, 29.3, 26.7, 24.8, 22.6, 14.1.

HRMS (APCI): calcd for $\mathrm{C}_{78} \mathrm{H}_{133} \mathrm{~B}^{10}{ }_{2} \mathrm{~N}_{2} \mathrm{O}_{4}[\mathrm{M}+\mathrm{H}]^{+}:$1215.0381; found: 1215.0417 


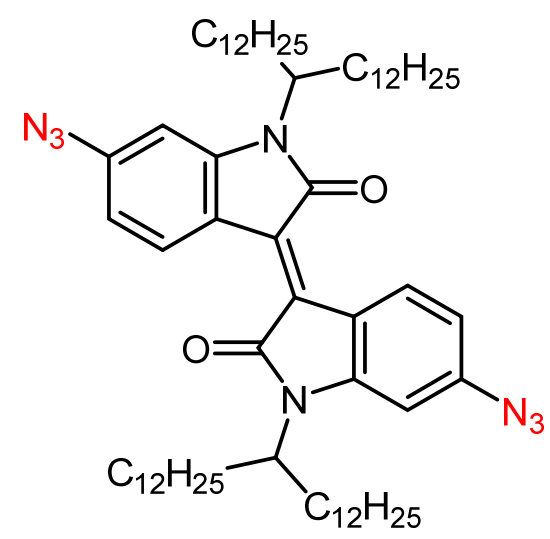

(E)-6,6'-diazido-1,1'-di(pentacosan-13-yl)-[3,3'-biindolinylidene]-2,2'-dione (S7). This procedure is based on a modified literature procedure. ${ }^{8} \mathrm{NaN}_{3}(0.036 \mathrm{~g}, 0.56 \mathrm{mmol}, 3.0$ equiv) and $\mathrm{Cu}(\mathrm{OAc})_{2}(7.0 \mathrm{mg}, 0.037 \mathrm{mmol}, 0.20 \mathrm{equiv})$ were added to a solution of $\mathbf{S} \mathbf{6}(0.23 \mathrm{~g}, 0.19 \mathrm{mmol}, 1.0$ equiv) in a mixture of $\mathrm{CHCl}_{3}(3.5 \mathrm{~mL})$ and $\mathrm{MeOH}(3.5 \mathrm{~mL})$. The reaction was heated at $55^{\circ} \mathrm{C}$ and stirred under air for $16 \mathrm{~h}$. After cooling to room temperature, the reaction mixture was filtered, and the filtrate was concentrated under reduced pressure. The residue was purified by column chromatography $\left(\mathrm{SiO}_{2}, 10 \% \mathrm{CH}_{2} \mathrm{Cl}_{2} /\right.$ hexanes, $\left.\mathrm{R}_{\mathrm{f}}=0.3\right)$. The product was isolated as a deep red solid (0.098 g, 50\% yield).

${ }^{1} \mathrm{H} \mathrm{NMR}\left(500 \mathrm{MHz}, \mathrm{CDCl}_{3}\right) \delta 9.17(\mathrm{~s}, 2 \mathrm{H}), 6.70(\mathrm{~d}, J=8.7 \mathrm{~Hz}, 2 \mathrm{H}), 6.55(\mathrm{~s}, 2 \mathrm{H}), 4.64(\mathrm{~s}, 1 \mathrm{H}), 3.68(\mathrm{~s}, 1 \mathrm{H})$, $2.26(\mathrm{~s}, 1 \mathrm{H}), 1.90(\mathrm{~s}, 3 \mathrm{H}), 1.78-1.67(\mathrm{~m}, 4 \mathrm{H}), 1.37-1.14(\mathrm{~m}, 80 \mathrm{H}), 0.87(\mathrm{t}, J=6.9 \mathrm{~Hz}, 12 \mathrm{H})$.

${ }^{13} \mathrm{C}\left\{{ }^{1} \mathrm{H}\right\}$ NMR $\left(126 \mathrm{MHz}, \mathrm{CDCl}_{3}\right) \delta 168.7,145.2,143.5,131.1,131.1,118.8,111.5,101.1,52.4,31.9,29.9$, 29.6, 29.6, 29.5, 29.4, 26.7, 23.0, 22.7, 14.1.

HRMS (APCI): calcd for $\mathrm{C}_{66} \mathrm{H}_{109} \mathrm{~N}_{8} \mathrm{O}_{2}[\mathrm{M}+\mathrm{H}]^{+}: 1045.8668$; found: 1045.8696 


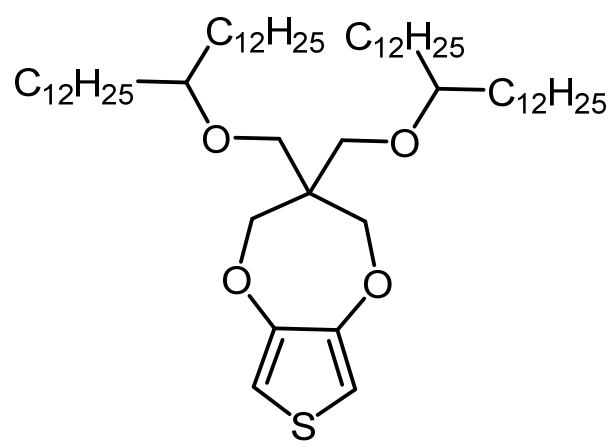

\section{3,3-bis((pentacosan-13-yloxy)methyl)-3,4-dihydro-2H-thieno[3,4-b][1,4]dioxepine (S8).} This procedure is based on a modified literature procedure. ${ }^{14}$ A round-bottom flask was charged with $\mathrm{NaH}\left(0.11 \mathrm{~g}, 4.4 \mathrm{mmol}, 3.0\right.$ equiv) and pentacosan-13-ol ${ }^{13}(1.2 \mathrm{~g}, 3.2 \mathrm{mmol}, 2.1$ equiv). The solution was sparged with $\mathrm{N}_{2}$ to remove air. Anhydrous $\mathrm{N}, \mathrm{N}$-dimethylformamide $(6.0 \mathrm{~mL})$ was added to the flask, and the reaction mixture was heated at $70{ }^{\circ} \mathrm{C}$. After $6 \mathrm{~h}, 3,3$-bis(bromomethyl)-3,4-dihydro- $2 \mathrm{H}$ thieno[3,4-b][1,4]dioxepine ${ }^{14}(0.50 \mathrm{~g}, 1.5 \mathrm{mmol}, 1.0$ equiv) was added as a solid, and the reaction mixture was allowed to stir at $70^{\circ} \mathrm{C}$ for $48 \mathrm{~h}$. After cooling to room temperature, the reaction mixture was added to $200 \mathrm{~mL}$ of sat. $\mathrm{NaCl}(\mathrm{aq})$. The crude product was extracted with three portions of diethyl ether, and the combined organic phases were washed with three portions of water. The organic phase was dried using $\mathrm{Na}_{2} \mathrm{SO}_{4}$ then concentrated under reduced pressure. The residue was purified by column chromatography $\left(\mathrm{SiO}_{2}, 15 \% \mathrm{CH}_{2} \mathrm{Cl}_{2} /\right.$ hexanes, $\left.\mathrm{Rf}=0.3\right)$. The product was isolated as a white solid (0.65 g, 48\% yield).

${ }^{1} \mathrm{H} \mathrm{NMR}\left(400 \mathrm{MHz}, \mathrm{CDCl}_{3}\right) \delta 6.43(\mathrm{~s}, 2 \mathrm{H}), 4.01(\mathrm{~s}, 4 \mathrm{H}), 3.50(\mathrm{~s}, 4 \mathrm{H}), 3.23-3.12(\mathrm{~m}, 2 \mathrm{H}), 1.50-1.36(\mathrm{~m}$, $8 \mathrm{H}), 1.26(\mathrm{~s}, 80 \mathrm{H}), 0.88(\mathrm{t}, J=6.7 \mathrm{~Hz}, 12 \mathrm{H})$.

${ }^{13} \mathrm{C}\left\{{ }^{1} \mathrm{H}\right\} \mathrm{NMR}\left(201 \mathrm{MHz}, \mathrm{CDCl}_{3}\right) \delta 149.7,104.7,67.7,47.8,34.1,33.6,31.9,29.9,29.7,29.6,29.6,29.3$, 25.3, 22.6, 22.3, 14.1, 14.0.

HRMS (APCI): calcd for $\mathrm{C}_{59} \mathrm{H}_{113} \mathrm{O} \mathrm{O}_{4} \mathrm{~S}[\mathrm{M}+\mathrm{H}]^{+}: 917.8354$; found: 917.8342 


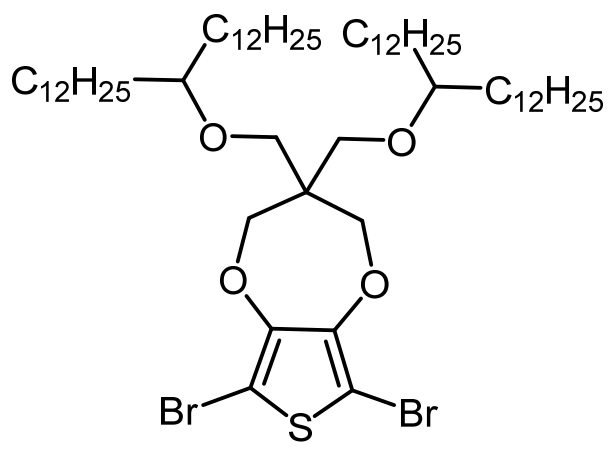

\section{6,8-dibromo-3,3-bis((pentacosan-13-yloxy)methyl)-3,4-dihydro-2H-thieno[3,4-}

b][1,4]dioxepine (S9). This procedure is based on a modified literature procedure. ${ }^{14} \mathrm{~A}$ round bottom flask was charged with $\mathbf{S 8}(0.30 \mathrm{~g}, 0.33 \mathrm{mmol}, 1.0$ equiv $)$ and NBS $(0.17 \mathrm{~g}, 0.98 \mathrm{mmol}, 3.0$ equiv). The flask was sparged with $\mathrm{N}_{2}$, and $\mathrm{CHCl}_{3}(6.0 \mathrm{~mL})$ was added under $\mathrm{N}_{2}$. The reaction was allowed to stir at room temperature for $16 \mathrm{~h}$. The reaction mixture was concentrated under reduced pressure, and the residue was purified by column chromatography $\left(\mathrm{SiO}_{2}, 10 \% \mathrm{CH}_{2} \mathrm{Cl}_{2} /\right.$ hexanes, $\left.\mathrm{Rf}_{\mathrm{f}}=0.4\right)$. The product was isolated as a white solid ( $0.33 \mathrm{~g}, 94 \%$ yield).

${ }^{1} \mathrm{H}$ NMR (500 MHz, CDCl 3$) \delta 4.09(\mathrm{~s}, 4 \mathrm{H}), 3.53(\mathrm{~s}, 4 \mathrm{H}), 3.21-3.15(\mathrm{~m}, 2 \mathrm{H}), 1.49-1.23(\mathrm{~m}, 88 \mathrm{H}), 0.88(\mathrm{t}$, $J=6.9 \mathrm{~Hz}, 12 \mathrm{H})$.

${ }^{13} \mathrm{C}\left\{{ }^{1} \mathrm{H}\right\} \mathrm{NMR}\left(126 \mathrm{MHz}, \mathrm{CDCl}_{3}\right) \delta 147.3,90.9,67.9,48.2,33.8,32.1,30.1,29.9,29.9,29.6,25.5,22.9$, 14.3 .

HRMS (APCI): calcd for $\mathrm{C}_{59} \mathrm{H}_{110} \mathrm{Br}_{2} \mathrm{O}_{4} \mathrm{~S}[\mathrm{M}+\mathrm{H}]^{+}: 1073.6564$; found: 1073.6551 


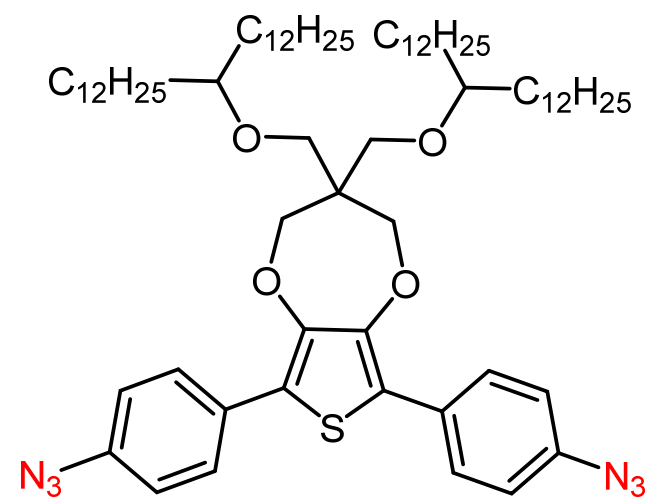

6,8-bis(4-azidophenyl)-3,3-bis((pentacosan-13-yloxy)methyl)-3,4-dihydro-2H-thieno[3,4b][1,4]dioxepine (S10). This procedure is based on a modified literature procedure. ${ }^{15}$ In an $\mathrm{N}_{2}$ filled glovebox, a Schlenk tube was charged with $\mathbf{S 9}$ ( $0.19 \mathrm{~g}, 0.18 \mathrm{mmol}, 1.0$ equiv), 2-(4-azidophenyl)4,4,5,5-tetramethyl-1,3,2-dioxaborolane ${ }^{16}(0.095 \mathrm{~g}, 0.39 \mathrm{mmol}, 2.2$ equiv), tris(omethoxyphenyl)phosphine ( $0.015 \mathrm{~g}, 0.042 \mathrm{mmol}, 0.24$ equiv), $\mathrm{K}_{3} \mathrm{PO}_{4}(0.23 \mathrm{~g}, 1.1 \mathrm{mmol}, 6.0$ equiv), $\mathrm{Pd}_{2}(\mathrm{dba})_{3}(0.019 \mathrm{~g}, 0.021 \mathrm{mmol}, 0.12$ equiv), Aliquat 336 (1 drop), and toluene (6.1 mL). The Schlenk tube was sealed and removed from the glovebox. Under $\mathrm{N}_{2}$, degassed $\mathrm{H}_{2} \mathrm{O}(0.90 \mathrm{~mL})$ was added. The reaction was allowed to stir at room temperature for $4 \mathrm{~h}$. The reaction mixture was then diluted with $\mathrm{CH}_{2} \mathrm{Cl}_{2}$ and washed with water $(2 \times 50 \mathrm{~mL})$. The organic phase was dried over $\mathrm{Na}_{2} \mathrm{SO}_{4}$, filtered, and concentrated under reduced pressure. The residue was purified by column chromatography $\left(\mathrm{SiO}_{2}\right.$, $20 \% \mathrm{CH}_{2} \mathrm{Cl}_{2} /$ hexanes, $\mathrm{R}_{\mathrm{f}}=0.4$ ). The product was isolated as an off-white solid. ( $0.12 \mathrm{~g}, 60 \%$ yield).

${ }^{1} \mathrm{H} \mathrm{NMR}\left(400 \mathrm{MHz}, \mathrm{CDCl}_{3}\right) \delta 7.72(\mathrm{~d}, J=8.8 \mathrm{~Hz}, 4 \mathrm{H}), 7.02(\mathrm{~d}, J=8.8 \mathrm{~Hz}, 4 \mathrm{H}), 4.16(\mathrm{~s}, 4 \mathrm{H}), 3.58(\mathrm{~s}, 4 \mathrm{H})$, $3.26-3.16(\mathrm{~m}, 2 \mathrm{H}), 1.43(\mathrm{~s}, 8 \mathrm{H}), 1.26(\mathrm{~s}, 80 \mathrm{H}), 0.87(\mathrm{t}, J=6.8 \mathrm{~Hz}, 12 \mathrm{H})$.

${ }^{13} \mathrm{C}\left\{{ }^{1} \mathrm{H}\right\}$ NMR $\left(201 \mathrm{MHz}, \mathrm{CDCl}_{3}\right) \delta 146.4,138.2,129.9,127.8,119.1,118.3,80.0,73.9,67.7,47.9,33.6$, $31.9,29.9,29.7,29.6,29.3,25.3,22.6,14.1$.

HRMS (APCI): calcd for $\mathrm{C}_{71} \mathrm{H}_{118} \mathrm{~N}_{6} \mathrm{O}_{4} \mathrm{~S}$ [M + H]+: 1151.9008; found: 1151.8990 


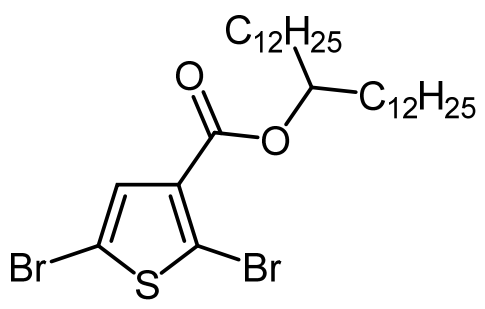

Pentacosan-13-yl 2,5-dibromothiophene-3-carboxylate (S11). This procedure is based on a modified literature procedure. ${ }^{17}$ A round-bottom flask was charged with 2,5-dibromothiophene-3carboxylic acid ${ }^{17}(0.37 \mathrm{~g}, 1.0 \mathrm{mmol}, 1.0$ equiv), 4-Dimethylaminopyridine $(0.37 \mathrm{~g}, 3.0 \mathrm{mmol}, 3.0$ equiv), pentacosan-13-ol ${ }^{13}$ (0.86 g, $3.0 \mathrm{mmol}, 3.0$ equiv), and N,N'-dicyclohexylcarbodiimide $(0.62 \mathrm{~g}, 3.0 \mathrm{mmol}$, 3.0 equiv). The reaction vessel was sparged with $\mathrm{N}_{2}$, and $\mathrm{CH}_{2} \mathrm{Cl}_{2}(10 \mathrm{~mL})$ was added to the reaction flask. The reaction was allowed to stir at room temperature for $48 \mathrm{~h}$. The resulting mixture was filtered through celite, and the filtrate was concentrated under reduced pressure. The residue was purified by column chromatography ( $\mathrm{SiO}_{2}, 10 \% \mathrm{Et}_{2} \mathrm{O} /$ hexanes). The product was isolated as a white semi-solid. (0.46 mg, 72\% yield).

${ }^{1} \mathrm{H}$ NMR (400 MHz, $\left.\mathrm{CDCl}_{3}\right) \delta 7.33(\mathrm{~s}, 1 \mathrm{H}), 5.13-5.01(\mathrm{~m}, 1 \mathrm{H}), 1.68-1.57(\mathrm{~m}, 4 \mathrm{H}), 1.26(\mathrm{~d}, \mathrm{~J}=6.5 \mathrm{~Hz}$, $40 \mathrm{H}), 0.88(\mathrm{~m}, 6 \mathrm{H})$.

${ }^{13} \mathrm{C}\left\{{ }^{1} \mathrm{H}\right\} \mathrm{NMR}\left(101 \mathrm{MHz}, \mathrm{CDCl}_{3}\right) \delta 160.7,132.5,131.9,118.9,111.3,76.0,34.8,34.2,32.1,31.8,29.8$, $29.8,29.7,29.7,29.5,28.4,25.5,22.9,22.8,20.8,14.3,11.6$.

HRMS (APCI): calcd for $\mathrm{C}_{30} \mathrm{H}_{52} \mathrm{Br}_{2} \mathrm{O}_{2} \mathrm{~S}[\mathrm{M}+\mathrm{H}]^{+}: 635.2128$; found: 635.2123 


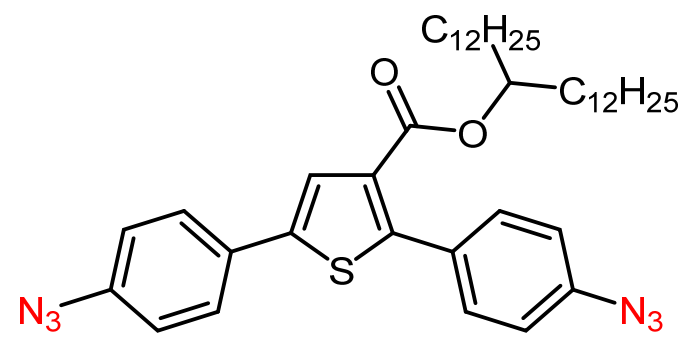

Pentacosan-13-yl 2,5-bis(4-azidophenyl)thiophene-3-carboxylate (S12). This procedure is based on a modified literature procedure..$^{15}$ In an $\mathrm{N}_{2}$ filled glovebox, a Schlenk tube was charged with S11 ( $0.20 \mathrm{~g}, 0.31 \mathrm{mmol}, 1.0$ equiv), 2-(4-azidophenyl)-4,4,5,5-tetramethyl-1,3,2-dioxaborolane ${ }^{16}(0.17$ g, $0.69 \mathrm{mmol}, 2.2$ equiv), tris(o-methoxyphenyl)phosphine $\left(0.027 \mathrm{~g}, 0.075 \mathrm{mmol}, 0.24\right.$ equiv), $\mathrm{K}_{3} \mathrm{PO}_{4}$ ( $0.40 \mathrm{~g}, 1.9 \mathrm{mmol}, 6.0$ equiv), $\mathrm{Pd}_{2}(\mathrm{dba})_{3}(0.035 \mathrm{~g}, 0.038 \mathrm{mmol}, 0.12$ equiv), Aliquat 336 (1 drop), and toluene $(11 \mathrm{~mL})$. The Schlenk tube was sealed and removed from the glovebox. Under $\mathrm{N}_{2}$, degassed $\mathrm{H}_{2} \mathrm{O}(1.6 \mathrm{~mL})$ was added. The reaction was allowed to stir at room temperature for $4 \mathrm{~h}$. The reaction mixture was then diluted with $\mathrm{CH}_{2} \mathrm{Cl}_{2}$ and washed with water $(2 \times 50 \mathrm{~mL})$. The organic phase was dried over $\mathrm{Na}_{2} \mathrm{SO}_{4}$, filtered, and concentrated under reduced pressure. The residue was purified by column chromatography $\left(\mathrm{SiO}_{2}, 10 \% \mathrm{CH}_{2} \mathrm{Cl}_{2} /\right.$ hexanes, $\left.\mathrm{R}_{\mathrm{f}}=0.2\right)$. The product was isolated as a yellow solid. (0.13 g, 57\% yield).

${ }^{1} \mathrm{H}$ NMR $\left(400 \mathrm{MHz}, \mathrm{CDCl}_{3}\right) \delta 7.68(\mathrm{~s}, 1 \mathrm{H}), 7.59(\mathrm{~d}, J=8.2 \mathrm{~Hz}, 2 \mathrm{H}), 7.52(\mathrm{~d}, J=8.2 \mathrm{~Hz}, 2 \mathrm{H}), 7.05(\mathrm{~d}, J=8.1$ $\mathrm{Hz}, 4 \mathrm{H}), 5.00(\mathrm{~m}, 1 \mathrm{H}), 1.51(\mathrm{~d}, J=6.5 \mathrm{~Hz}, 4 \mathrm{H}), 1.26(\mathrm{~s}, 40 \mathrm{H}), 0.89(\mathrm{t}, J=6.8 \mathrm{~Hz}, 6 \mathrm{H})$.

${ }^{13} \mathrm{C}\left\{{ }^{1} \mathrm{H}\right\}$ NMR $\left(101 \mathrm{MHz}, \mathrm{CDCl}_{3}\right) \delta 162.9,148.3,141.5,140.6,139.9,131.5,130.3,130.1,129.9,127.2$, $125.6,119.7,118.6,75.2,34.1,32.0,29.8,29.8,29.7,29.7,29.5,25.4,22.8,14.2$.

HRMS (APCI): calcd for $\mathrm{C}_{42} \mathrm{H}_{60} \mathrm{~N}_{6} \mathrm{O}_{2} \mathrm{~S}[\mathrm{M}+\mathrm{H}]^{+}:$712.4493; found: 712.4495 


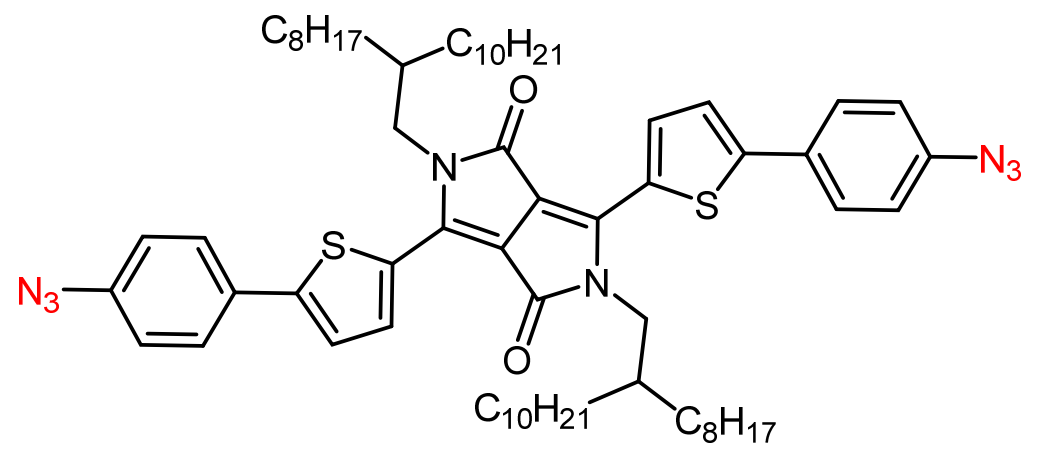

\section{3,6-bis(5-(4-azidophenyl)thiophen-2-yl)-2,5-bis(2-octyldodecyl)-2,5-dihydropyrrolo[3,4-} c]pyrrole-1,4-dione (S13). This procedure is based on a modified literature procedure. ${ }^{15}$ In an $\mathrm{N}_{2}$ filled glovebox, a Schlenk tube was charged with 3,6-bis(5-bromothiophen-2-yl)-2,5-bis(2octyldodecyl)-2,5-dihydropyrrolo[3,4-c]pyrrole-1,4-dione ${ }^{18}$ ( $0.25 \mathrm{~g}, 0.25 \mathrm{mmol}, 1.0$ equiv), 2-(4azidophenyl)-4,4,5,5-tetramethyl-1,3,2-dioxaborolane ${ }^{16}(0.13 \mathrm{~g}, 0.54 \mathrm{mmol}, 2.2$ equiv), tris(omethoxyphenyl)phosphine ( $0.042 \mathrm{~g}, 0.12 \mathrm{mmol}, 0.48$ equiv), $\mathrm{K}_{3} \mathrm{PO}_{4}(0.63 \mathrm{~g}, 2.9 \mathrm{mmol}, 12.0$ equiv.), $\mathrm{Pd}_{2}(\mathrm{dba})_{3}(0.054 \mathrm{~g}, 0.059 \mathrm{mmol}, 0.24$ equiv), Aliquat 336 (1 drop), and toluene ( $8.5 \mathrm{~mL})$. The Schlenk tube was sealed and removed from the glovebox. Under $\mathrm{N}_{2}$, degassed $\mathrm{H}_{2} \mathrm{O}(1.2 \mathrm{~mL})$ was added. The reaction was allowed to stir at room temperature for $4 \mathrm{~h}$. The reaction mixture was then diluted with $\mathrm{CH}_{2} \mathrm{Cl}_{2}$ and washed with water $(2 \times 50 \mathrm{~mL})$. The organic phase was dried over $\mathrm{Na}_{2} \mathrm{SO}_{4}$, filtered, and concentrated under reduced pressure. The residue was purified by column chromatography $\left(\mathrm{SiO}_{2}\right.$, $30 \% \mathrm{CH}_{2} \mathrm{Cl}_{2} /$ hexanes, $\left.\mathrm{R}_{\mathrm{f}}=0.1\right)$. The product was isolated as a deep blue oil. ( $0.15 \mathrm{~g}, 54 \%$ yield $)$.

${ }^{1} \mathrm{H} \mathrm{NMR}\left(300 \mathrm{MHz}, \mathrm{CDCl}_{3}\right) \delta 8.92(\mathrm{~d}, \mathrm{~J}=4.1 \mathrm{~Hz}, 2 \mathrm{H}), 7.72-7.61(\mathrm{~m}, 4 \mathrm{H}), 7.42(\mathrm{~d}, \mathrm{~J}=4.1 \mathrm{~Hz}, 2 \mathrm{H}), 7.08(\mathrm{~s}$, $4 \mathrm{H}), 4.06(\mathrm{~d}, \mathrm{~J}=7.7 \mathrm{~Hz}, 4 \mathrm{H}), 1.97(\mathrm{~s}, 2 \mathrm{H}), 1.28(\mathrm{~m}, 64 \mathrm{H}), 0.85(\mathrm{~s}, 12 \mathrm{H})$.

${ }^{13} \mathrm{C}\left\{{ }^{1} \mathrm{H}\right\}$ NMR $\left(201 \mathrm{MHz}, \mathrm{CDCl}_{3}\right) \delta 161.6,148.4,140.4,139.6,136.7,130.0,128.7,127.4,124.2,119.6$, $108.2,46.2,37.9,31.9,31.8,31.3,30.0,29.6,29.6,29.5,29.3,29.3,26.3,22.6,22.6,14.0$.

HRMS (APCI): calcd for $\mathrm{C}_{66} \mathrm{H}_{94} \mathrm{~N}_{8} \mathrm{O}_{2} \mathrm{~S}_{2}[\mathrm{M}+\mathrm{H}]^{+}:$1095.7014; found: 1095.7001 


\section{Reaction Optimization Studies}

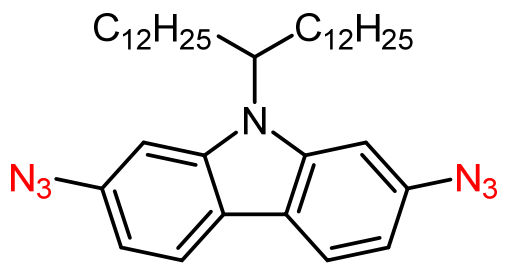

2

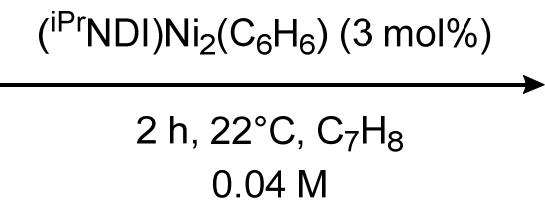

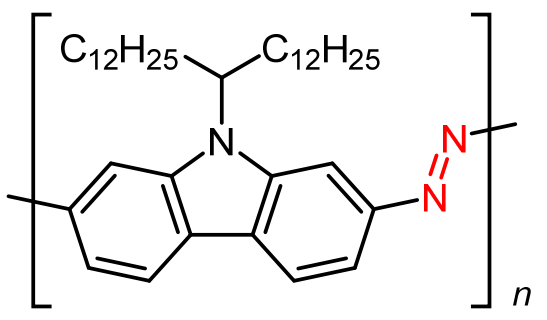

3

Standard procedure. This procedure is based on a modified literature procedure. ${ }^{9}$ In an $\mathrm{N}_{2}$ filled glovebox, a 2-dram vial equipped with a stir bar was charged with 2 (10.0 $\mathrm{mg}, 0.017 \mathrm{mmol}, 1.0$ equiv) and toluene $(0.30 \mathrm{~mL})$. Catalyst $1(0.36 \mathrm{mg}, 0.50 \mu \mathrm{mol}, 3 \mathrm{~mol} \%)$ dissolved in $\mathrm{C}_{7} \mathrm{H}_{8}(0.12 \mathrm{~mL})$ was added to the stirring diazide solution. The reaction was allowed to stir for $2 \mathrm{~h}$ at room temperature. The reaction was quenched by exposure to air. $\mathrm{MeOH}(1.0 \mathrm{~mL})$ and $\mathrm{CH}_{2} \mathrm{Cl}_{2}(1.0 \mathrm{~mL})$ were added sequentially. The resulting suspension was sonicated and gravity filtered. The red solid was washed sequentially with $\mathrm{Et}_{2} \mathrm{O}(20.0 \mathrm{~mL})$ and $\mathrm{CH}_{2} \mathrm{Cl}_{2}(20.0 \mathrm{~mL})$ to remove any low molecular weight material. The solid was allowed to air dry then collected and dried further under vacuum. Monomer conversions for entries 3 and 5 were determined by ${ }^{1} \mathrm{H}$ NMR. For entry 8 the polymer was purified using a Soxhlet apparatus. The product was washed sequentially with $\mathrm{MeOH}$, hexanes, and $\mathrm{CHCl}_{3}$ then extracted with $\mathrm{C}_{6} \mathrm{H}_{5} \mathrm{Cl}$.

\begin{tabular}{|c|c|c|c|c|c|c|}
\hline entry & deviation from Standard Conditions & $\begin{array}{c}\text { conversion } \\
\text { of } \mathbf{2}\end{array}$ & $\begin{array}{c}\mathrm{M}_{\mathrm{n}} \\
(\mathrm{kg} / \mathrm{mol})\end{array}$ & $\begin{array}{c}\mathrm{M}_{\mathrm{w}} \\
(\mathrm{kg} / \mathrm{mol})\end{array}$ & $\bigoplus_{\mathrm{M}}$ & $X_{\mathrm{n}}$ \\
\hline 1 & none & $>99 \%$ & 20.5 & 58.7 & 2.9 & 37.7 \\
\hline 2 & purification by Soxhlet extraction $\left(\mathrm{C}_{6} \mathrm{H}_{5} \mathrm{Cl}\right)$ & $>99 \%$ & 42.1 & 92.5 & 2.2 & 77.6 \\
\hline 3 & $1 \mathrm{~mol} \%$ of $\mathbf{1}$ & $31 \%$ & - & - & - & - \\
\hline 4 & $5 \mathrm{~mol} \%$ of $\mathbf{1}$ & $>99 \%$ & 28.2 & 98.5 & 3.5 & 51.8 \\
\hline 5 & {$[\mathbf{2}]=0.02 \mathrm{M}$} & $79 \%$ & - & - & - & - \\
\hline 6 & {$[\mathbf{2}]=0.08 \mathrm{M}$} & $>99 \%$ & 35.2 & 104.2 & 3.0 & 64.7 \\
\hline 7 & $60{ }^{\circ} \mathrm{C}$ & $>99 \%$ & 69.0 & 295.9 & 4.3 & 126.9 \\
\hline 8 & $\mathrm{THF}$ instead of toluene & 8.6 & 18.6 & 2.1 & 15.8 \\
\hline
\end{tabular}




\section{Azopolymer Synthesis and Characterization}

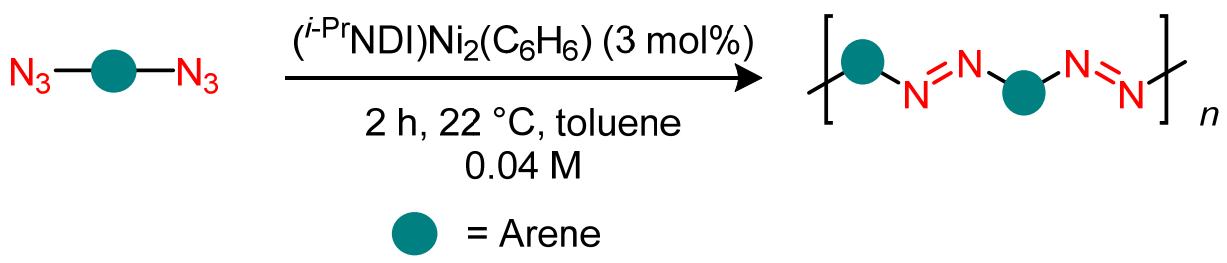

General procedure. In an $\mathrm{N}_{2}$ filled glovebox, a microwave vial was charged with a solution of the diazide (1.0 equiv) dissolved in toluene and a magnetic stir bar. A solution of catalyst 1 (3 mol\%) dissolved in toluene was added to the stirring diazide solution ( $0.04 \mathrm{M}$ final concentration of the diazide). The vial was sealed, and the reaction mixture was stirred at room temperature. After $2 \mathrm{~h}$, the reaction vial was opened to airm and methanol was added to precipitate the product. After sonication, the crude mixture was added to a thimble and purified in a Soxhlet apparatus (see below for wash and extraction solvents). Isolated polymers were obtained after drying under vacuum.

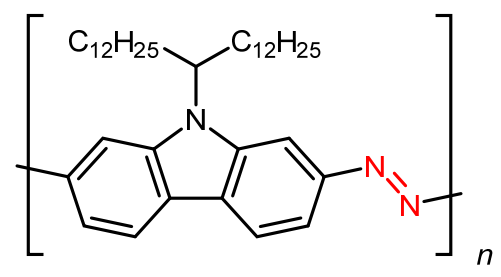

PolyAzoCarbazole (3). ${ }^{19}$ Synthesized according to the general procedure using $2(0.10 \mathrm{~g}, 0.17$ mmol, 1.0 equiv), toluene ( $4.2 \mathrm{~mL})$, and $1(3.6 \mathrm{mg}, 5.0 \mu \mathrm{mol}, 3 \mathrm{~mol} \%)$. Soxhlet wash solvents: $\mathrm{MeOH}$, hexanes, then $\mathrm{CHCl}_{3}$. Extraction solvent: $\mathrm{C}_{6} \mathrm{H}_{5} \mathrm{Cl}$. PolyAzoCarbazole (3) was isolated as a red solid. (0.061 g, 61\% yield).

${ }^{1} \mathrm{H}$ NMR $\left(400 \mathrm{MHz}, 100{ }^{\circ} \mathrm{C}\right.$, toluene- $\left.\mathrm{d}_{8}\right) \delta 8.47(\mathrm{~s}, 2 \mathrm{H}), 8.16(\mathrm{~d}, J=8.4 \mathrm{~Hz}, 2 \mathrm{H}), 8.09(\mathrm{~d}, J=8.3 \mathrm{~Hz}, 2 \mathrm{H})$, $4.70(\mathrm{~s}, 1 \mathrm{H}), 2.52-2.36(\mathrm{~m}, 2 \mathrm{H}), 1.97-1.83(\mathrm{~m}, 2 \mathrm{H}), 1.32-1.09(\mathrm{~m}, 40 \mathrm{H}), 0.86(\mathrm{t}, 6 \mathrm{H})$.

UV-Vis-NIR ( $\left.\mathrm{C}_{6} \mathrm{H}_{5} \mathrm{Cl}, 0.0326 \mathrm{mM}\right): \lambda_{\max }, \mathrm{nm}\left(\varepsilon, \mathrm{M}^{-1} \mathrm{~cm}^{-1}\right): 440(\mathrm{sh}), 470(\mathrm{sh}), 503(15,000), 538$ $(17,000)$.

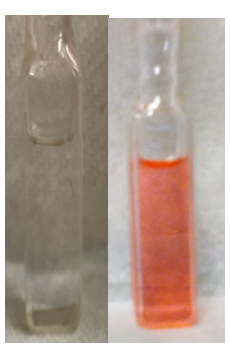

Figure S1. Photo of 2 (left) and 3 (right) in $\mathrm{C}_{6} \mathrm{H}_{5} \mathrm{Cl}$. 


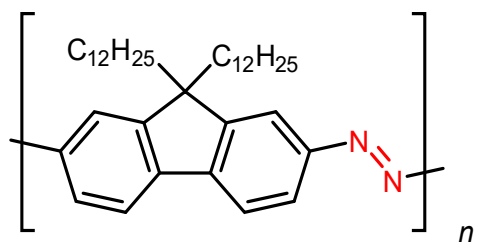

PolyAzoFluorene (6). Synthesized according to the general procedure using 2,7-diazido-9,9didodecyl-9H-fluorene ${ }^{9}(0.10 \mathrm{~g}, 0.17 \mathrm{mmol}, 1.0$ equiv), toluene $(4.3 \mathrm{~mL})$, and 1 ( $3.7 \mathrm{mg}, 5.1 \mu \mathrm{mol}, 3$ mol\%). Soxhlet wash solvents: $\mathrm{MeOH}$ then hexanes. Extraction solvent: $\mathrm{CHCl}_{3}$. PolyAzoFluorene (6) was isolated as an orange solid. ( $0.082 \mathrm{~g}$, 82\% yield). Spectral match those previously reported. ${ }^{9}$

UV-Vis-NIR ( $\left.{ }_{6} \mathrm{H}_{5} \mathrm{Cl}, 0.0566 \mathrm{mM}\right): \lambda_{\max }, \mathrm{nm}\left(\varepsilon, \mathrm{M}^{-1} \mathrm{~cm}^{-1}\right): 336(1,700) 450(\mathrm{sh}), 484(17,000) 520$ $(18,000)$

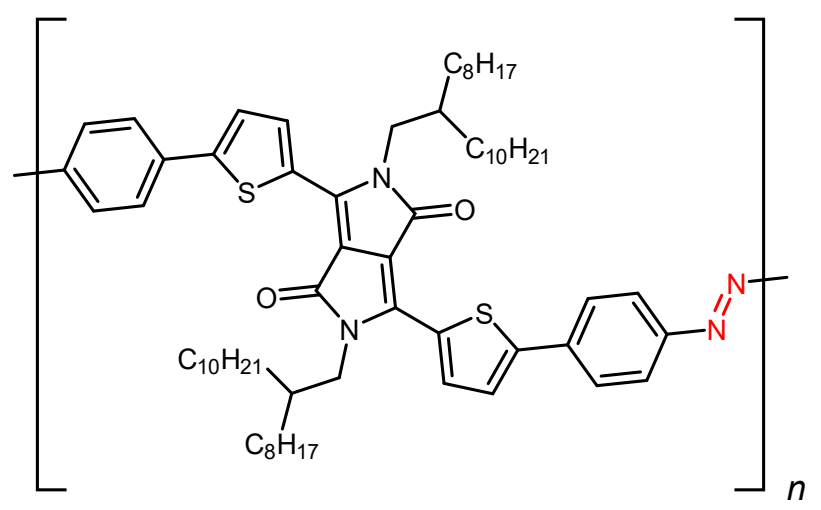

PolyAzoTDPP (7). Synthesized according to the general procedure using S13 $(0.10 \mathrm{~g}, 0.091 \mathrm{mmol}$, 1.0 equiv), toluene $(2.3 \mathrm{~mL})$, and $\mathbf{1}(2.0 \mathrm{mg}, 2.7 \mu \mathrm{mol}, 3 \mathrm{~mol} \%)$. Soxhlet wash solvents: $\mathrm{MeOH}$ then hexanes. Extraction solvent: $\mathrm{CHCl}_{3}$. PolyAzoTDPP (7) was isolated as a green solid. (0.077 g, 77\% yield).

${ }^{1} \mathrm{H}$ NMR (400 MHz, toluene- $\left.\mathrm{d}_{8}\right) \delta 9.44-9.15(\mathrm{~m}, 2 \mathrm{H}), 7.93(\mathrm{~d}, J=8.1 \mathrm{~Hz}, 3 \mathrm{H}), 7.62(\mathrm{~d}, J=8.4 \mathrm{~Hz}, 3 \mathrm{H})$, $4.20(\mathrm{~s}, 4 \mathrm{H}), 2.21(\mathrm{~s}, 2 \mathrm{H}), 1.60-1.22(\mathrm{~m}, 64 \mathrm{H}), 0.93-0.86(\mathrm{~m}, 12 \mathrm{H})$.

UV-Vis-NIR ( $\left.\mathrm{C}_{6} \mathrm{H}_{5} \mathrm{Cl}, 0.0385 \mathrm{mM}\right): \lambda_{\max }, \mathrm{nm}\left(\varepsilon, \mathrm{M}^{-1} \mathrm{~cm}^{-1}\right): 334(1,300) 426(3,500), 664(8,600), 746$ $(\mathrm{sh})$

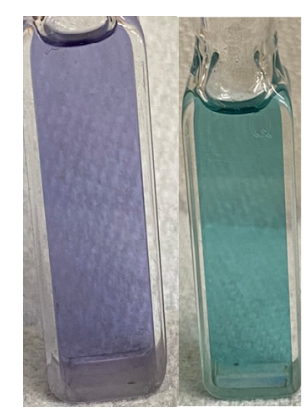

Figure S2. Photo of $\mathbf{S 1 3}$ (left) and $\mathbf{7}$ (right) in $\mathrm{C}_{6} \mathrm{H}_{5} \mathrm{Cl}$. 
PolyAzoThiophene (8). Synthesized according to the general procedure using S12 $(0.10 \mathrm{~g}, 0.14$ mmol, 1.0 equiv), toluene ( $3.5 \mathrm{~mL}$ ), and $\mathbf{1}$ ( $3.1 \mathrm{mg}, 4.2 \mu \mathrm{mol}, 3 \mathrm{~mol} \%)$. Soxhlet wash solvents: $\mathrm{MeOH}$ then hexanes. Extraction solvent: $\mathrm{CHCl}_{3}$. PolyAzoThiophene (8) was isolated as an orange solid. (0.081 g, $81 \%$ yield).

${ }^{1} \mathrm{H}$ NMR (400 MHz, $100{ }^{\circ} \mathrm{C}$, toluene- $\left.\mathrm{d}_{8}\right) \delta 8.05(\mathrm{~s}, 2 \mathrm{H}), 7.97(\mathrm{~s}, 3 \mathrm{H}), 7.72(\mathrm{~s}, 2 \mathrm{H}), 7.58(\mathrm{~s}, 2 \mathrm{H}), 5.23(\mathrm{~s}$, $1 \mathrm{H}), 1.68-1.44(\mathrm{~m}, 4 \mathrm{H}), 1.31(\mathrm{~s}, 40 \mathrm{H}), 0.90(\mathrm{~s}, 6 \mathrm{H})$.

UV-Vis-NIR $\left(\mathrm{C}_{6} \mathrm{H}_{5} \mathrm{Cl}, 0.0135 \mathrm{mM}\right): \lambda_{\max }, \mathrm{nm}\left(\varepsilon, \mathrm{M}^{-1} \mathrm{~cm}^{-1}\right): 427(15,000)$

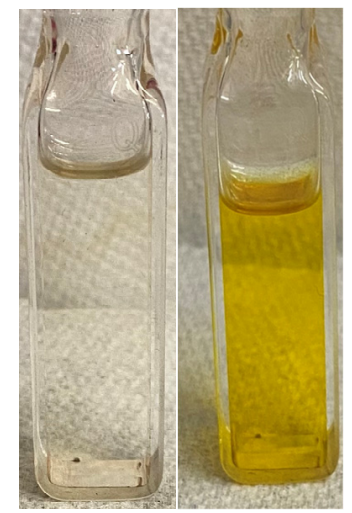

Figure S3. Photo of $\mathbf{S 1 2}$ (left) and $\mathbf{8}$ (right) in $\mathrm{C}_{6} \mathrm{H}_{5} \mathrm{Cl}$. 


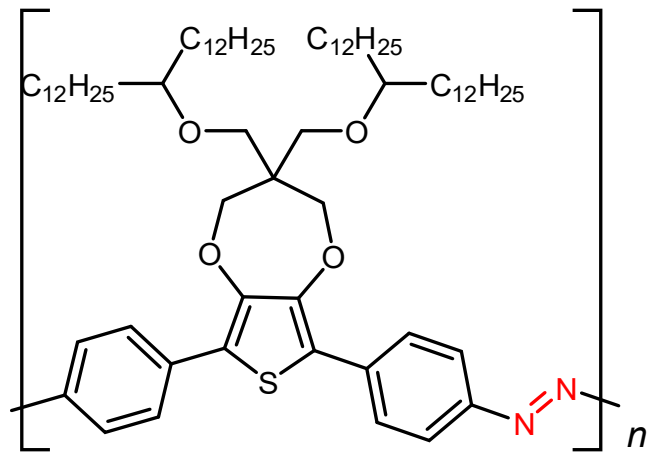

PolyAzoProD0T (9). Synthesized according to the general procedure using $\mathbf{S 1 0}(0.10 \mathrm{~g}, 0.087$ mmol, 1.0 equiv), toluene ( $2.2 \mathrm{~mL})$, and $1(1.9 \mathrm{mg}, 2.6 \mu \mathrm{mol}, 3 \mathrm{~mol} \%)$. Soxhlet wash solvent: $\mathrm{MeOH}$. Extraction solvent: hexanes. PolyAzoProDOT (9) was isolated as a red solid. (0.097 g, 97\% yield).

${ }^{1} \mathrm{H} \mathrm{NMR}\left(400 \mathrm{MHz}, 50{ }^{\circ} \mathrm{C}, \mathrm{CDCl}_{3}\right) \delta 8.04-7.86(\mathrm{~m}, 8 \mathrm{H}), 4.27(\mathrm{~s}, 4 \mathrm{H}), 3.65(\mathrm{~s}, 4 \mathrm{H}), 3.31-3.23(\mathrm{~m}, 2 \mathrm{H})$, $1.57-1.45(\mathrm{~m}, 8 \mathrm{H}), 1.37-1.23(\mathrm{~m}, 80 \mathrm{H}), 0.88(\mathrm{t}, 12 \mathrm{H})$.

UV-Vis-NIR ( $\left.\mathrm{C}_{6} \mathrm{H}_{5} \mathrm{Cl}, 0.0456 \mathrm{mM}\right): \lambda_{\max }, \mathrm{nm}\left(\varepsilon, \mathrm{M}^{-1} \mathrm{~cm}^{-1}\right): 514$ (20,000), $543(\mathrm{sh})$

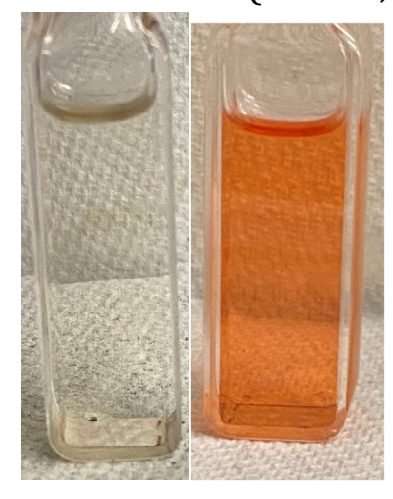

Figure S4. Photo of $\mathbf{S 1 0}$ (left) and $\mathbf{9}$ (right) in $\mathrm{C}_{6} \mathrm{H}_{5} \mathrm{Cl}$. 


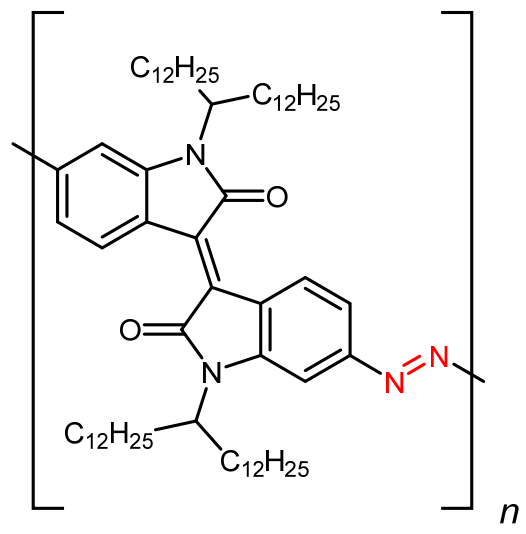

PolyAzoIsoindigo (10). Synthesized according to the general procedure using S7 (0.098 g, 0.094 mmol, 1.0 equiv), toluene ( $2.3 \mathrm{~mL}$ ), and $\mathbf{1}$ ( $6.8 \mathrm{mg}, 9.4 \mu \mathrm{mol}, 10 \mathrm{~mol} \%)$. Soxhlet wash solvents: $\mathrm{MeOH}$ then hexanes. Extraction solvent: $\mathrm{CHCl}_{3}$. PolyAzoIsoindigo (10) was isolated as a green solid. $(0.091 \mathrm{~g}$, $93 \%$ yield).

${ }^{1} \mathrm{H}$ NMR (400 MHz, $100{ }^{\circ} \mathrm{C}$, toluene-ds) $\delta 9.81(\mathrm{~s}, 2 \mathrm{H}), 7.91-7.50(\mathrm{~m}, 4 \mathrm{H}), 4.52(\mathrm{~s}, 2 \mathrm{H}), 2.19(\mathrm{~s}, 4 \mathrm{H})$, $1.77(\mathrm{~s}, 4 \mathrm{H}), 1.31(\mathrm{~s}, 80 \mathrm{H}), 0.89(\mathrm{~s}, 12 \mathrm{H})$.

UV-Vis-NIR ( $\left.\mathrm{C}_{6} \mathrm{H}_{5} \mathrm{Cl}, 0.0606 \mathrm{mM}\right): \lambda_{\max }, \mathrm{nm}\left(\varepsilon, \mathrm{M}^{-1} \mathrm{~cm}^{-1}\right): 381$ (sh), 481 (17,000), $664(25,000), 709$ $(26,000)$.

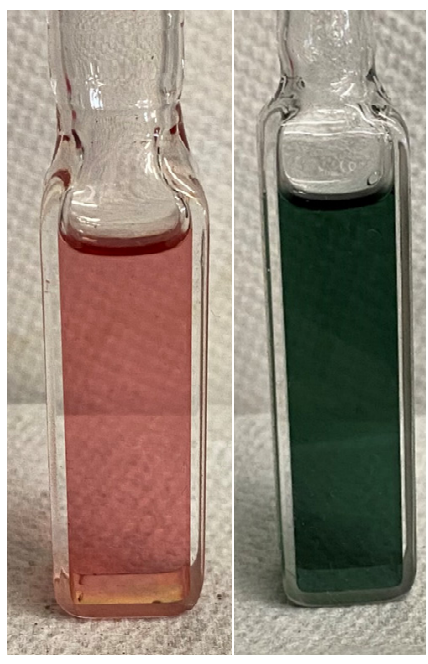

Figure S5. Photo of $\mathbf{S 7}$ (left) and $\mathbf{1 0}$ (right) in $\mathrm{C}_{6} \mathrm{H}_{5} \mathrm{Cl}$. 


\section{Copolymerization Experiments}

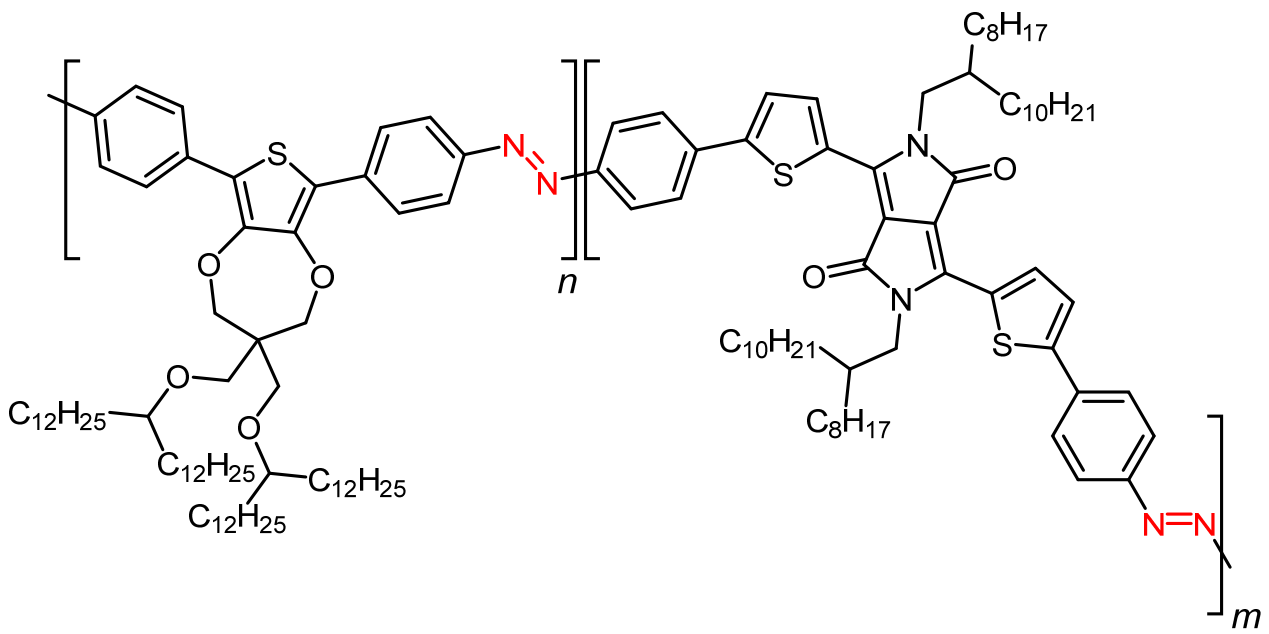

ProDOT/TDPP copolymerization at 1:1 (11). In an $\mathrm{N}_{2}$ filled glovebox, a 2-dram vial was charged with a magnetic stir bar, $\mathbf{S 1 0}(0.010 \mathrm{~g}, 8.7 \mu \mathrm{mol}, 1.0$ equiv), $\mathbf{S 1 3}(0.010 \mathrm{~g}, 9.1 \mu \mathrm{mol}, 1.0$ equiv) and toluene $(0.22 \mathrm{~mL})$. Catalyst 1 ( $3.9 \mathrm{mg}, 0.55 \mu \mathrm{mol}, 3 \mathrm{~mol} \%$ total) dissolved in toluene $(0.22 \mathrm{~mL})$ was added to the stirring solution of the diazides. The vial was sealed, and the reaction mixture was stirred at room temperature. After $2 \mathrm{~h}$, the reaction vial was opened to air, and $\mathrm{MeOH}$ was added to precipitate the product. After sonication, the crude material was filtered, and the precipitate was washed with $\mathrm{Et}_{2} \mathrm{O}(10.0 \mathrm{~mL})$ and $\mathrm{CH}_{2} \mathrm{Cl}_{2}(10.0 \mathrm{~mL})$. The solid was allowed to air dry then collected and dried further under vacuum to yield the product as a dark purple solid. (0.021 g, >99\% yield). Ratio of ProDOT (9) to TDPP (7) by ${ }^{1} \mathrm{H}$ NMR integration (1:1.04).

UV-Vis-NIR ( $\left.\mathrm{C}_{6} \mathrm{H}_{5} \mathrm{Cl}, 0.0187 \mathrm{mM}\right): \lambda_{\max }, \mathrm{nm}\left(\varepsilon, \mathrm{M}^{-1} \mathrm{~cm}^{-1}\right): 327$ (sh), 442 (sh), $534(7,900), 659$ (sh), 710 $(13,000)$

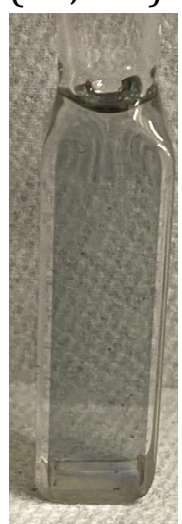

Figure S5. Photo of 11 in $\mathrm{C}_{6} \mathrm{H}_{5} \mathrm{Cl}$. 
ProDOT/TDPP copolymerization at 3:1 (12). In an $\mathrm{N}_{2}$ filled glovebox, a 2-dram vial was charged with a magnetic stir bar, $\mathbf{S 1 0}(0.015 \mathrm{~g}, 13.0 \mu \mathrm{mol}, 1.0$ equiv), $\mathbf{S 1 3}(5.0 \mathrm{mg}, 4.6 \mu \mathrm{mol}, 0.35$ equiv) and toluene $(0.22 \mathrm{~mL})$. Catalyst 1 ( $3.9 \mathrm{mg}, 0.55 \mu \mathrm{mol}, 3 \mathrm{~mol} \%$ total) dissolved in toluene $(0.22 \mathrm{~mL})$ was added to the stirring solution of the diazides. The vial was sealed, and the reaction mixture was stirred at room temperature. After $2 \mathrm{~h}$, the reaction vial was opened to air, and $\mathrm{MeOH}$ was added to precipitate the product. After sonication, the crude material was filtered, and the precipitate was washed with $\mathrm{Et}_{2} \mathrm{O}(10.0 \mathrm{~mL})$ and $\mathrm{CH}_{2} \mathrm{Cl}_{2}(10.0 \mathrm{~mL})$. The solid was allowed to air dry then collected and dried further under vacuum to yield the product as a purple solid. ( $0.021 \mathrm{~g}$, $>99 \%$ yield). Ratio of ProDOT (9) to TDPP (7) by ${ }^{1} \mathrm{H}$ NMR integration (2.6:1).

UV-Vis-NIR ( $\left.\mathrm{C}_{6} \mathrm{H}_{5} \mathrm{Cl}, 0.0351 \mathrm{mM}\right): \lambda_{\max }, \mathrm{nm}\left(\varepsilon, \mathrm{M}^{-1} \mathrm{~cm}^{-1}\right): 521(11,000), 644(\mathrm{sh}), 692(6,300)$

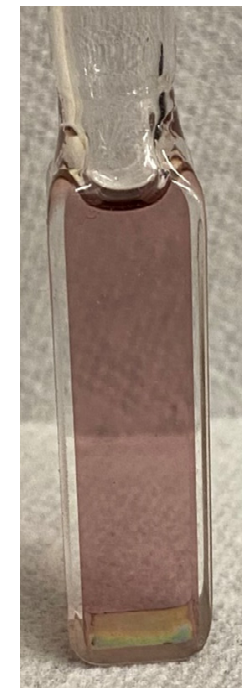

Figure S6. Photo of 12 in $\mathrm{C}_{6} \mathrm{H}_{5} \mathrm{Cl}$. 


\section{Endcapping Experiments}

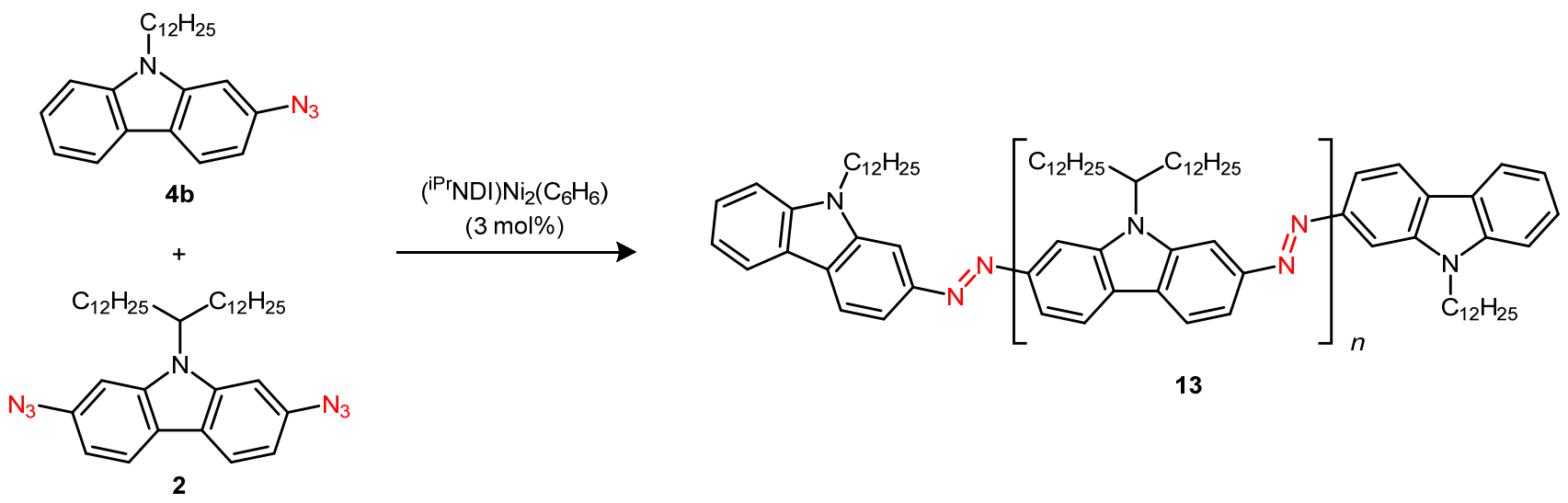

Carbazole end group incorporation (13). In an $\mathrm{N}_{2}$ filled glovebox, a 2-dram vial was charged with a magnetic stir bar, $2(0.015 \mathrm{~g}, 0.025 \mathrm{mmol}, 1.0$ equiv), $4 \mathbf{b}(0.50 \mathrm{mg}, 1.3 \mu \mathrm{mol}, 0.050$ equiv) and toluene $(0.50 \mathrm{~mL})$. Catalyst $1(0.60 \mathrm{mg}, 0.69 \mu \mathrm{mol}, 3 \mathrm{~mol} \%)$ dissolved in toluene $(0.13 \mathrm{~mL})$ was added to the stirring solution of the azides. The vial was sealed, and the reaction mixture was stirred at room temperature. After $2 \mathrm{~h}$, the reaction vial was opened to air, and $\mathrm{MeOH}(3.0 \mathrm{~mL})$ was added to precipitate the product. After sonication, the crude material was filtered, and the precipitate was washed with $\mathrm{Et}_{2} \mathrm{O}(10.0 \mathrm{~mL})$ and $\mathrm{CH}_{2} \mathrm{Cl}_{2}(10.0 \mathrm{~mL})$. The solid was allowed to air dry then collected and dried further under vacuum to yield the product as a red solid ( $0.010 \mathrm{~g}, 67 \%$ yield). Ratio of repeat units to end groups by ${ }^{1} \mathrm{H}$ NMR integration (17:1).

${ }^{1} \mathrm{H}$ NMR of 13 end group $\left(400 \mathrm{MHz}, 100{ }^{\circ} \mathrm{C}\right.$, toluene- $\left.d 8\right) \delta 7.97(\mathrm{~d}, J=7.9 \mathrm{~Hz}, 1 \mathrm{H}), 4.02(\mathrm{br} \mathrm{s}, 2 \mathrm{H})$.

${ }^{1} \mathrm{H}$ NMR of 13 repeat unit $\left(400 \mathrm{MHz}, 100^{\circ} \mathrm{C}\right.$, toluene- $\left.\mathrm{d}_{8}\right) \delta 8.47(\mathrm{~s}, 2 \mathrm{H}), 8.16(\mathrm{~d}, J=8.7 \mathrm{~Hz}, 2 \mathrm{H}), 8.09(\mathrm{~d}, J$ $=8.8 \mathrm{~Hz}, 2 \mathrm{H}), 4.70(\mathrm{~s}, 1 \mathrm{H}), 2.42(\mathrm{~s}, 2 \mathrm{H}), 1.89(\mathrm{~s}, 2 \mathrm{H}), 1.19(\mathrm{~d}, J=33.8 \mathrm{~Hz}, 40 \mathrm{H}), 0.86(\mathrm{~d}, J=7.2 \mathrm{~Hz}, 6 \mathrm{H})$.

UV-Vis-NIR ( $\left.\mathrm{C}_{6} \mathrm{H}_{5} \mathrm{Cl}, 0.0428 \mathrm{mM}\right): \lambda_{\max }, \mathrm{nm}\left(\varepsilon, \mathrm{M}^{-1} \mathrm{~cm}^{-1}\right): 435$ (sh), 465 (sh), 499 (21,000), 532 $(22,000)$. 

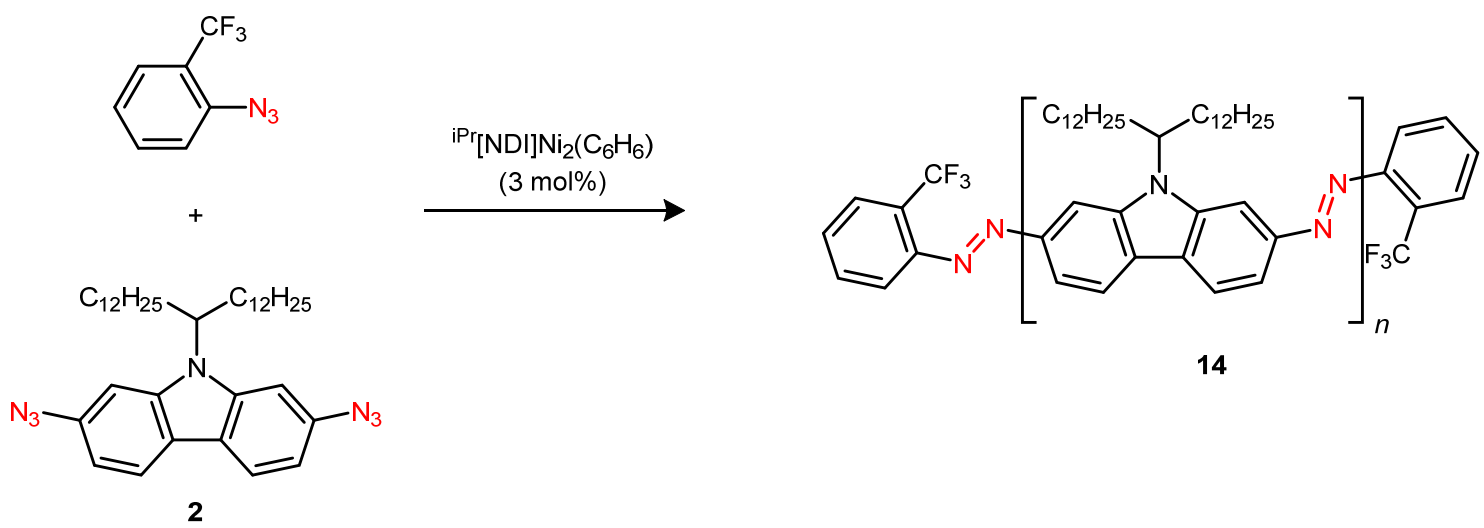

2-(trifluoromethyl)phenyl end group incorporation (14). In an $\mathrm{N}_{2}$ filled glovebox, a 2-dram vial was charged with a magnetic stir bar, $2(0.015 \mathrm{~g}, 0.025 \mathrm{mmol}, 1.0$ equiv), 2-(trifluoromethyl)phenyl azide $^{9}(0.20 \mathrm{mg}, 1.3 \mu \mathrm{mol}, 0.050$ equiv) and toluene $(0.50 \mathrm{~mL})$. Catalyst $1(0.60 \mathrm{mg}, 0.69 \mu \mathrm{mol}, 3 \mathrm{~mol}$ $\%)$ dissolved in toluene $(0.13 \mathrm{~mL})$ was added to the stirring solution of the azides. The vial was sealed, and the reaction mixture was stirred at room temperature. After $2 \mathrm{~h}$, the reaction vial was opened to air, and $\mathrm{MeOH}(3.0 \mathrm{~mL})$ was added to precipitate the product. After sonication, the crude material was filtered, and the precipitate was washed with $\mathrm{Et}_{2} \mathrm{O}(10.0 \mathrm{~mL})$ and $\mathrm{CH}_{2} \mathrm{Cl}_{2}(10.0 \mathrm{~mL})$. The solid was allowed to air dry then collected and dried further under vacuum to yield the product as a red solid (8.9 mg, 60\% yield). Ratio of repeat units to end groups by ${ }^{1} \mathrm{H}$ NMR integration (30:1).

${ }^{1} \mathrm{H}$ NMR of 14 end group $\left(400 \mathrm{MHz}, 100{ }^{\circ} \mathrm{C}\right.$, toluene- $\left.d_{8}\right) \delta 7.79(\mathrm{~d}, J=8.2 \mathrm{~Hz}, 1 \mathrm{H}), 7.58(\mathrm{~d}, J=7.7 \mathrm{~Hz}, 1 \mathrm{H})$

${ }^{1} \mathrm{H}$ NMR of 14 repeat unit $\left(400 \mathrm{MHz}, 100{ }^{\circ} \mathrm{C}\right.$, toluene- $\left.\mathrm{d}_{8}\right) \delta 8.47(\mathrm{~s}, 2 \mathrm{H}), 8.16(\mathrm{~d}, J=8.7 \mathrm{~Hz}, 2 \mathrm{H}), 8.09(\mathrm{~d}, J$ $=8.8 \mathrm{~Hz}, 2 \mathrm{H}), 4.70(\mathrm{~s}, 1 \mathrm{H}), 2.42(\mathrm{~s}, 2 \mathrm{H}), 1.89(\mathrm{~s}, 2 \mathrm{H}), 1.19(\mathrm{~d}, J=33.8 \mathrm{~Hz}, 40 \mathrm{H}), 0.86(\mathrm{~d}, J=7.2 \mathrm{~Hz}, 6 \mathrm{H})$.

${ }^{19} \mathrm{~F}$ NMR of $14\left(376 \mathrm{MHz}, 100{ }^{\circ} \mathrm{C}\right.$, toluene- $\left.d_{8}\right) \delta-58.8$.

UV-Vis-NIR ( $\left.\mathrm{C}_{6} \mathrm{H}_{5} \mathrm{Cl}, 0.0428 \mathrm{mM}\right): \lambda_{\max }, \mathrm{nm}\left(\varepsilon, \mathrm{M}^{-1} \mathrm{~cm}^{-1}\right): 435$ (sh), 465 (sh), 499 (17,000), 532 $(18,000)$. 


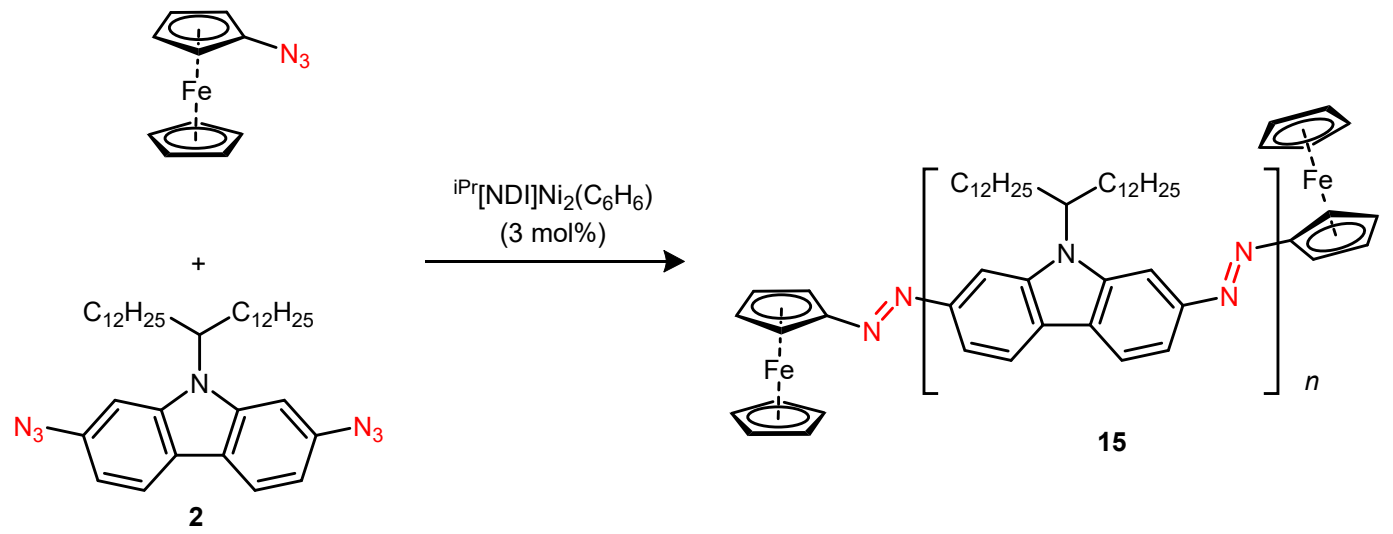

Ferrocene end group incorporation (15). In an $\mathrm{N}_{2}$ filled glovebox, a 2-dram vial was charged with a magnetic stir bar, $2\left(0.015 \mathrm{~g}, 0.025 \mathrm{mmol}, 1.0\right.$ equiv.), azidoferrocene ${ }^{9}(0.30 \mathrm{mg}, 1.3 \mu \mathrm{mol}, 0.050$ equiv.) and toluene ( $0.50 \mathrm{~mL})$. Catalyst $1(0.60 \mathrm{mg}, 0.69 \mu \mathrm{mol}, 3 \mathrm{~mol} \%)$ dissolved in toluene $(0.13 \mathrm{~mL})$ was added to the stirring solution of the azides. The vial was sealed, and the reaction mixture was stirred at room temperature. After $2 \mathrm{~h}$, the reaction vial was opened to air, and $\mathrm{MeOH}(3.0 \mathrm{~mL})$ was added to precipitate the product. After sonication, the crude material was filtered, and the precipitate was washed with $\mathrm{Et}_{2} \mathrm{O}(10.0 \mathrm{~mL})$ and $\mathrm{CH}_{2} \mathrm{Cl}_{2}(10.0 \mathrm{~mL})$. The solid was allowed to air dry then collected and dried further under vacuum to yield the product as a red solid ( $9.1 \mathrm{mg}, 60 \%$ yield). Ratio of repeat units to end groups by ${ }^{1} \mathrm{H}$ NMR integration (34:1).

${ }^{1} \mathrm{H}$ NMR of 15 end group $\left(400 \mathrm{MHz}, 100{ }^{\circ} \mathrm{C}\right.$, toluene- $\left.d_{8}\right) \delta 5.15(\mathrm{t}, J=2.0 \mathrm{~Hz}, 2 \mathrm{H}), 4.31(\mathrm{t}, J=2.0 \mathrm{~Hz}, 2 \mathrm{H})$, $4.10(\mathrm{~s}, 5 \mathrm{H})$.

${ }^{1} \mathrm{H}$ NMR of 15 repeat unit $\left(400 \mathrm{MHz}, 100{ }^{\circ} \mathrm{C}\right.$, toluene- $\left.\mathrm{d}_{8}\right) \delta 8.47(\mathrm{~s}, 2 \mathrm{H}), 8.16(\mathrm{~d}, J=8.7 \mathrm{~Hz}, 2 \mathrm{H}), 8.09(\mathrm{~d}, J$ $=8.8 \mathrm{~Hz}, 2 \mathrm{H}), 4.70(\mathrm{~s}, 1 \mathrm{H}), 2.42(\mathrm{~s}, 2 \mathrm{H}), 1.89(\mathrm{~s}, 2 \mathrm{H}), 1.19(\mathrm{~d}, J=33.8 \mathrm{~Hz}, 40 \mathrm{H}), 0.86(\mathrm{~d}, J=7.2 \mathrm{~Hz}, 6 \mathrm{H})$.

UV-Vis-NIR ( $\left.{ }_{6} \mathrm{H}_{5} \mathrm{Cl}, 0.0551 \mathrm{mM}\right): \lambda_{\max }, \mathrm{nm}\left(\varepsilon, \mathrm{M}^{-1} \mathrm{~cm}^{-1}\right): 432$ (sh), 468 (sh), $501(6,200), 533(7,200)$. 


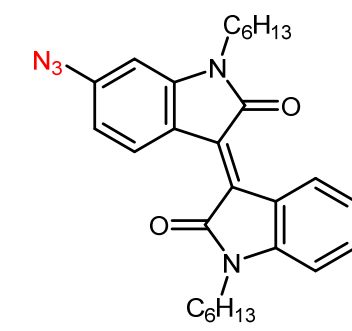

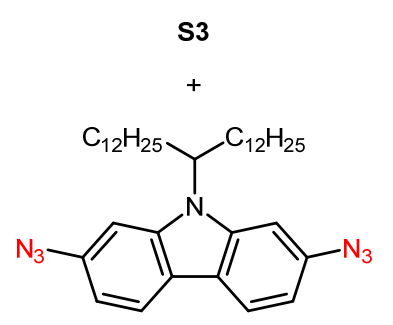

${ }^{\mathrm{iPr}}[\mathrm{NDI}] \mathrm{Ni}_{2}\left(\mathrm{C}_{6} \mathrm{H}_{6}\right)$ $(3 \mathrm{~mol} \%)$

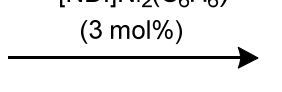

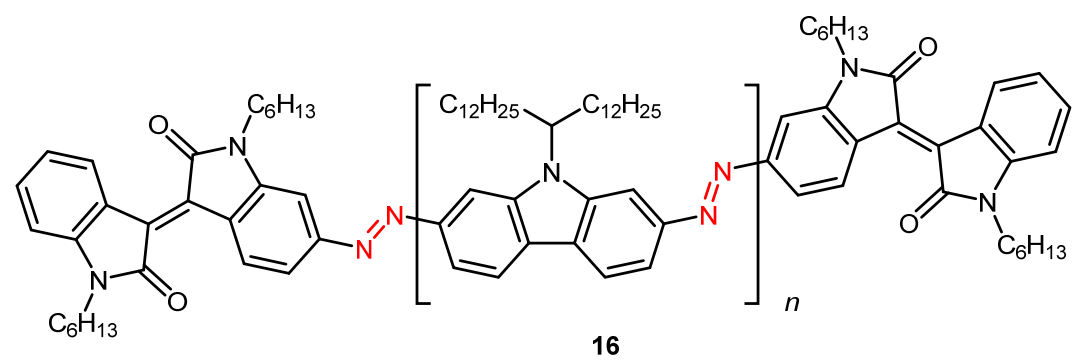

16

Isoindigo end group incorporation (16). In an $\mathrm{N}_{2}$ filled glovebox, a 2-dram vial was charged with a magnetic stir bar, 2 ( $0.015 \mathrm{~g}, 0.025 \mathrm{mmol}, 1.0$ equiv.), S3 (0.60 mg, $1.3 \mu \mathrm{mol}, 0.050$ equiv.) and toluene $(0.50 \mathrm{~mL})$. Catalyst $1(0.60 \mathrm{mg}, 0.69 \mu \mathrm{mol}, 3 \mathrm{~mol} \%)$ dissolved in toluene $(0.13 \mathrm{~mL})$ was added to the stirring solution of the azides. The vial was sealed, and the reaction mixture was stirred at room temperature. After $2 \mathrm{~h}$, the reaction vial was opened to air, and $\mathrm{MeOH}(3.0 \mathrm{~mL}$ ) was added to precipitate the product. After sonication, the crude material was filtered, and the precipitate was washed with $\mathrm{Et}_{2} \mathrm{O}(10.0 \mathrm{~mL})$ and $\mathrm{CH}_{2} \mathrm{Cl}_{2}(10.0 \mathrm{~mL})$. The solid was allowed to air dry then collected and dried further under vacuum to yield the product as a red solid (11.0 mg, 73\% yield). Ratio of repeat units to end groups by ${ }^{1} \mathrm{H}$ NMR integration (37:1).

${ }^{1} \mathrm{H}$ NMR of 16 end group (400 MHz, $100{ }^{\circ} \mathrm{C}$, toluene- $\left.d_{8}\right) \delta 9.83$ (br s, $1 \mathrm{H}$ ), 9.62 (br s, $1 \mathrm{H}$ ), 3.65 (br s, 2H).

${ }^{1} \mathrm{H}$ NMR of 16 repeat unit (400 MHz, $100{ }^{\circ} \mathrm{C}$, toluene- $\left.\mathrm{d}_{8}\right) \delta 8.47(\mathrm{~s}, 2 \mathrm{H}), 8.16(\mathrm{~d}, J=8.7 \mathrm{~Hz}, 2 \mathrm{H}), 8.09(\mathrm{~d}, J$ $=8.8 \mathrm{~Hz}, 2 \mathrm{H}), 4.70(\mathrm{~s}, 1 \mathrm{H}), 2.42(\mathrm{~s}, 2 \mathrm{H}), 1.89(\mathrm{~s}, 2 \mathrm{H}), 1.19(\mathrm{~d}, J=33.8 \mathrm{~Hz}, 40 \mathrm{H}), 0.86(\mathrm{~d}, J=7.2 \mathrm{~Hz}, 6 \mathrm{H})$.

UV-Vis-NIR ( $\left.\mathrm{C}_{6} \mathrm{H}_{5} \mathrm{Cl}, 0.0612 \mathrm{mM}\right): \lambda_{\max }, \mathrm{nm}\left(\varepsilon, \mathrm{M}^{-1} \mathrm{~cm}^{-1}\right): 432(\mathrm{sh}), 468(\mathrm{sh}), 501$ (23,000), 534 $(24,000)$ 


\section{Lewis Acid Experiments}<smiles>O=[N+]([O-])c1cccc(/N=N/c2ccc3c4ccccc4n([O-])c3c2)c1</smiles>

$5 a$

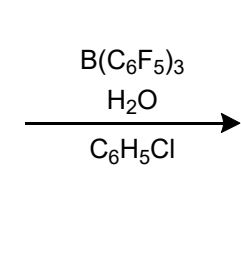

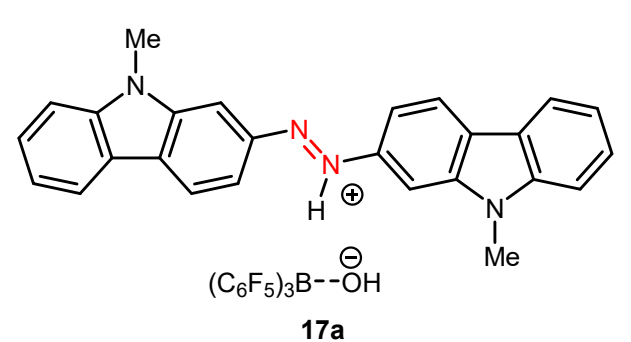

$17 a$

Azocarbazole $\mathbf{B}\left(\mathbf{C}_{6} \mathbf{F}_{5}\right)_{3} / \mathbf{H}_{2} \mathbf{O}$ adduct (17a). An NMR tube was charged with $\mathbf{5 a}(2.0 \mathrm{mg}, 5.2 \mu \mathrm{mol}, 1.0$ equiv) dissolved in $\left.\mathrm{C}_{6} \mathrm{H}_{5} \mathrm{Cl}(0.40 \mathrm{~mL}) . \mathrm{B}_{(} \mathrm{C}_{6} \mathrm{~F}_{5}\right)_{3}(10.0 \mathrm{mg}, 0.020 \mathrm{mmol}, 3.8$ equiv) was added as a solid to solution, resulting in an immediate color change to deep blue. $\mathrm{C}_{6} \mathrm{H}_{5} \mathrm{Cl}(0.10 \mathrm{~mL})$ was layered on top of the solution followed by pentane $(0.70 \mathrm{~mL})$. The solvents were allowed to mix for 7 days, and single crystals suitable for X-ray diffraction were obtained.

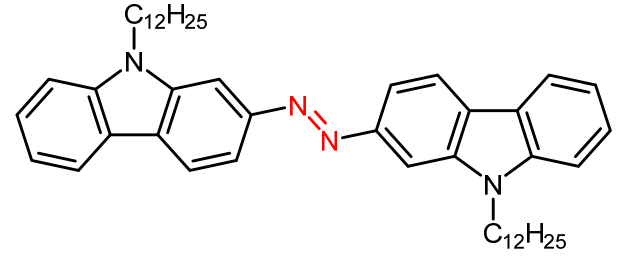

5b

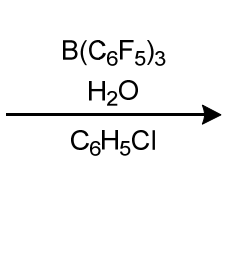

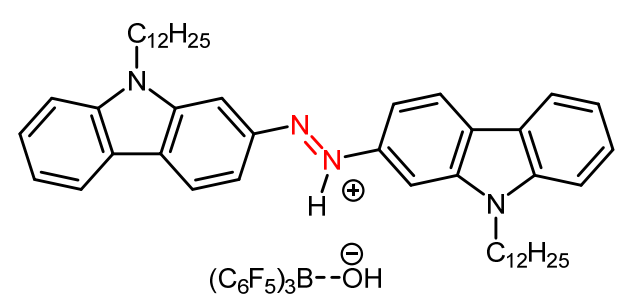

$17 b$

Azocarbazole $\mathbf{B}\left(\mathbf{C}_{6} \mathbf{F}_{5}\right)_{3} / \mathbf{H}_{2} \mathbf{O}$ adduct (17b). A vial was charged with $\mathbf{5 b}(2.0 \mathrm{mg}, 2.9 \mu \mathrm{mol}, 1.0$ equiv) and $\mathrm{C}_{6} \mathrm{H}_{5} \mathrm{Cl}(0.40 \mathrm{~mL}) . \mathrm{B}\left(\mathrm{C}_{6} \mathrm{~F}_{5}\right)_{3}(10.0 \mathrm{mg}, 0.020 \mathrm{mmol}, 3.8$ equiv) was added as a solid to solution, resulting in a color change to deep blue.

${ }^{1} \mathrm{H}$ NMR $\left(300 \mathrm{MHz}^{\mathrm{CDCl}} 3\right) \delta 8.16(\mathrm{t}, J=8.7 \mathrm{~Hz}, 4 \mathrm{H}), 8.08(\mathrm{~d}, J=1.7 \mathrm{~Hz}, 2 \mathrm{H}), 7.89(\mathrm{~d}, J=8.6,2 \mathrm{H}), 7.61(\mathrm{t}, J$ $=7.7 \mathrm{~Hz}, 2 \mathrm{H}), 7.46(\mathrm{~d}, J=8.3 \mathrm{~Hz}, 2 \mathrm{H}), 7.33(\mathrm{t}, J=7.5 \mathrm{~Hz}, 2 \mathrm{H}), 4.34(\mathrm{t}, J=7.2 \mathrm{~Hz}, 4 \mathrm{H}), 1.91(\mathrm{t}, J=7.3 \mathrm{~Hz}$, $4 \mathrm{H}), 1.35(\mathrm{~s}, 8 \mathrm{H}), 1.24(\mathrm{~d}, J=8.1 \mathrm{~Hz}, 28 \mathrm{H}), 0.85(\mathrm{t}, J=6.6 \mathrm{~Hz}, 6 \mathrm{H})$.

${ }^{19} \mathrm{~F}$ NMR $\left(282 \mathrm{MHz}, \mathrm{CDCl}_{3}\right) \delta-136.98(\mathrm{~d}),-159.12(\mathrm{t}, \mathrm{J}=20.3 \mathrm{~Hz}),-165.28(\mathrm{t})$.

UV-Vis-NIR ( $\left.{ }_{6} \mathrm{H}_{5} \mathrm{Cl}, 0.143 \mathrm{mM}\right): \lambda_{\max }, \mathrm{nm}\left(\varepsilon, \mathrm{M}^{-1} \mathrm{~cm}^{-1}\right): 316(3,200), 626(11,000)$
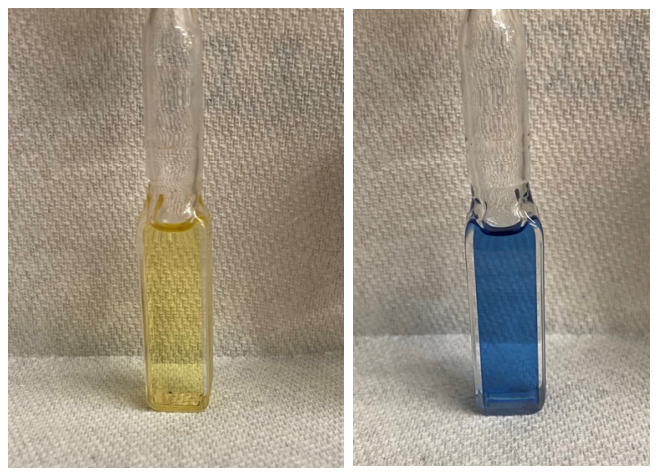

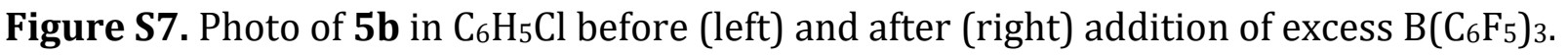




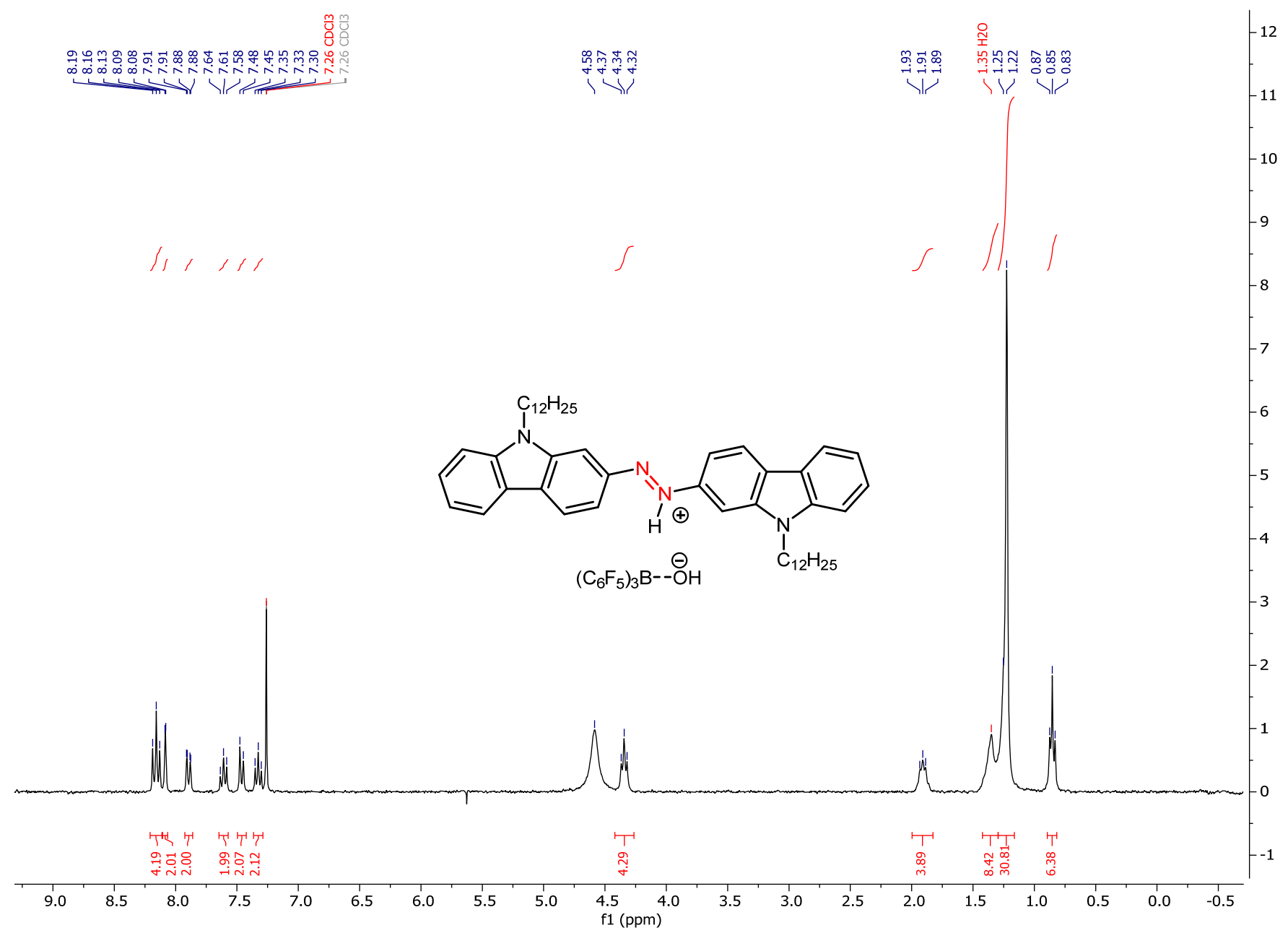

Figure S8. ${ }^{1} \mathrm{H}$ NMR spectrum of $\mathbf{1 7 b}\left(300 \mathrm{MHz}, \mathrm{CDCl}_{3}\right)$. 


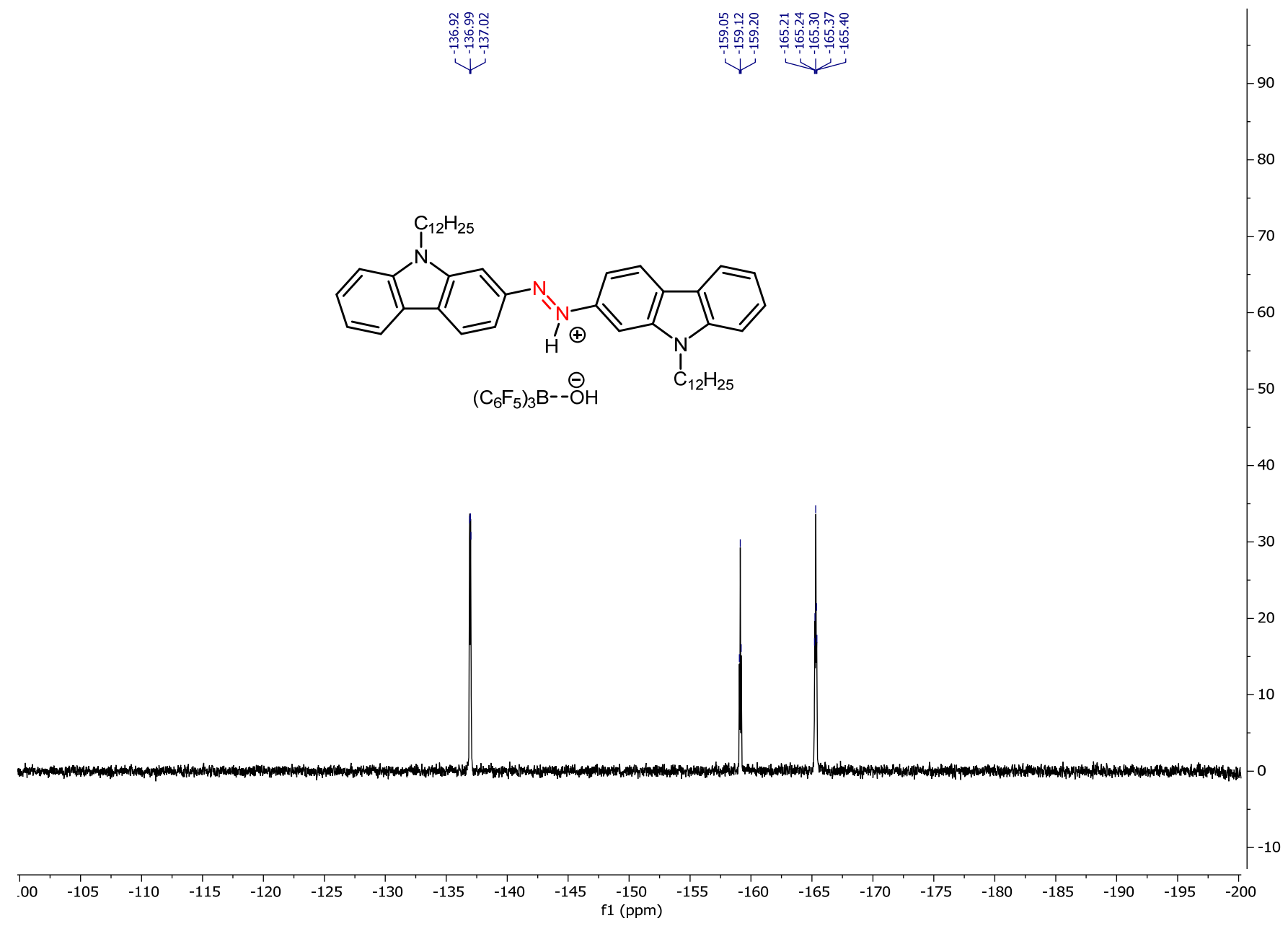

Figure S9. ${ }^{19} \mathrm{~F}$ NMR spectrum of $\mathbf{1 7 b}\left(282 \mathrm{MHz}, \mathrm{CDCl}_{3}\right)$. 


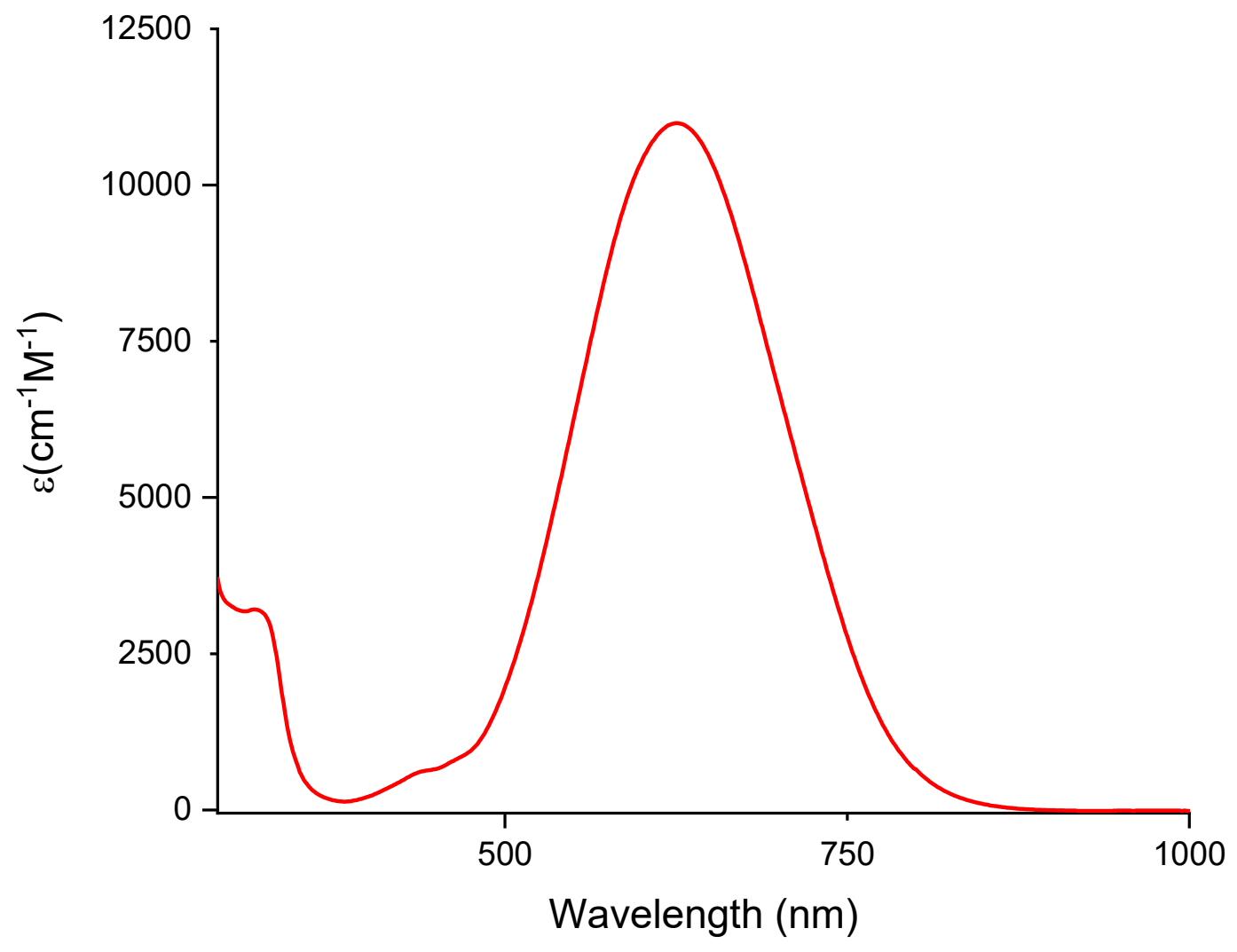

Figure S10. UV-Vis spectrum of $\mathbf{1 7 b}(0.143 \mathrm{mM})$ in $\mathrm{C}_{6} \mathrm{H}_{5} \mathrm{Cl}$. 


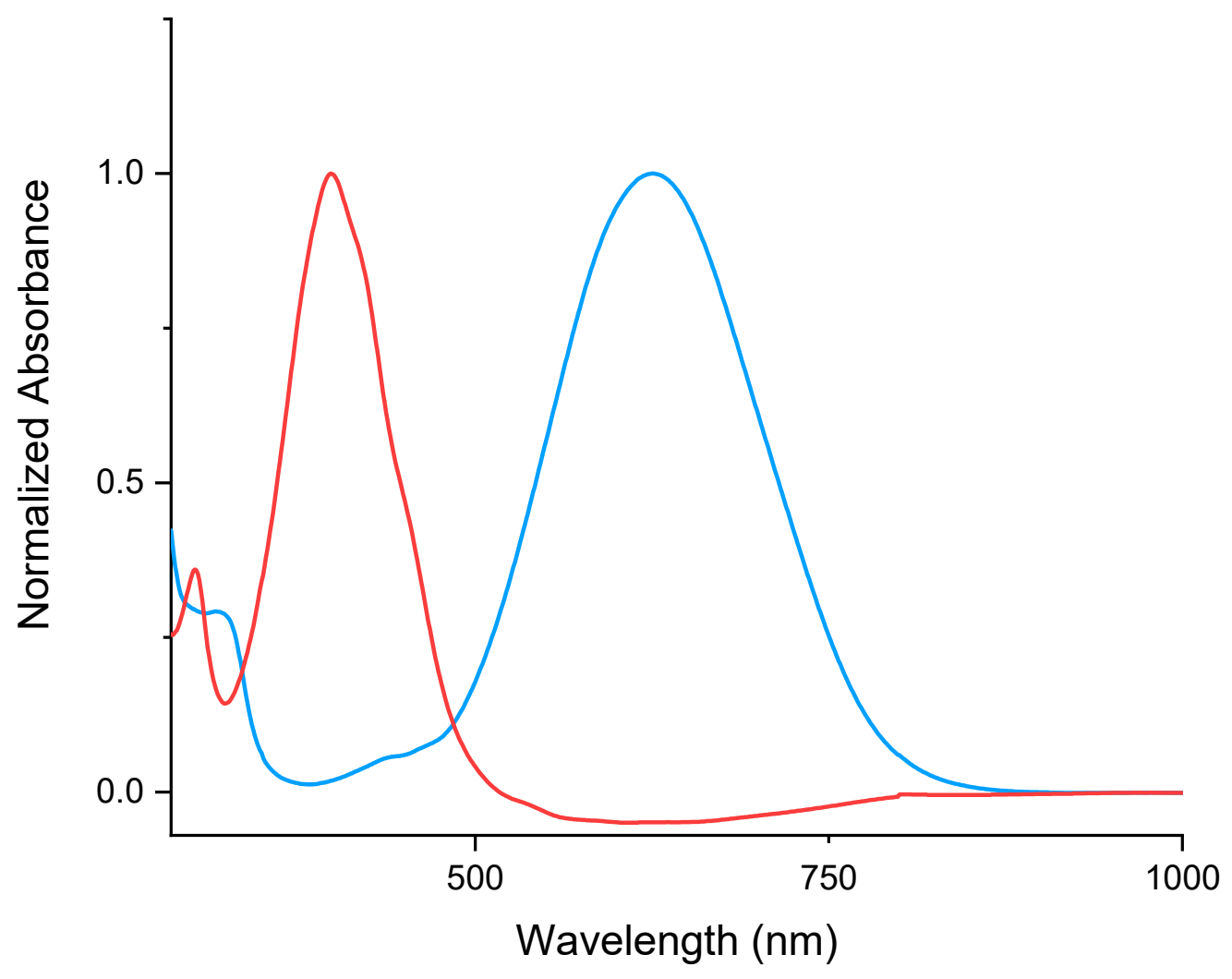

Figure S11. Normalized UV-Vis spectrum of $\mathbf{5 b}$ (red) and $\mathbf{1 7 b}$ (blue) in $\mathrm{C}_{6} \mathrm{H}_{5} \mathrm{Cl}$. 

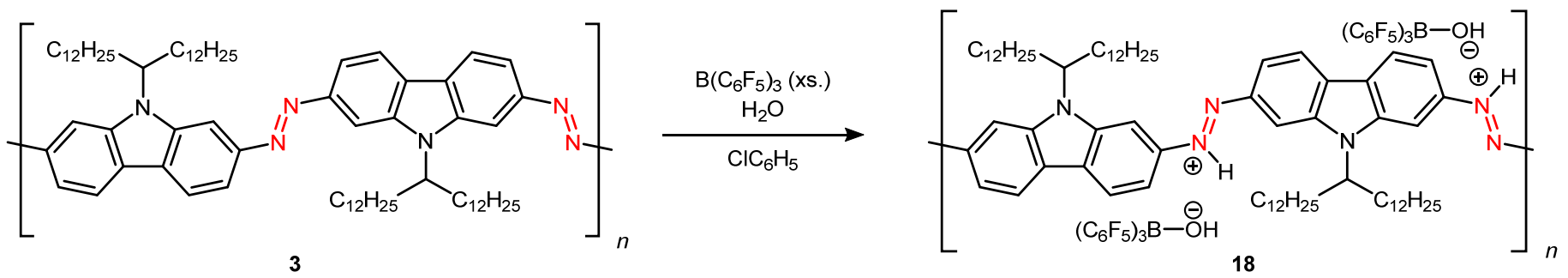

UV Vis titration of 3 using $\mathbf{B}\left(\mathbf{C}_{6} \mathbf{F}_{5}\right)_{3} \mathbf{( 1 8 )}$. A vial was charged with $\mathbf{3}(0.4 \mathrm{mg}, 0.73 \mu \mathrm{mol}, 1.0$ equiv) and $\mathrm{C}_{6} \mathrm{H}_{5} \mathrm{Cl}(20 \mathrm{~mL})$. An aliquot $(3 \mathrm{~mL})$ was taken, and $\mathrm{B}\left(\mathrm{C}_{6} \mathrm{~F}_{5}\right) 3(0.15 \mathrm{M}$ in $1.0 \mathrm{~mL})$ was titrated into the solution of 3.

${ }^{1} \mathrm{H}$ NMR of $18\left(400 \mathrm{MHz}, 100{ }^{\circ} \mathrm{C}\right.$, toluene- $\left.d_{8}\right) \delta 8.44(\mathrm{~s}, 2 \mathrm{H}), 8.08(\mathrm{q}, \mathrm{J}=8.9 \mathrm{~Hz}, 4 \mathrm{H}), 6.03$ (ddt, J = 14.2, 7.0, 3.6 Hz, 2H), $4.68(\mathrm{~s}, 1 \mathrm{H}), 2.41(\mathrm{~s}, 2 \mathrm{H}), 1.92(\mathrm{~s}, 2 \mathrm{H}), 1.20(\mathrm{~d}, \mathrm{~J}=23.2 \mathrm{~Hz}, 40 \mathrm{H}), 0.91-0.82(\mathrm{~m}, 6 \mathrm{H})$.

UV-Vis-NIR of $18\left(\mathrm{C}_{6} \mathrm{H}_{5} \mathrm{Cl}, 0.0326 \mathrm{mM}\right): \lambda_{\max }, \mathrm{nm}\left(\varepsilon, \mathrm{M}^{-1} \mathrm{~cm}^{-1}\right): 411(4,400), 748(\mathrm{sh}), 821(18,000)$

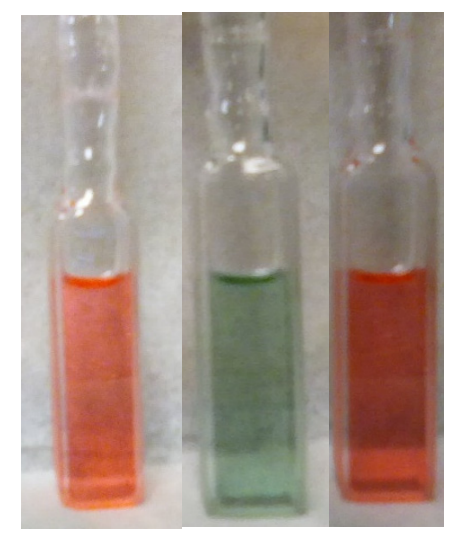

Figure S12. Pictures of (from left to right) 3, 18 (after $0.151 \mathrm{M}$ addition of $\left.\mathrm{B}\left(\mathrm{C}_{6} \mathrm{~F}_{5}\right)_{3}\right)$ and $\mathbf{1 8}$ after 1 drop pyridine in $\mathrm{C}_{6} \mathrm{H}_{5} \mathrm{Cl}$. 


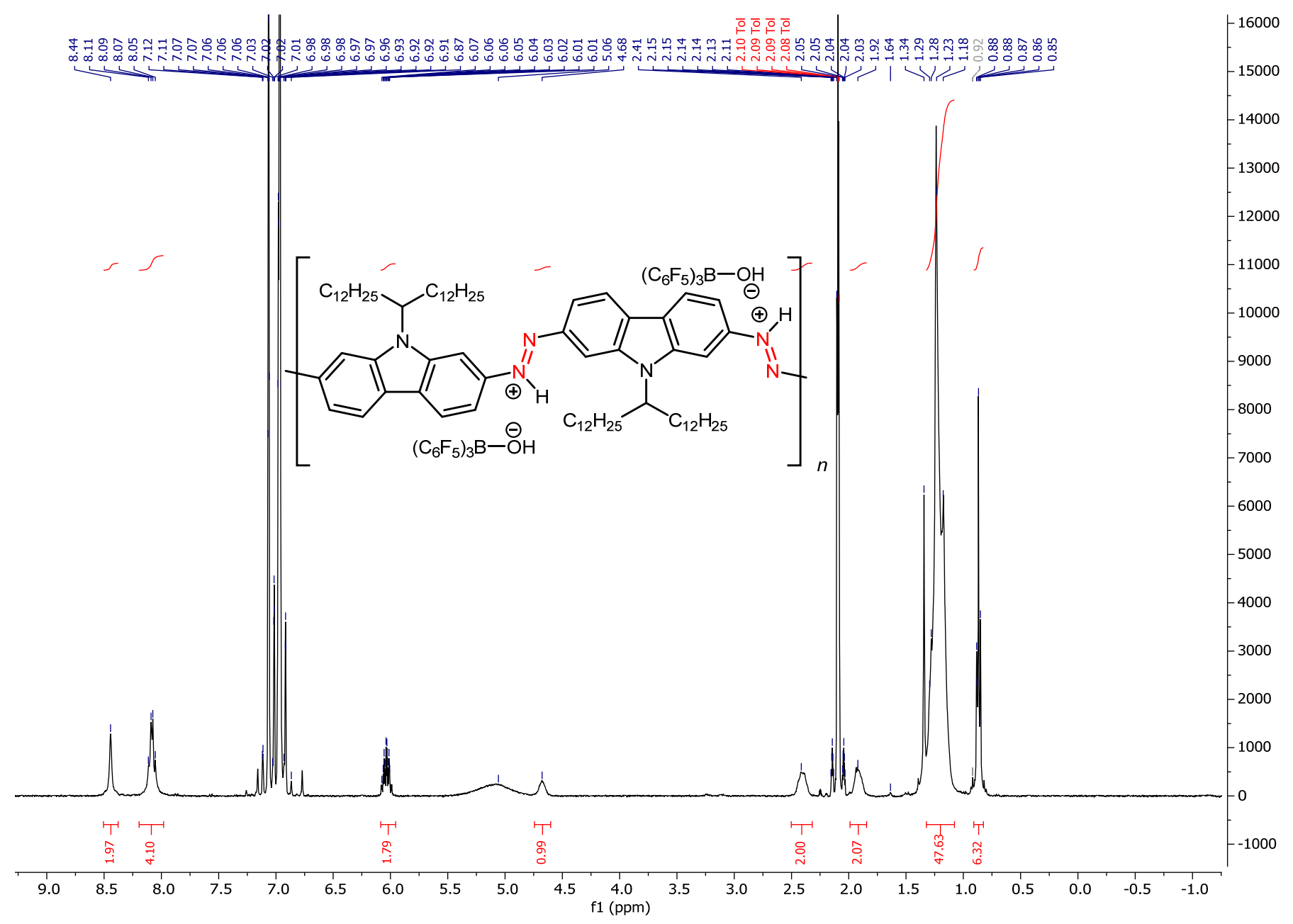

Figure S13. ${ }^{1} \mathrm{H}$ NMR spectrum of $18\left(400 \mathrm{MHz}, 100{ }^{\circ} \mathrm{C}\right.$, toluene- $\left.d_{8}\right)$. 


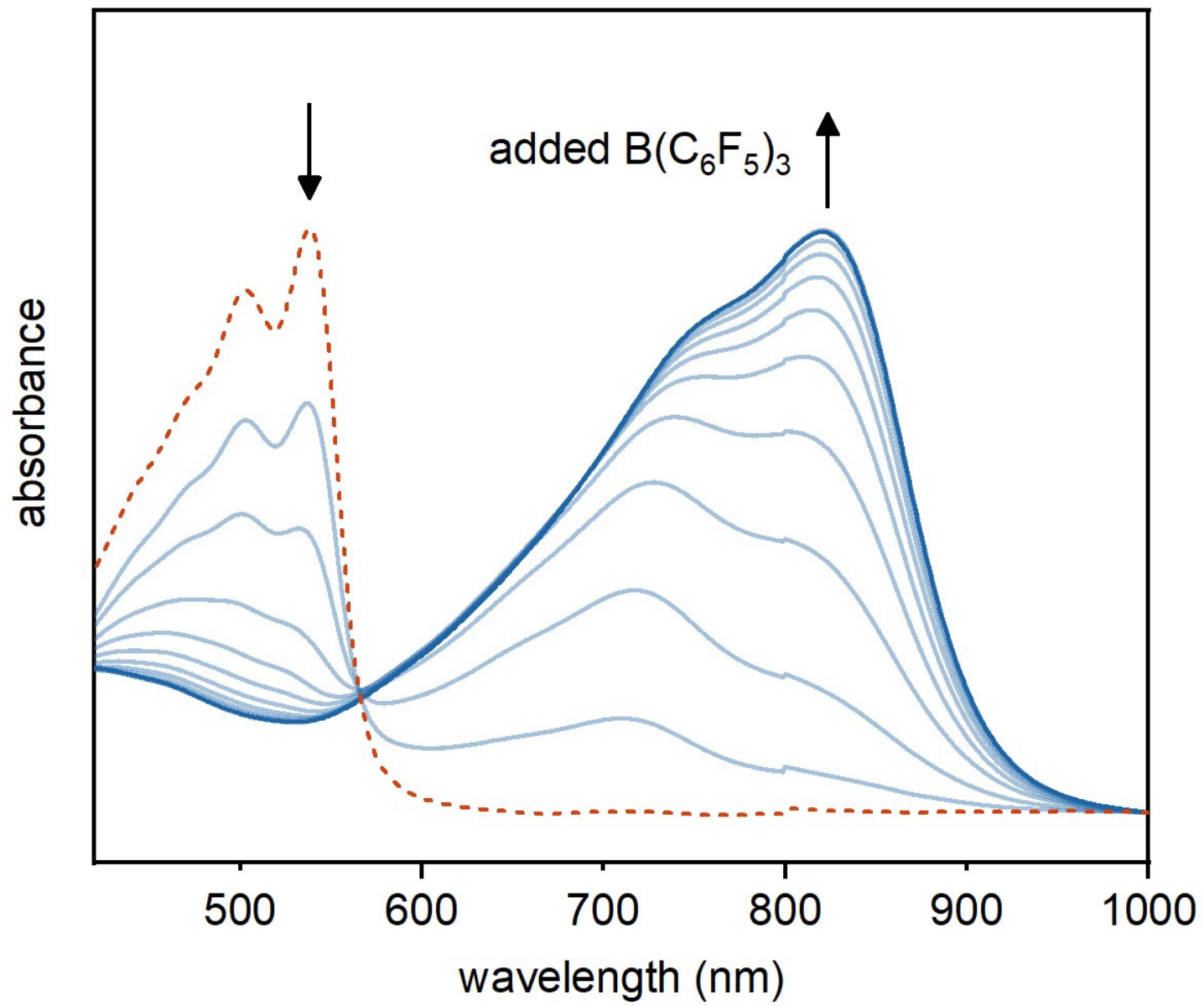

Figure S14. UV-Vis titration of $\mathrm{B}\left(\mathrm{C}_{6} \mathrm{~F}_{5}\right)_{3}(0.15 \mathrm{M})$ into $3(0.0326 \mathrm{mM})$ in $\mathrm{C}_{6} \mathrm{H}_{5} \mathrm{Cl}$. 


\section{Cyclic Voltammetry}

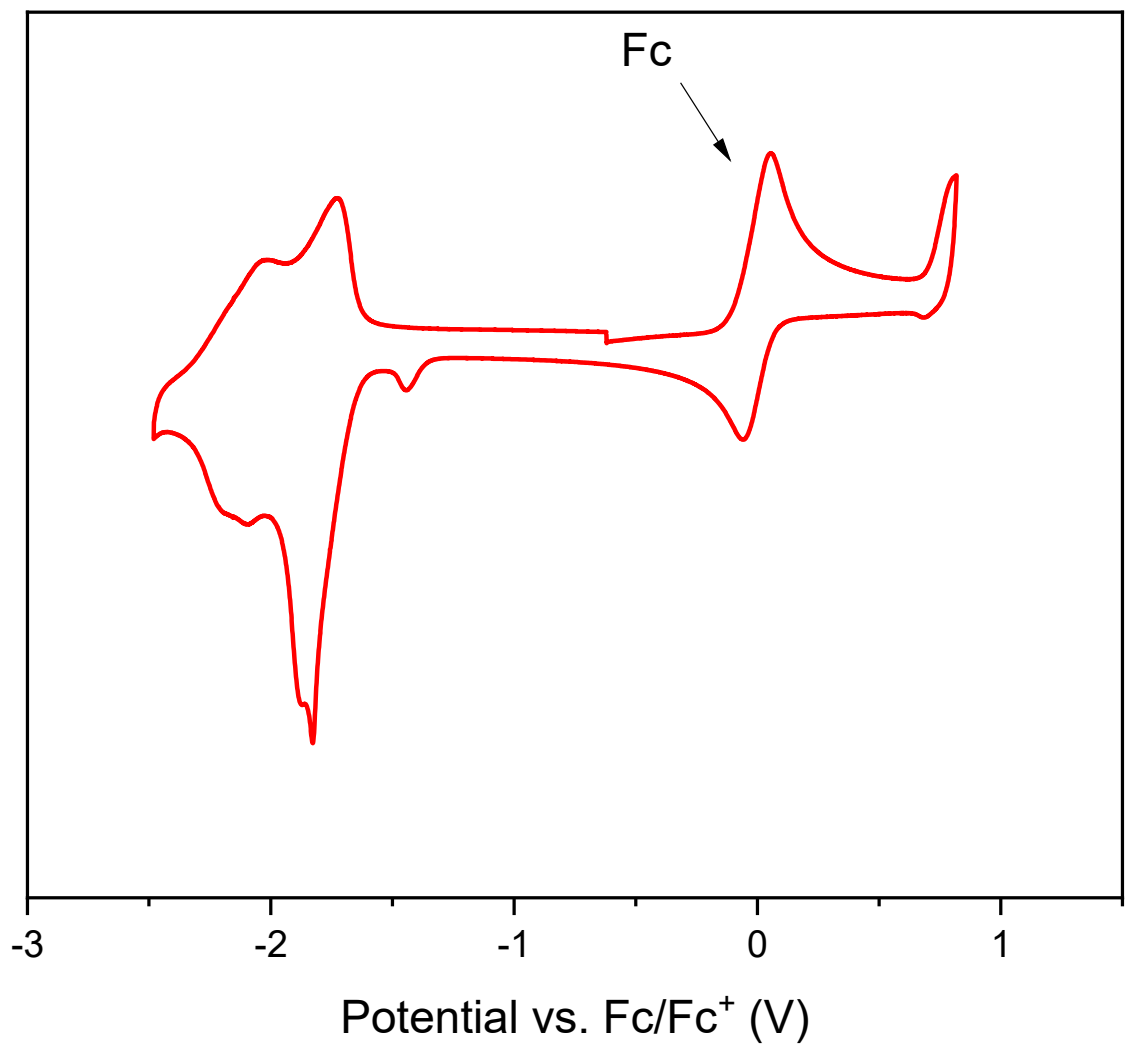

Figure S15. Cyclic voltammogram of $\mathbf{3}$. The voltammogram was taken by heating a solution of $\mathbf{3}$ and dropping it onto a glassy carbon working electrode. Supporting electrolyte: $0.3 \mathrm{M} \mathrm{TBAPF}_{6}$ in THF; 100 $\mathrm{mV} / \mathrm{s}$ scan rate. Scans were started at the open-circuit potential and scanned in the anodic direction. 


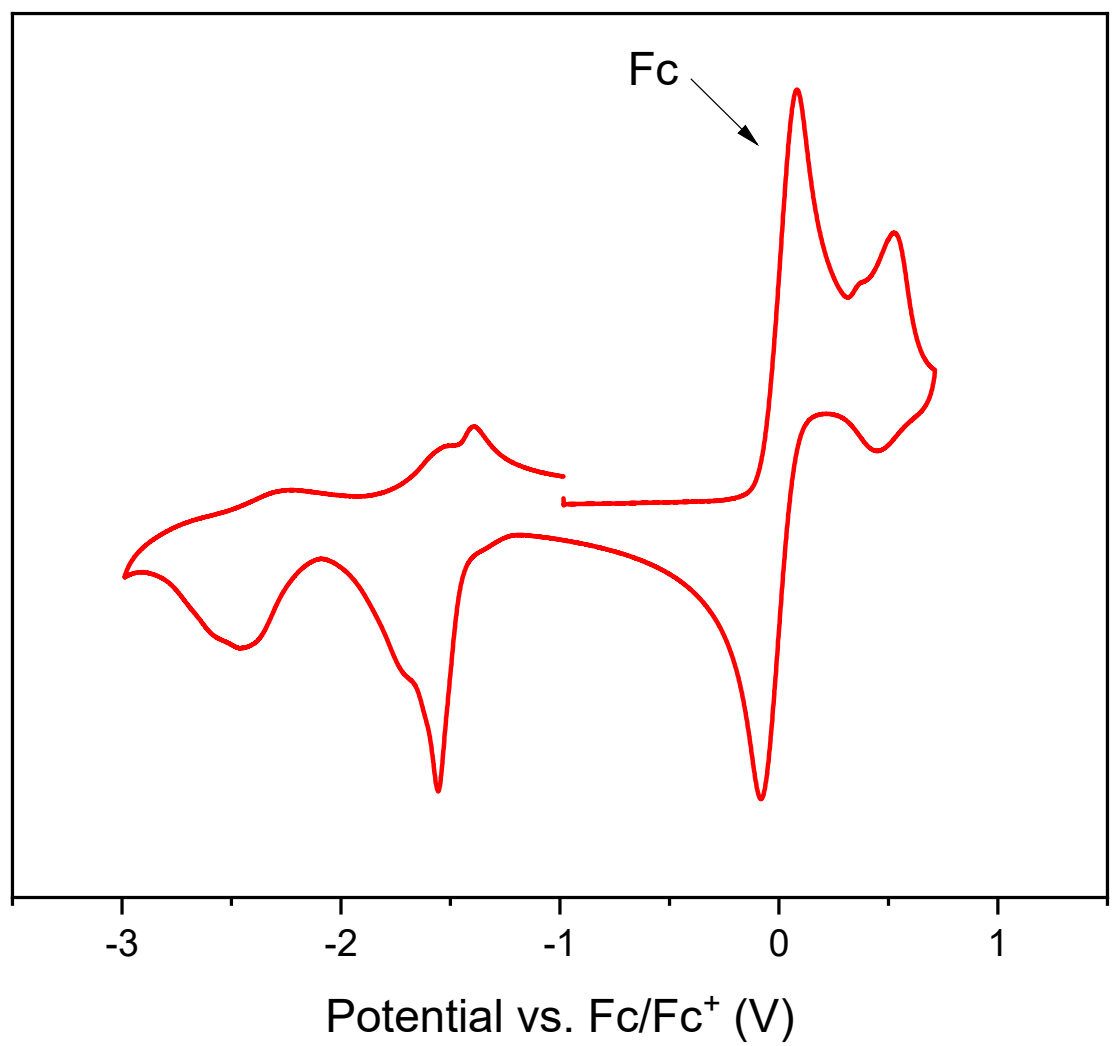

Figure S16. Cyclic voltammogram of 7. The voltammogram was taken by heating a solution of $\mathbf{7}$ and dropping it onto a glassy carbon working electrode. Supporting electrolyte: $0.3 \mathrm{M} \mathrm{TBAPF}_{6}$ in THF; 100 $\mathrm{mV} / \mathrm{s}$ scan rate. Scans were started at the open-circuit potential and scanned in the anodic direction. 


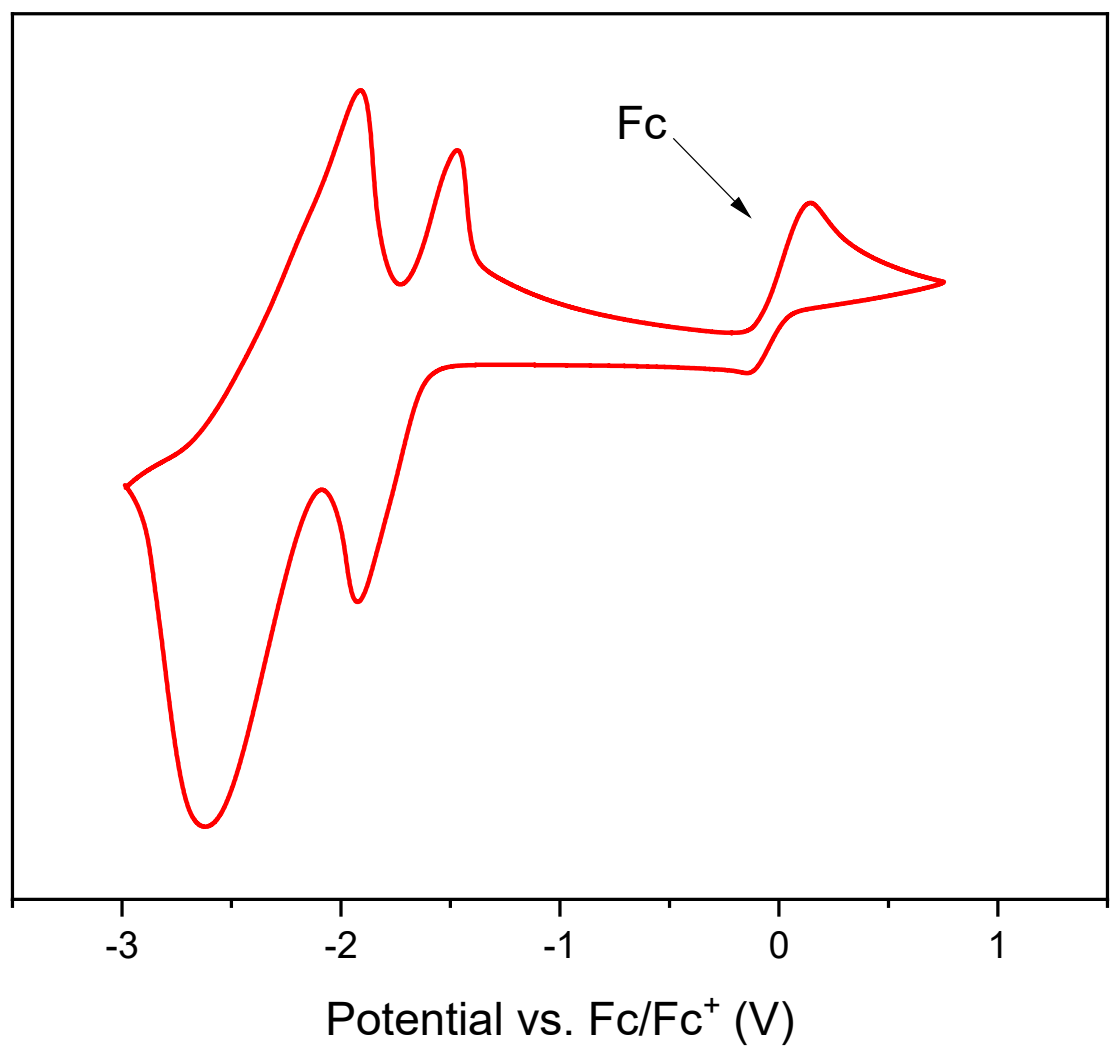

Figure S17. Cyclic voltammogram of $\mathbf{8}$. The voltammogram was taken by heating a solution of $\mathbf{8}$ and dropping it onto a glassy carbon working electrode. Supporting electrolyte: $0.3 \mathrm{M} \mathrm{TBAPF}_{6}$ in THF; 100 $\mathrm{mV} / \mathrm{s}$ scan rate. Scans were started at the open-circuit potential and scanned in the anodic direction. 


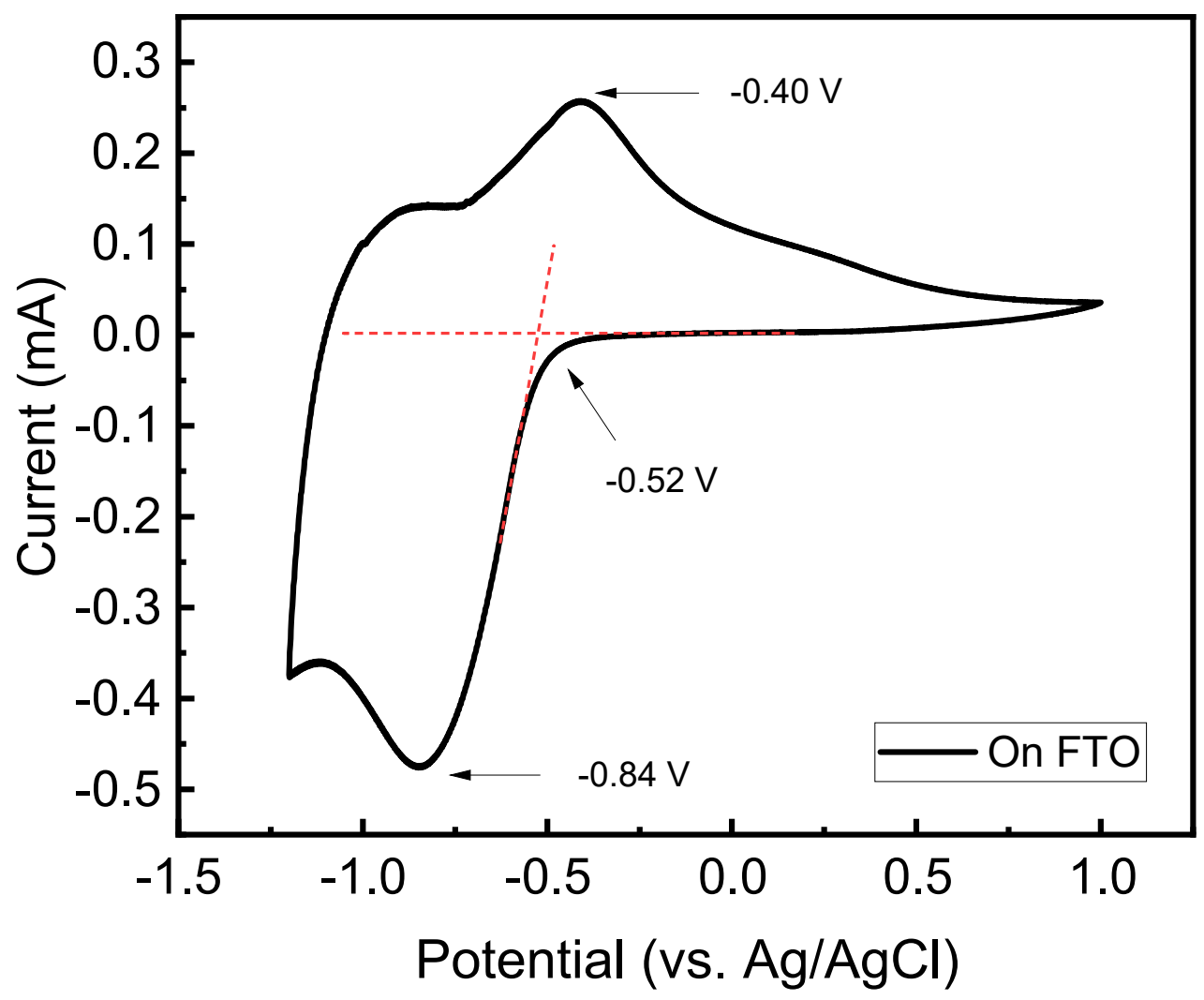

Figure S18. Cyclic voltammogram for a thin film of $\mathbf{1 0}$ spin coated on FTO/glass substrate.

Voltammograms taken in a $0.2 \mathrm{M} \mathrm{TBAPF}_{6}$ in propylene carbonate (PC) with a $50 \mathrm{mV} / \mathrm{s}$ scan rate. The $\mathrm{E}_{1 / 2}$ value for $\mathbf{1 0}$ is $-0.62 \mathrm{~V}$ vs. $\mathrm{Ag} / \mathrm{AgCl}$. 

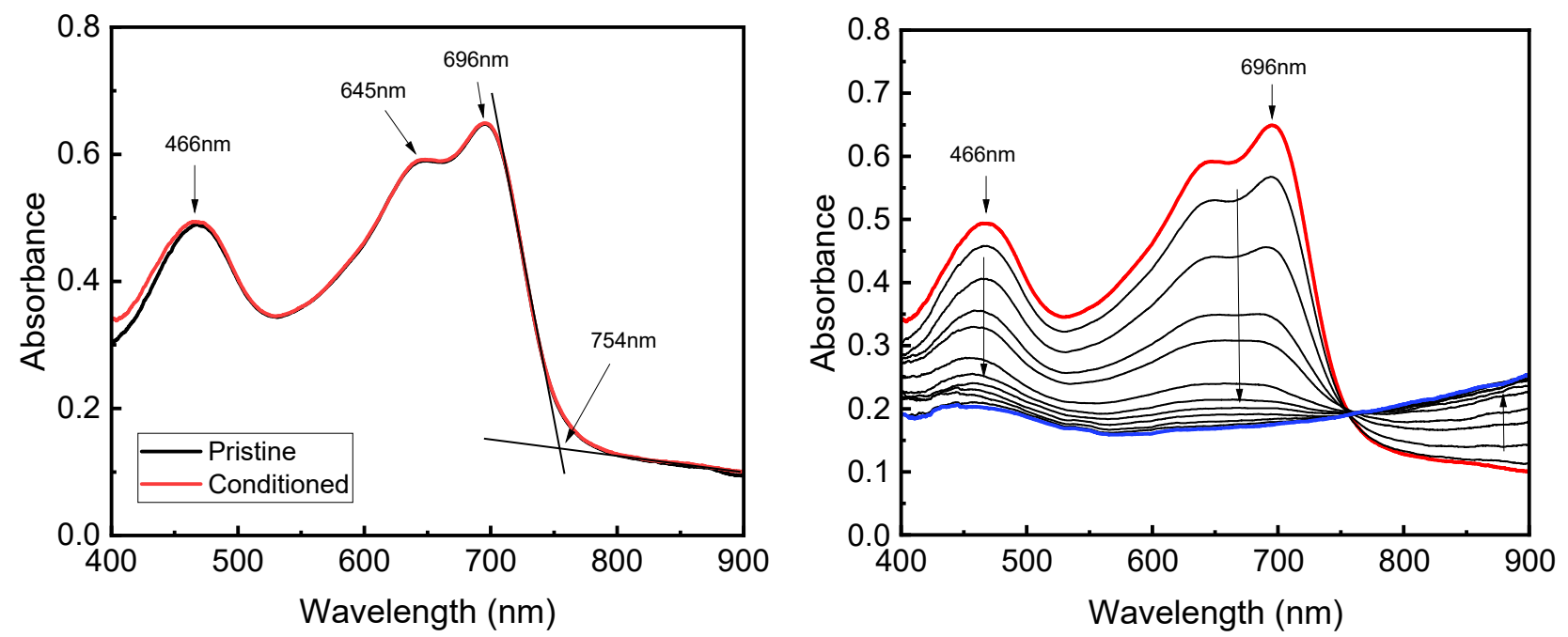

Figure S19. Spectroelectrochemistry for a thin film of 10 spin coated onto FTO/glass substrate in $0.2 \mathrm{M}$ $\mathrm{TBAPF}_{6} /$ Propylene carbonate. UV-Vis Spectra of prisitine film and electrochemical conditioned film (left). Spectroelectrochemistry of $\mathbf{1 0}$ (red) subjected to $20 \mathrm{mV}$ potential increments from $-0.4 \mathrm{~V}$ to -0.7 $\mathrm{V}$ vs Ag/AgCl (right). The polymer film bleaches at $-0.7 \mathrm{~V}$ (blue). 


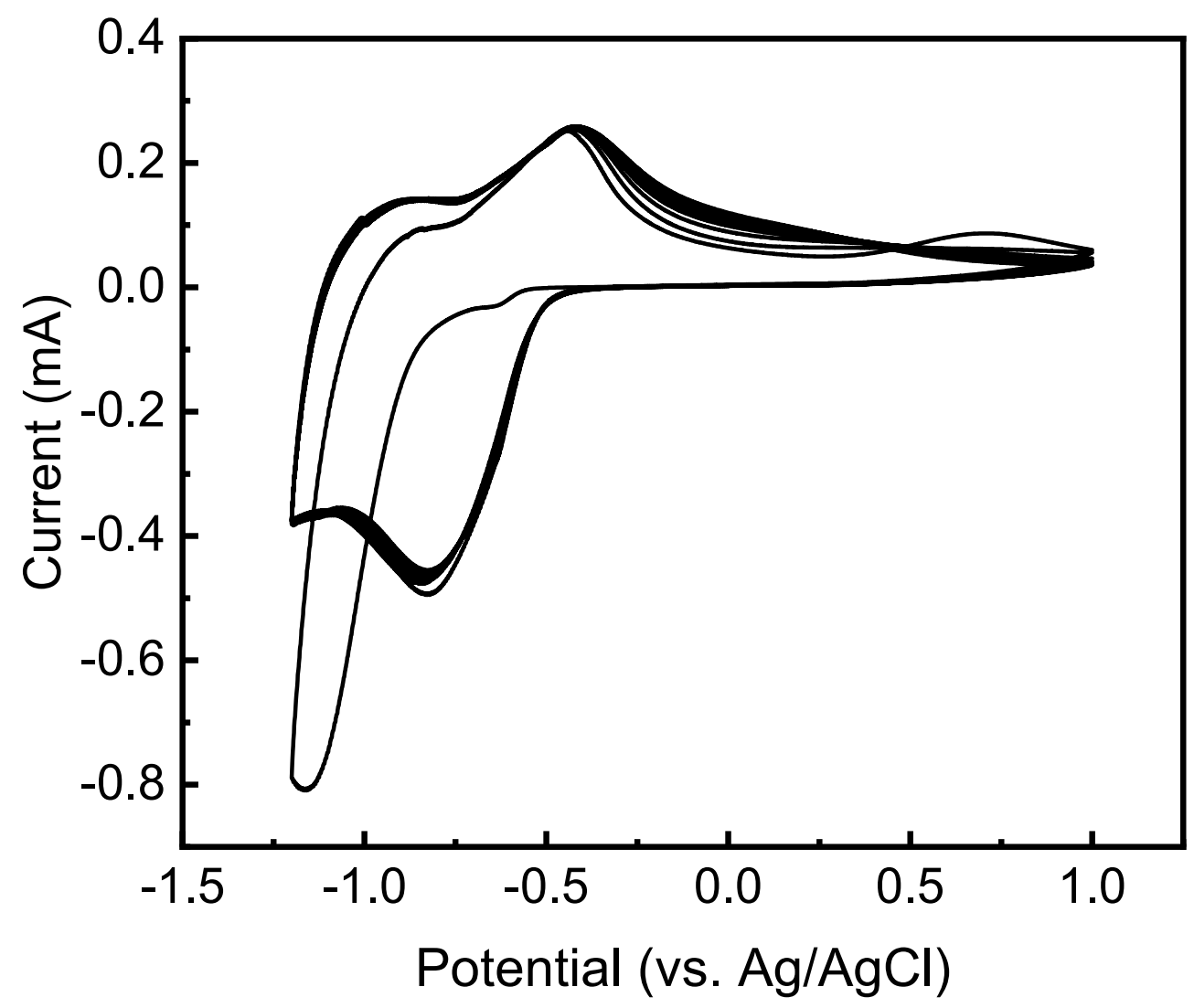

Figure S20. Cyclic voltammogram of $\mathbf{1 0}$ deposited on Fluorine-doped tin oxide (FTO) substrate after 10 continuous cycles under $\mathrm{N}_{2}$ atmosphere. Voltammograms taken in a $0.2 \mathrm{M} \mathrm{TBAPF}_{6}$ in propylene carbonate (PC) with a $50 \mathrm{mV} / \mathrm{s}$ scan rate. The $\mathrm{E}_{1 / 2}$ value for 10 is $-0.62 \mathrm{~V} \mathrm{vs.} \mathrm{Ag} / \mathrm{AgCl}$. 


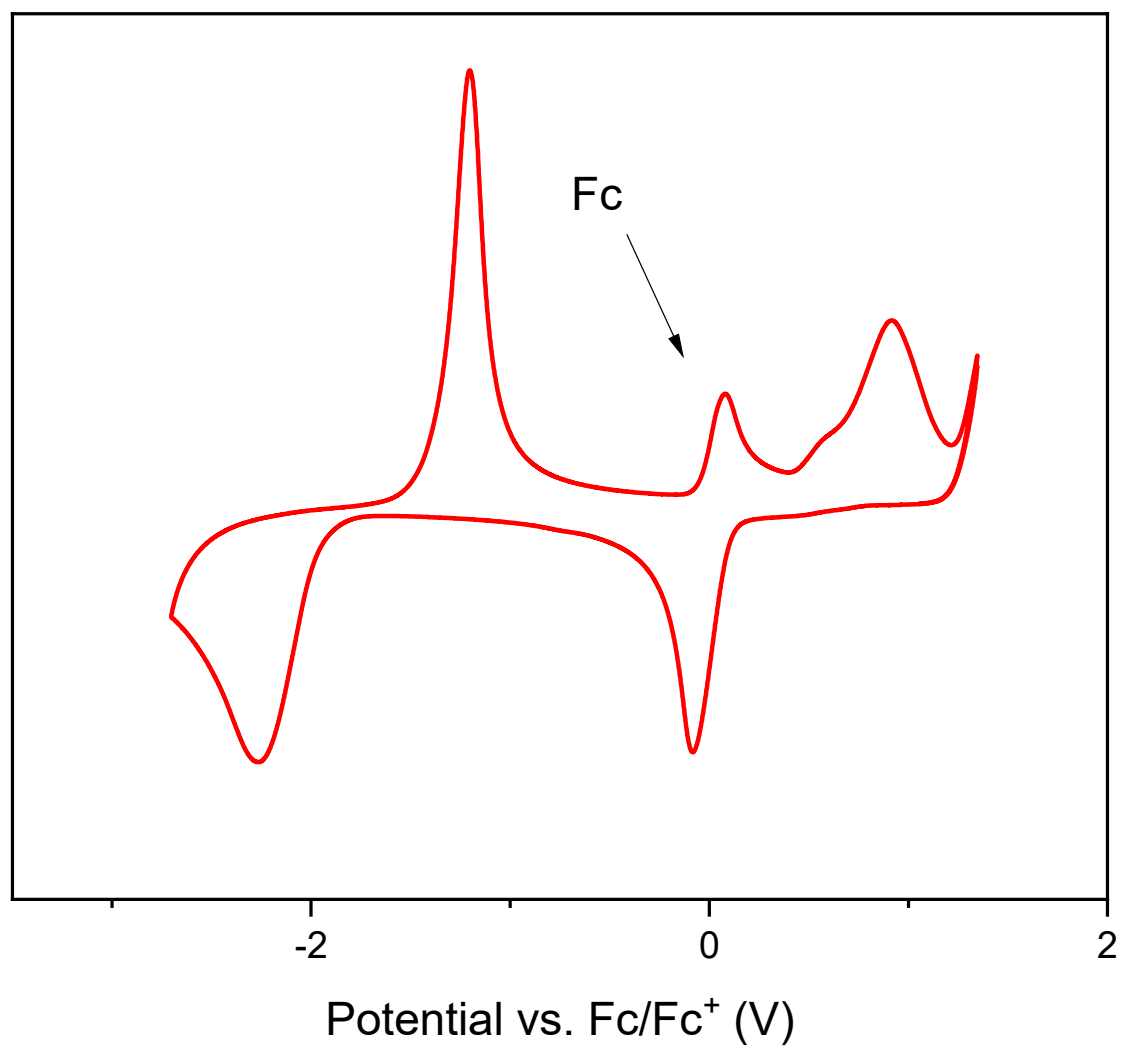

Figure S21. Cyclic voltammogram of 11. The voltammogram was taken by heating a solution of $\mathbf{1 1}$ and dropping it onto a glassy carbon working electrode. Supporting electrolyte: $0.3 \mathrm{M} \mathrm{TBAPF}_{6}$ in THF; $100 \mathrm{mV} / \mathrm{s}$ scan rate. Scans were started at the open-circuit potential and scanned in the anodic direction. 


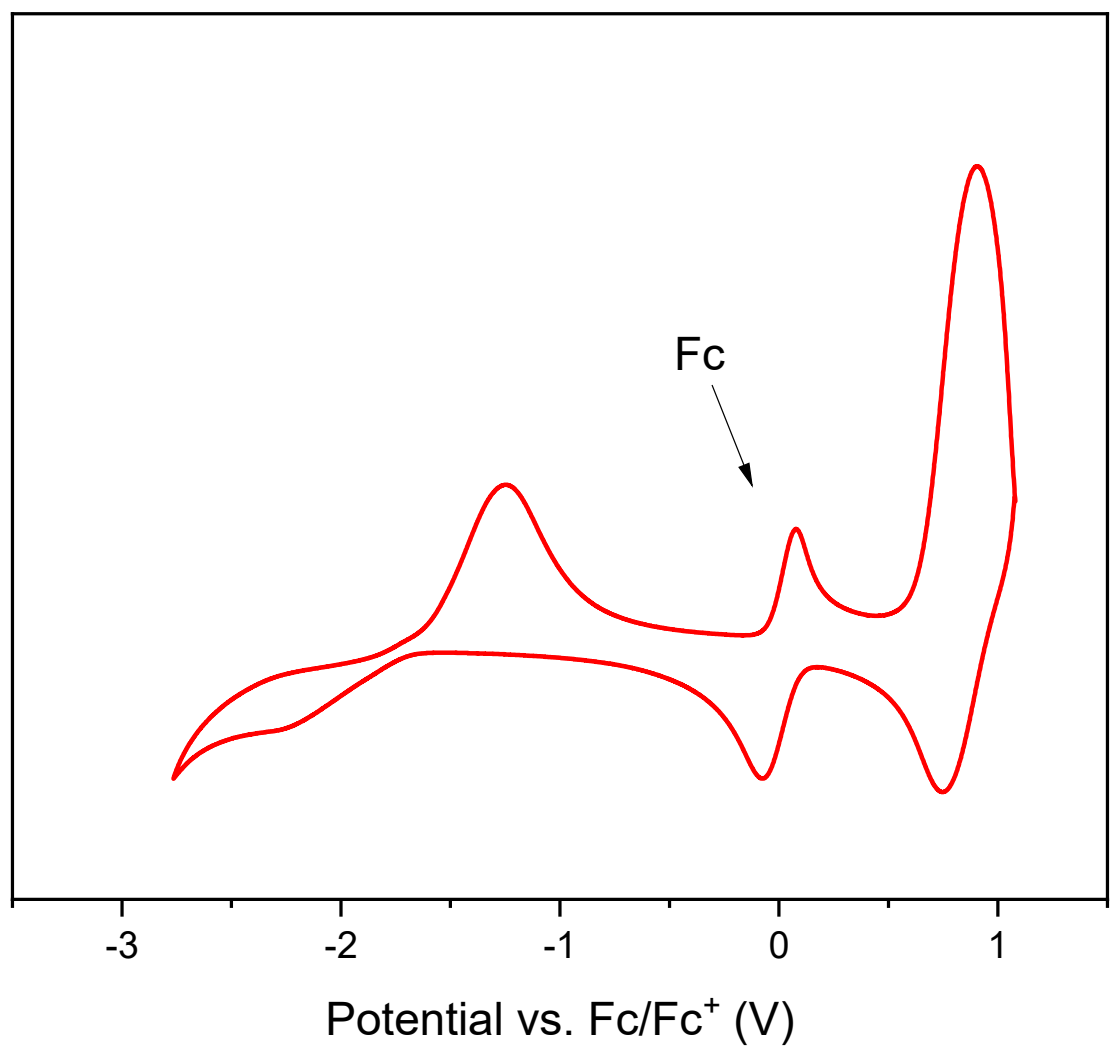

Figure S22. Cyclic voltammogram of 12. The voltammogram was taken by heating a solution of $\mathbf{1 2}$ and dropping it onto a glassy carbon working electrode. Supporting electrolyte: $0.3 \mathrm{M} \mathrm{TBAPF}_{6}$ in THF; $100 \mathrm{mV} / \mathrm{s}$ scan rate. Scans were started at the open-circuit potential and scanned in the anodic direction. 


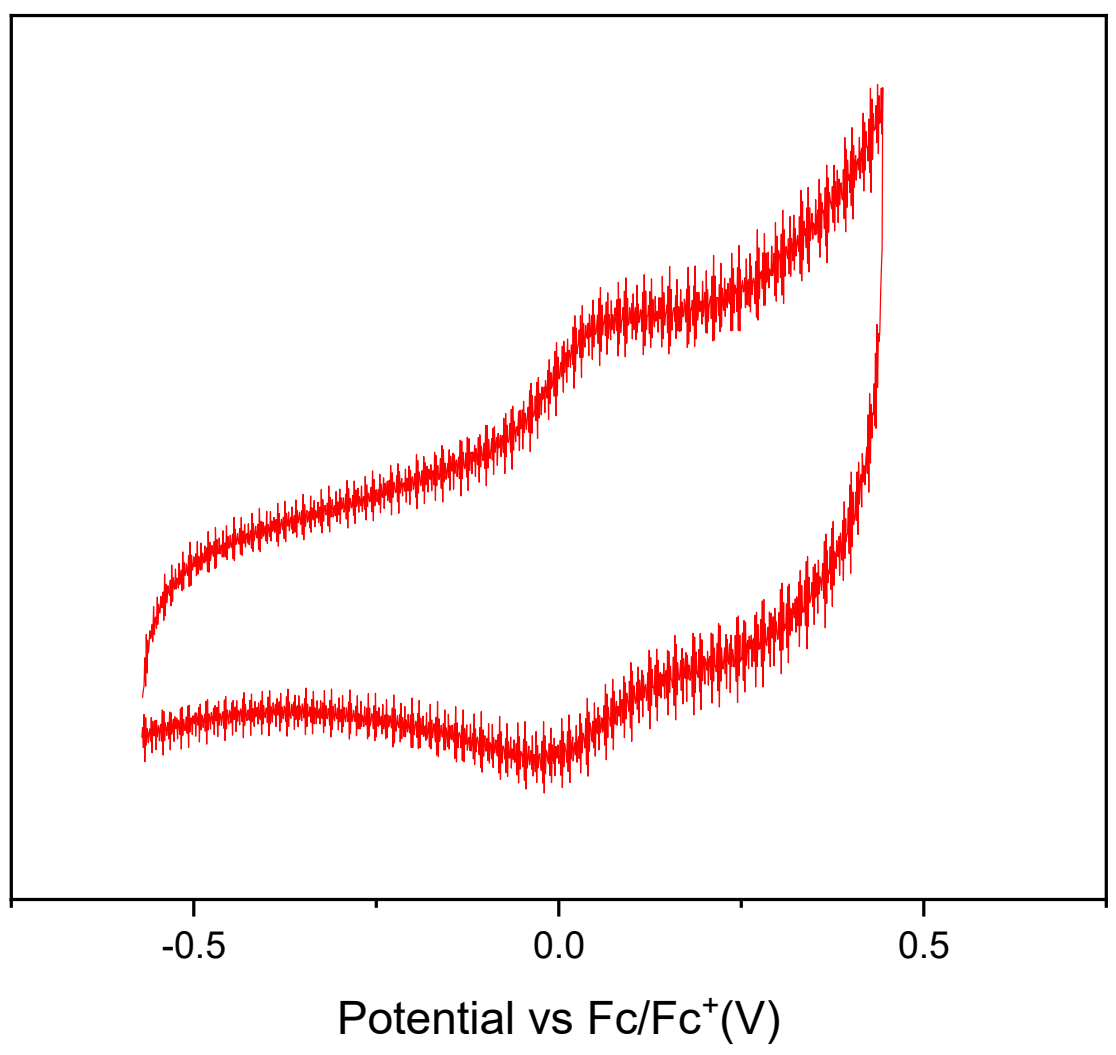

Figure S23. Cyclic voltammogram isolated in the ferrocene couple for 15. The voltammogram was taken by heating a solution of $\mathbf{1 5}$ and dropping it onto a glassy carbon working electrode. Supporting electrolyte: $0.3 \mathrm{M} \mathrm{TBAPF}_{6}$ in THF; $100 \mathrm{mV} / \mathrm{s}$ scan rate. Scans were started at the open-circuit potential and scanned in the cathodic direction. The $\mathrm{E}_{1 / 2}$ value for $\mathbf{1 5}$ is $0.02 \mathrm{~V}$ vs. $\mathrm{Fc} / \mathrm{Fc}^{+}$. 


\section{Photoswitching Experiments}

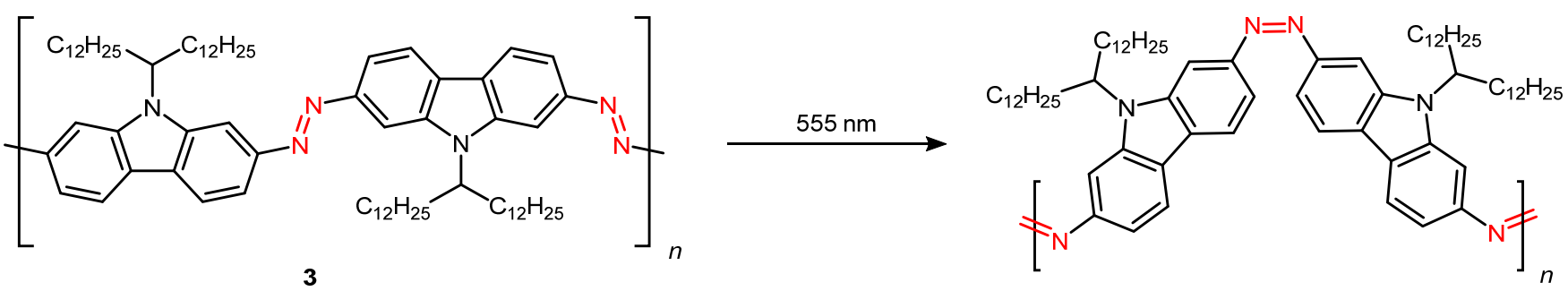

PolyAzoCarbazole (3). A vial was charged with $3(0.4 \mathrm{mg})$ and $\mathrm{C}_{6} \mathrm{H}_{5} \mathrm{Cl}(20 \mathrm{~mL})$. An aliquot (3 mL) was added to a cuvette, irradiated for $30 \mathrm{~s}$ at $555 \mathrm{~nm}$, and quickly transferred to a UV-Vis instrument to obtain measurements.

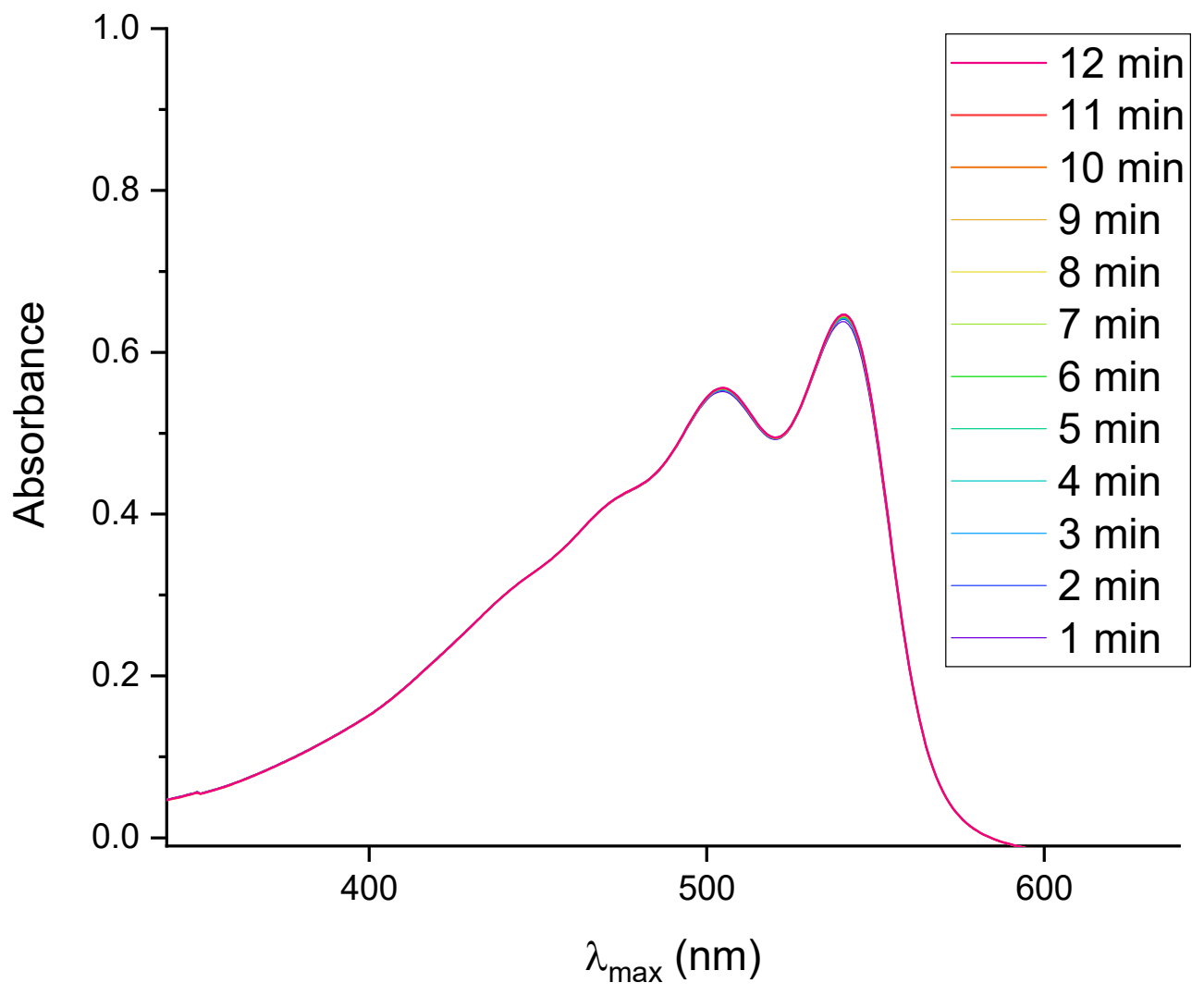

Figure S24. UV-Vis spectrum of photoisomerization of $3(0.0326 \mathrm{mM})$ in $\mathrm{C}_{6} \mathrm{H}_{5} \mathrm{Cl}$. 


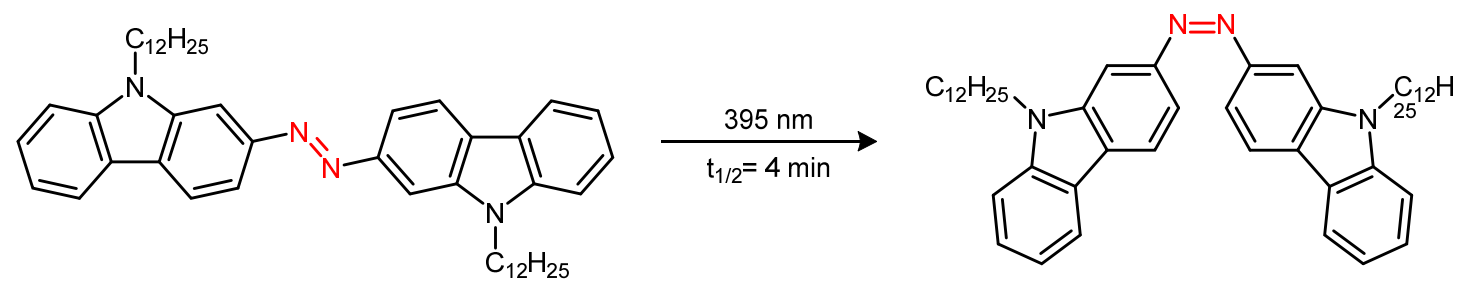

5b

Azocarbazole (5b). A vial was charged with $\mathbf{5 b}(0.4 \mathrm{mg})$ and $\mathrm{CH}_{2} \mathrm{Cl}_{2}(20 \mathrm{~mL})$. An aliquot ( $\left.3 \mathrm{~mL}\right)$ was added to a cuvette, irradiated for $30 \mathrm{~s}$ at $395 \mathrm{~nm}$, and quickly transferred to a UV-Vis instrument to obtain measurements.

UV-Vis-NIR ( $\left.\mathrm{CH}_{2} \mathrm{Cl}_{2}, 0.028 \mathrm{mM}\right): \lambda_{\max }, \mathrm{nm}\left(\varepsilon, \mathrm{M}^{-1} \mathrm{~cm}^{-1}\right): 264(62,000), 303(25,000), 393(18,000)$.

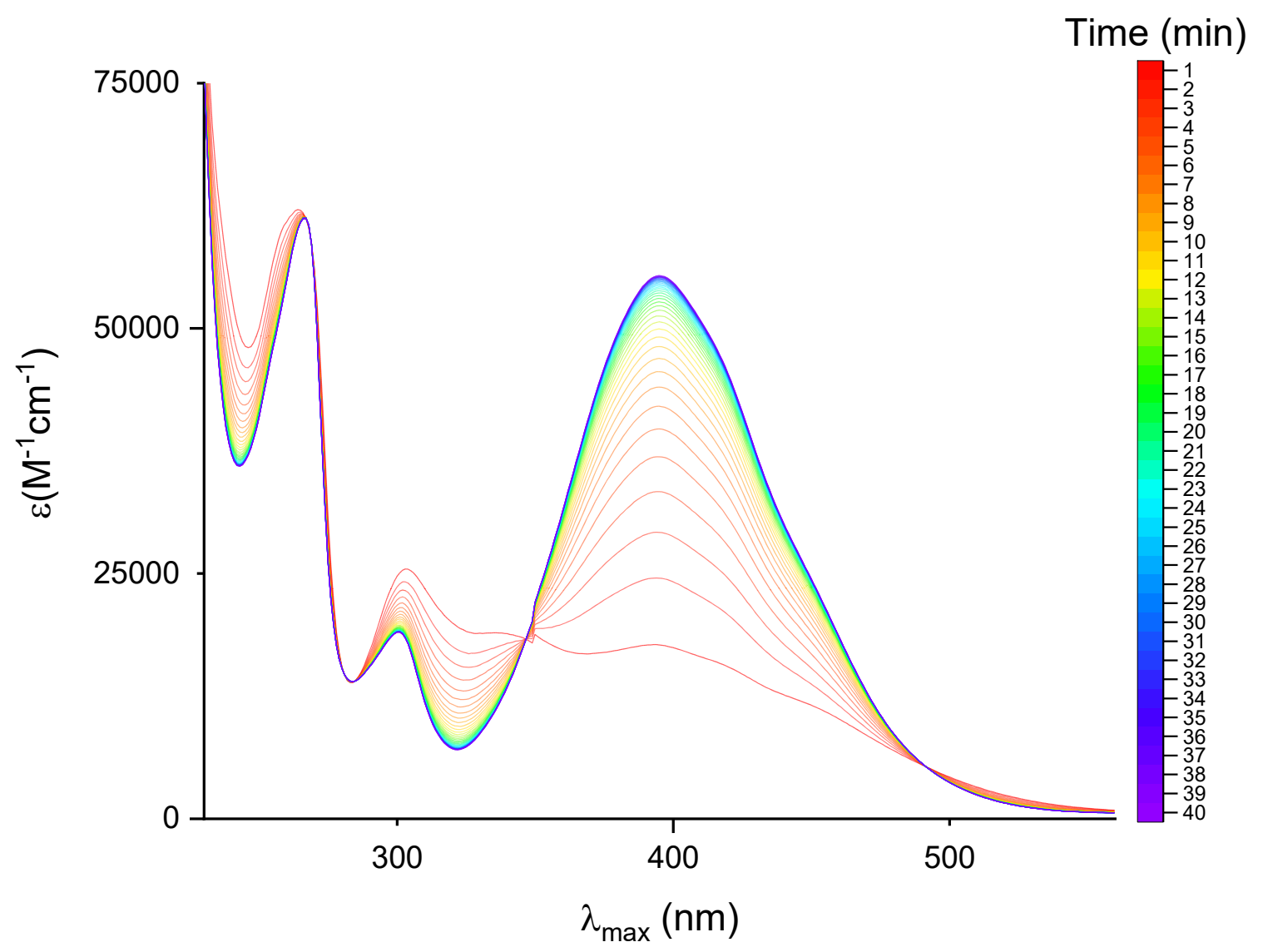

Figure S25. UV-Vis spectrum of photoisomerization of $\mathbf{5 b}(0.028 \mathrm{mM})$ in $\mathrm{CH}_{2} \mathrm{Cl}_{2}$. 


\section{Thermogravimetric Analysis}

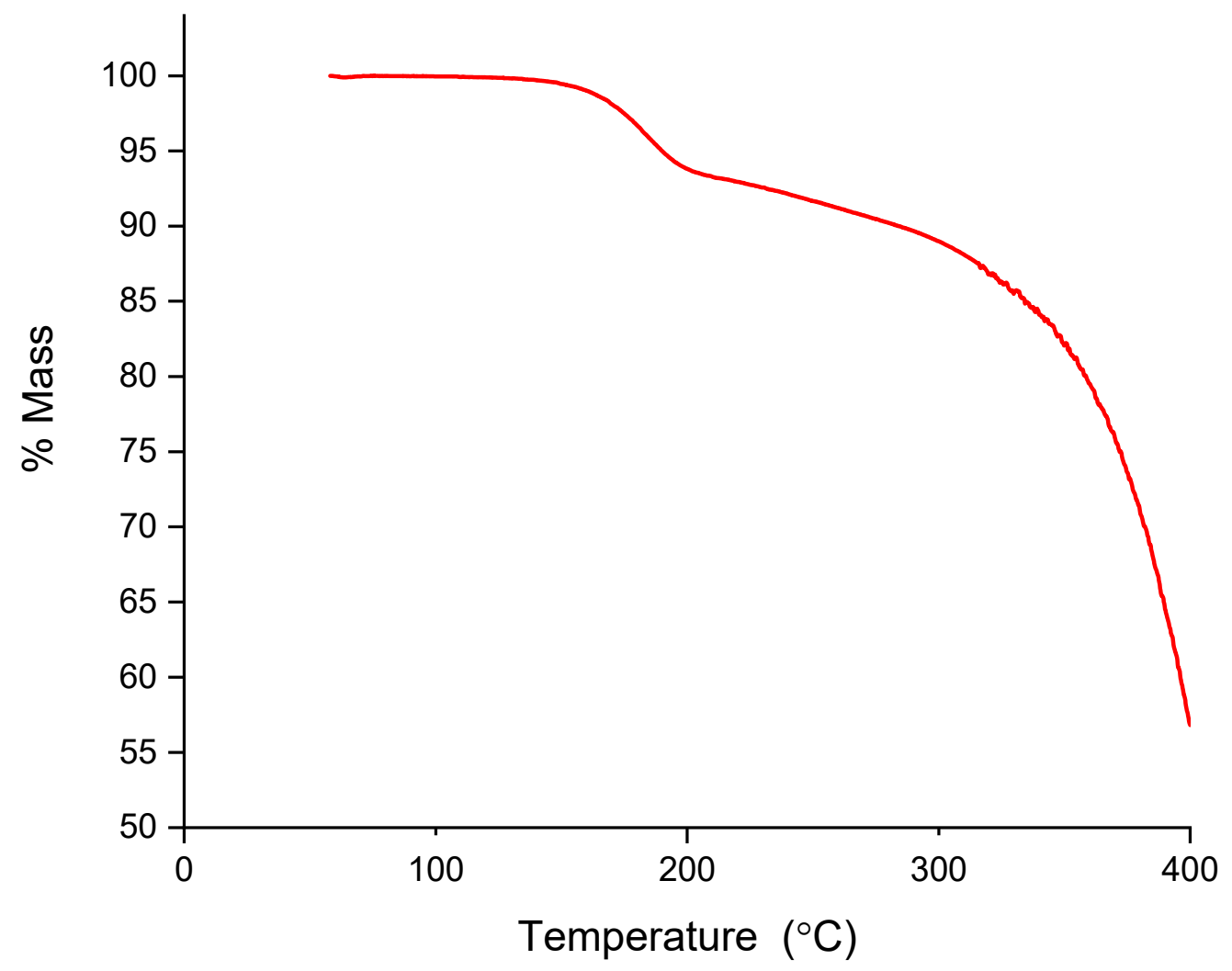

Figure S26. Thermogravimetric analysis of $\mathbf{S 1 0}$ at $20^{\circ} \mathrm{C}$ per minute. 


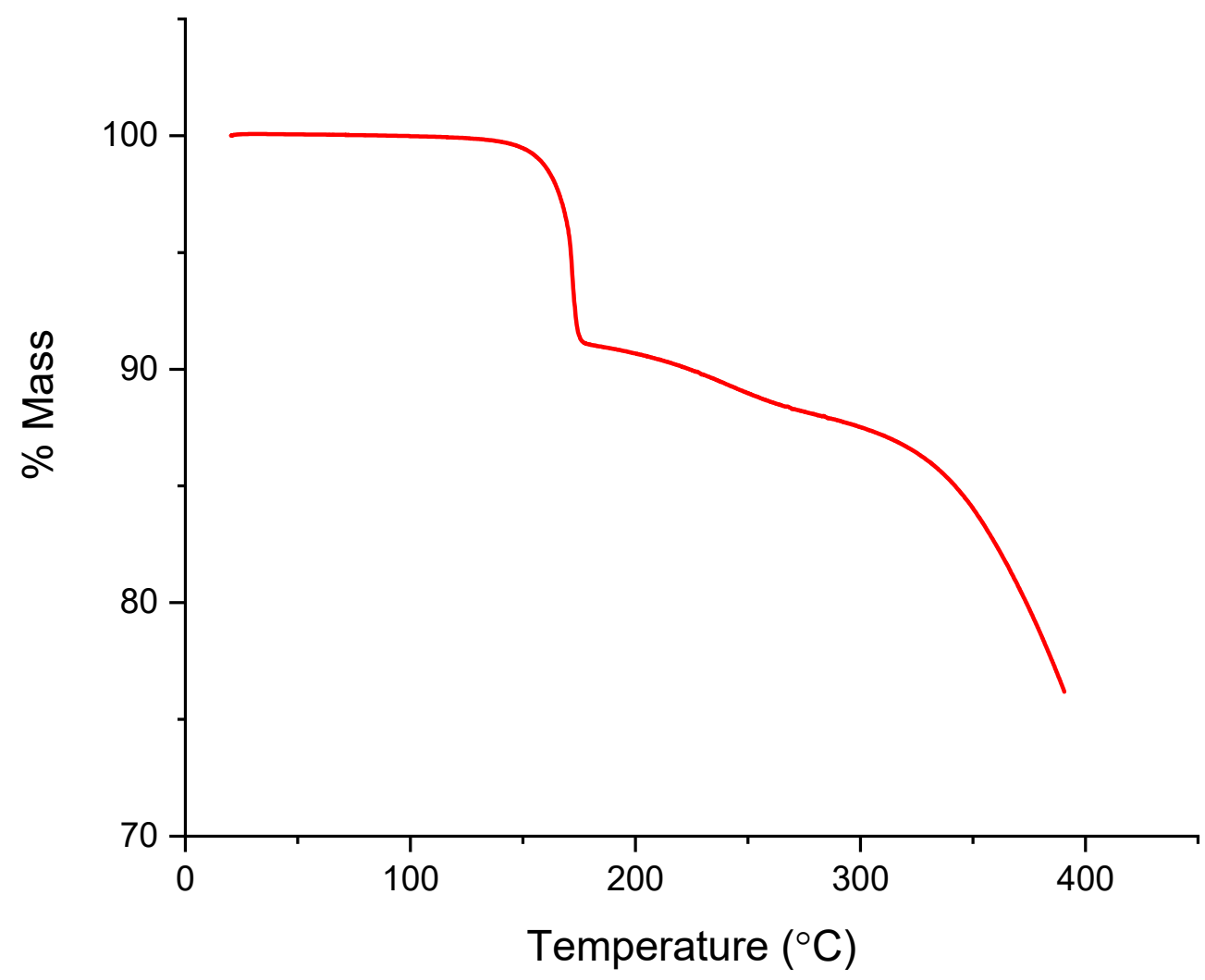

Figure S27. Thermogravimetric analysis of 2 at $20^{\circ} \mathrm{C}$ per minute. 


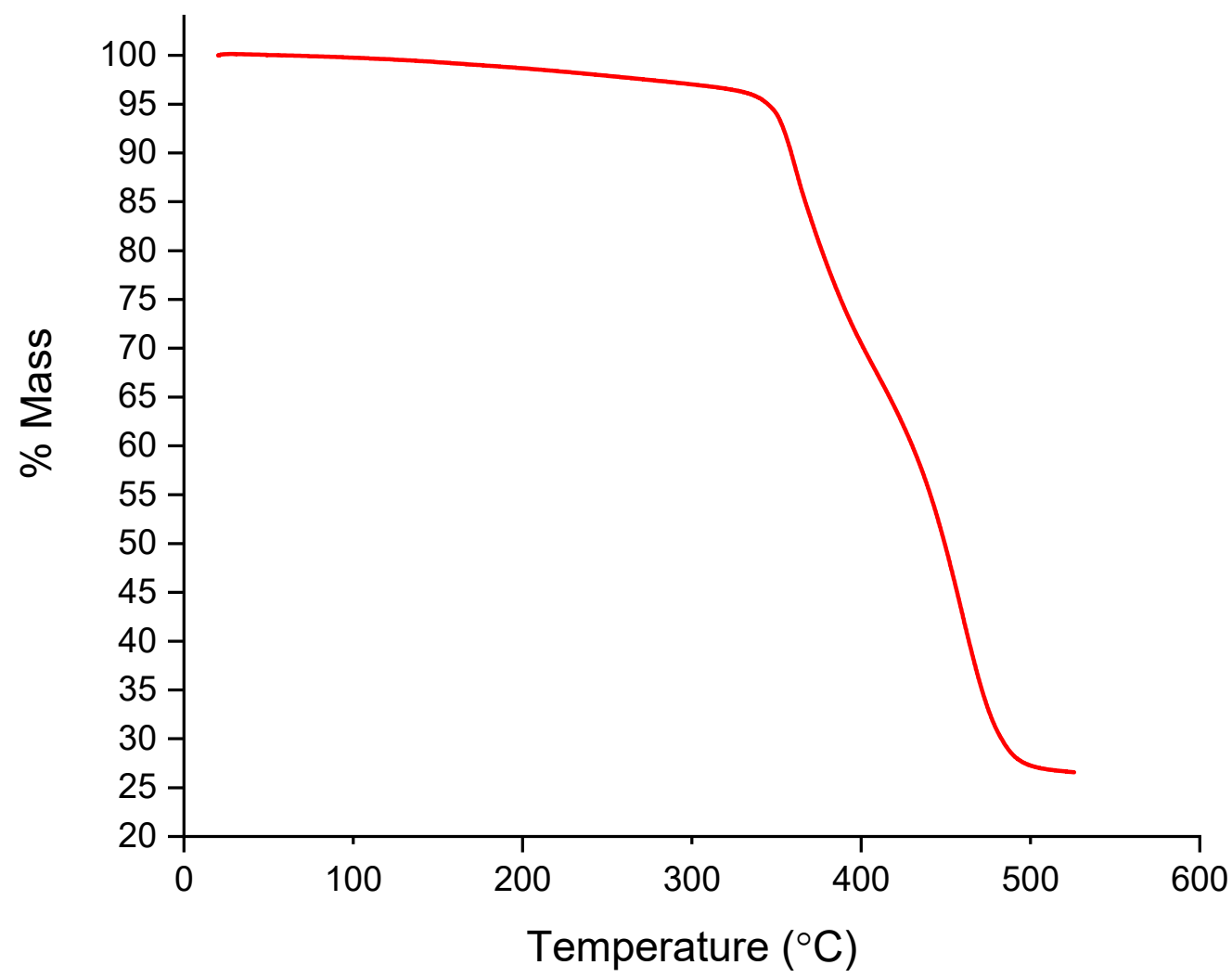

Figure S28. Thermogravimetric analysis of 3 at $20^{\circ} \mathrm{C}$ per minute. 


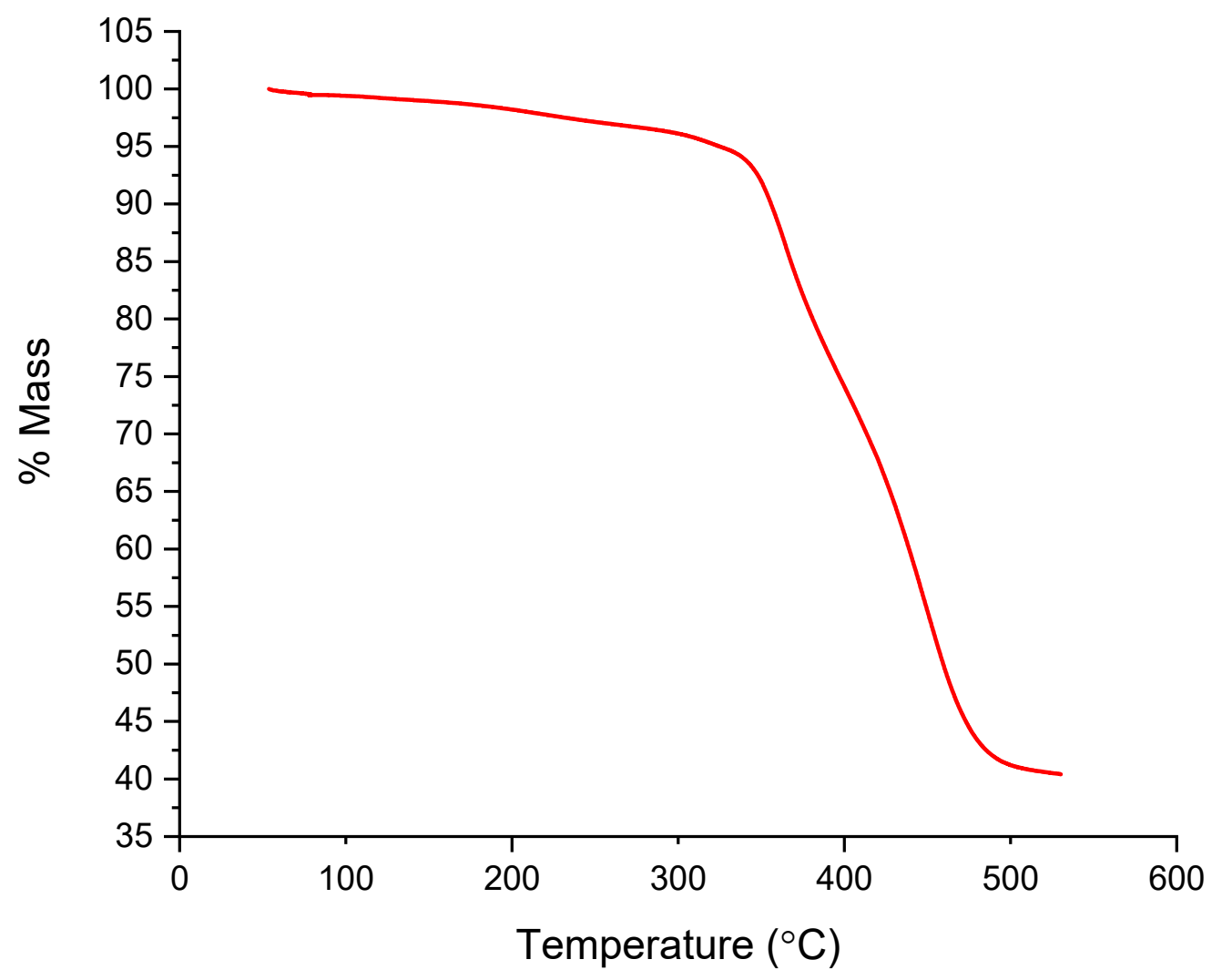

Figure S29. Thermogravimetric analysis of 10 at $20^{\circ} \mathrm{C}$ per minute. 
10. Emission Spectra

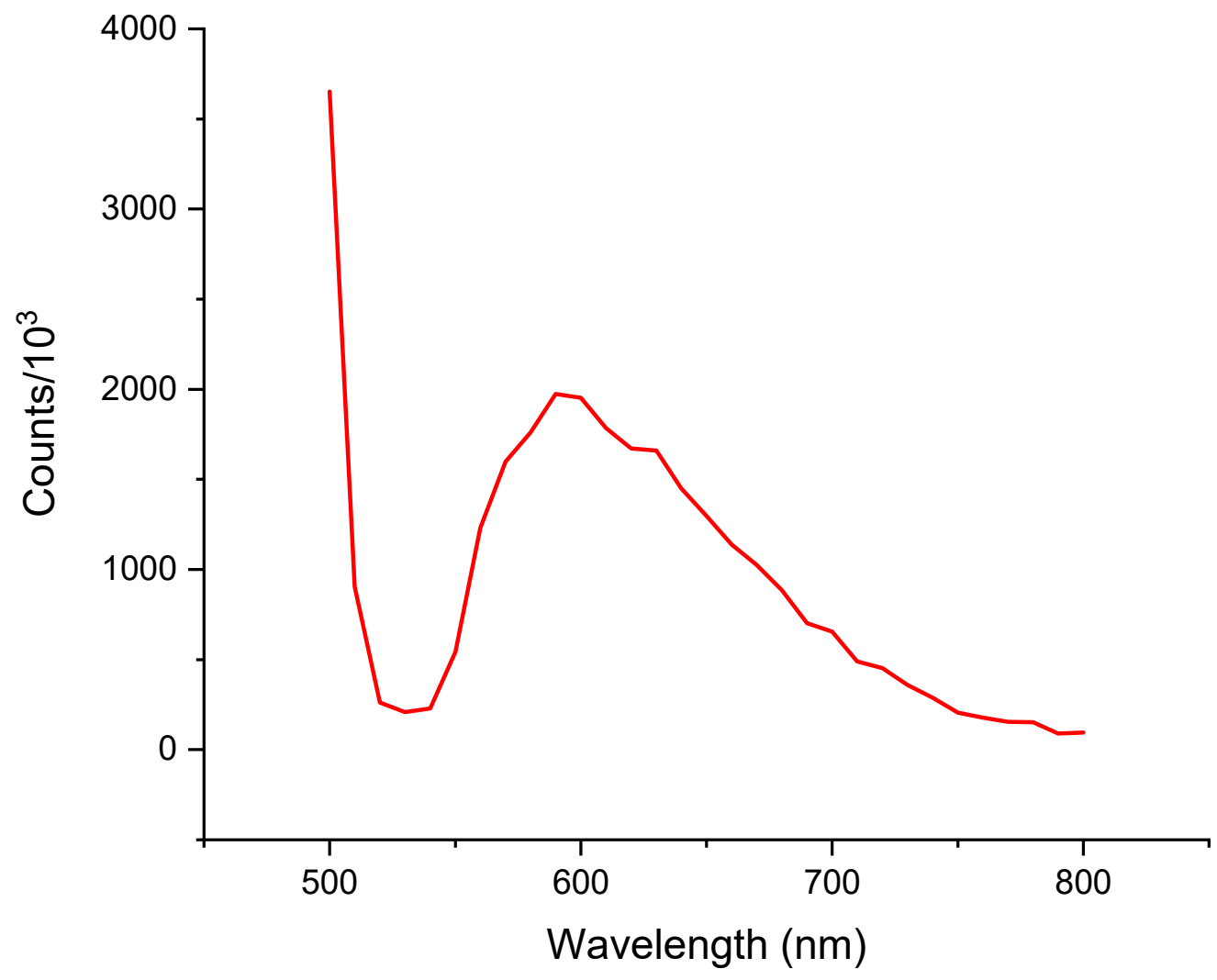

Figure S30. Emission spectrum of $\mathbf{3}(0.046 \mathrm{mM})$ at $503 \mathrm{~nm}$ in $\mathrm{C}_{6} \mathrm{H}_{5} \mathrm{Cl}$. 


\section{NMR Spectra}

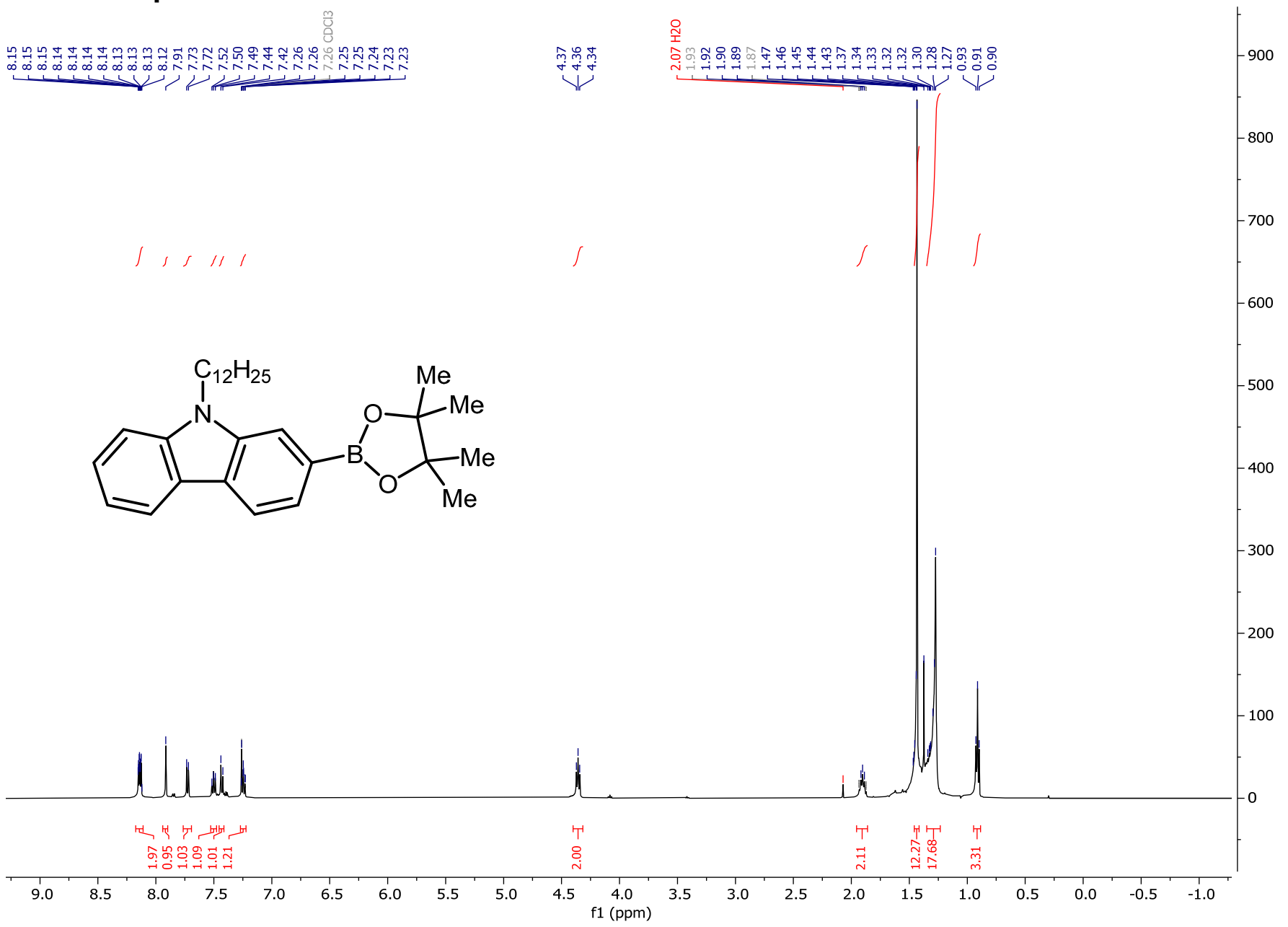

Figure S31. ${ }^{1} \mathrm{H}$ NMR spectrum of $\mathbf{S} \mathbf{1}\left(500 \mathrm{MHz}, \mathrm{CDCl}_{3}\right)$. 


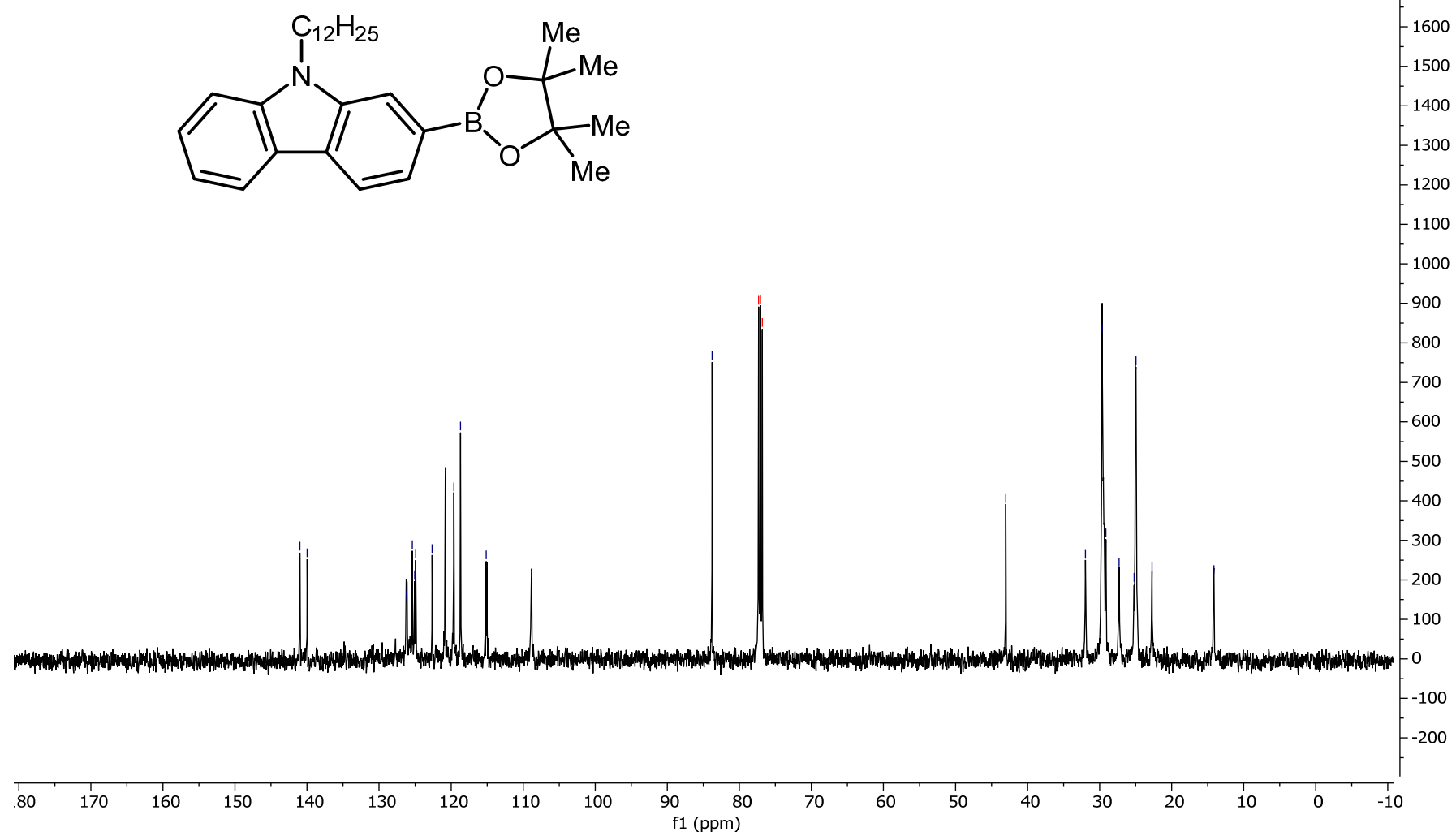

Figure S32. ${ }^{13} \mathrm{C}\left\{{ }^{1} \mathrm{H}\right\}$ NMR spectrum of $\mathbf{S 1}\left(126 \mathrm{MHz}, \mathrm{CDCl}_{3}\right)$. 


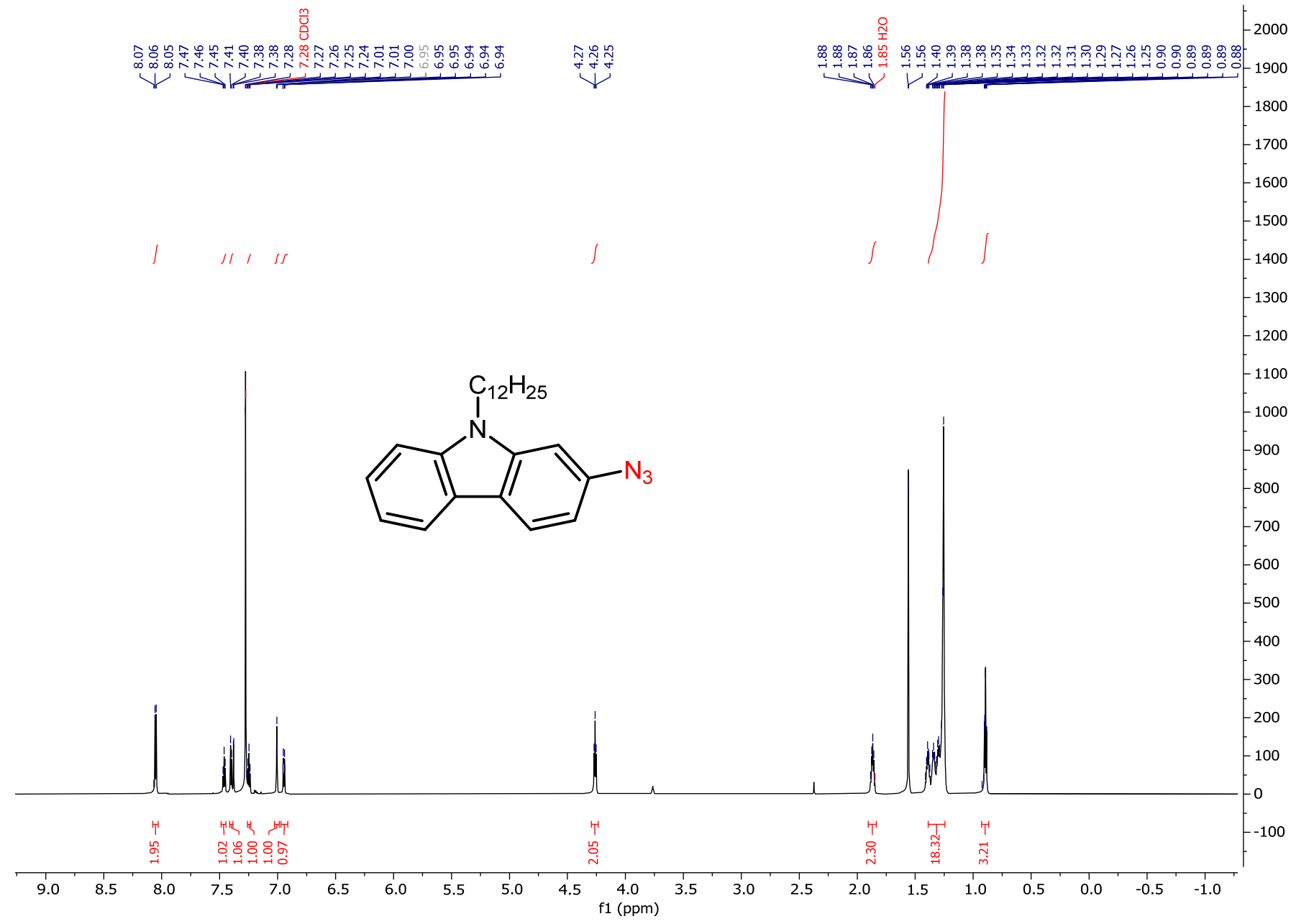

Figure S33. ${ }^{1} \mathrm{H}$ NMR spectrum of $\mathbf{4 b}\left(800 \mathrm{MHz}, \mathrm{CDCl}_{3}\right)$. 


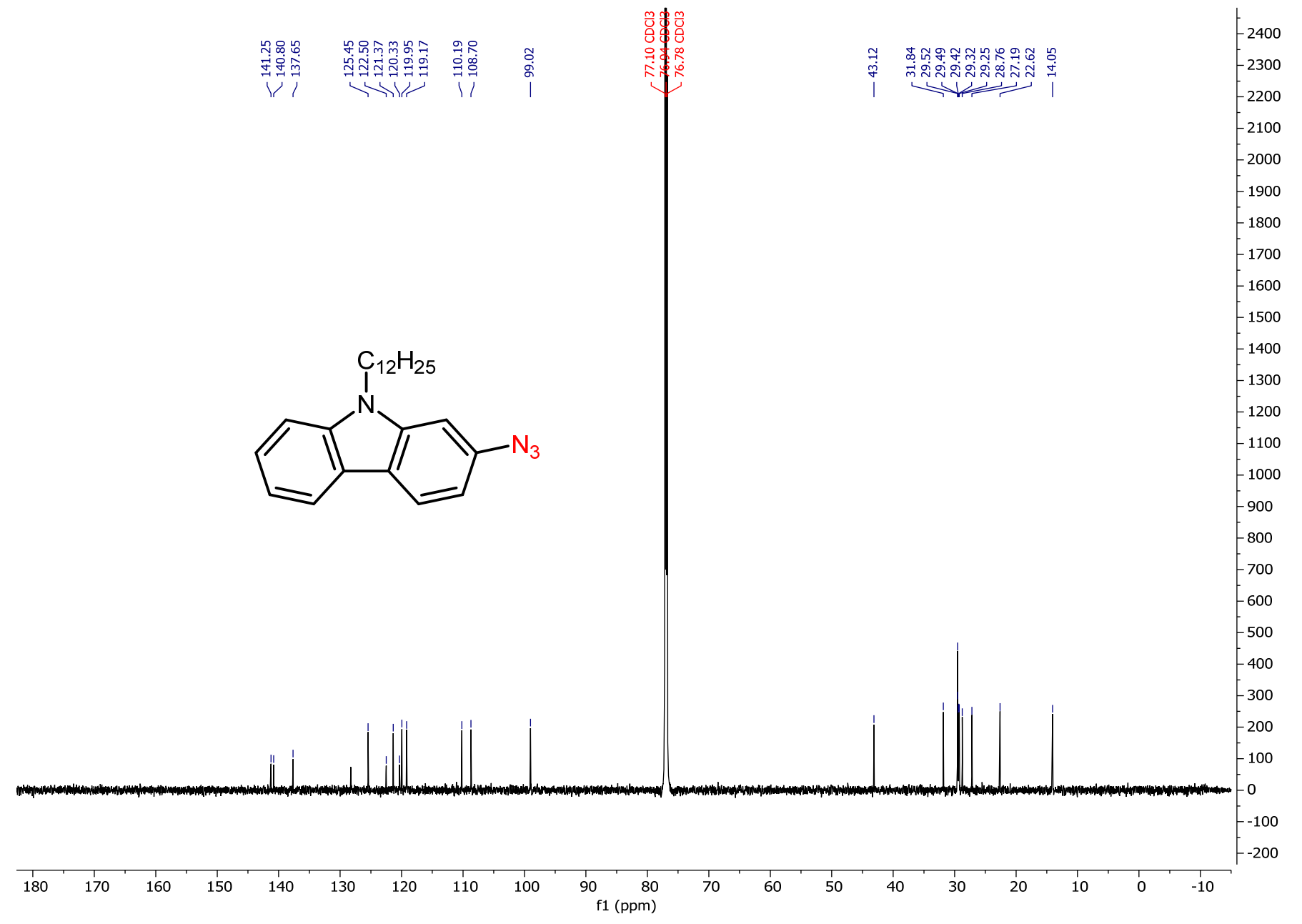

Figure S34. ${ }^{13} \mathrm{C}\left\{{ }^{1} \mathrm{H}\right\}$ NMR spectrum of $\mathbf{4 b}\left(201 \mathrm{MHz}, \mathrm{CDCl}_{3}\right)$. 


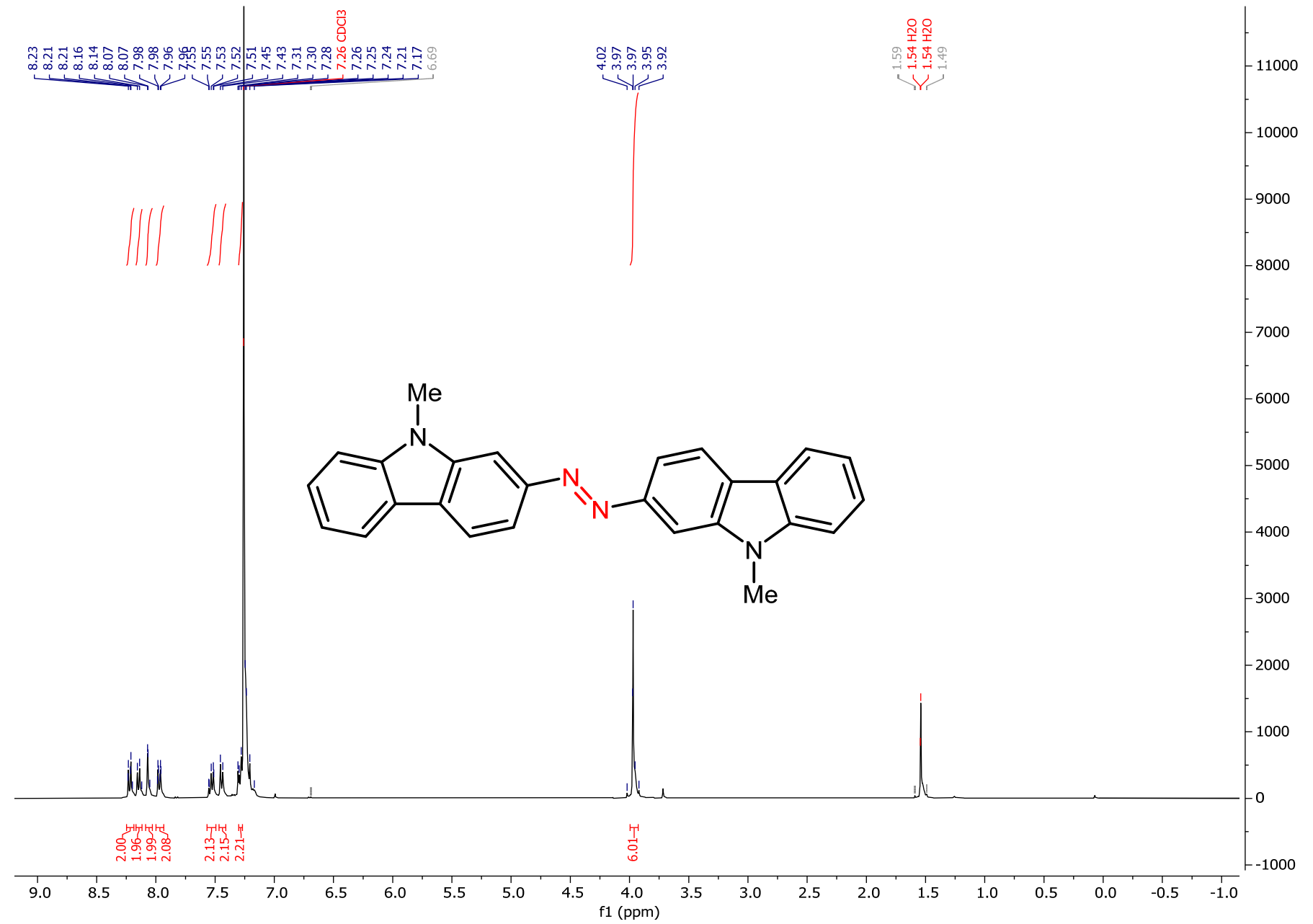

Figure S35. ${ }^{1} \mathrm{H}$ NMR spectrum of $\mathbf{5 a}\left(400 \mathrm{MHz}^{\mathrm{CDCl}} 3\right)$. 


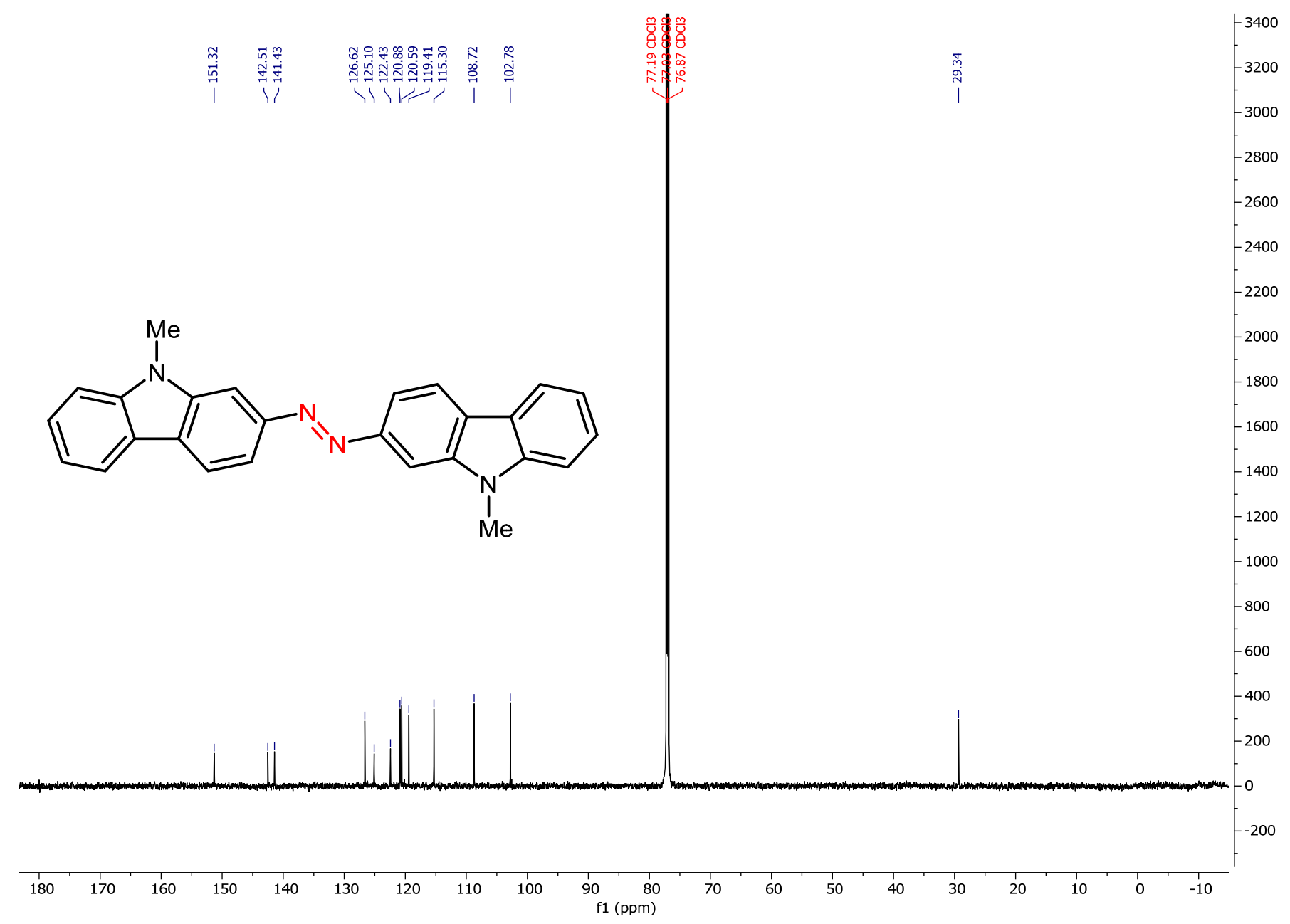

Figure S36. ${ }^{13} \mathrm{C}\left\{{ }^{1} \mathrm{H}\right\}$ NMR spectrum of $\mathbf{5 a}\left(201 \mathrm{MHz}, \mathrm{CDCl}_{3}\right)$. 


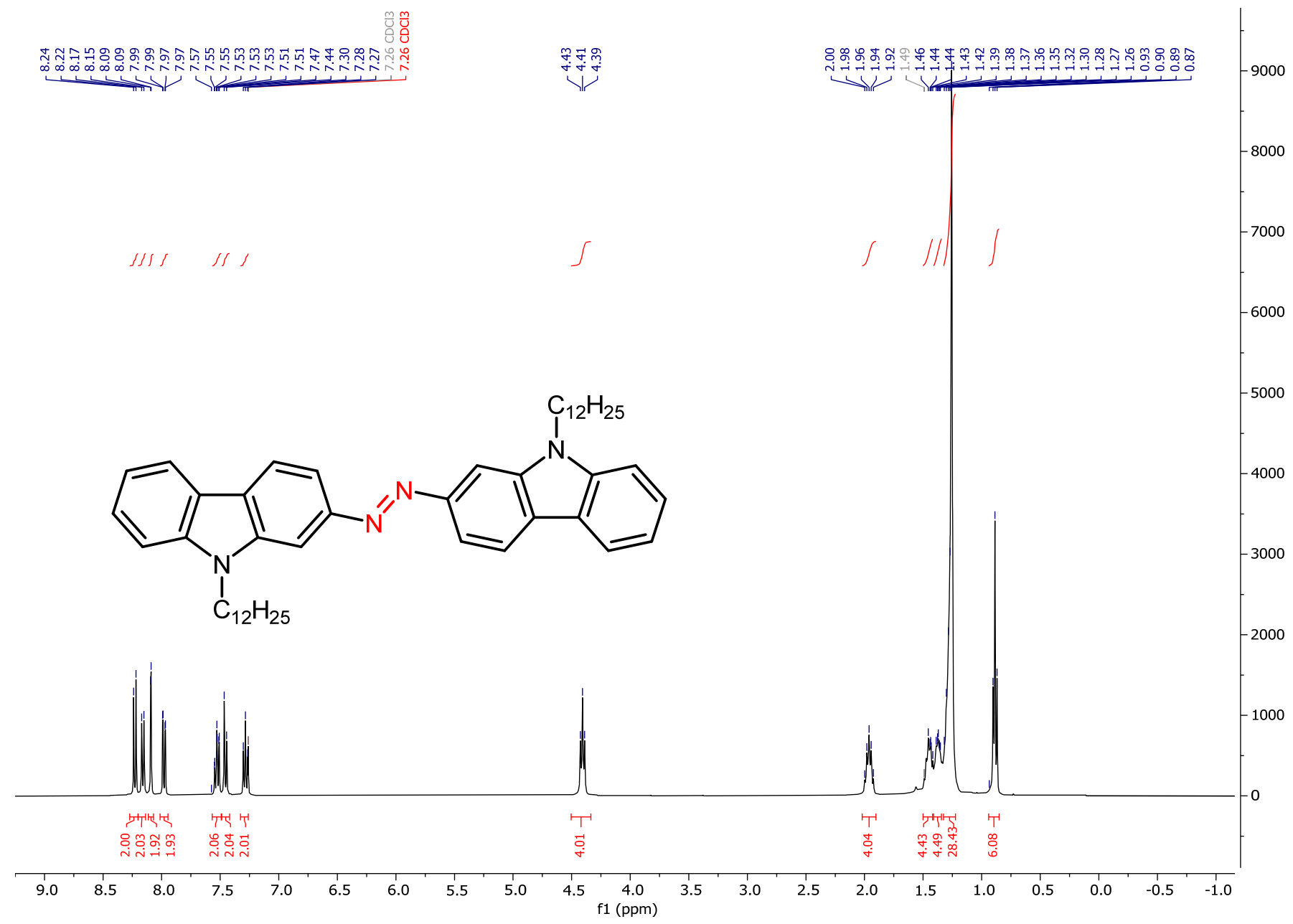

Figure S37. ${ }^{1} \mathrm{H}$ NMR spectrum of $\mathbf{5 b}\left(400 \mathrm{MHz}, \mathrm{CDCl}_{3}\right)$. 

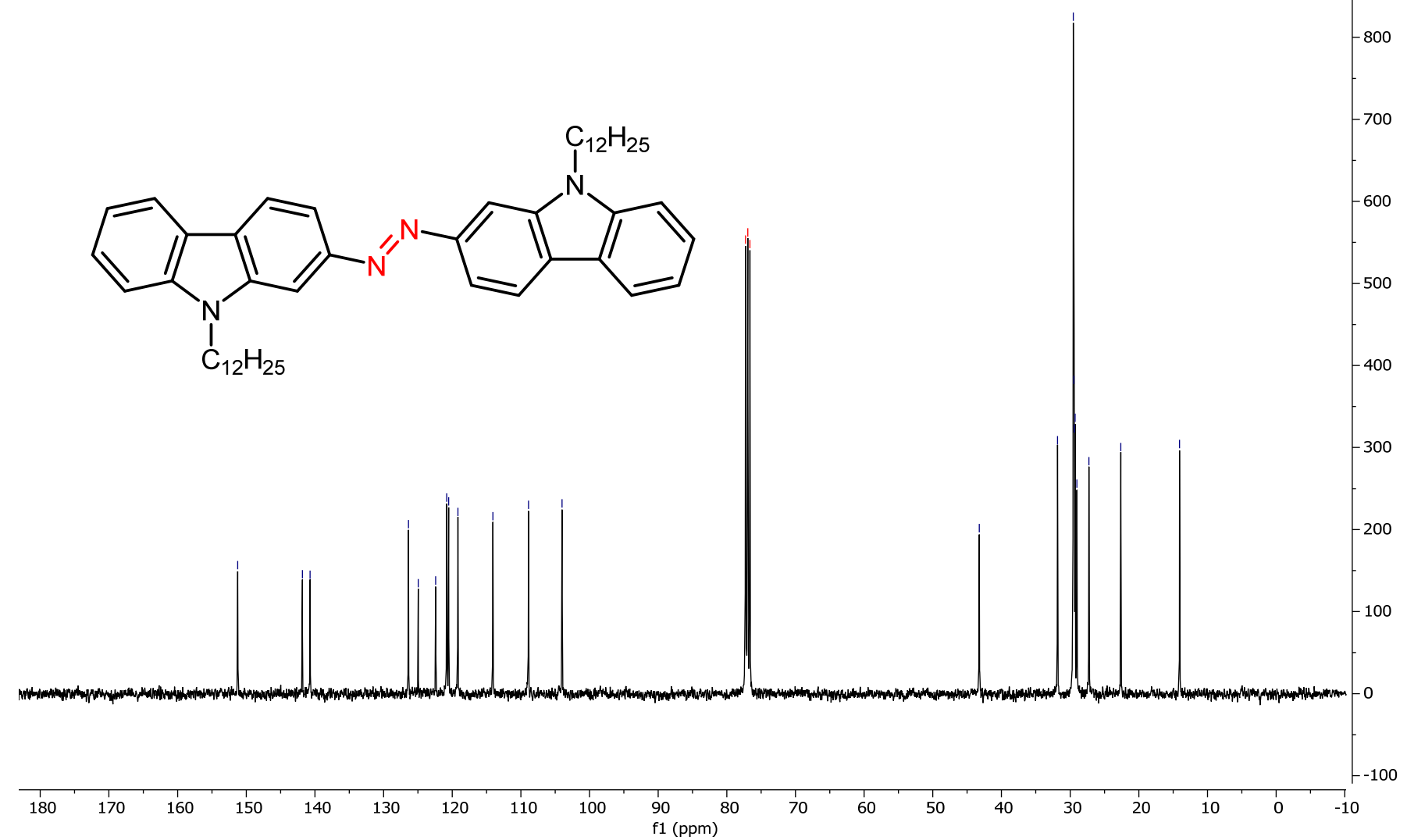

Figure S38. ${ }^{13} \mathrm{C}\left\{{ }^{1} \mathrm{H}\right\}$ NMR spectrum of $\mathbf{5 b}\left(126 \mathrm{MHz}, \mathrm{CDCl}_{3}\right)$. 


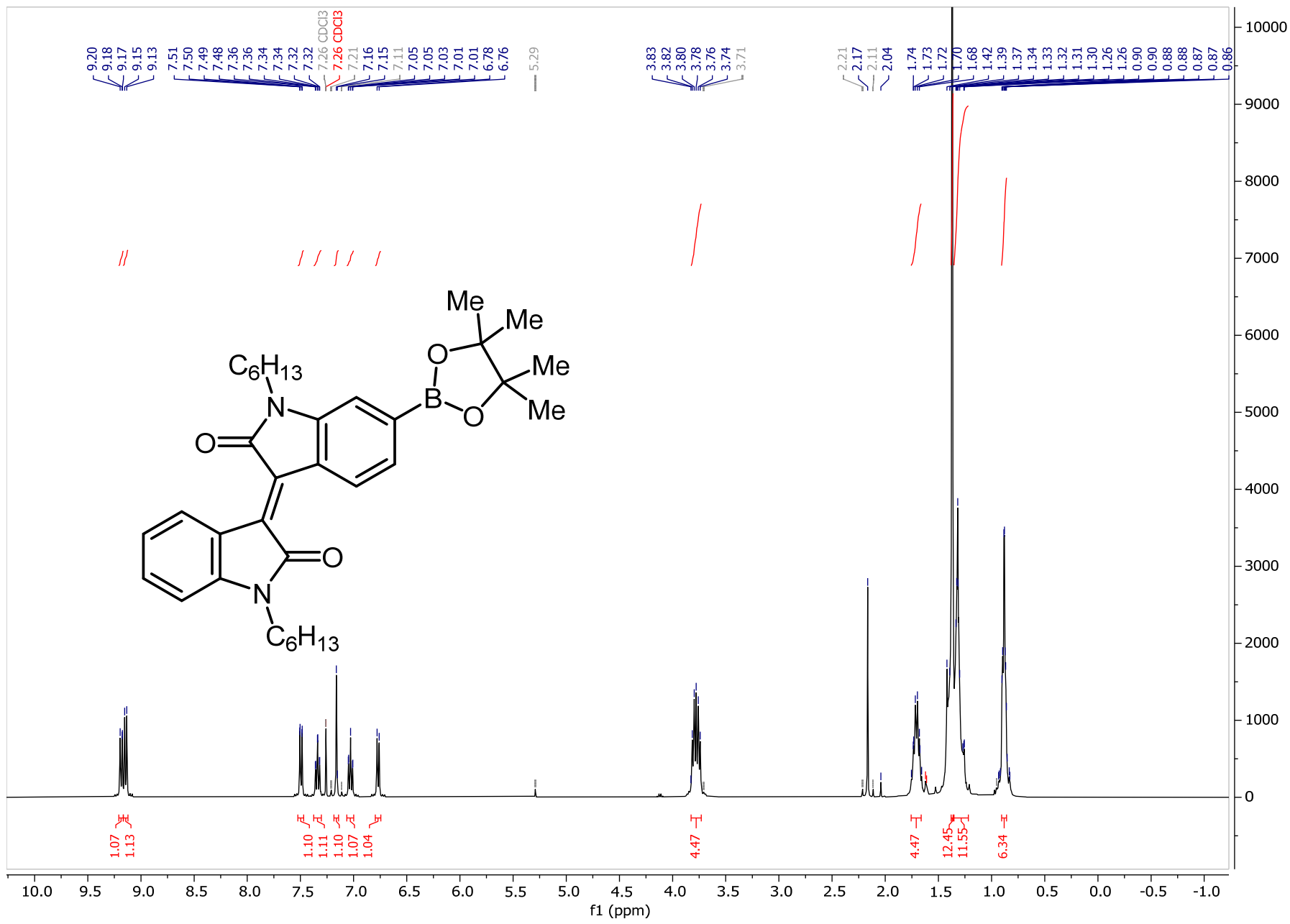

Figure S39. ${ }^{1} \mathrm{H}$ NMR spectrum of $\mathbf{S} 2\left(400 \mathrm{MHz}, \mathrm{CDCl}_{3}\right)$. 


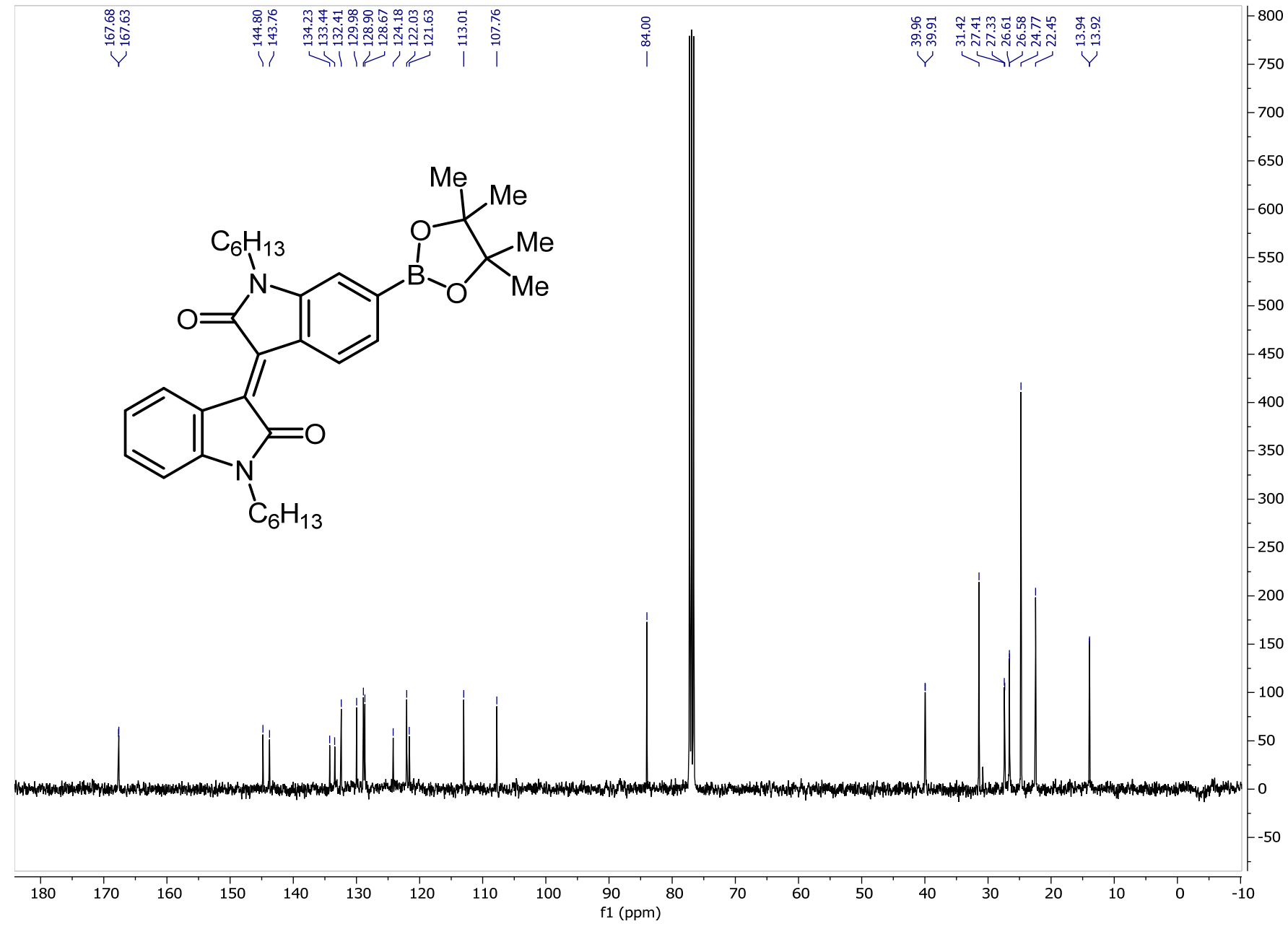

Figure S40. ${ }^{13} \mathrm{C}\left\{{ }^{1} \mathrm{H}\right\}$ NMR spectrum of $\mathbf{S} 2\left(101 \mathrm{MHz}, \mathrm{CDCl}_{3}\right)$. 


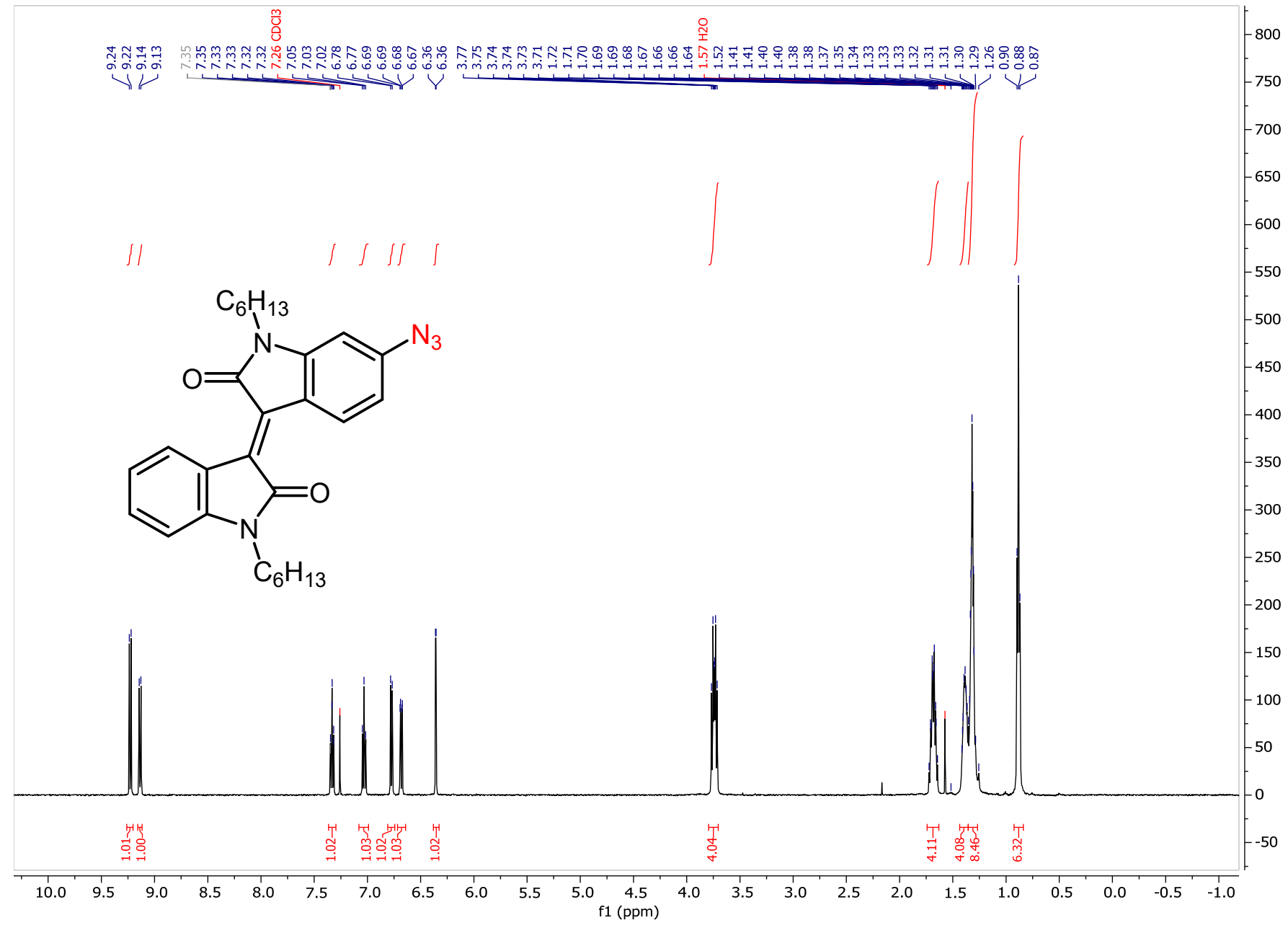

Figure $\mathbf{S 4 1} .{ }^{1} \mathrm{H}$ NMR spectrum of $\mathbf{S} 3\left(500 \mathrm{MHz}, \mathrm{CDCl}_{3}\right)$. 


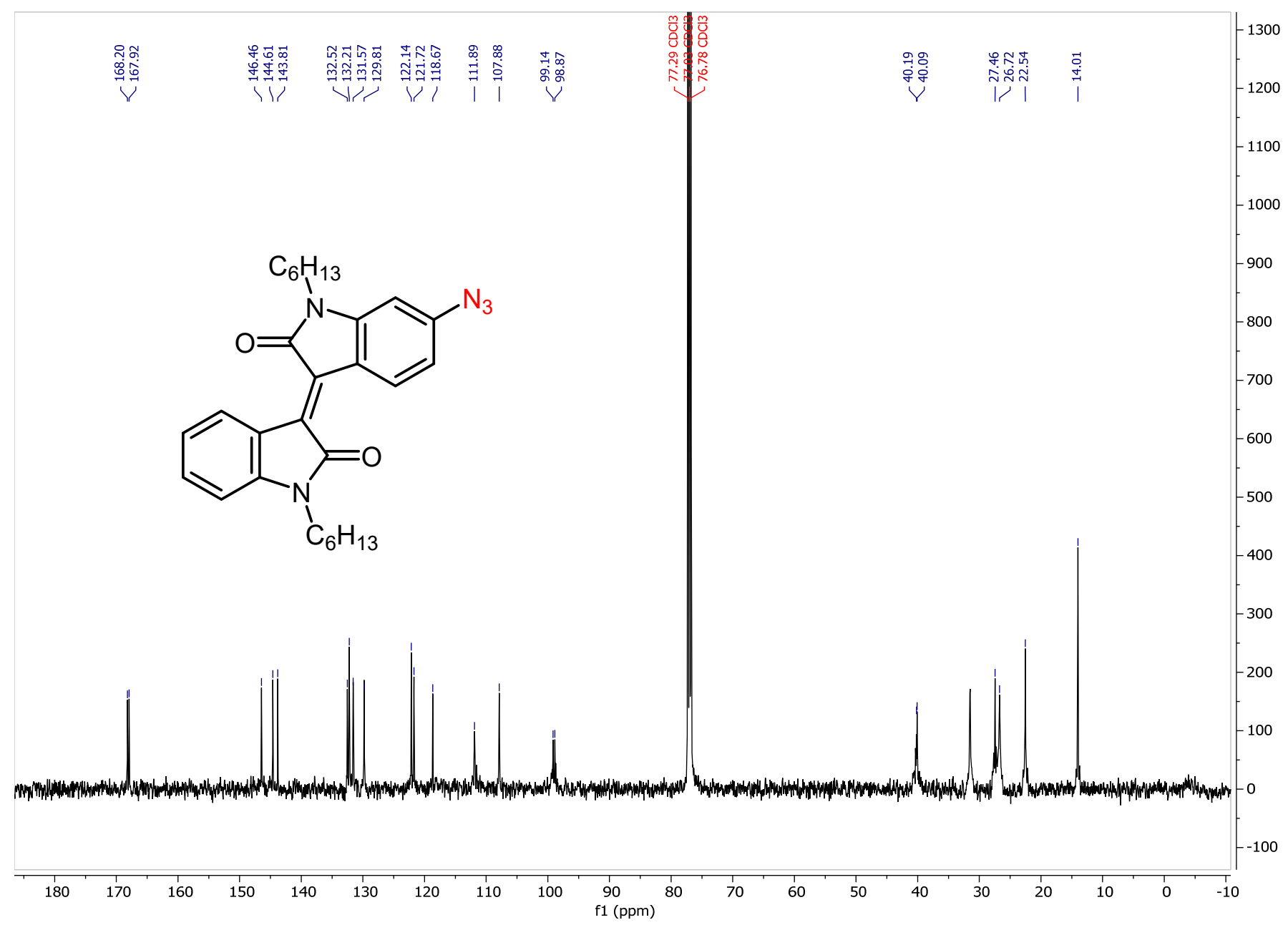

Figure S42. ${ }^{13} \mathrm{C}\left\{{ }^{1} \mathrm{H}\right\}$ NMR spectrum of $\mathbf{S 3}\left(126 \mathrm{MHz}, \mathrm{CDCl}_{3}\right)$. 

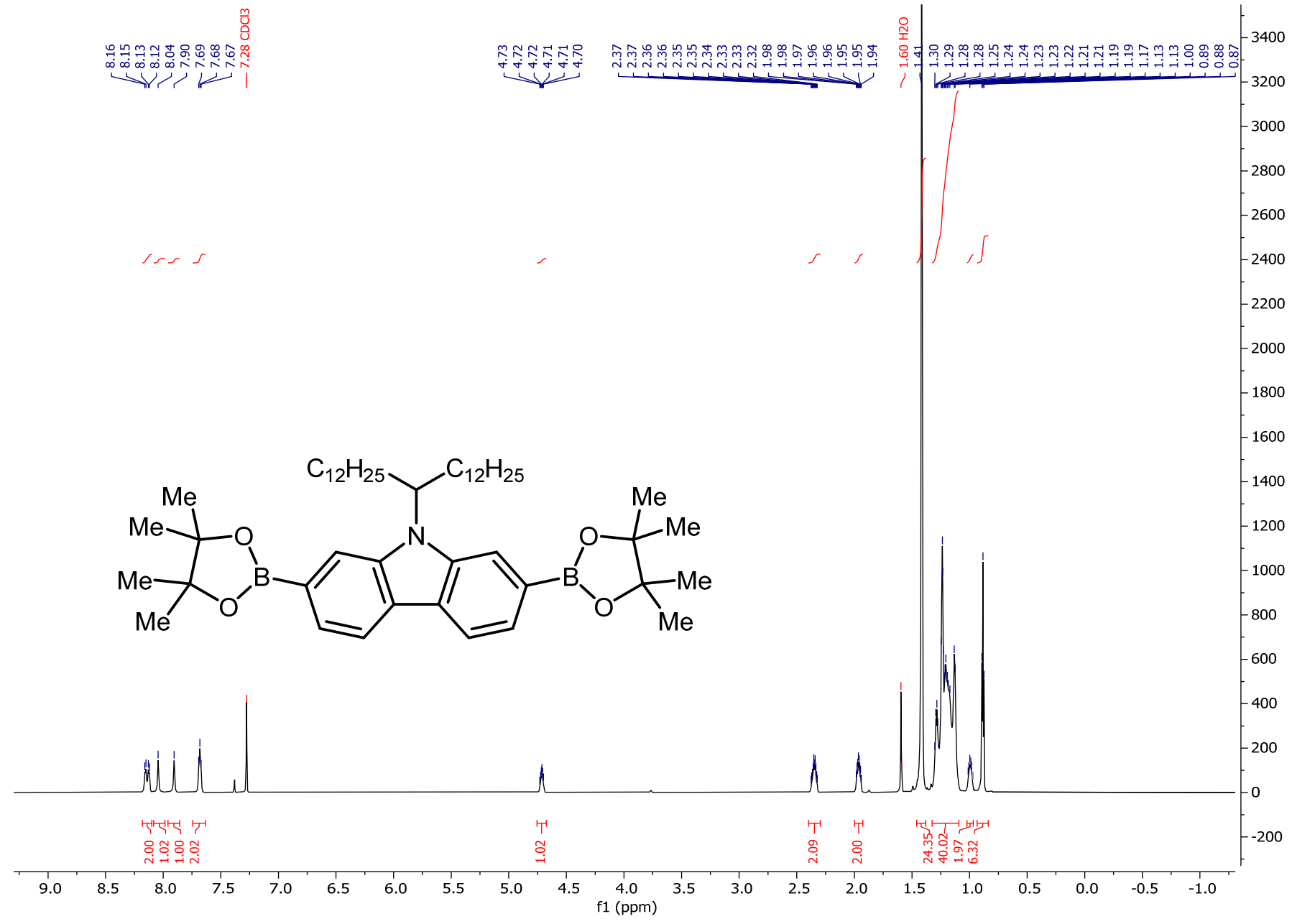

Figure S43. ${ }^{1} \mathrm{H}$ NMR spectrum of $\mathbf{S 4}\left(800 \mathrm{MHz}, \mathrm{CDCl}_{3}\right)$. 


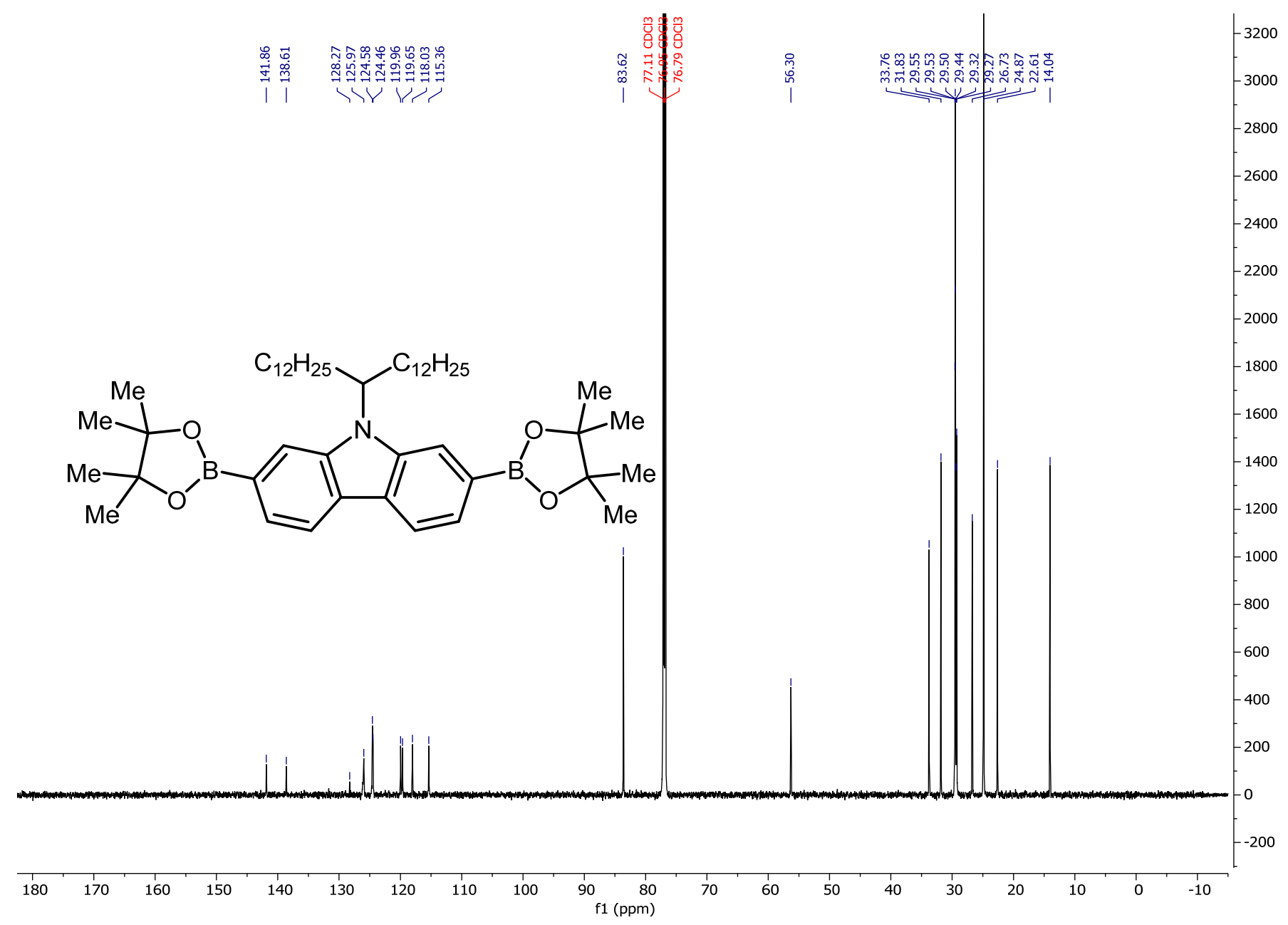

Figure S44. ${ }^{13} \mathrm{C}\left\{{ }^{1} \mathrm{H}\right\}$ NMR spectrum of $\mathbf{S 4}\left(201 \mathrm{MHz}, \mathrm{CDCl}_{3}\right)$. 


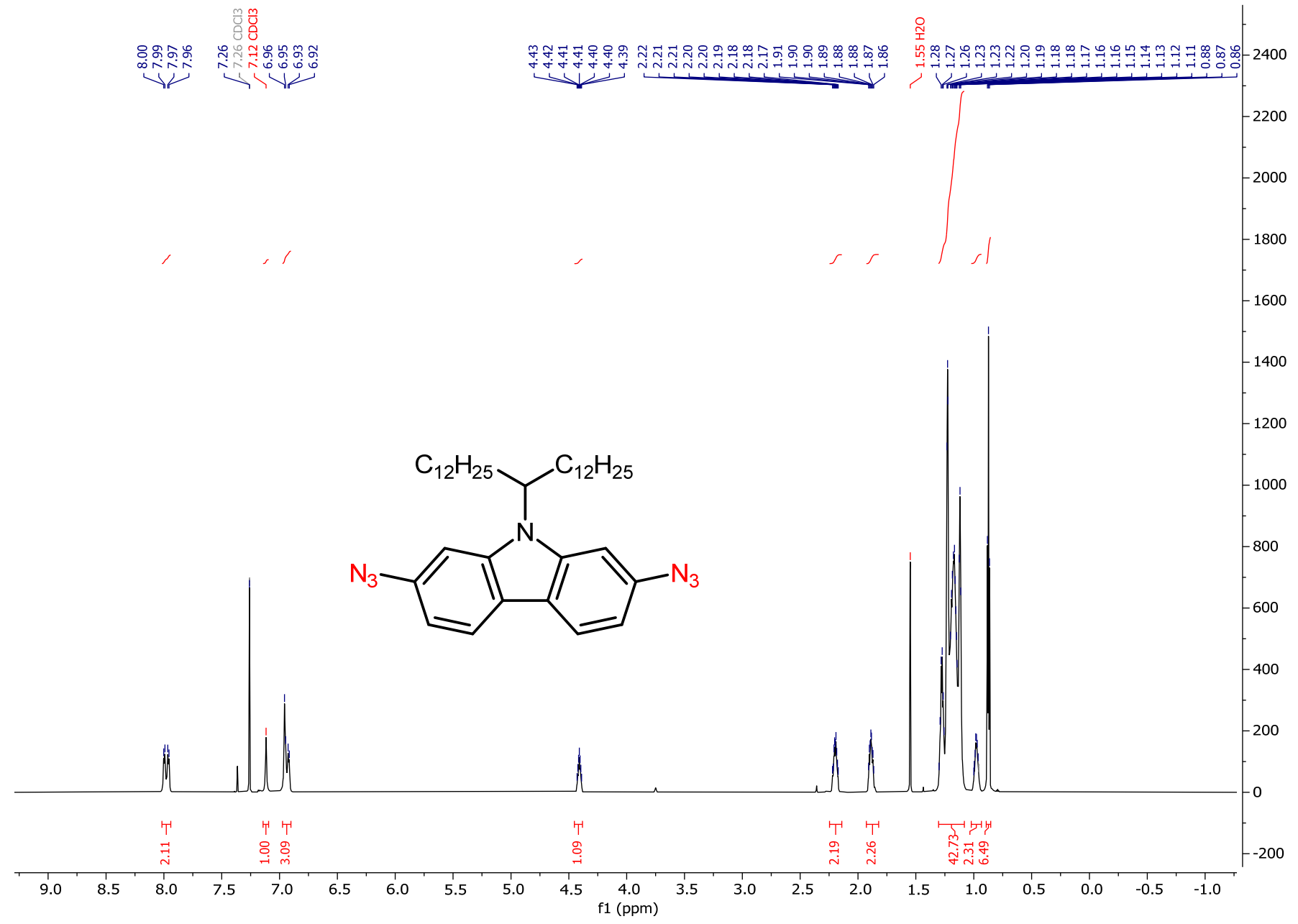

Figure S45. ${ }^{1} \mathrm{H}$ NMR spectrum of $2\left(800 \mathrm{MHz}^{\mathrm{C}} \mathrm{CDCl}_{3}\right)$. 


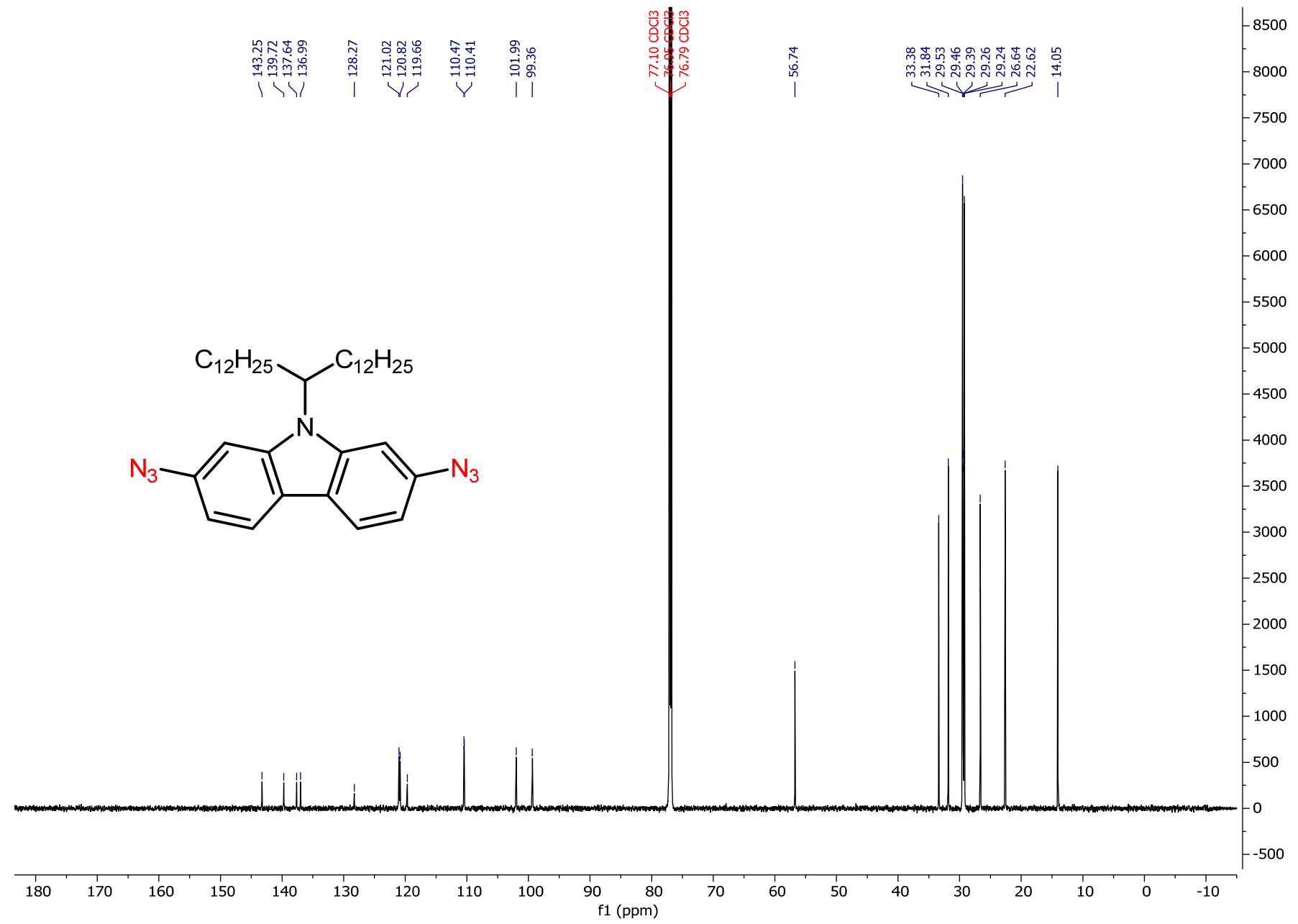

Figure S46. ${ }^{13} \mathrm{C}\left\{{ }^{1} \mathrm{H}\right\} \mathrm{NMR}$ spectrum of $2\left(201 \mathrm{MHz}, \mathrm{CDCl}_{3}\right)$. 


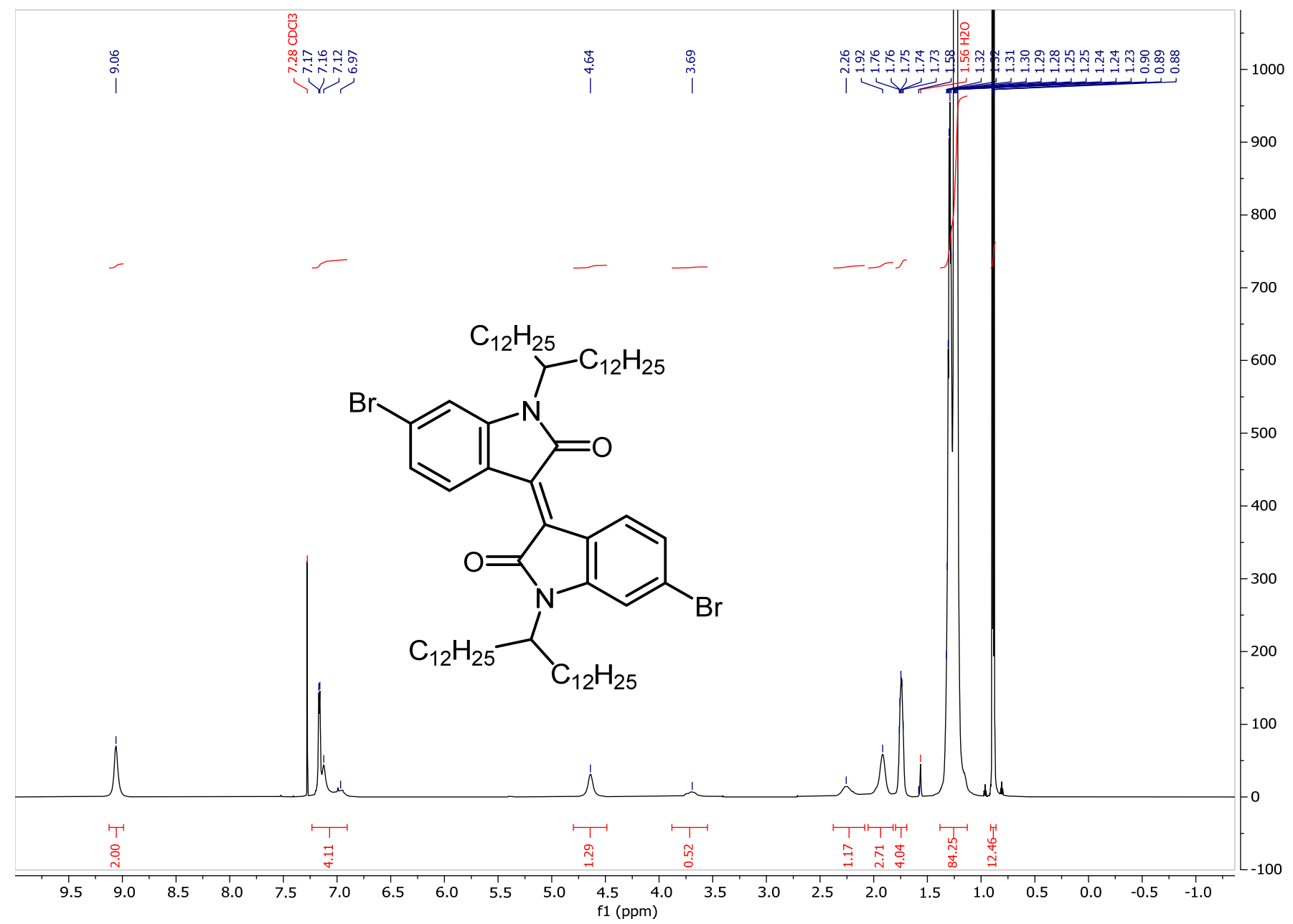

Figure S47. ${ }^{1} \mathrm{H}$ NMR spectrum of $\mathbf{S 5}\left(300 \mathrm{MHz}, \mathrm{CDCl}_{3}\right)$. 


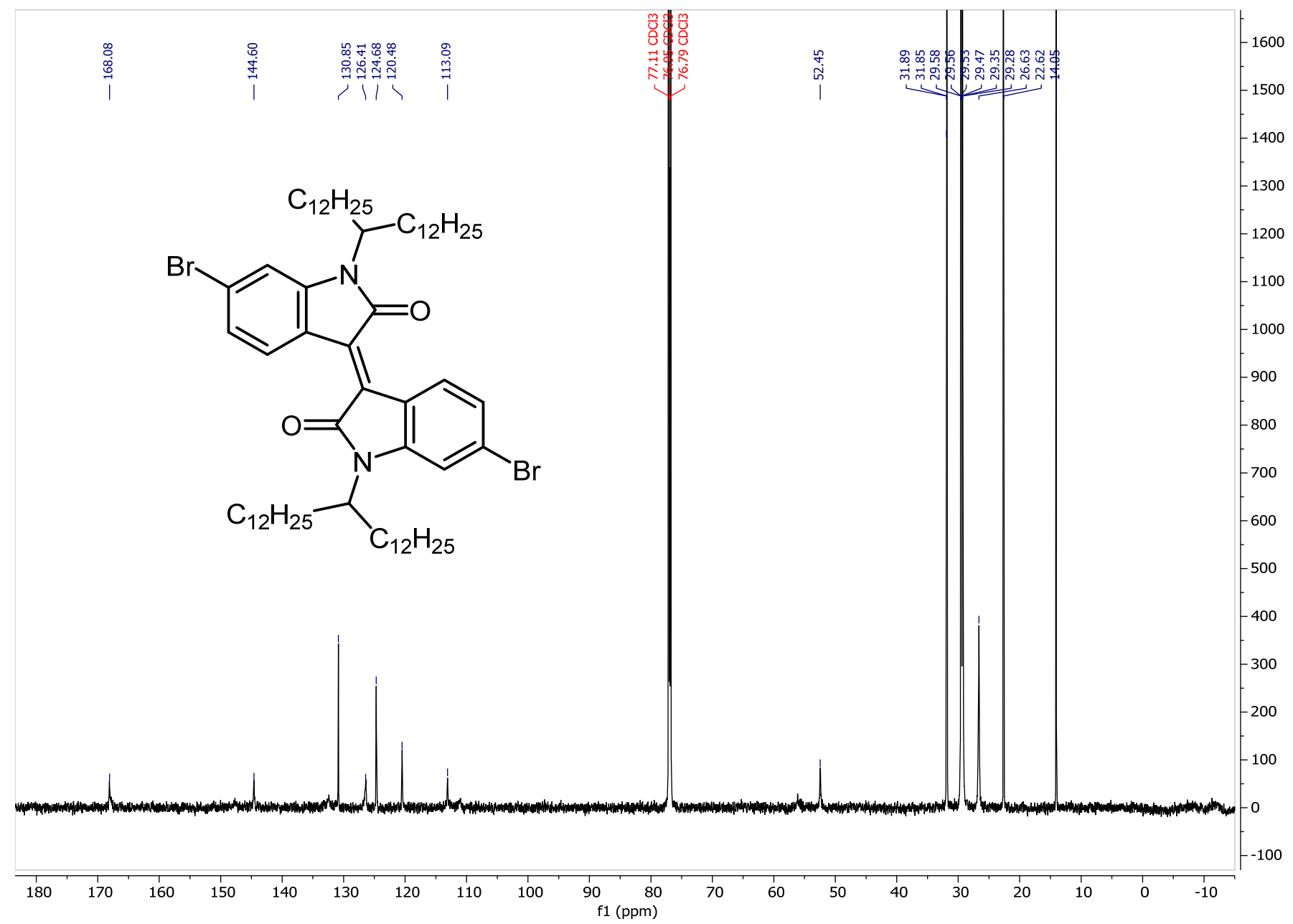

Figure S48. ${ }^{13} \mathrm{C}\left\{{ }^{1} \mathrm{H}\right\}$ NMR spectrum of $\mathbf{S 5}\left(201 \mathrm{MHz}, \mathrm{CDCl}_{3}\right)$. 


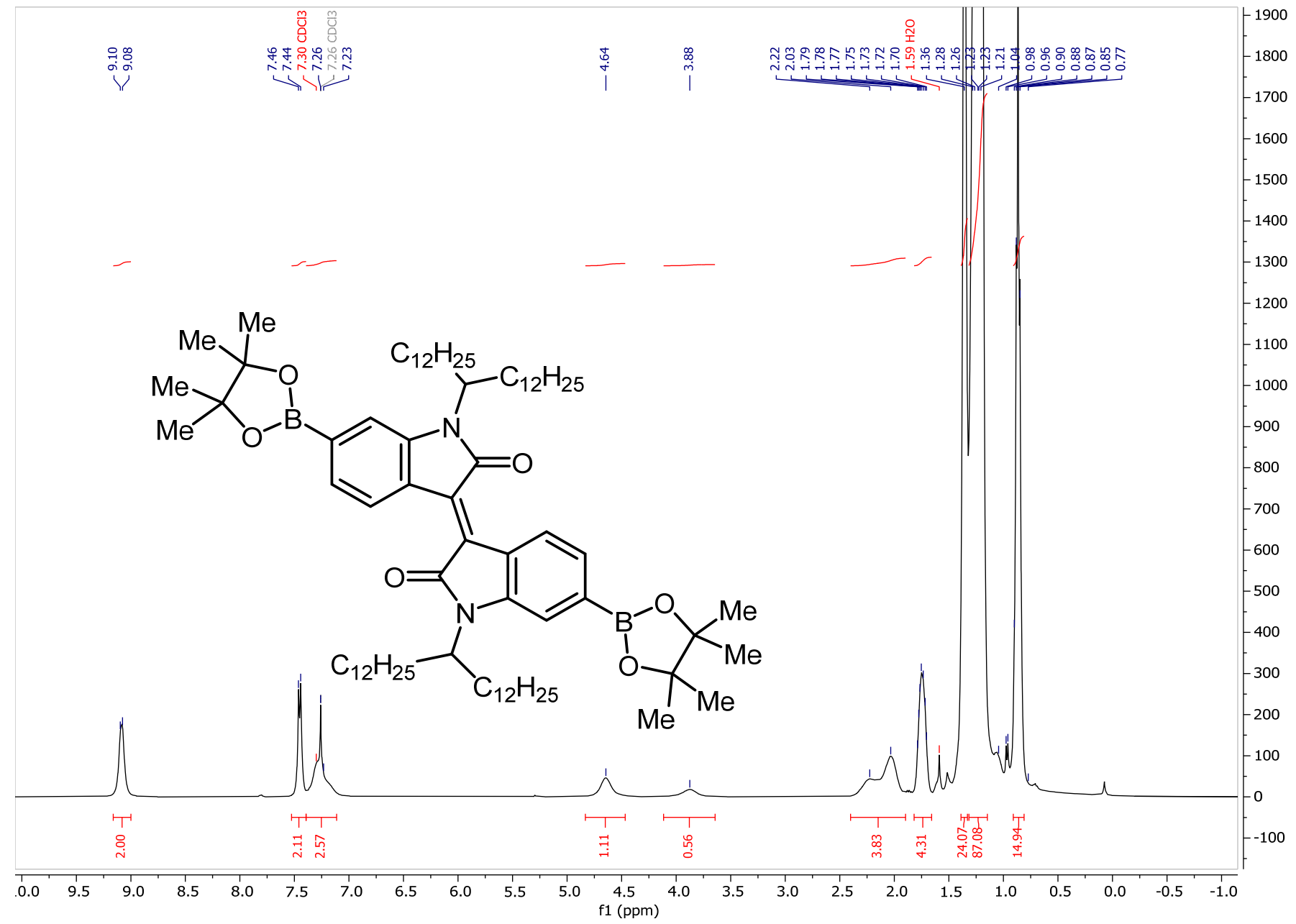

Figure S49. ${ }^{1} \mathrm{H}$ NMR spectrum of $\mathbf{S} \mathbf{6}\left(400 \mathrm{MHz}, \mathrm{CDCl}_{3}\right)$. 


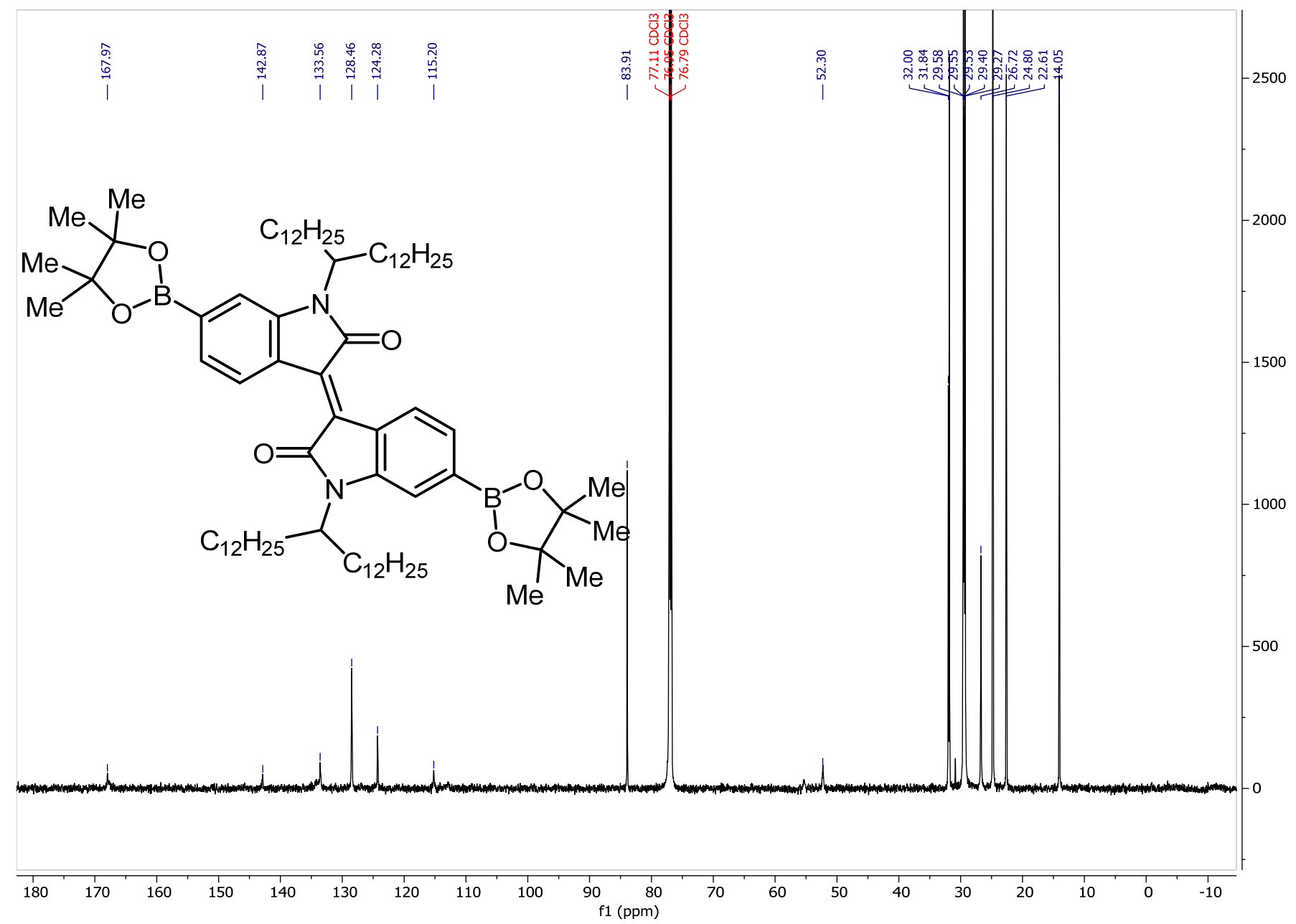

Figure S50. ${ }^{13} \mathrm{C}\left\{{ }^{1} \mathrm{H}\right\}$ NMR spectrum of $\mathbf{S 6}\left(201 \mathrm{MHz}, \mathrm{CDCl}_{3}\right)$. 


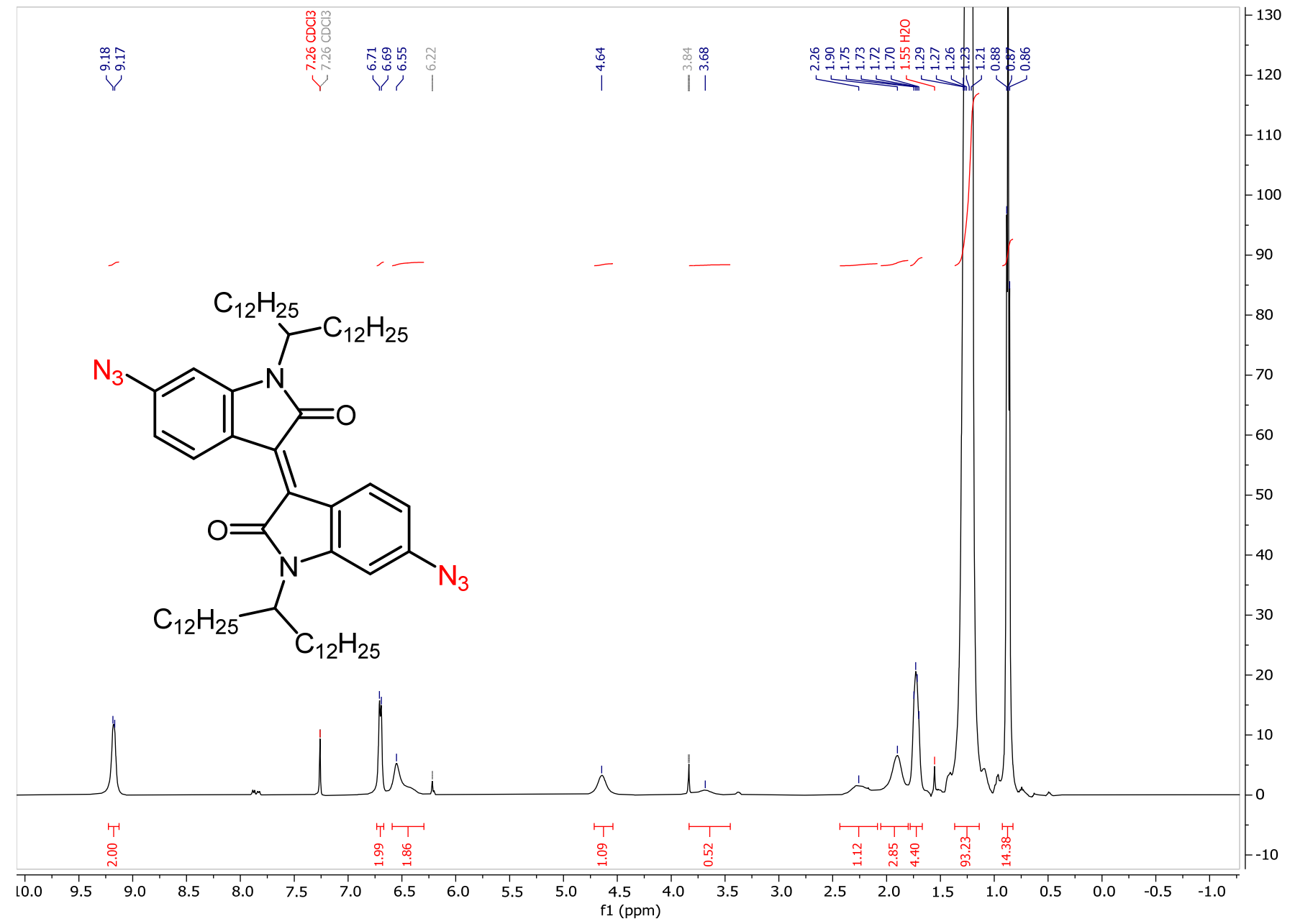

Figure S51. ${ }^{1} \mathrm{H}$ NMR spectrum of $\mathbf{S 7}\left(500 \mathrm{MHz}^{\mathrm{CDCl}} 3\right)$. 


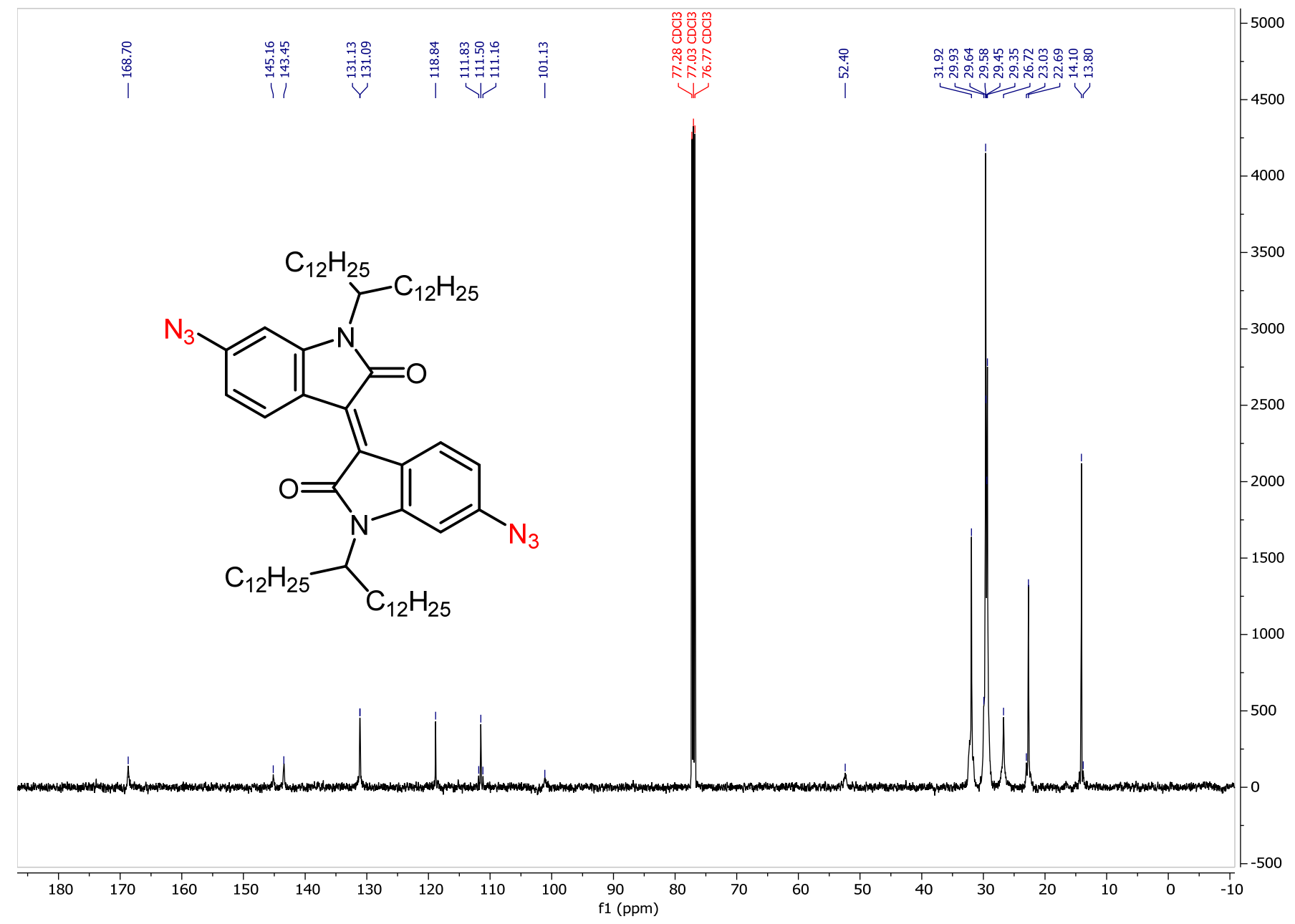

Figure S52. ${ }^{13} \mathrm{C}\left\{{ }^{1} \mathrm{H}\right\}$ NMR spectrum of $\mathbf{S 7}\left(126 \mathrm{MHz}, \mathrm{CDCl}_{3}\right)$. 


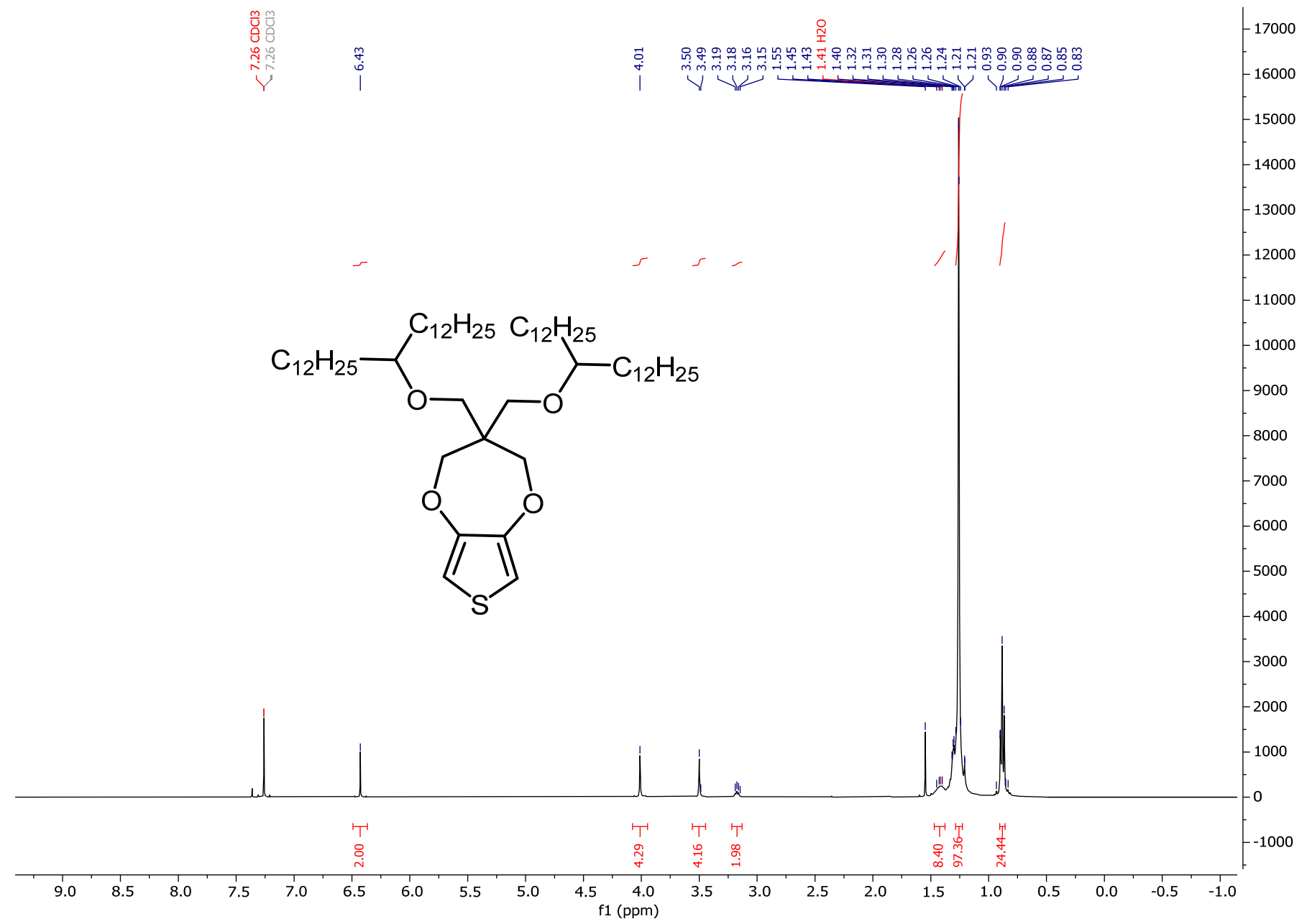

Figure S53. ${ }^{1} \mathrm{H}$ NMR spectrum of $\mathbf{S 8}\left(400 \mathrm{MHz}_{2} \mathrm{CDCl}_{3}\right)$. 


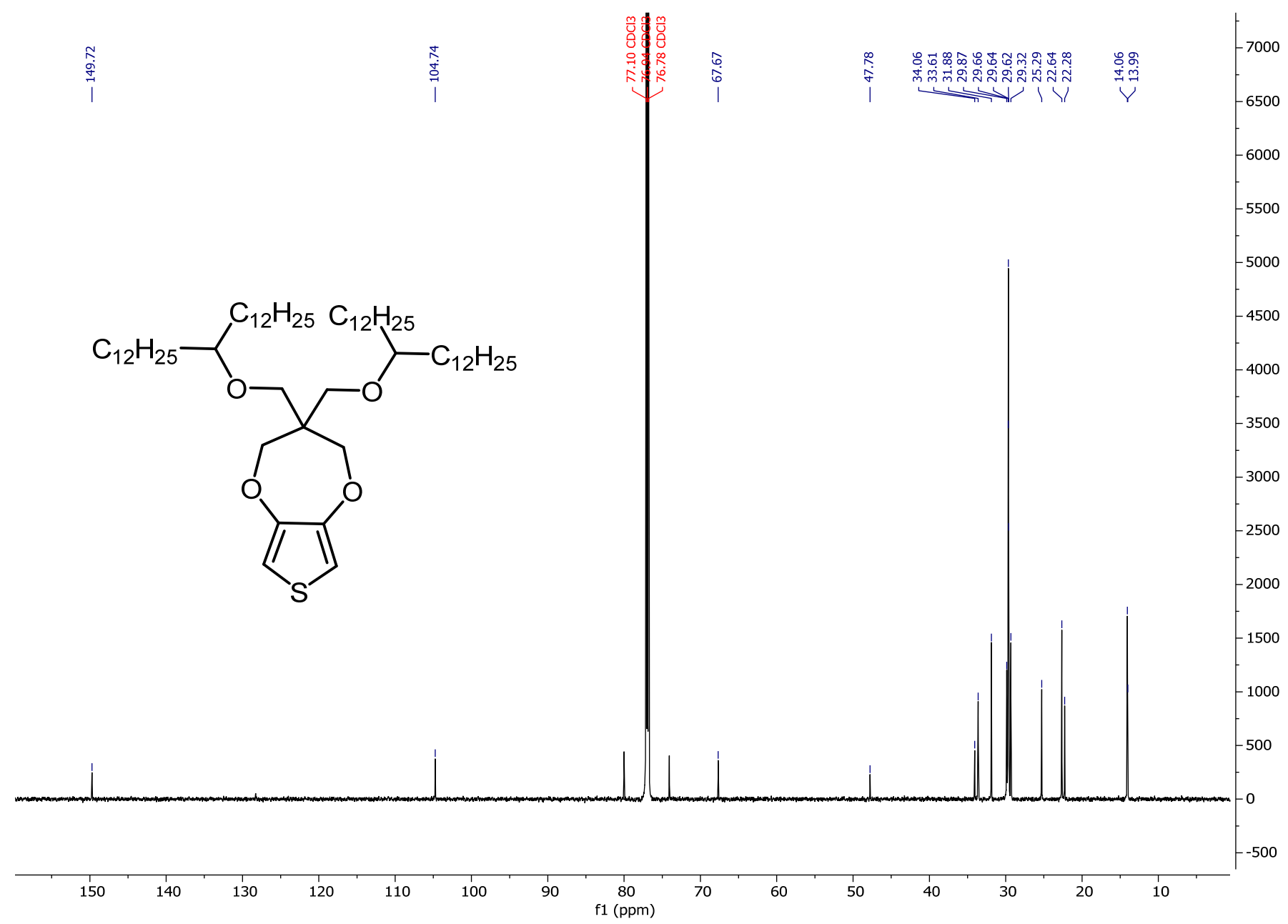

Figure S54. ${ }^{13} \mathrm{C}\left\{{ }^{1} \mathrm{H}\right\}$ NMR spectrum of $\mathbf{S 8}\left(201 \mathrm{MHz}, \mathrm{CDCl}_{3}\right)$. 


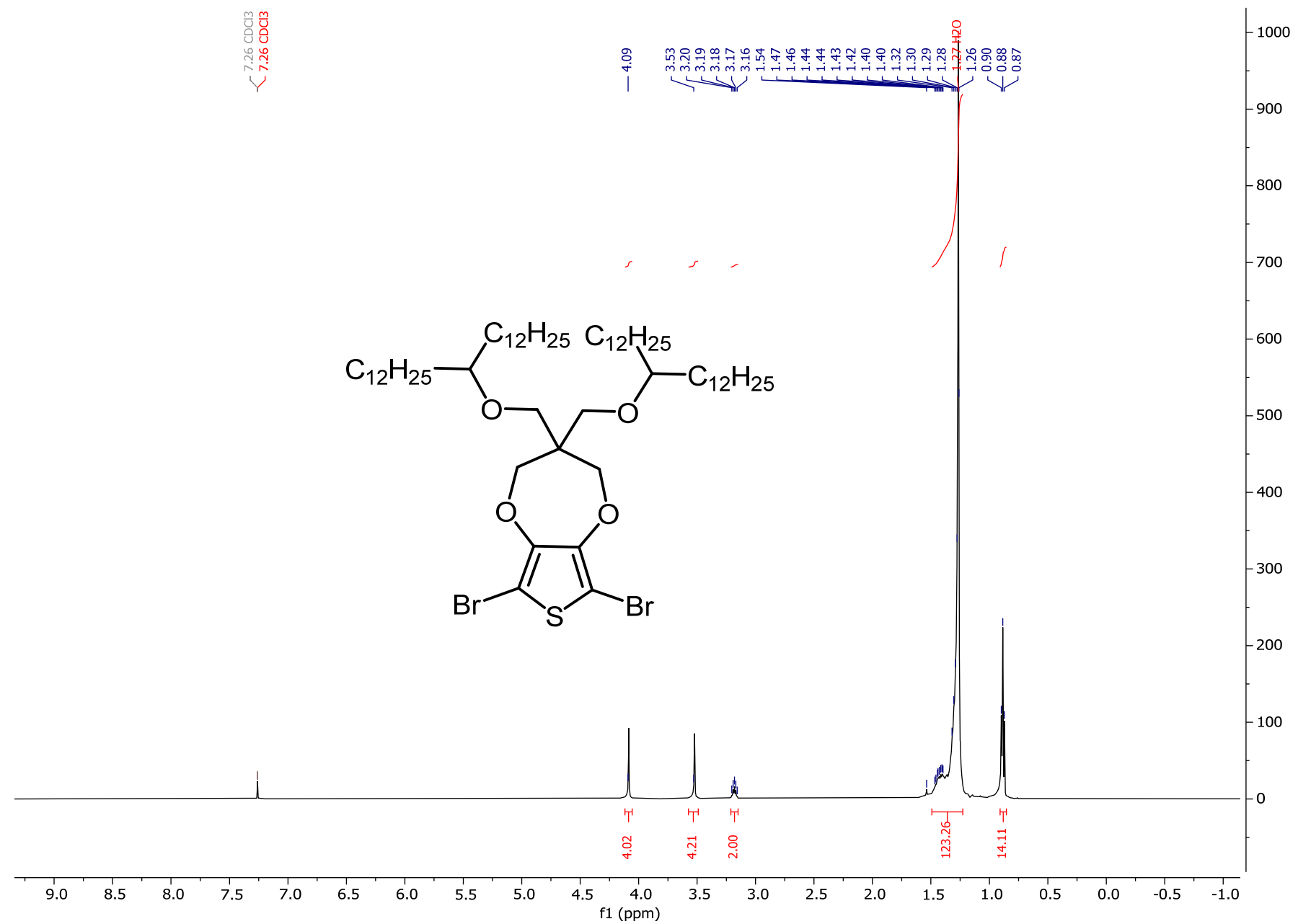

Figure S55. ${ }^{1} \mathrm{H}$ NMR spectrum of $\mathbf{S} 9\left(400 \mathrm{MHz}, \mathrm{CDCl}_{3}\right)$. 


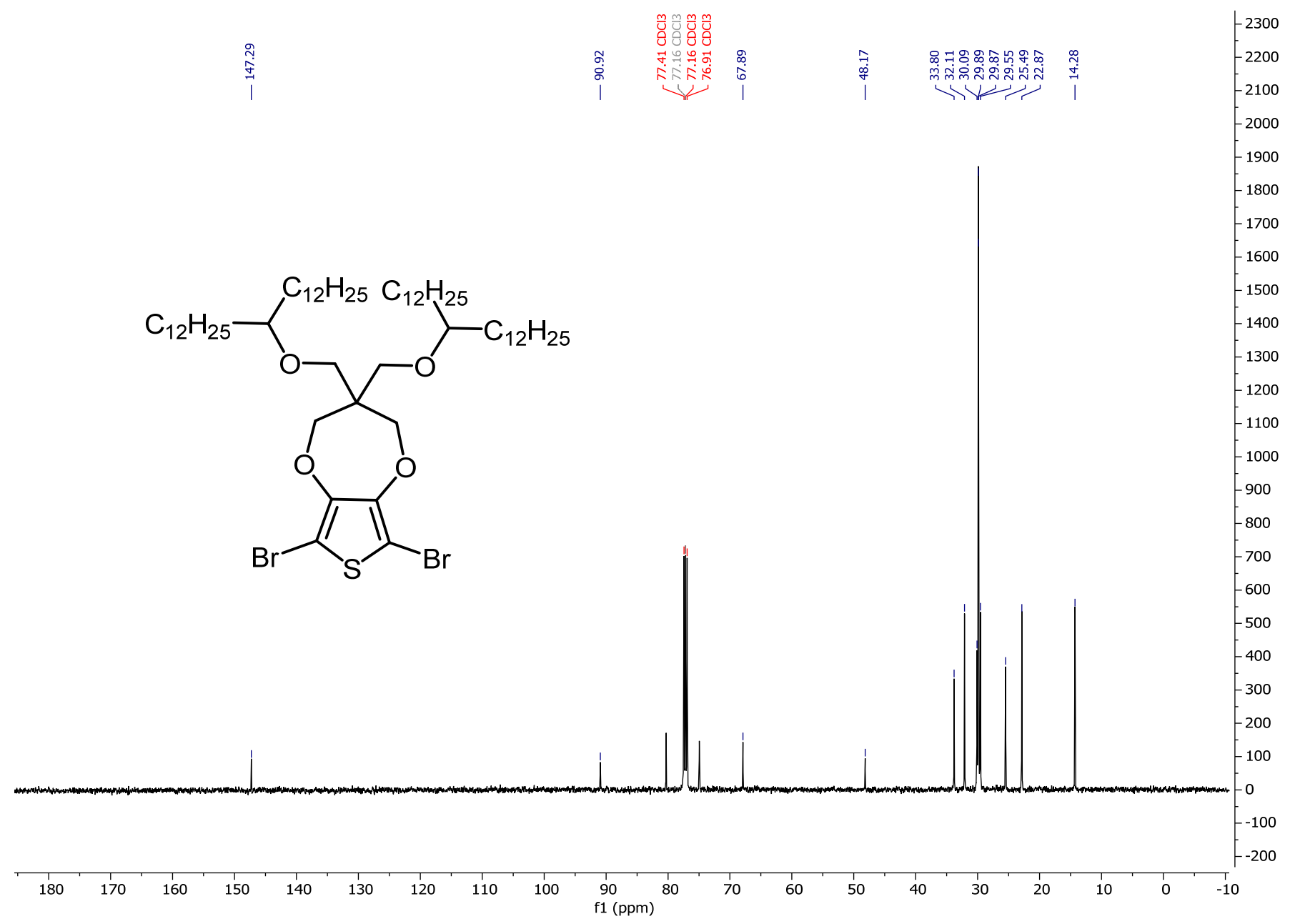

Figure S56. ${ }^{13} \mathrm{C}\left\{{ }^{1} \mathrm{H}\right\}$ NMR spectrum of $\mathbf{S 9}\left(101 \mathrm{MHz}, \mathrm{CDCl}_{3}\right)$. 


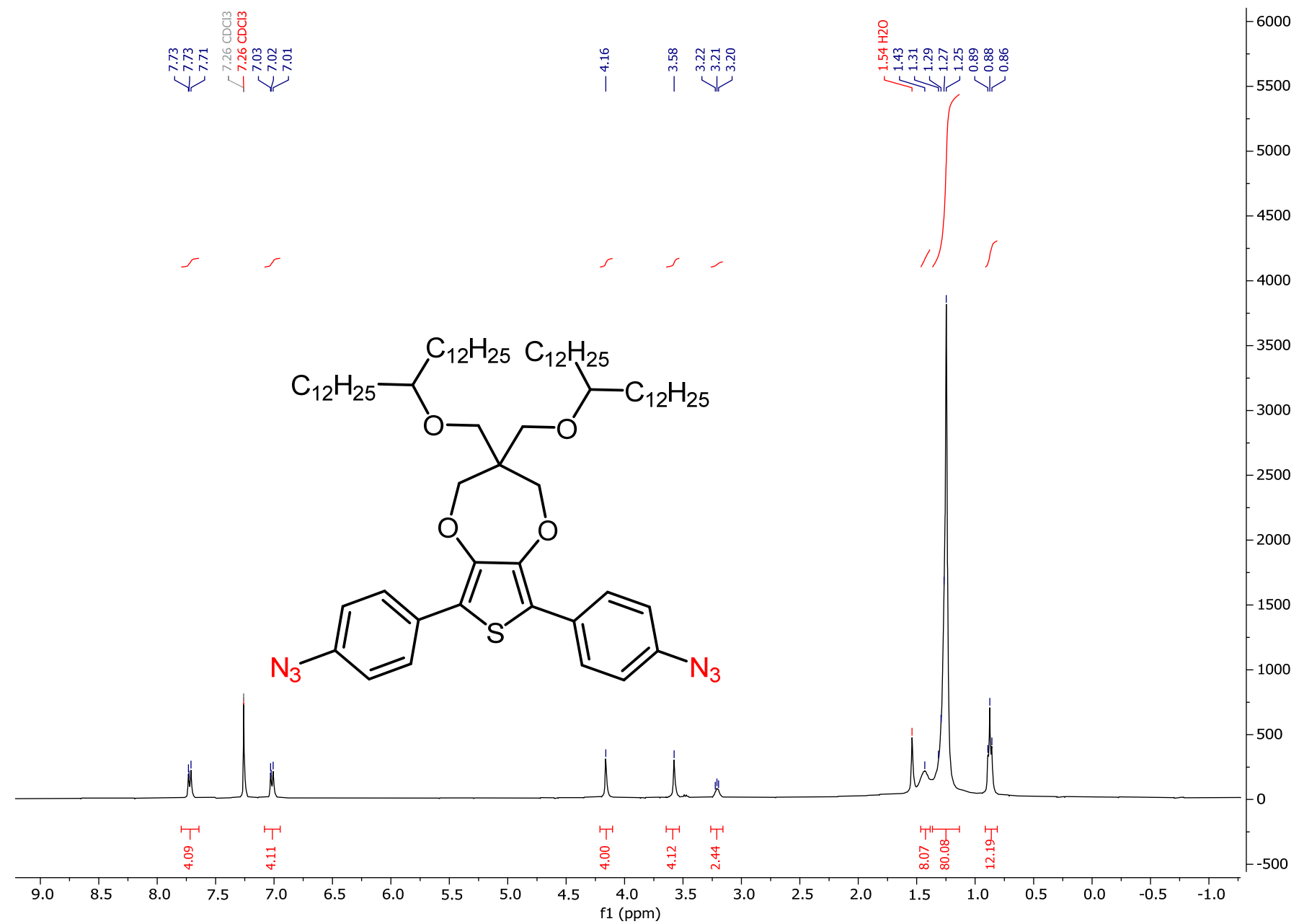

Figure S57. ${ }^{1} \mathrm{H}$ NMR spectrum of $\mathbf{S 1 0}\left(400 \mathrm{MHz}, \mathrm{CDCl}_{3}\right)$. 


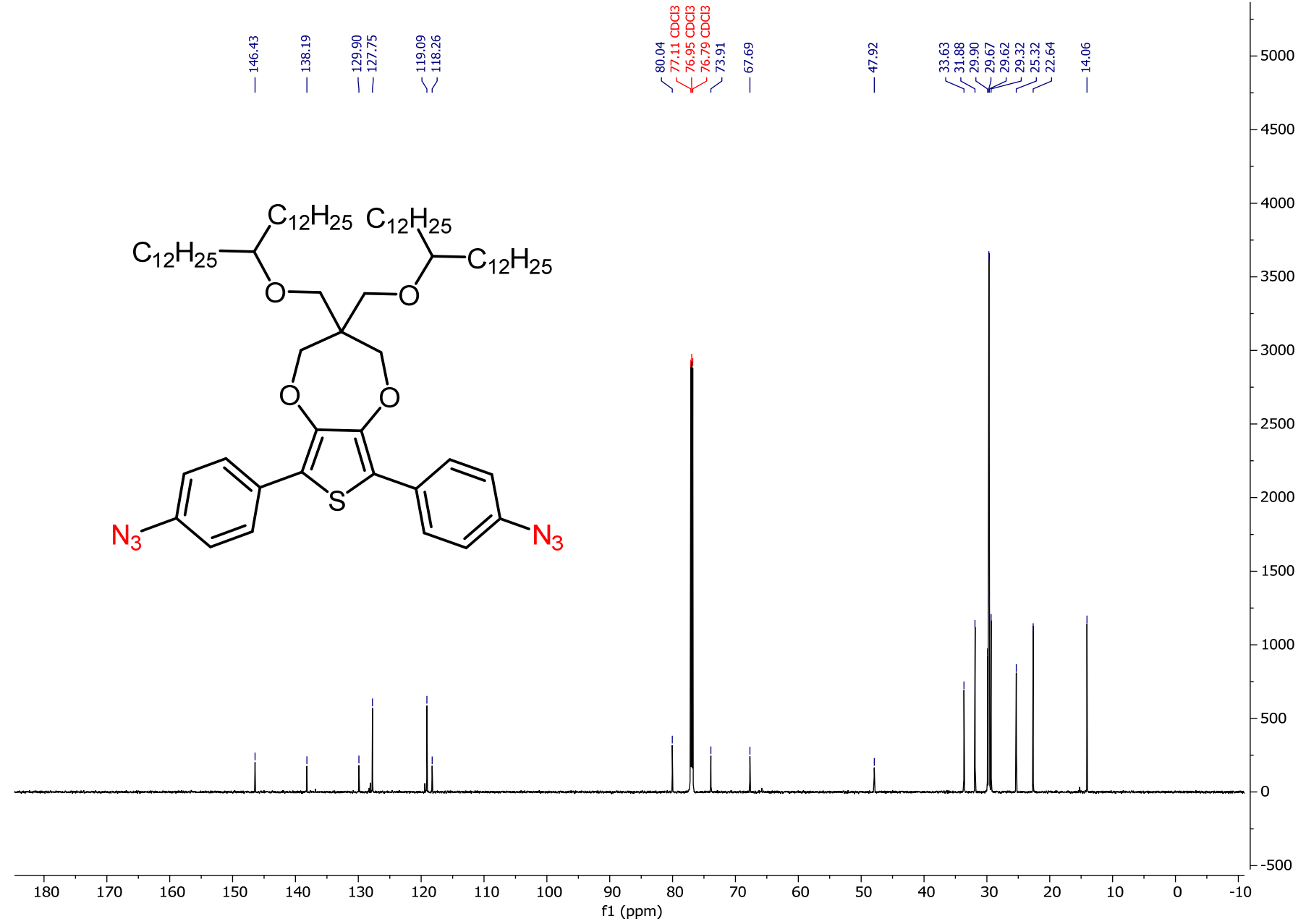

Figure S58. ${ }^{13} \mathrm{C}\left\{{ }^{1} \mathrm{H}\right\}$ NMR spectrum of $\mathbf{S 1 0}\left(201 \mathrm{MHz}, \mathrm{CDCl}_{3}\right)$. 


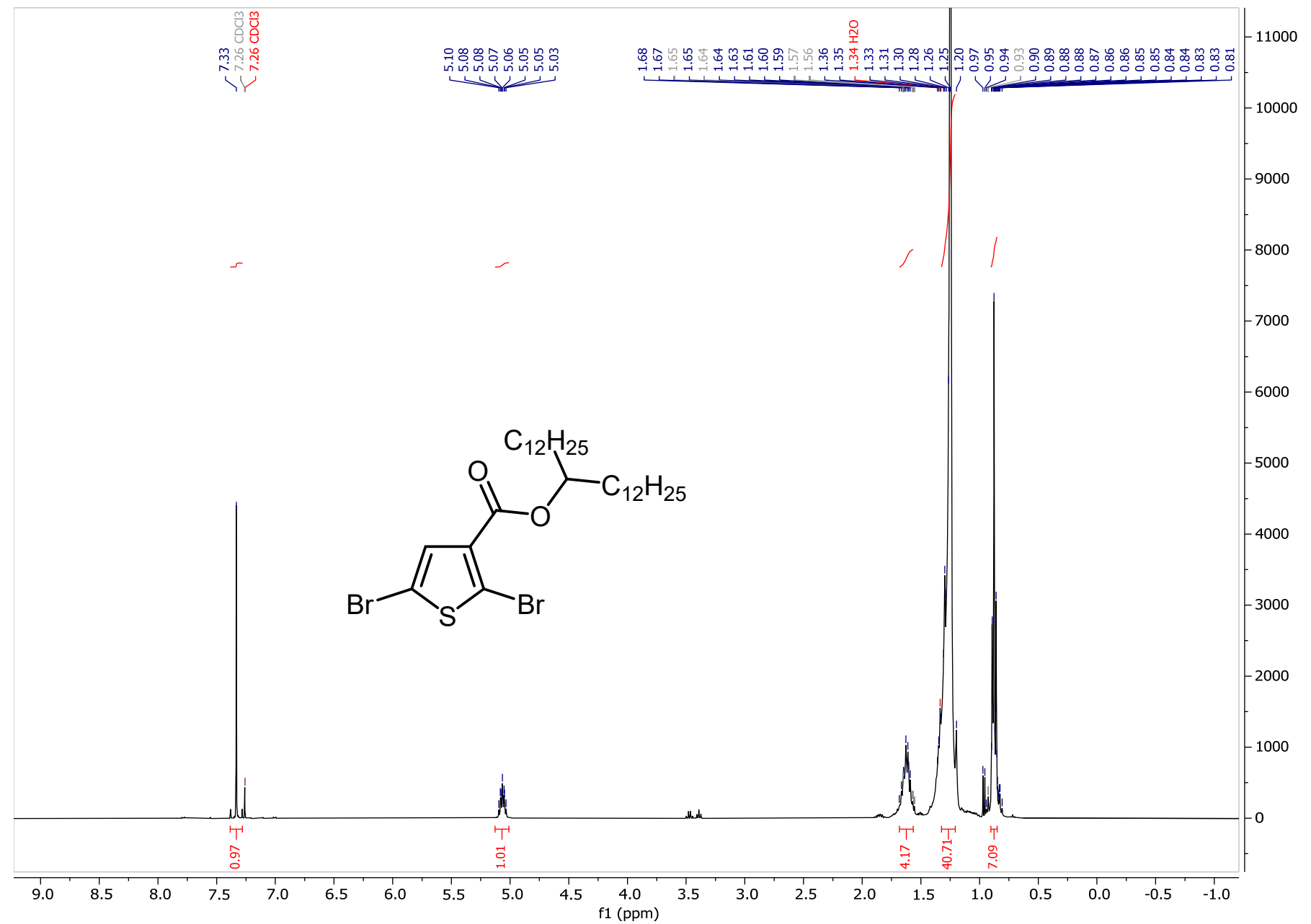

Figure S59. ${ }^{1} \mathrm{H}$ NMR spectrum of $\mathbf{S 1 1}\left(400 \mathrm{MHz}, \mathrm{CDCl}_{3}\right)$. 


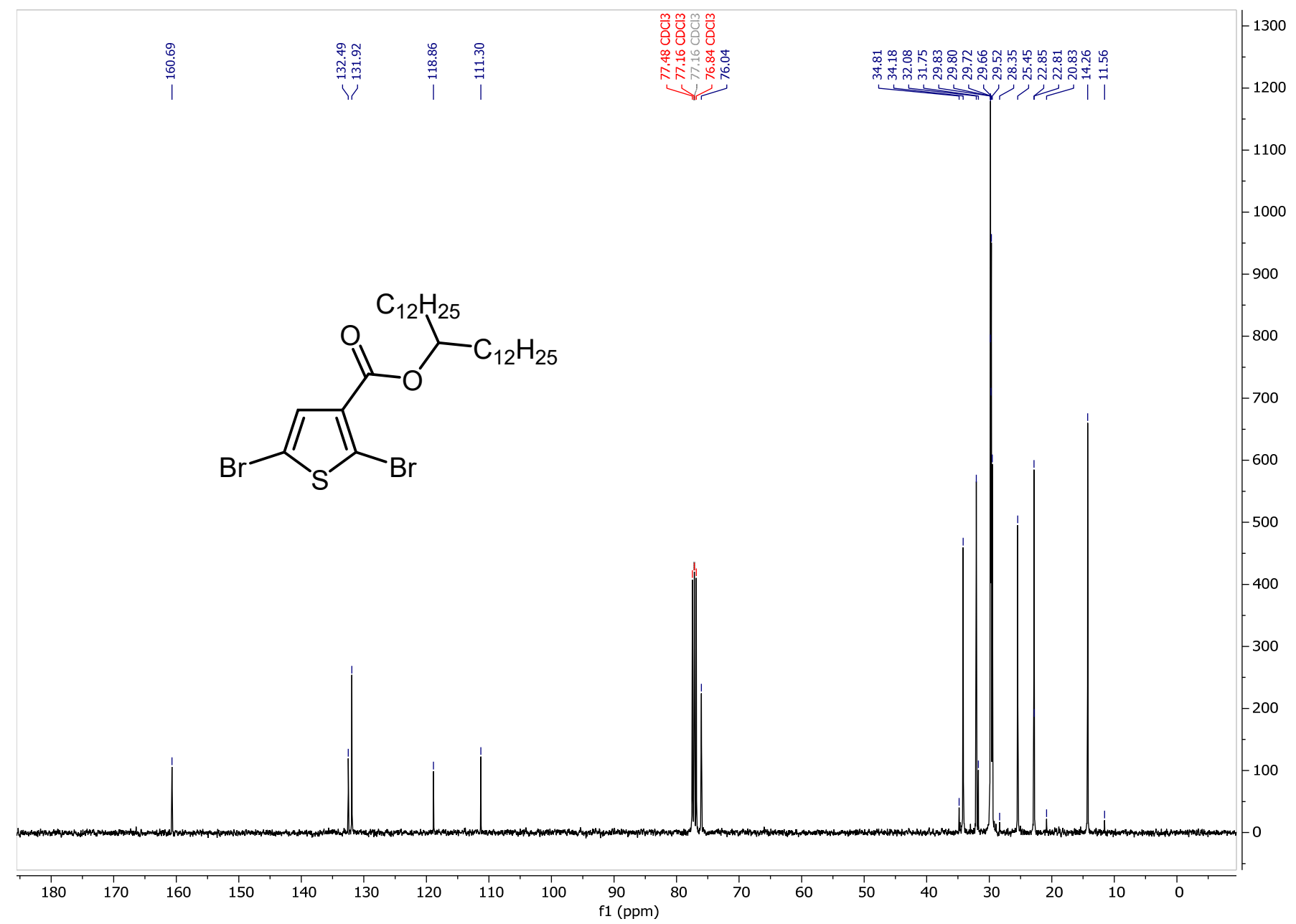

Figure S60. ${ }^{13} \mathrm{C}\left\{{ }^{1} \mathrm{H}\right\}$ NMR spectrum of $\mathbf{S 1 1}\left(101 \mathrm{MHz}, \mathrm{CDCl}_{3}\right)$. 


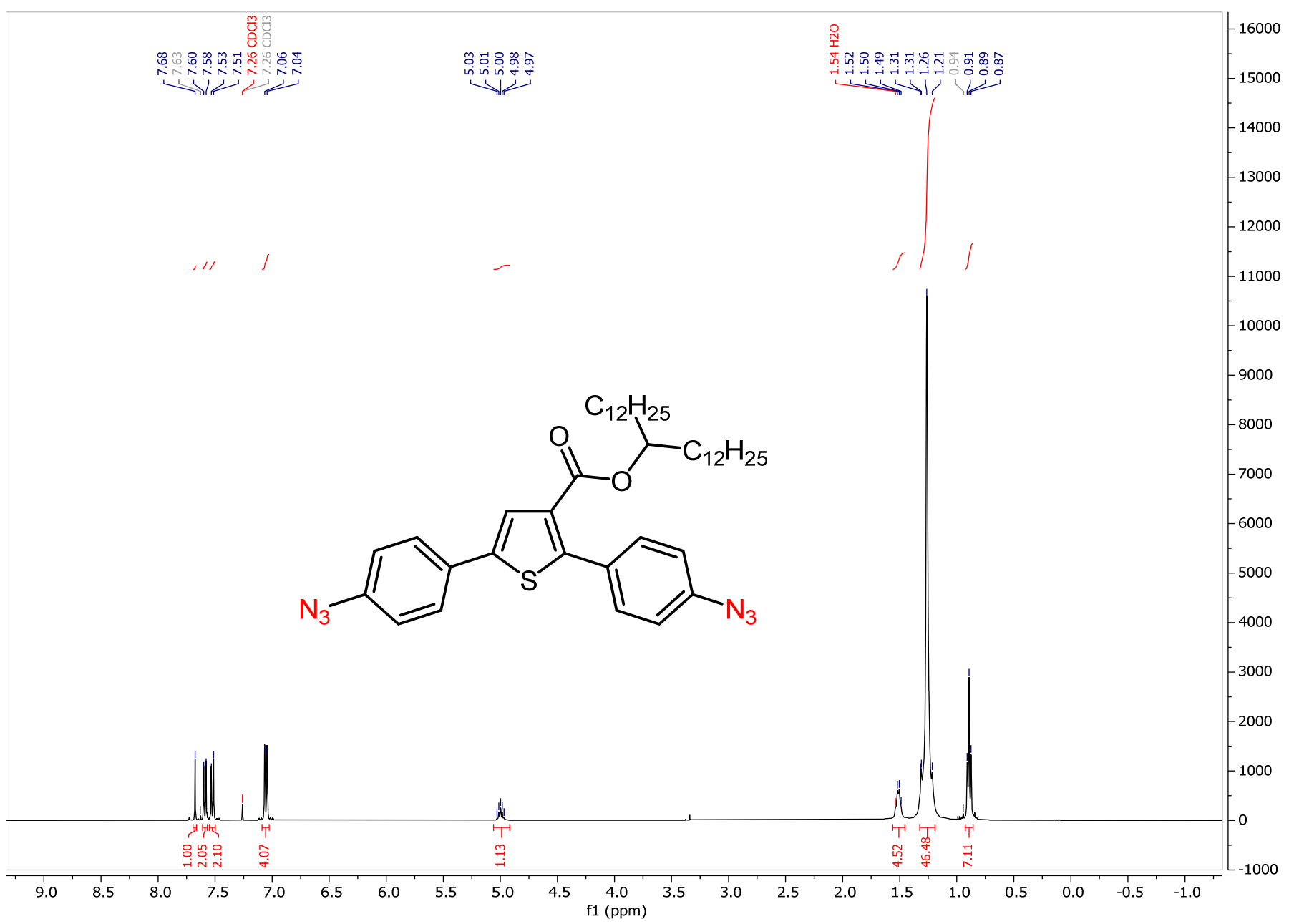

Figure S61. ${ }^{1} \mathrm{H}$ NMR spectrum of $\mathbf{S 1 2}\left(400 \mathrm{MHz}, \mathrm{CDCl}_{3}\right)$. 


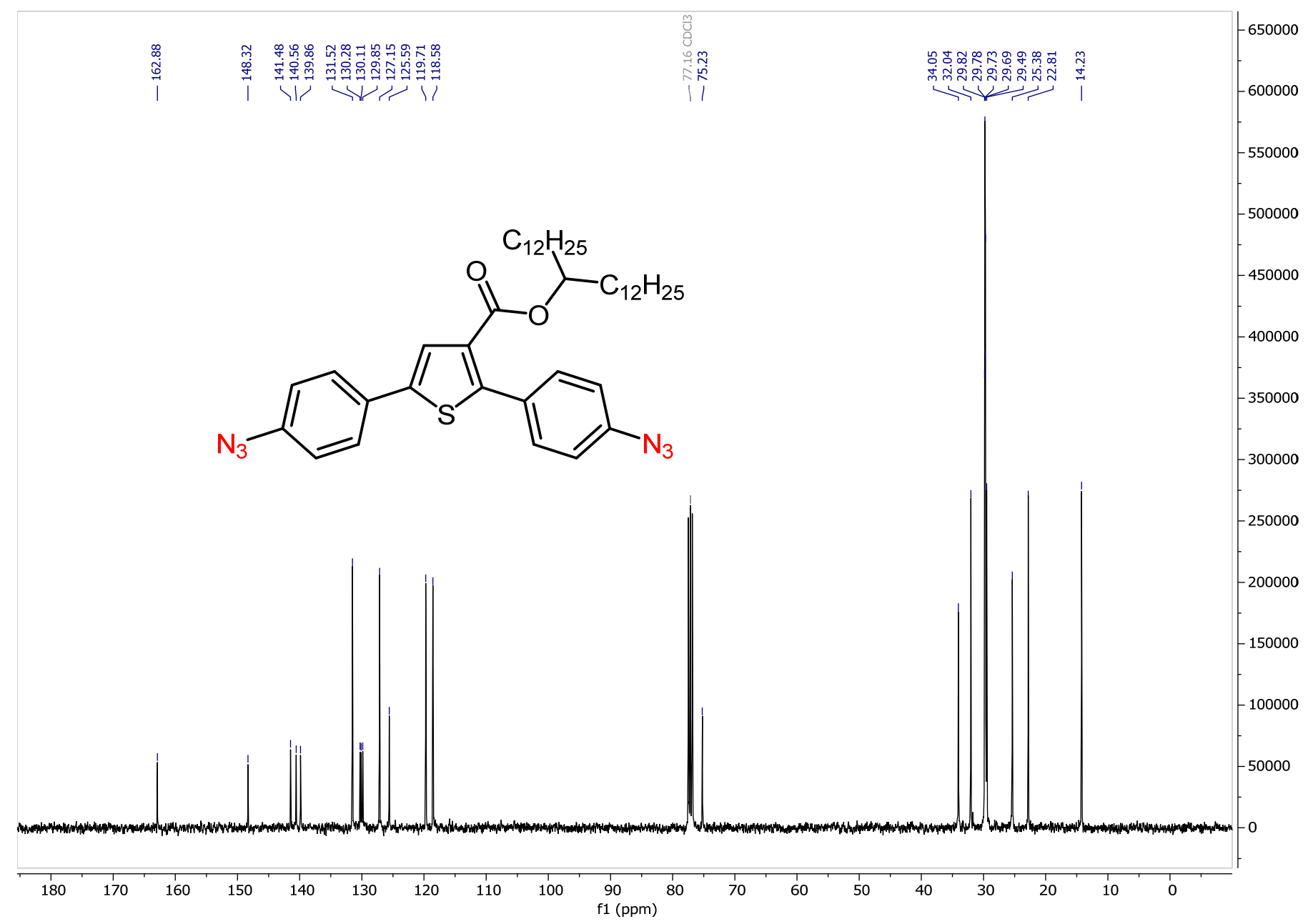

Figure S62. ${ }^{13} \mathrm{C}\left\{{ }^{1} \mathrm{H}\right\}$ NMR spectrum of $\mathbf{S 1 2}\left(101 \mathrm{MHz}, \mathrm{CDCl}_{3}\right)$. 


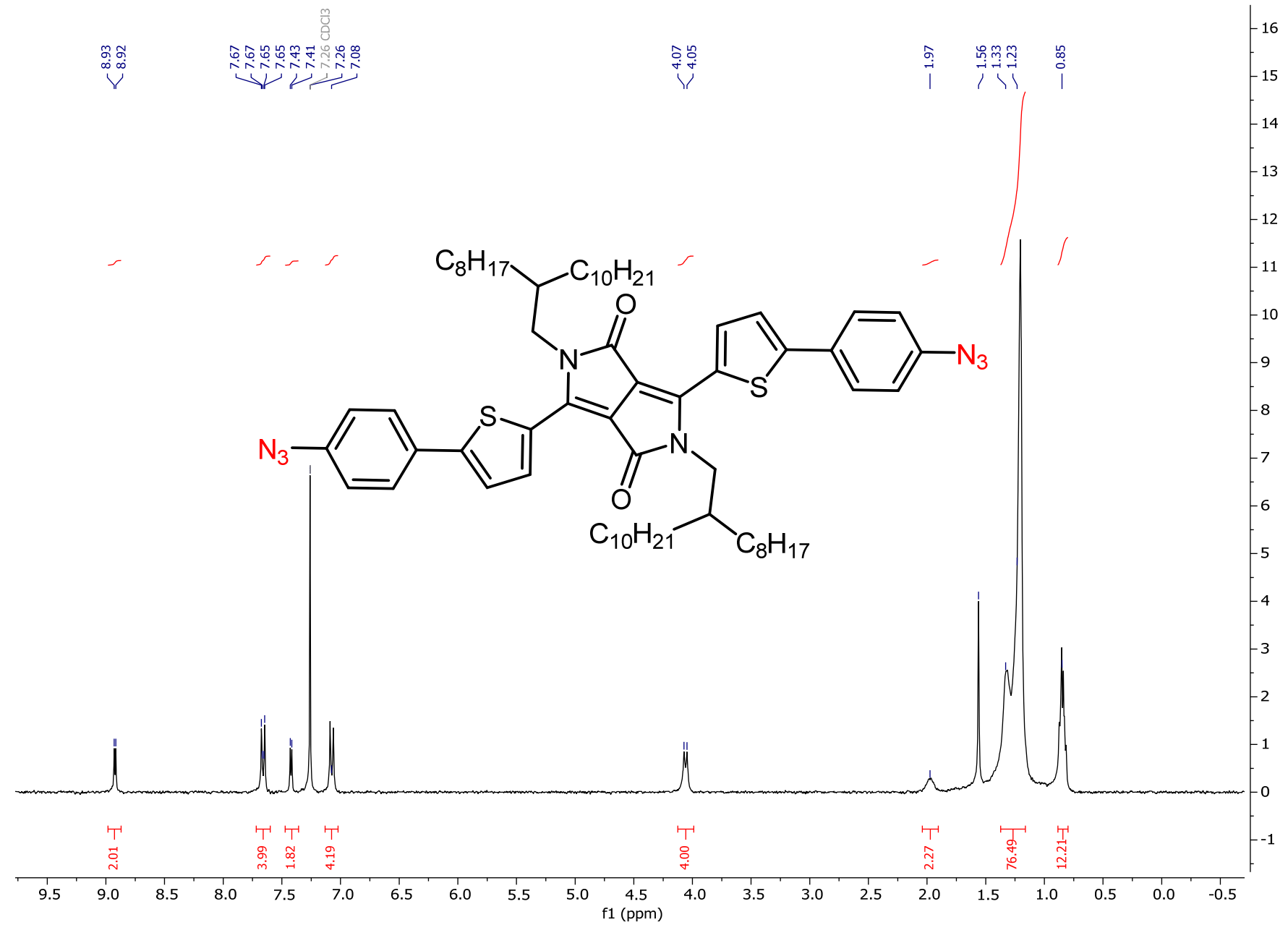

Figure S63. ${ }^{1} \mathrm{H}$ NMR spectrum of $\mathbf{S 1 3}\left(300 \mathrm{MHz}, \mathrm{CDCl}_{3}\right)$. 


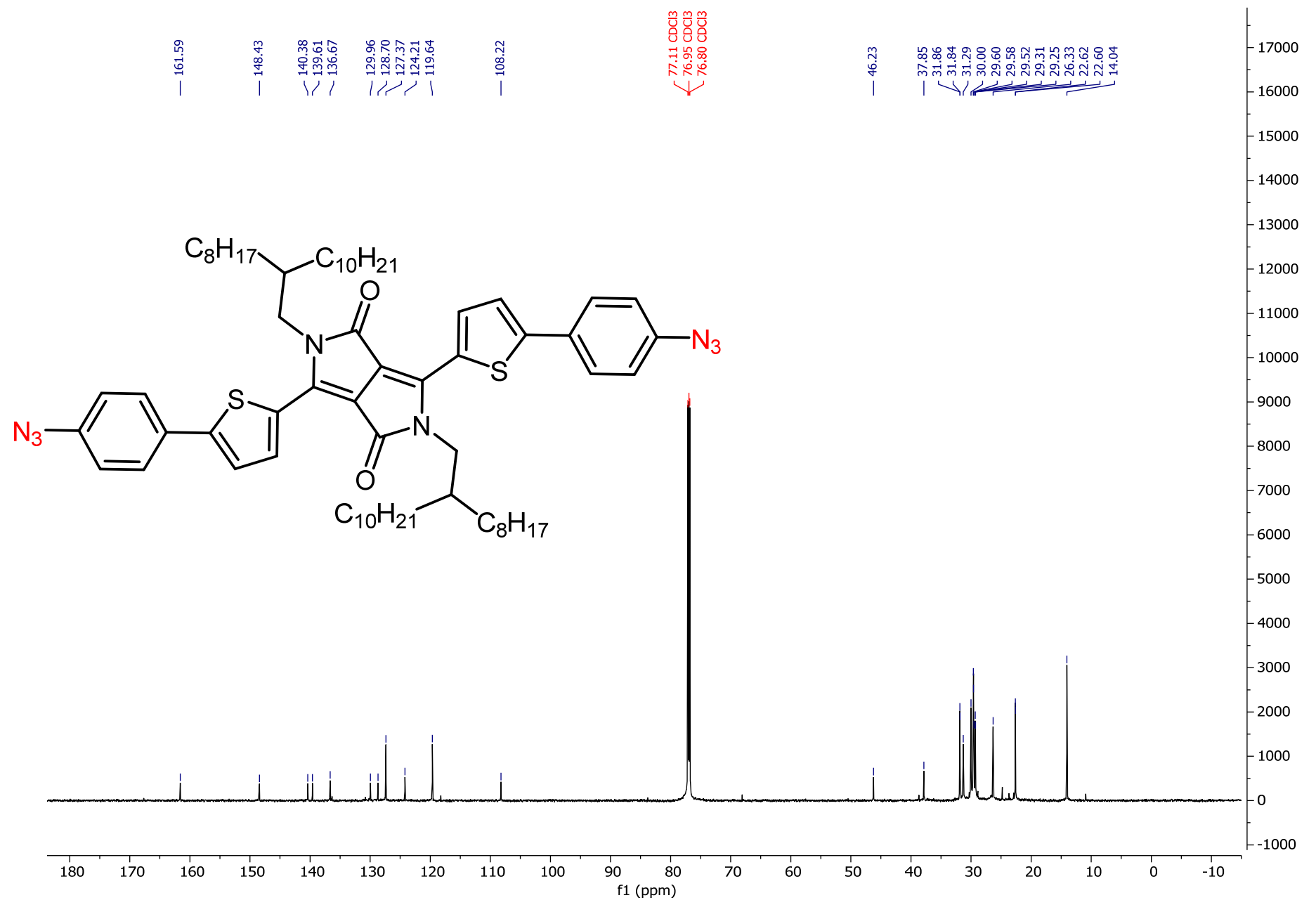

Figure S64. ${ }^{13} \mathrm{C}\left\{{ }^{1} \mathrm{H}\right\}$ NMR spectrum of $\mathbf{S 1 3}\left(201 \mathrm{MHz}, \mathrm{CDCl}_{3}\right)$. 


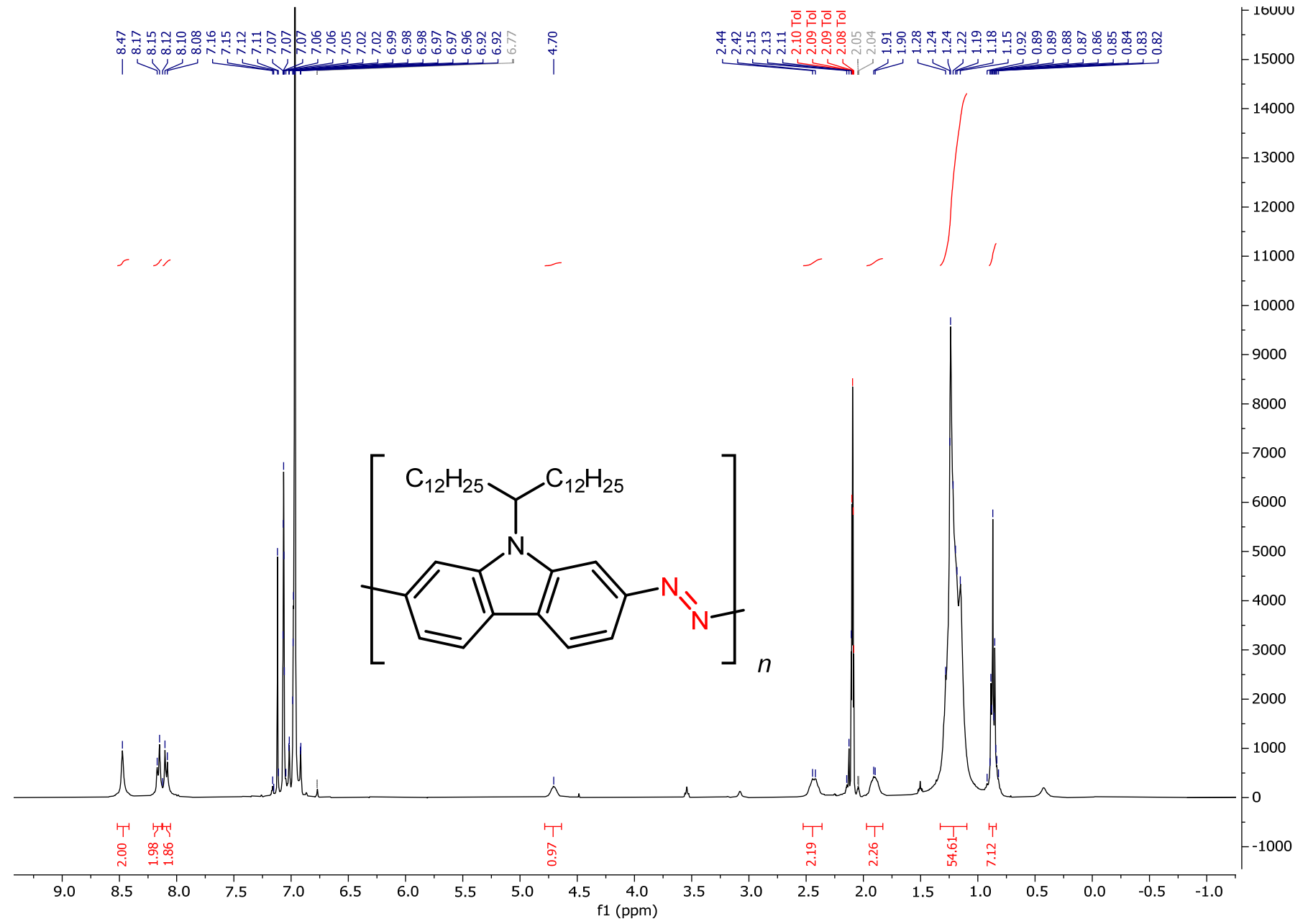

Figure S65. ${ }^{1} \mathrm{H}$ NMR spectrum of $3\left(400 \mathrm{MHz}, 100{ }^{\circ} \mathrm{C}\right.$, toluene- $\left.d 8\right)$. 


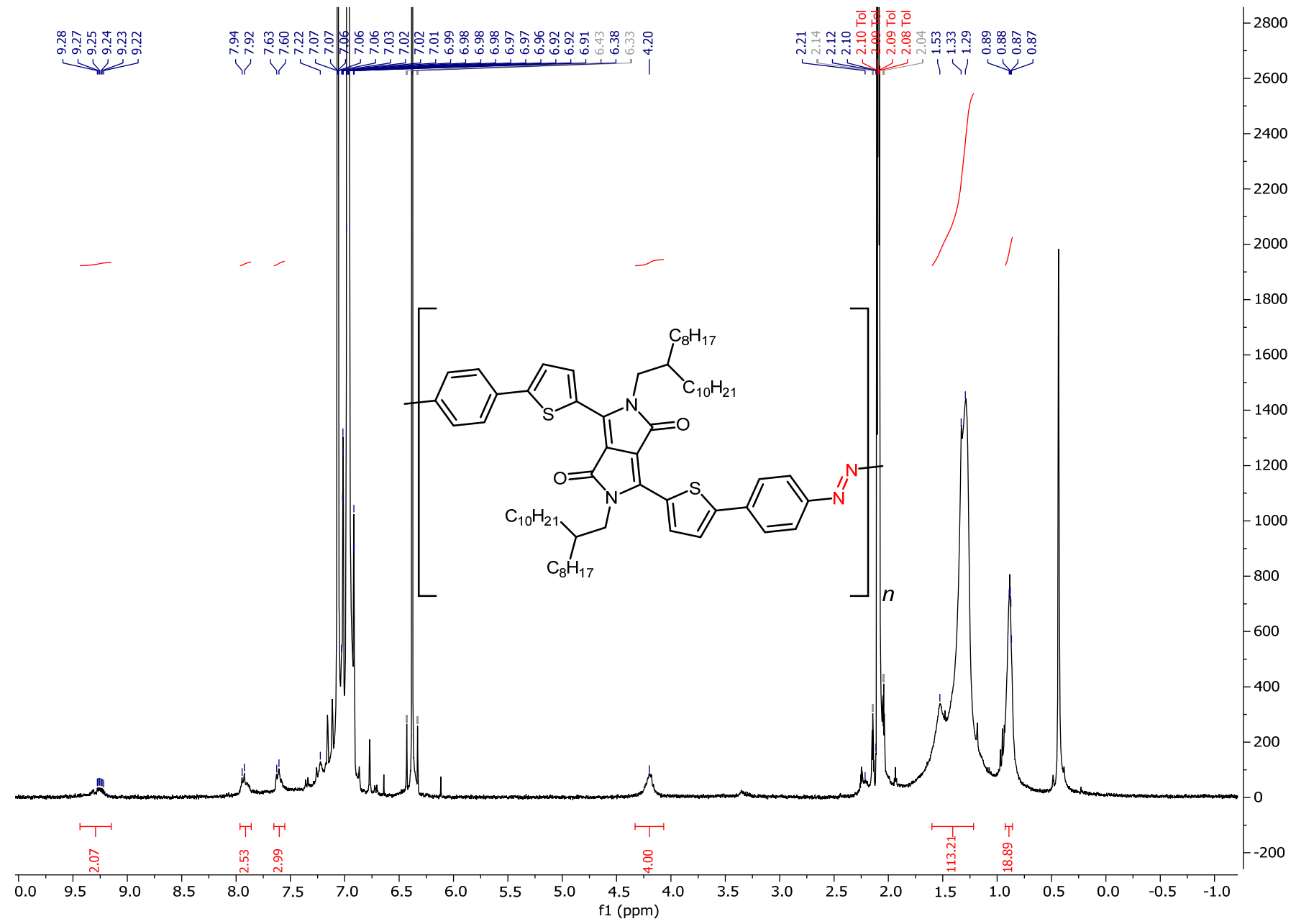

Figure S66. ${ }^{1} \mathrm{H}$ NMR spectrum of 7 ( $400 \mathrm{MHz}, 100{ }^{\circ} \mathrm{C}$, toluene- $d_{8}$ ). 


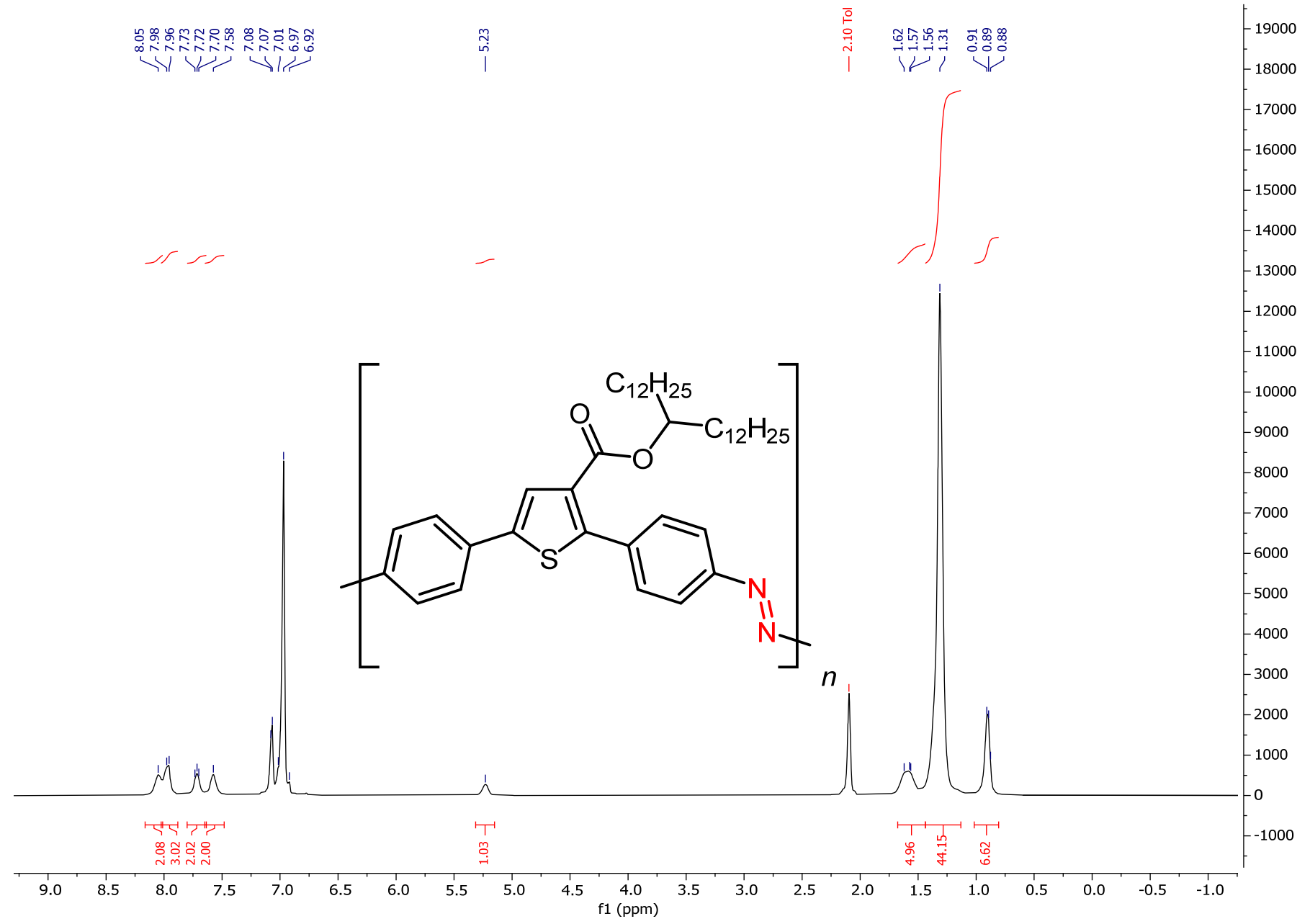

Figure S67. ${ }^{1} \mathrm{H}$ NMR spectrum of $8\left(400 \mathrm{MHz}, 100{ }^{\circ} \mathrm{C}\right.$, toluene- $\left.d_{8}\right)$. 


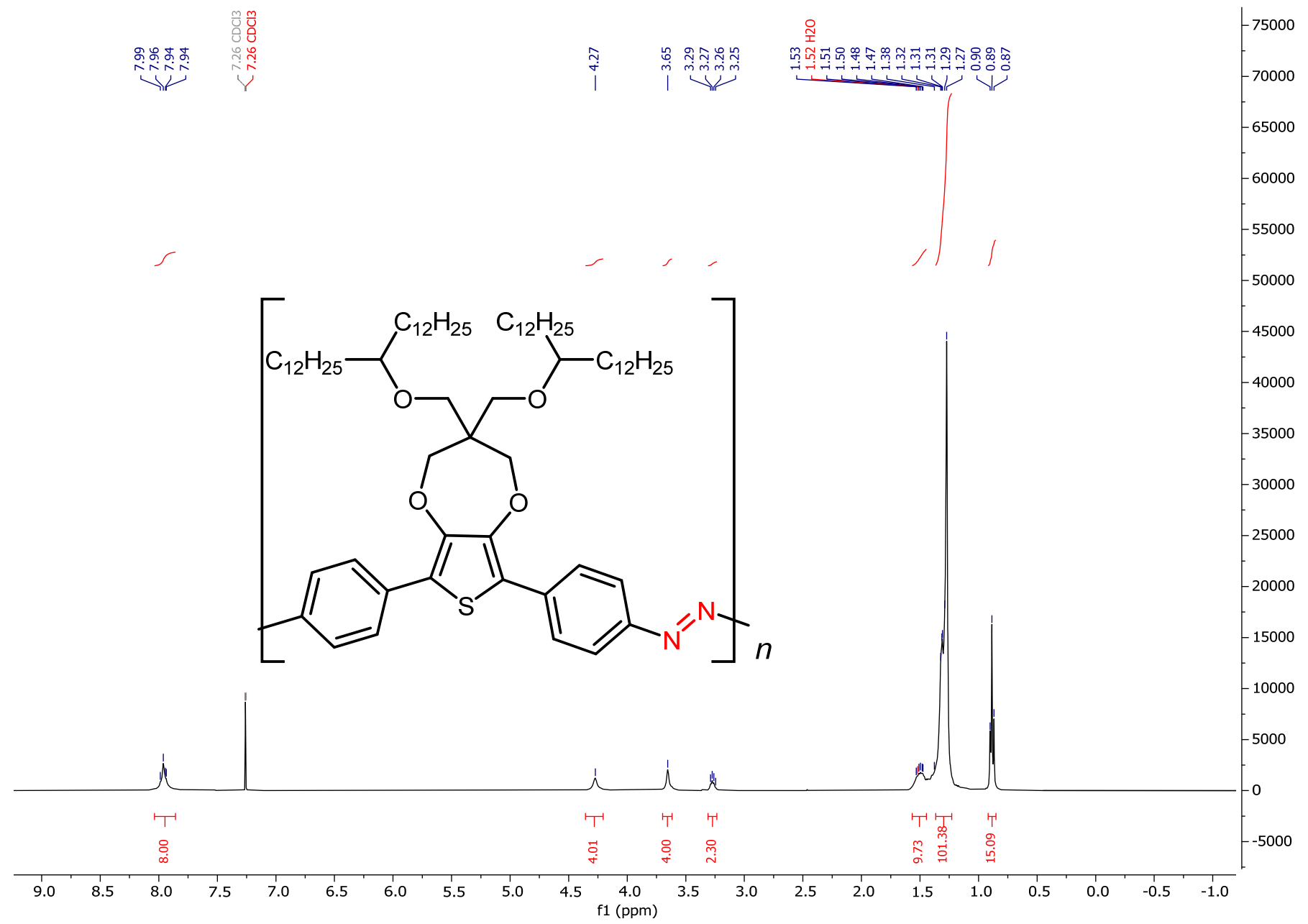

Figure S68. ${ }^{1} \mathrm{H}$ NMR spectrum of $9\left(400 \mathrm{MHz}, 50^{\circ} \mathrm{C}, \mathrm{CDCl}_{3}\right)$. 


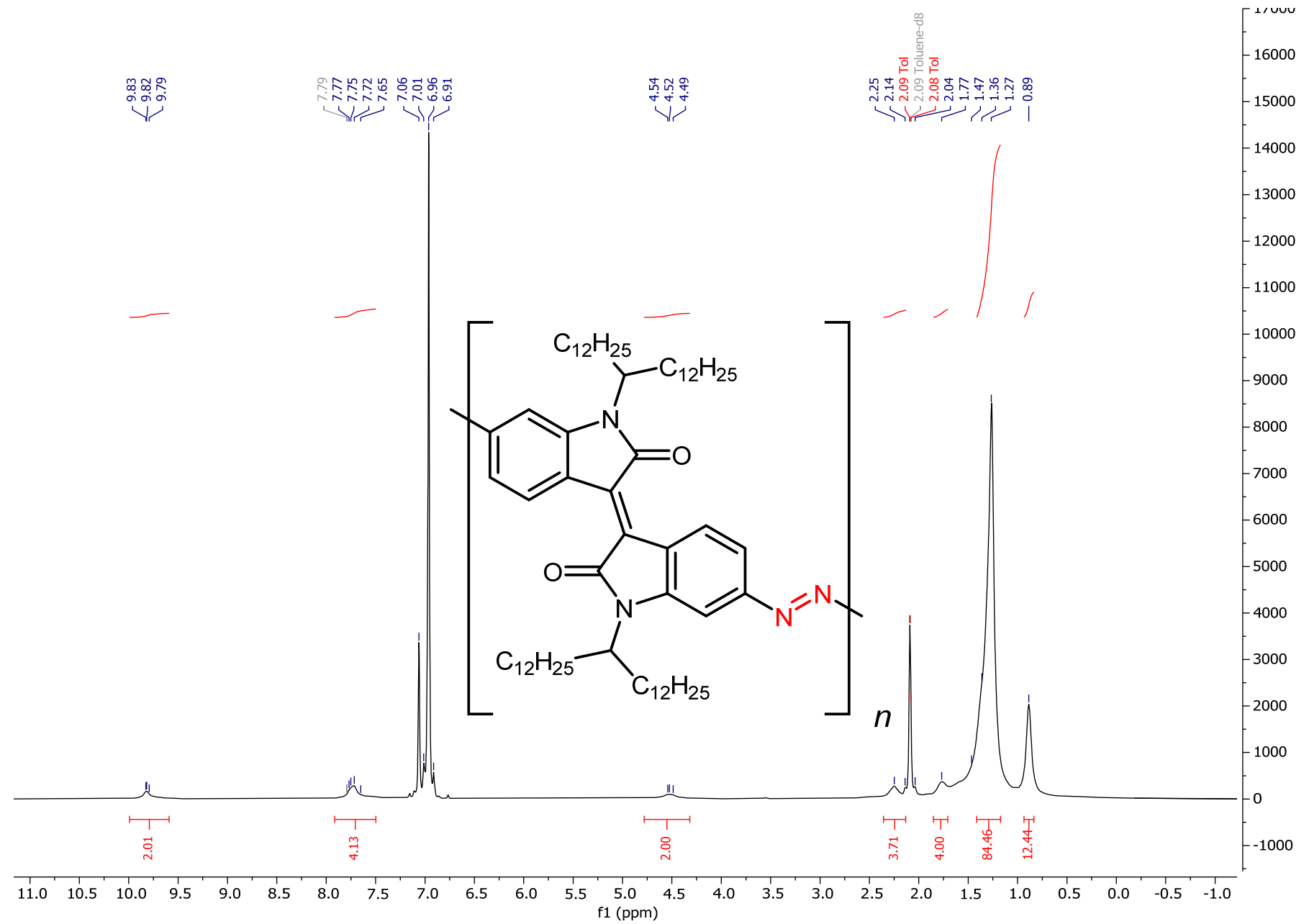

Figure S69. ${ }^{1} \mathrm{H}$ NMR spectrum of $10\left(400 \mathrm{MHz}, 100{ }^{\circ} \mathrm{C}\right.$, toluene- $\left.d 8\right)$. 


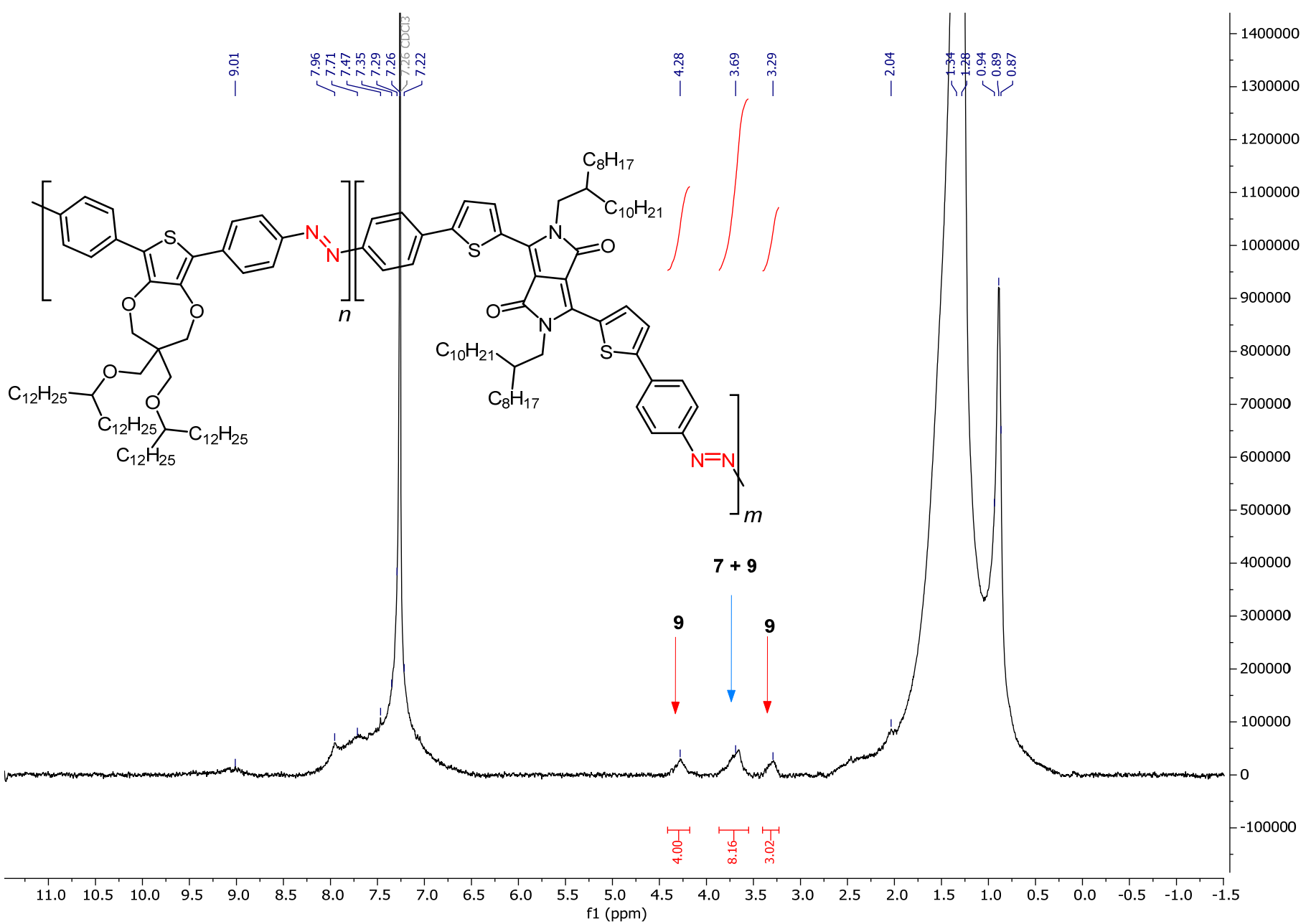

Figure S70. ${ }^{1} \mathrm{H}$ NMR spectrum of $11\left(500 \mathrm{MHz}, 50{ }^{\circ} \mathrm{C}, \mathrm{CDCl}_{3}\right)$. 


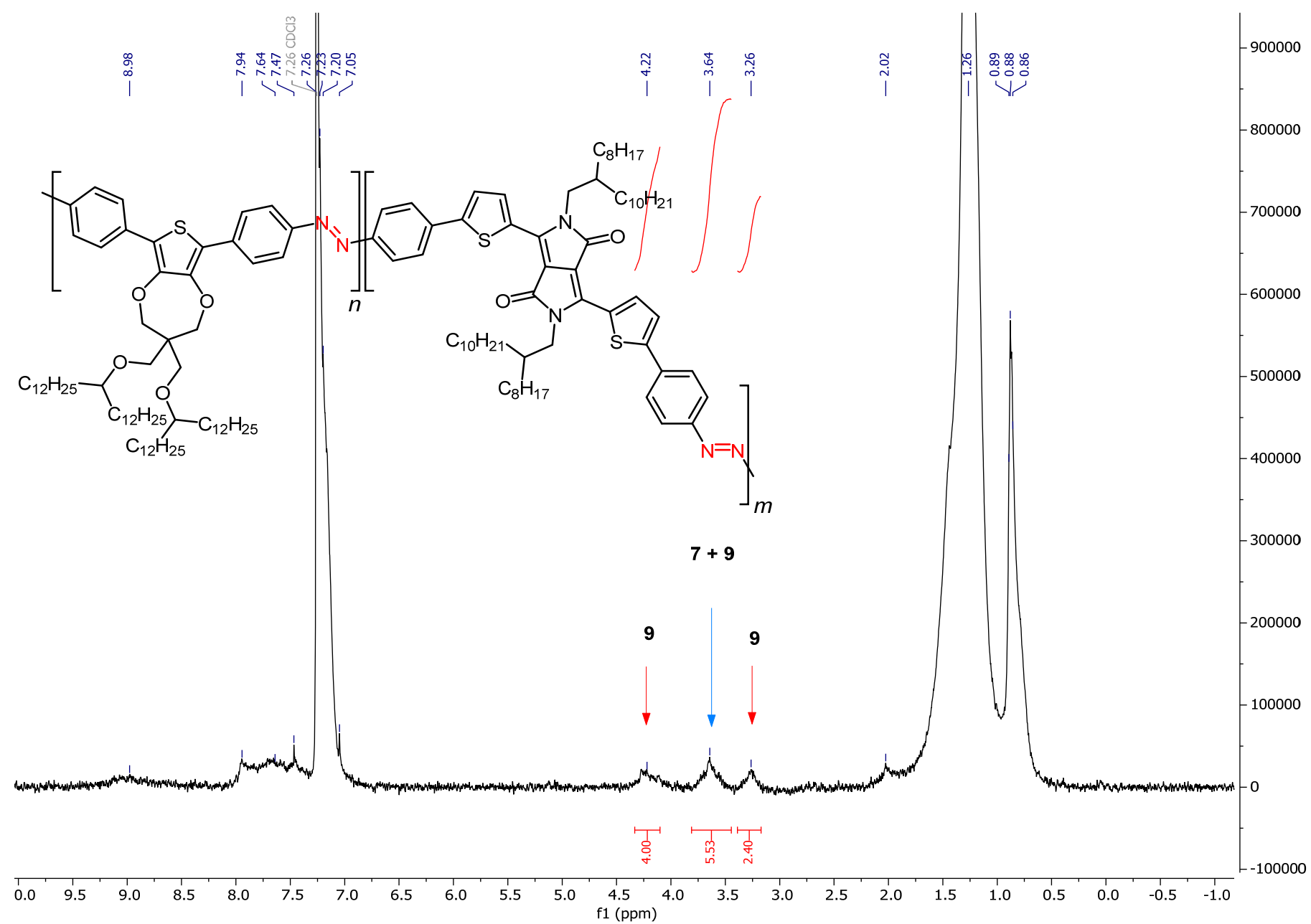

Figure S71. ${ }^{1} \mathrm{H}$ NMR spectrum of $12\left(500 \mathrm{MHz}, 50{ }^{\circ} \mathrm{C}, \mathrm{CDCl}_{3}\right)$. 


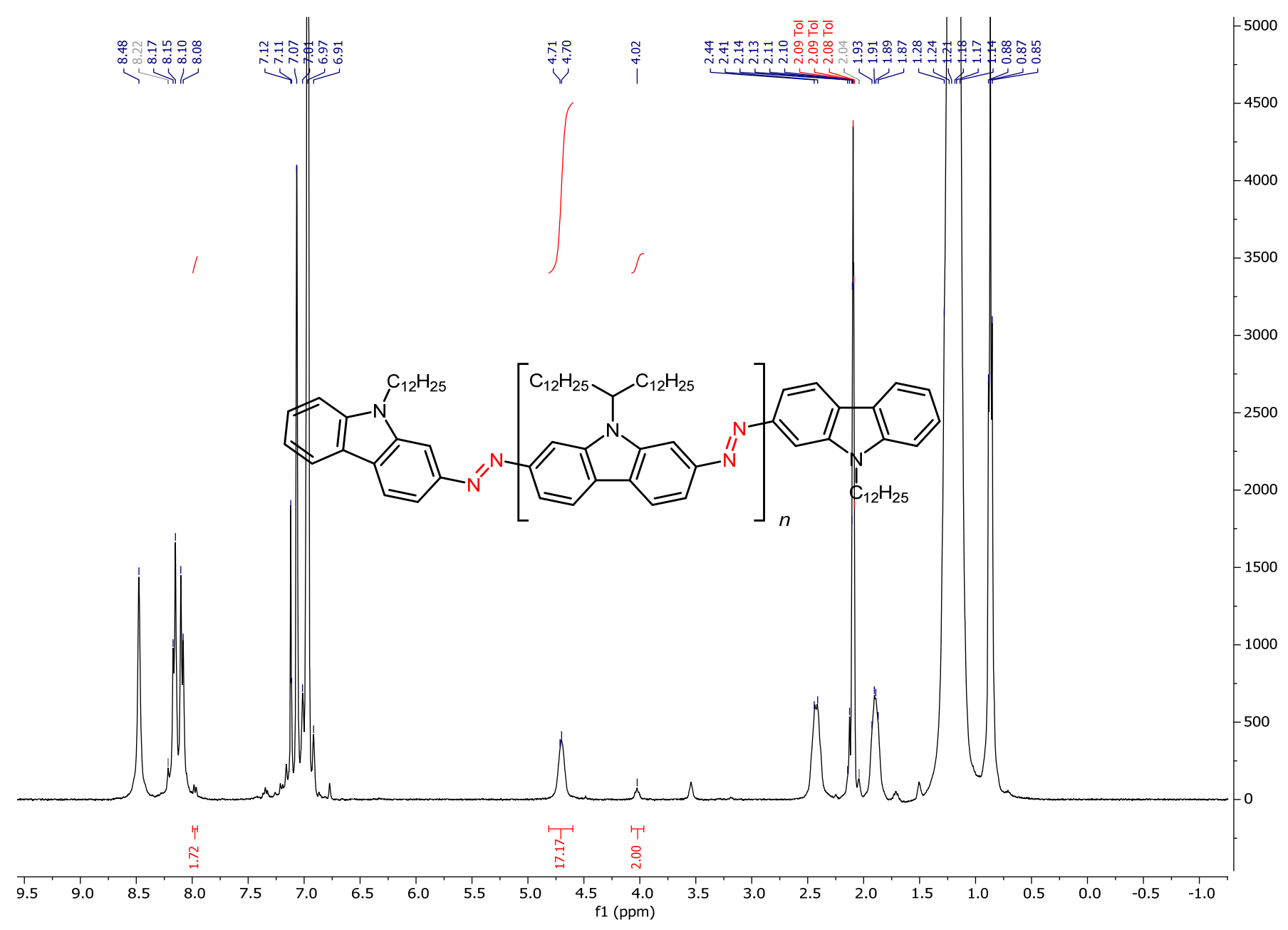

Figure S72. ${ }^{1} \mathrm{H}$ NMR spectrum of $13\left(400 \mathrm{MHz}, 100^{\circ} \mathrm{C}\right.$, toluene- $\left.d_{8}\right)$. 


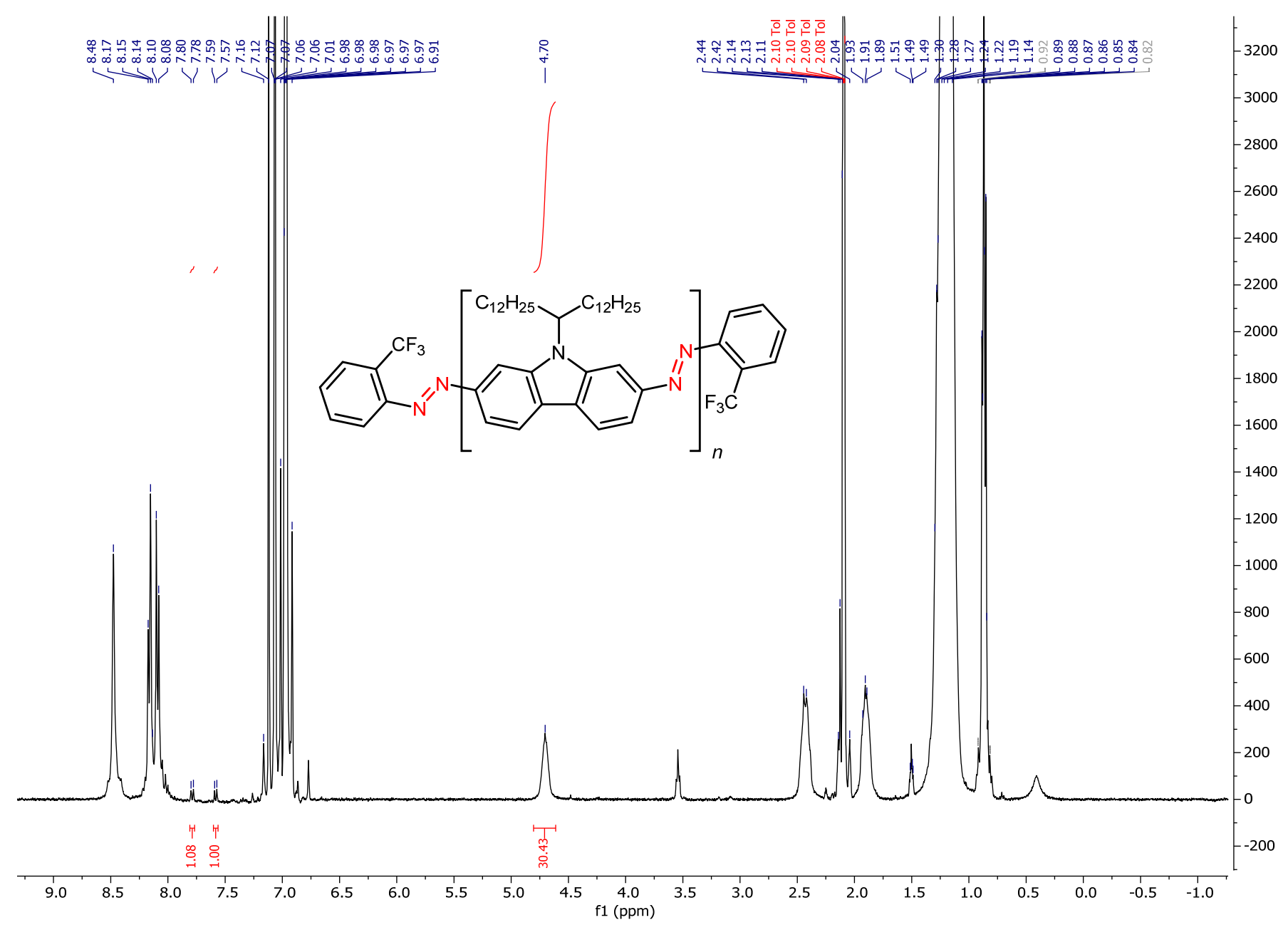

Figure S73. ${ }^{1 \mathrm{H}} \mathrm{NMR}$ spectrum of $14\left(400 \mathrm{MHz}, 100^{\circ} \mathrm{C}\right.$, toluene- $\left.\mathrm{d}_{8}\right)$. 


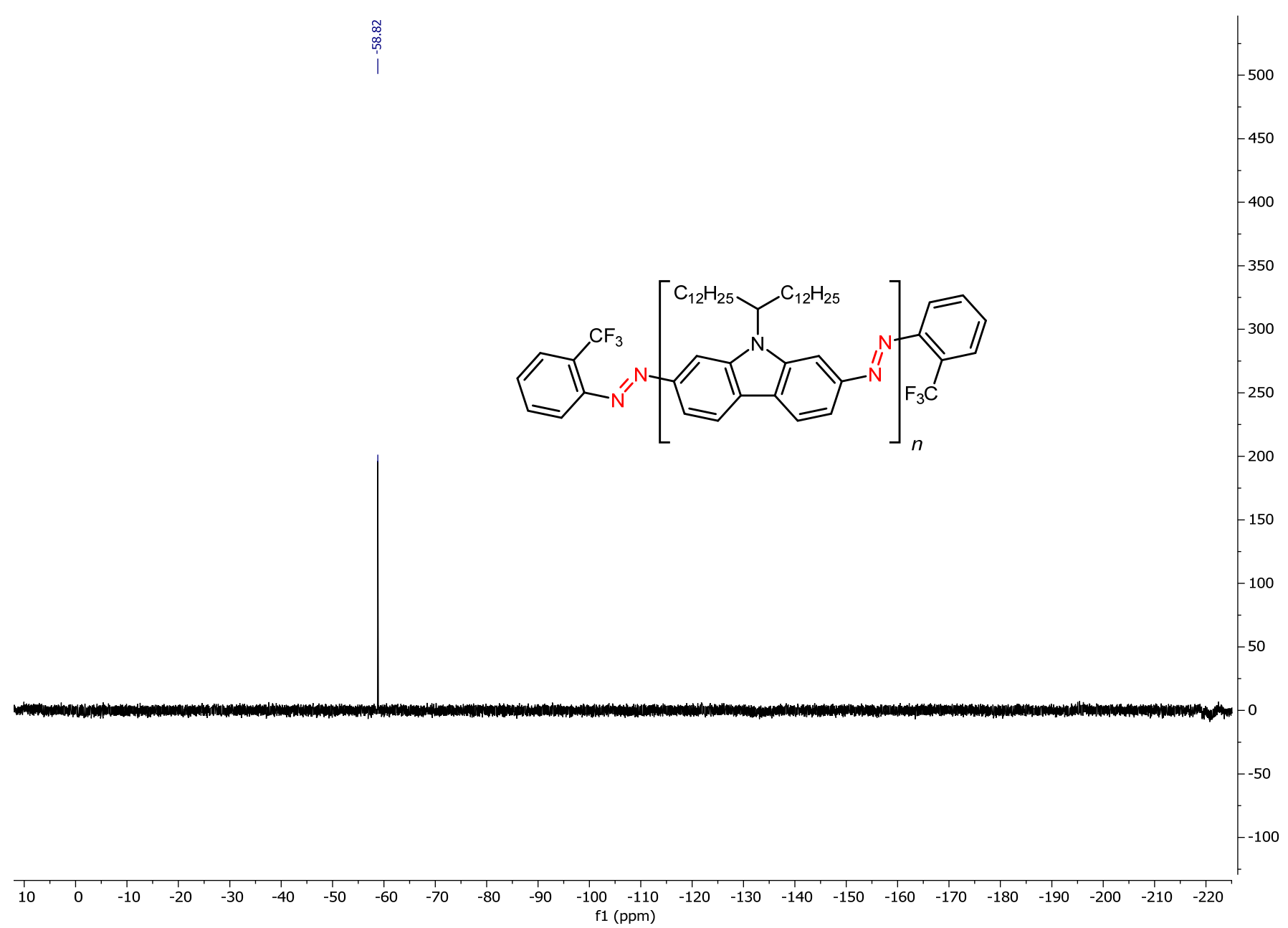

Figure S74. ${ }^{19} \mathrm{~F}$ NMR spectrum of $14\left(376 \mathrm{MHz}, 100{ }^{\circ} \mathrm{C}\right.$, toluene- $\left.\mathrm{d}_{8}\right)$. 


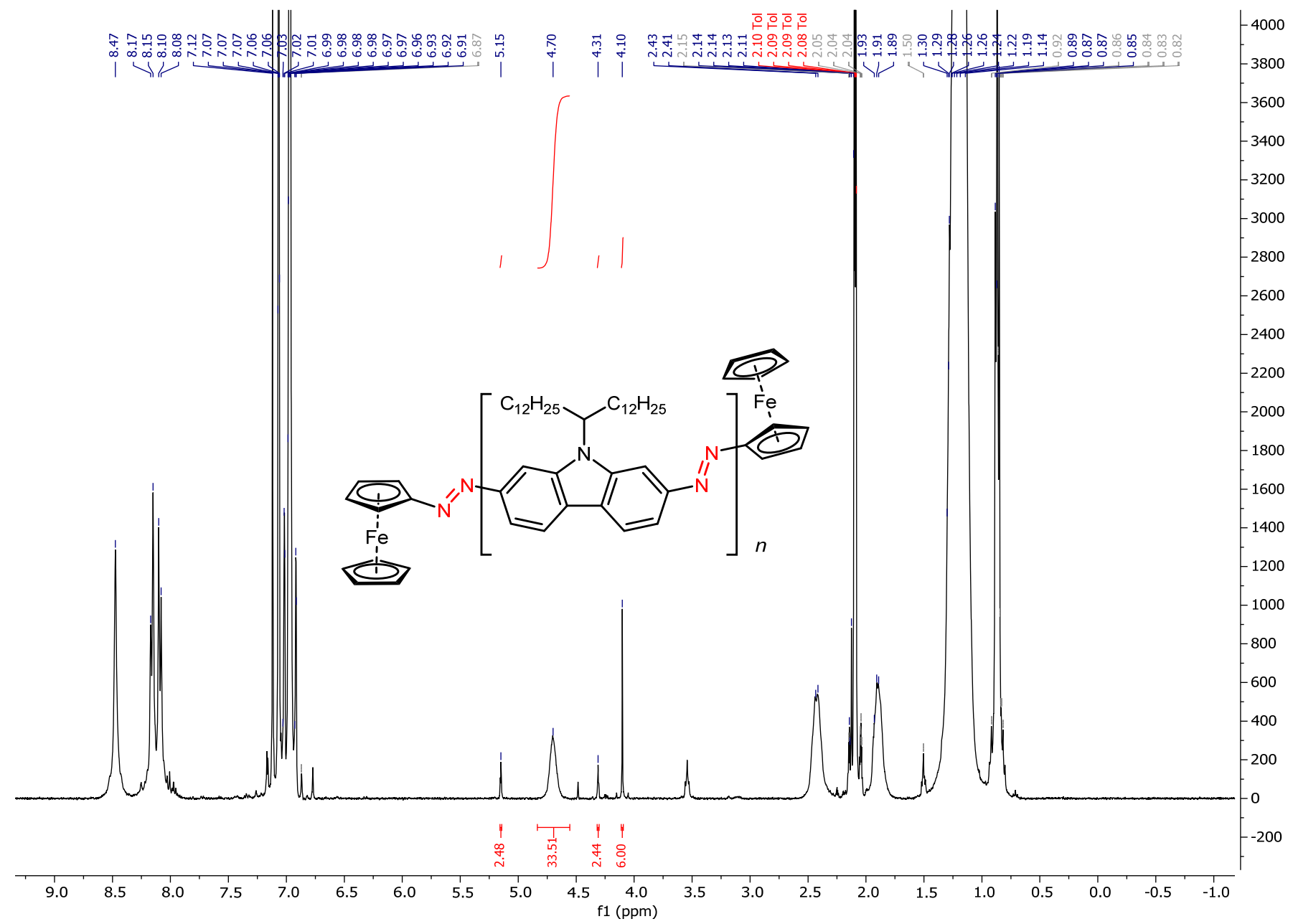

Figure S75. ${ }^{1} \mathrm{H}$ NMR spectrum of $15\left(400 \mathrm{MHz}, 100{ }^{\circ} \mathrm{C}\right.$, toluene- $\left.d 8\right)$. 


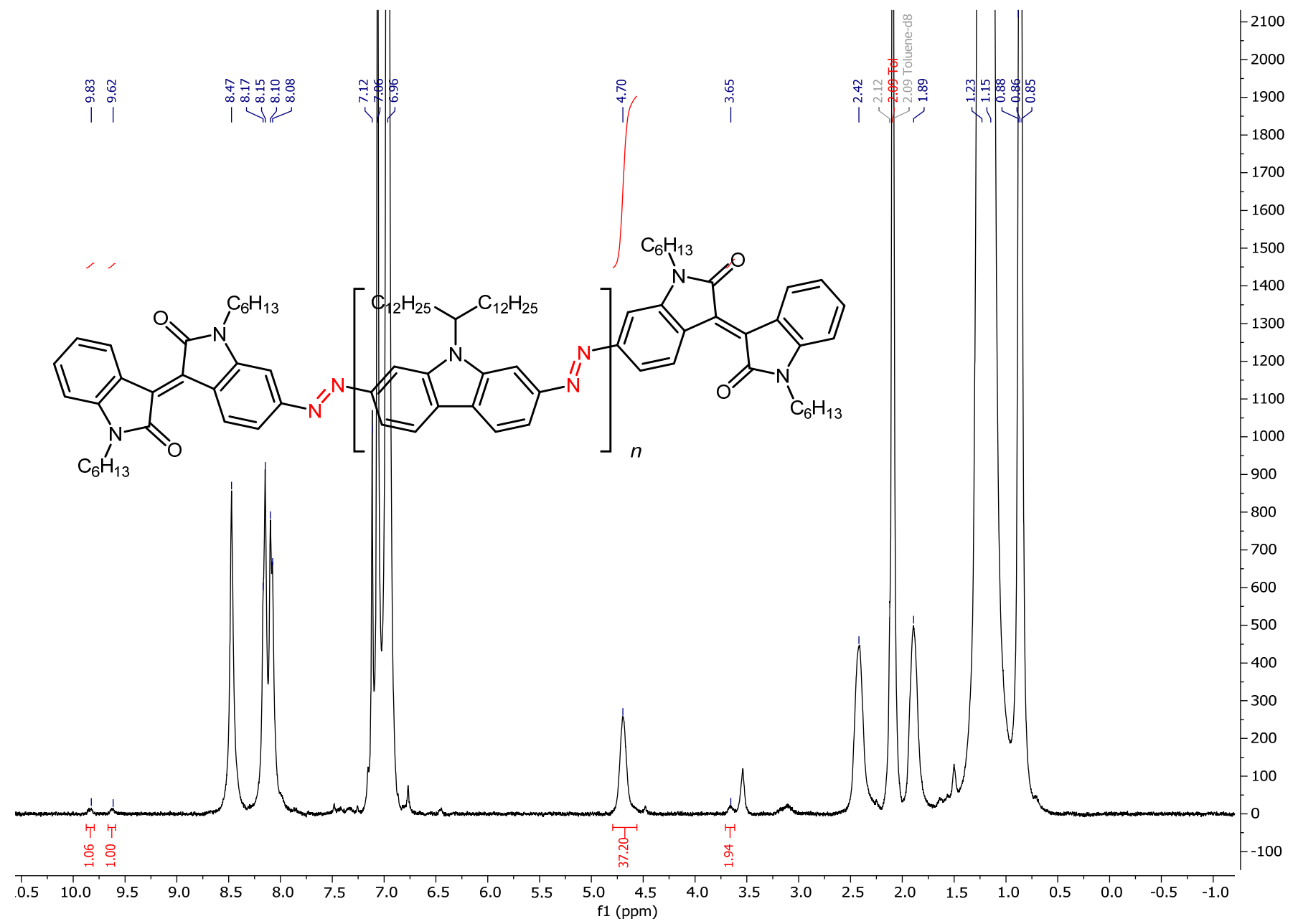

Figure S76. ${ }^{1} \mathrm{H}$ NMR spectrum of $16\left(400 \mathrm{MHz}, 100^{\circ} \mathrm{C}\right.$, toluene- $\left.d_{8}\right)$. 


\section{UV-Vis Spectroscopy}

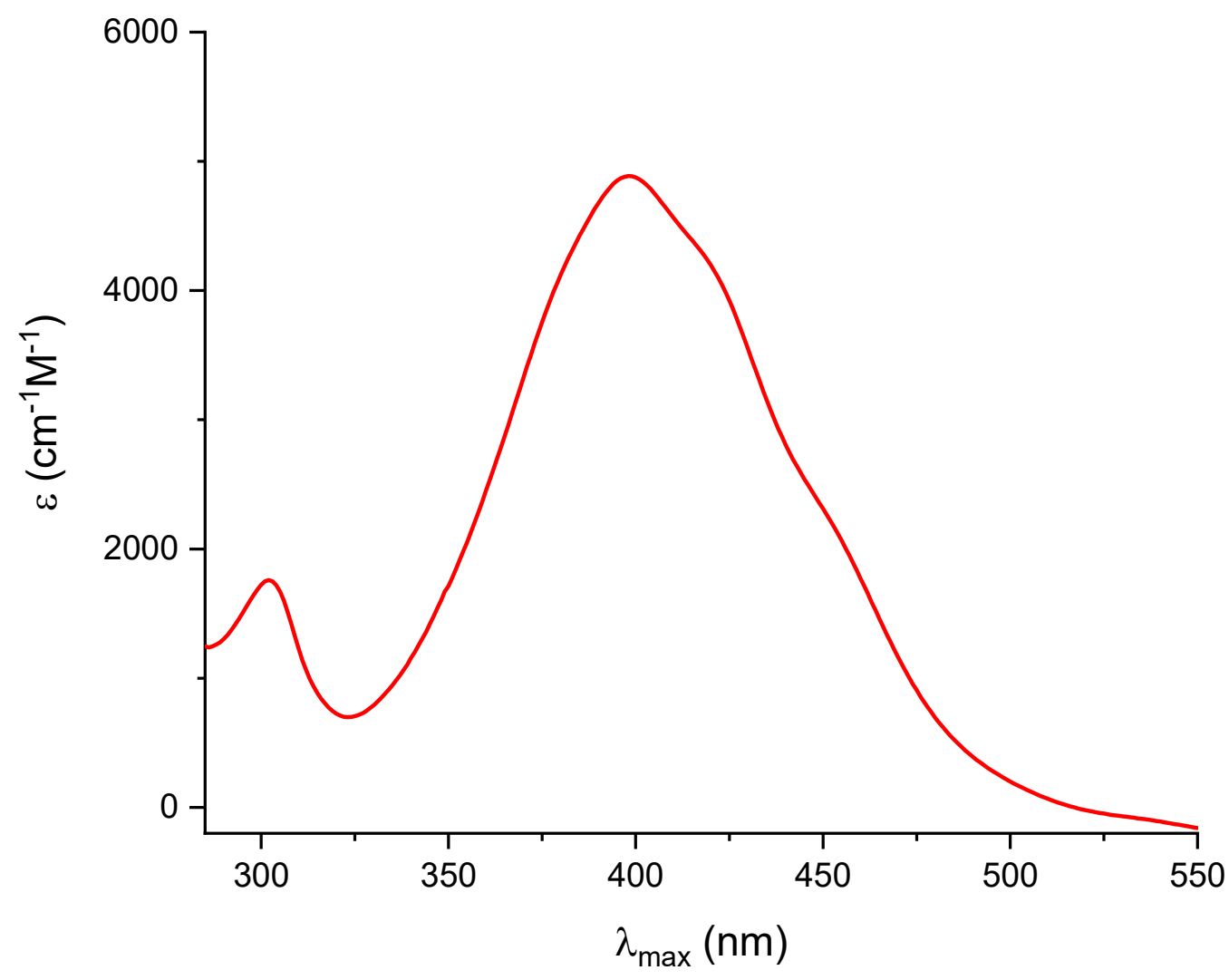

Figure S77. UV-Vis spectrum of $\mathbf{5 b}(0.143 \mathrm{mM})$ in $\mathrm{C}_{6} \mathrm{H}_{5} \mathrm{Cl}$. 


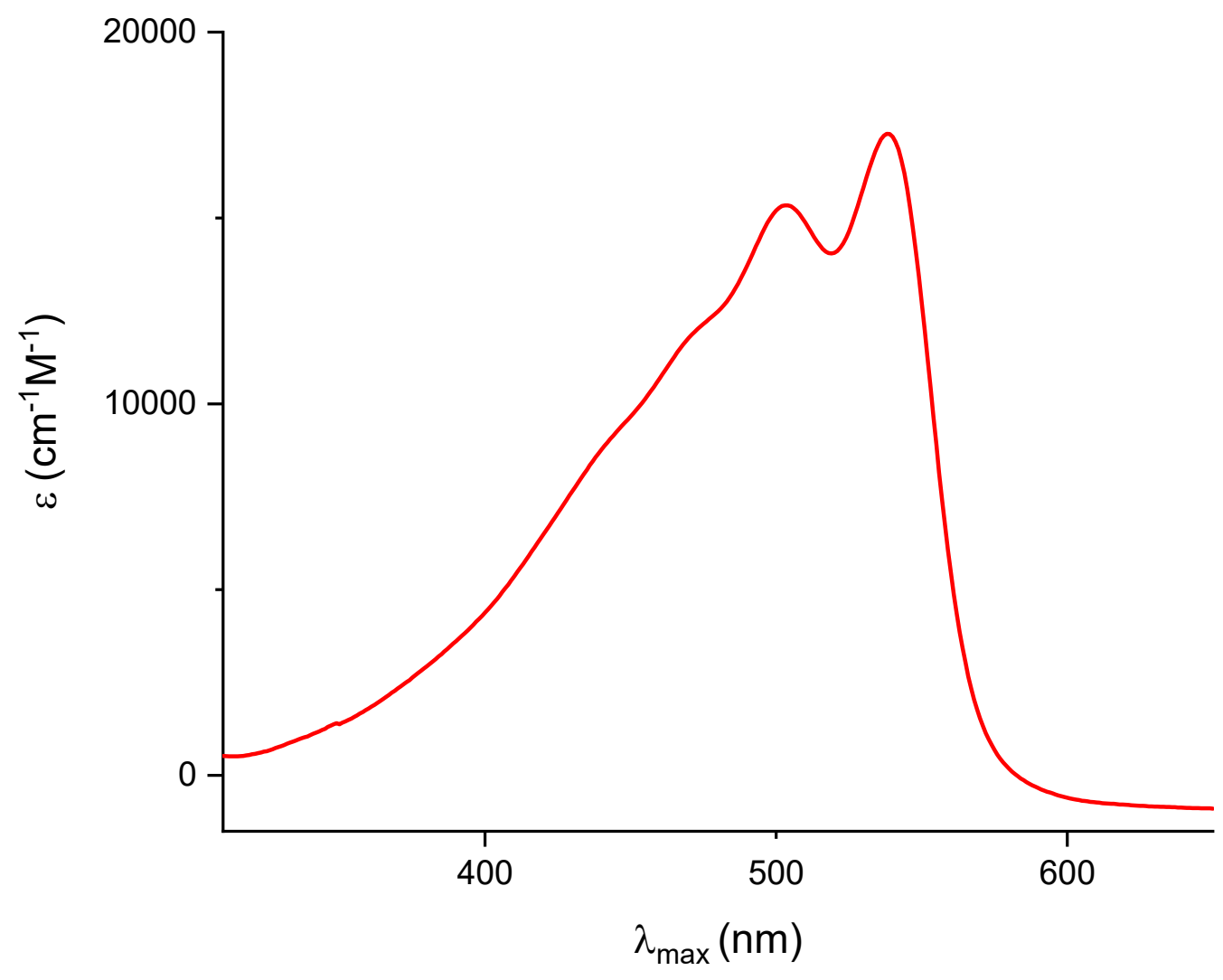

Figure S78. UV-Vis spectrum of $\mathbf{3}(0.0326 \mathrm{mM})$ in $\mathrm{C}_{6} \mathrm{H}_{5} \mathrm{Cl}$. 


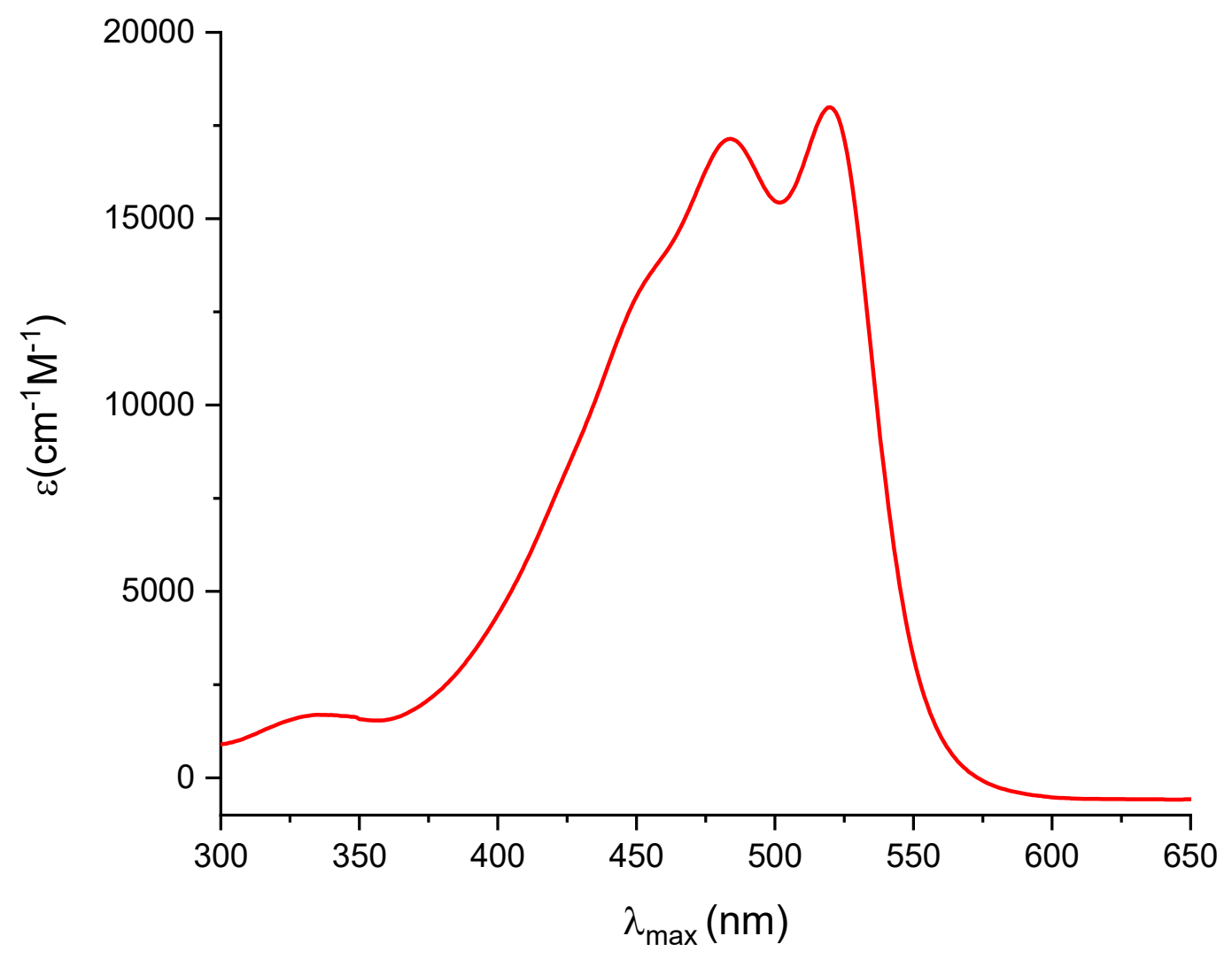

Figure S79. UV-Vis spectrum of $6(0.0566 \mathrm{mM})$ in $\mathrm{C}_{6} \mathrm{H}_{5} \mathrm{Cl}$. 


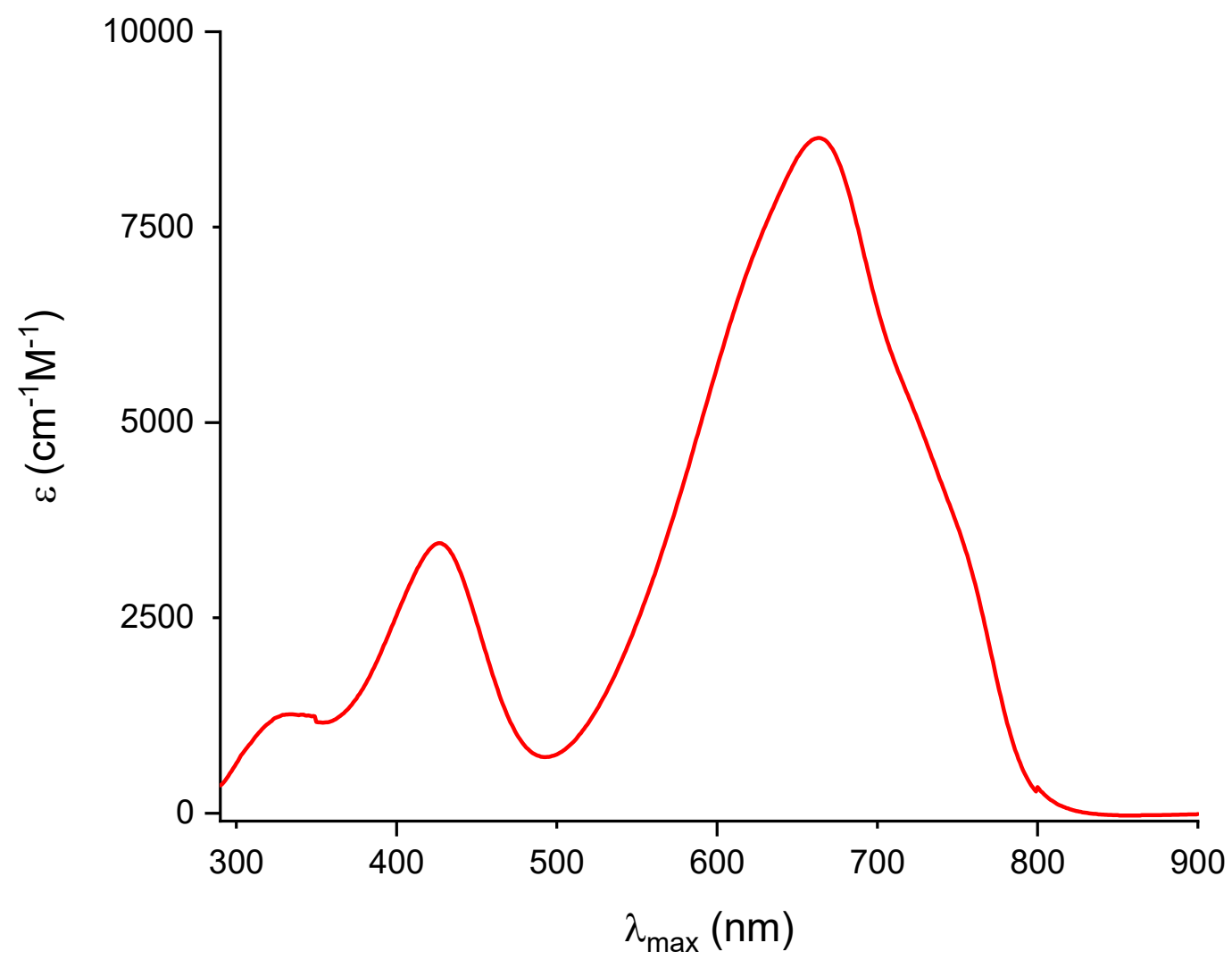

Figure S80. UV-Vis spectrum of $7(0.0385 \mathrm{mM})$ in $\mathrm{C}_{6} \mathrm{H}_{5} \mathrm{Cl}$. 


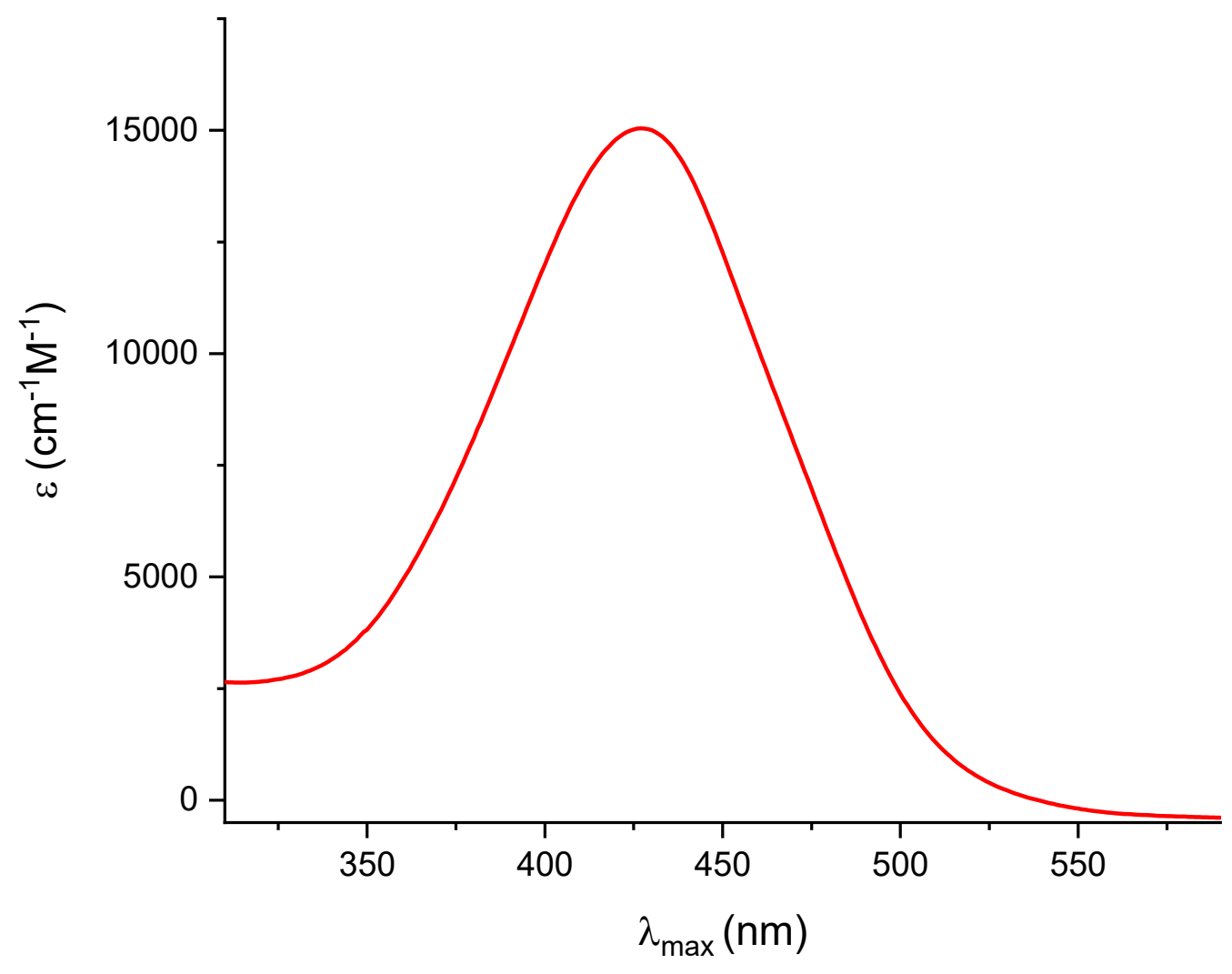

Figure S81. UV-Vis spectrum of $8(0.0135 \mathrm{mM})$ in $\mathrm{C}_{6} \mathrm{H}_{5} \mathrm{Cl}$. 


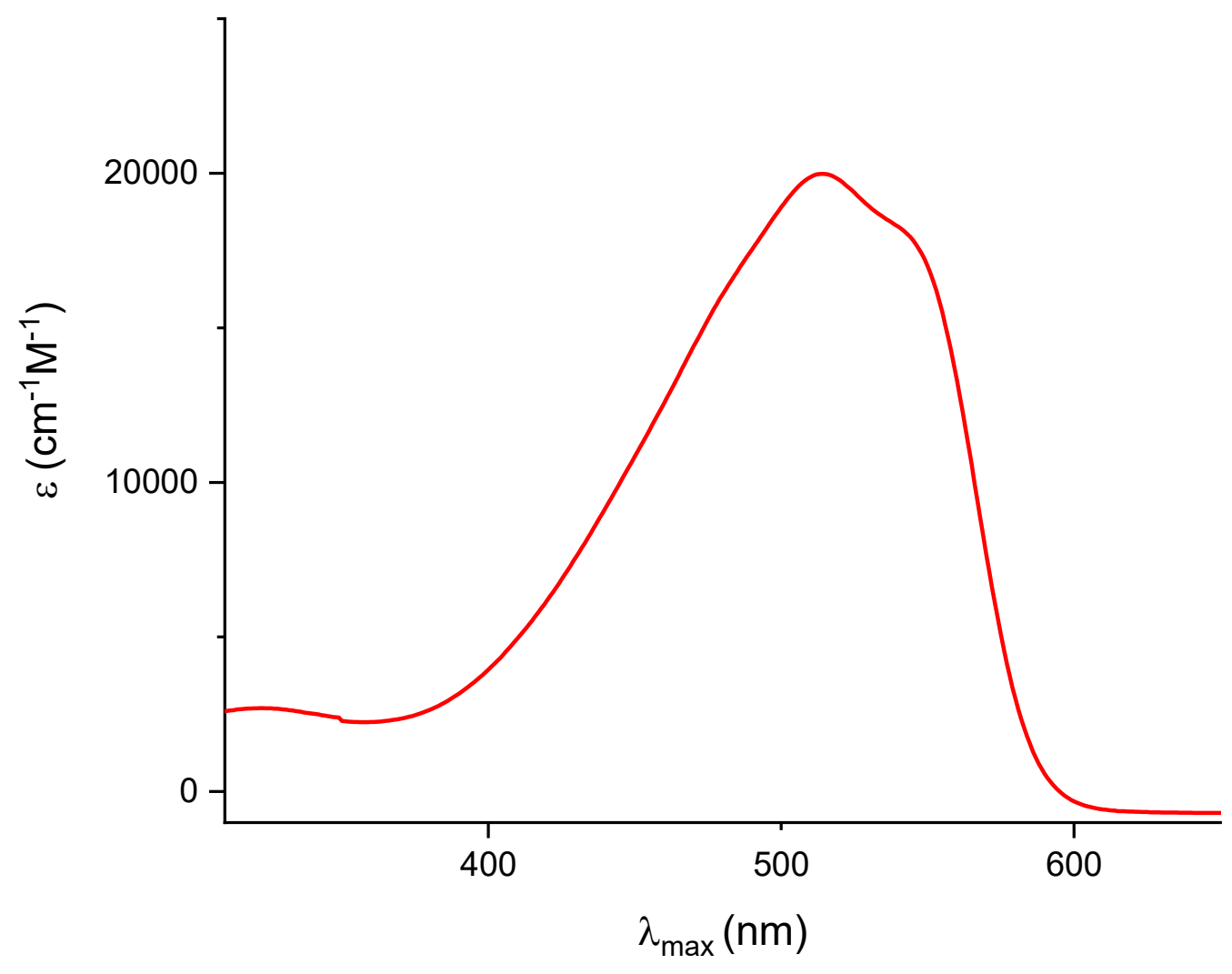

Figure S82. UV-Vis spectrum of $9(0.0456 \mathrm{mM})$ in $\mathrm{C}_{6} \mathrm{H}_{5} \mathrm{Cl}$. 


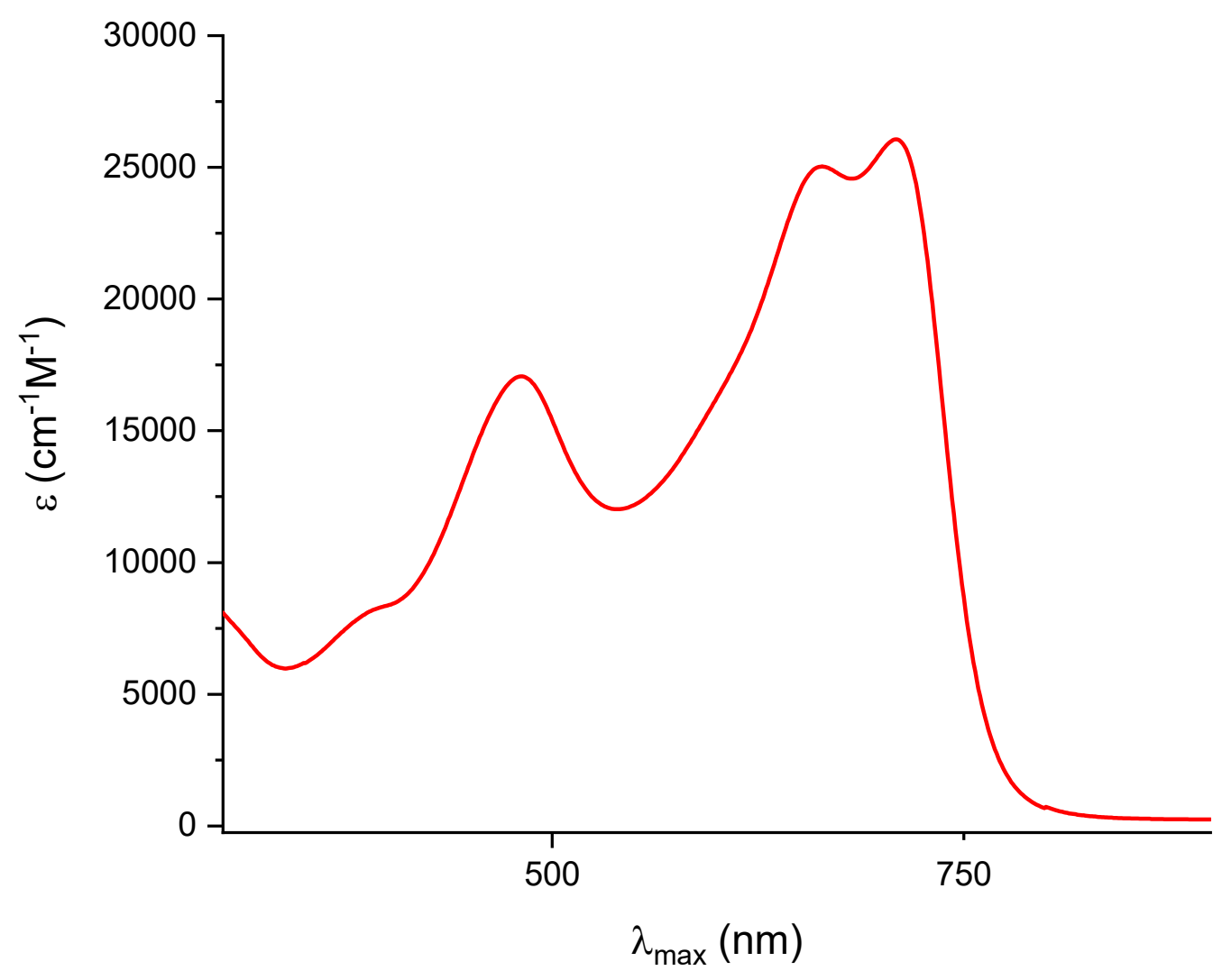

Figure S83. UV-Vis spectrum of $\mathbf{1 0}(0.0606 \mathrm{mM})$ in $\mathrm{C}_{6} \mathrm{H}_{5} \mathrm{Cl}$. 


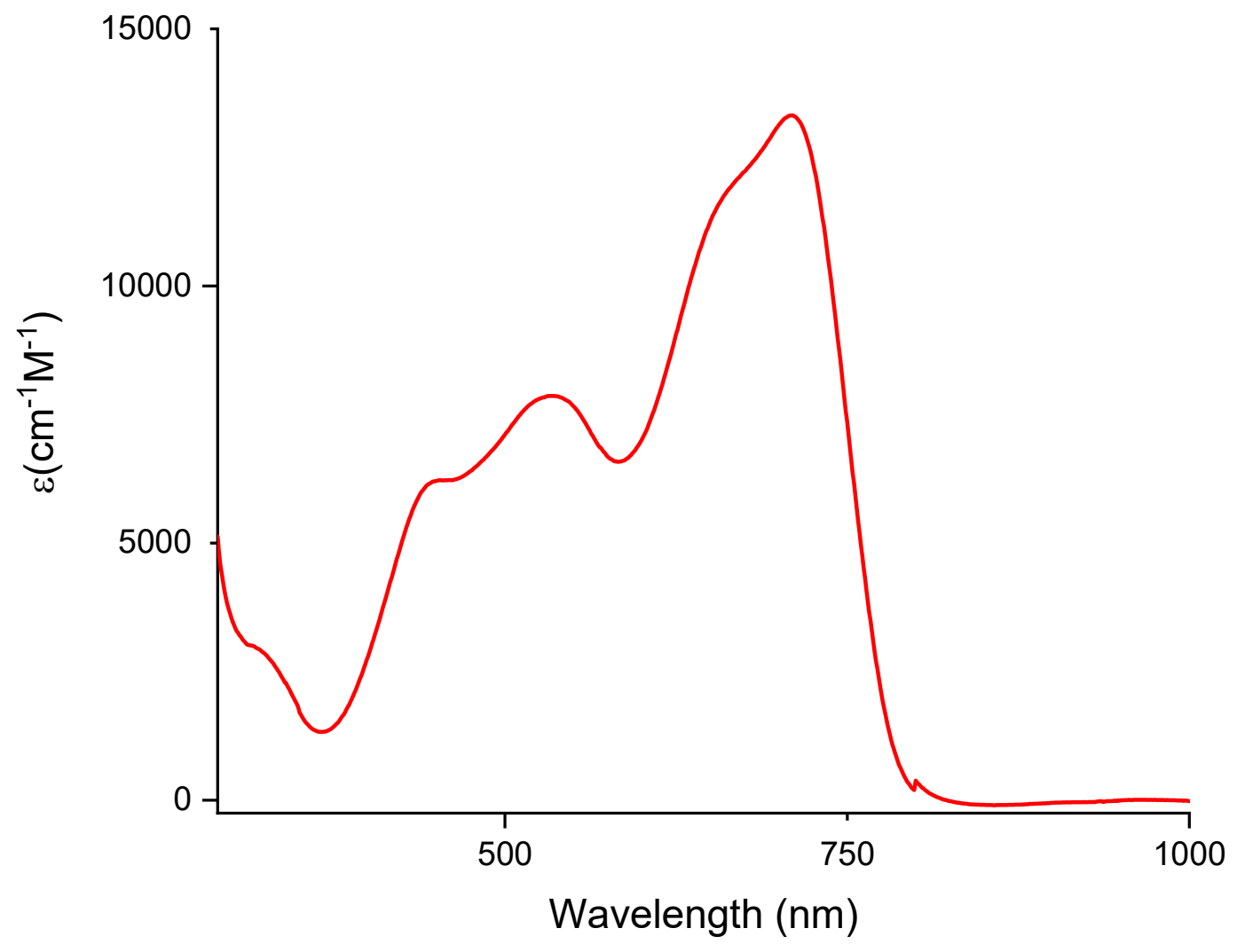

Figure S84. UV-Vis spectrum of $\mathbf{1 1}(0.0187 \mathrm{mM})$ in $\mathrm{C}_{6} \mathrm{H}_{5} \mathrm{Cl}$. 


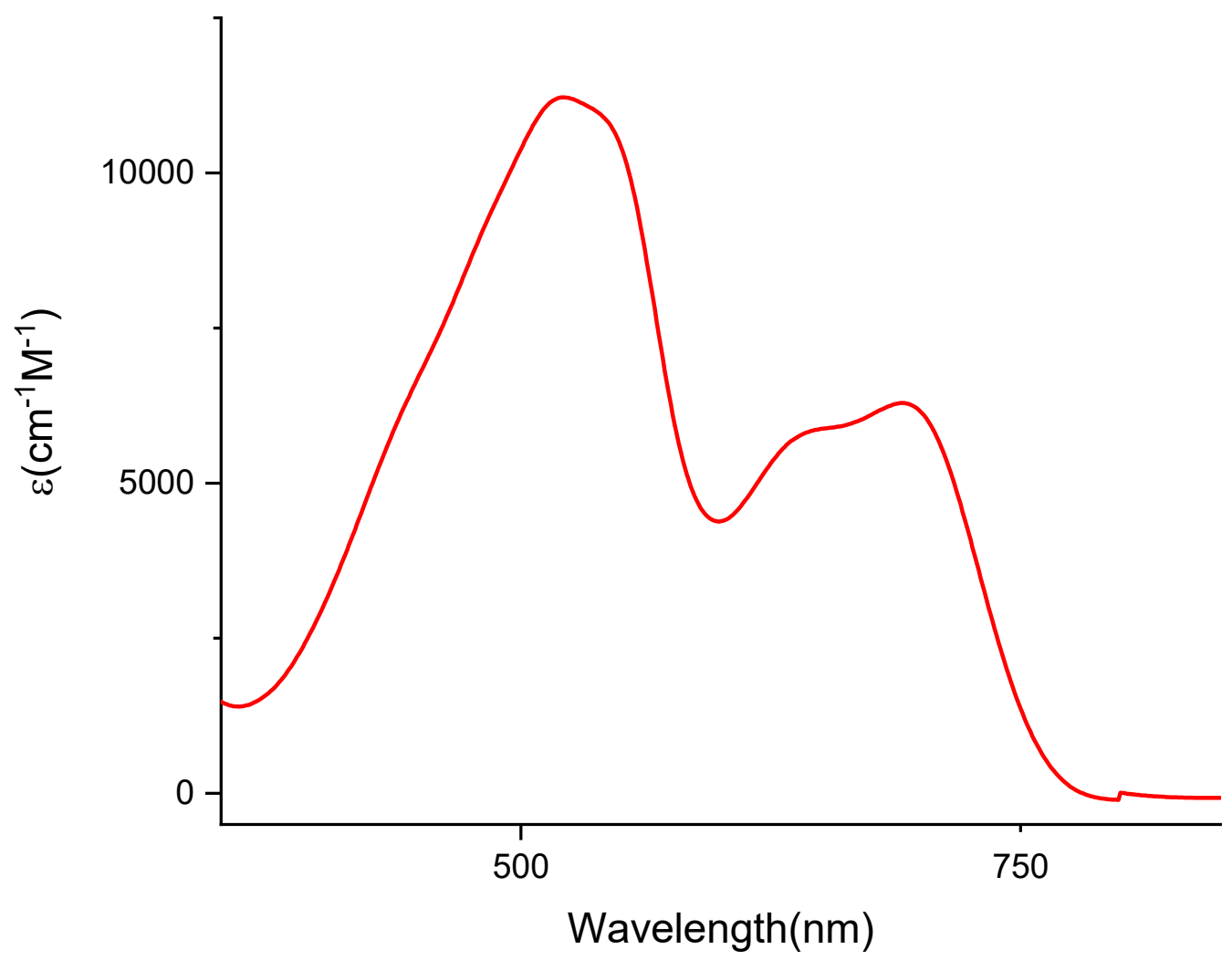

Figure S85. UV-Vis spectrum of $\mathbf{1 2}(0.0351 \mathrm{mM})$ in $\mathrm{C}_{6} \mathrm{H}_{5} \mathrm{Cl}$. 


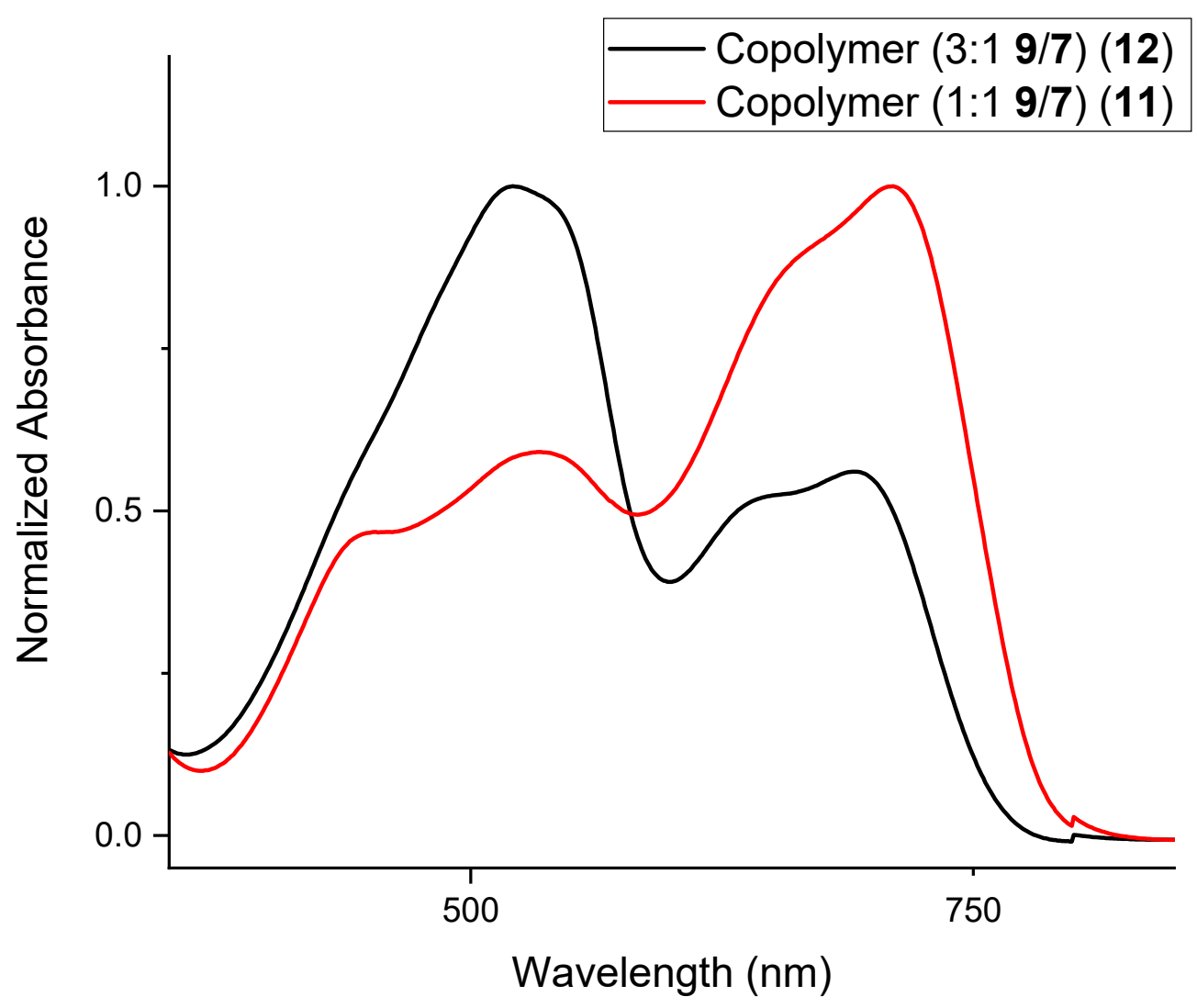

Figure S86. UV-Vis spectrum of $\mathbf{1 1}$ and 12 overlayed in $\mathrm{C}_{6} \mathrm{H}_{5} \mathrm{Cl}$. 


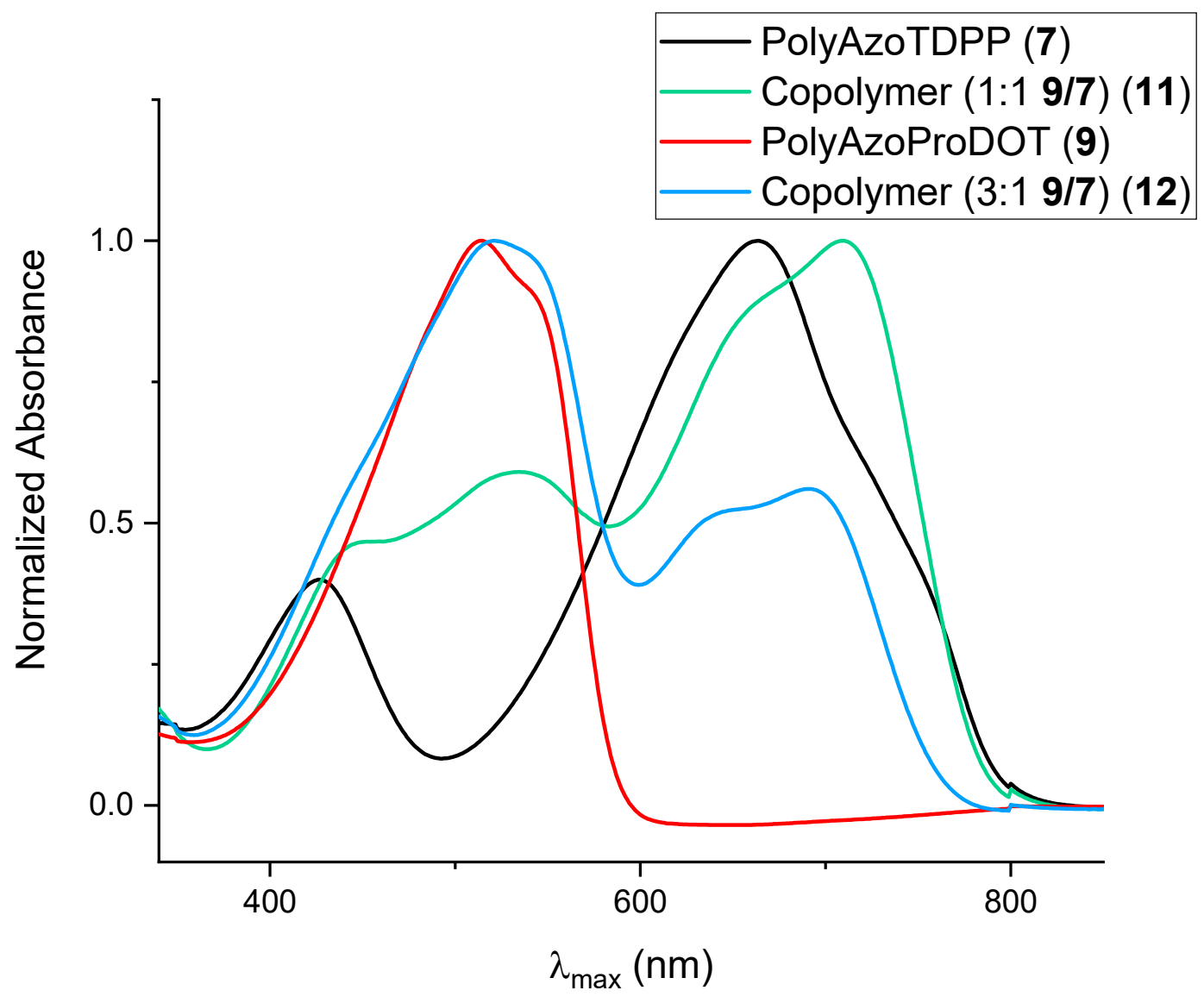

Figure S87. UV-Vis spectrum of 7, 9, 11 and 12 overlayed in $\mathrm{C}_{6} \mathrm{H}_{5} \mathrm{Cl}$. 


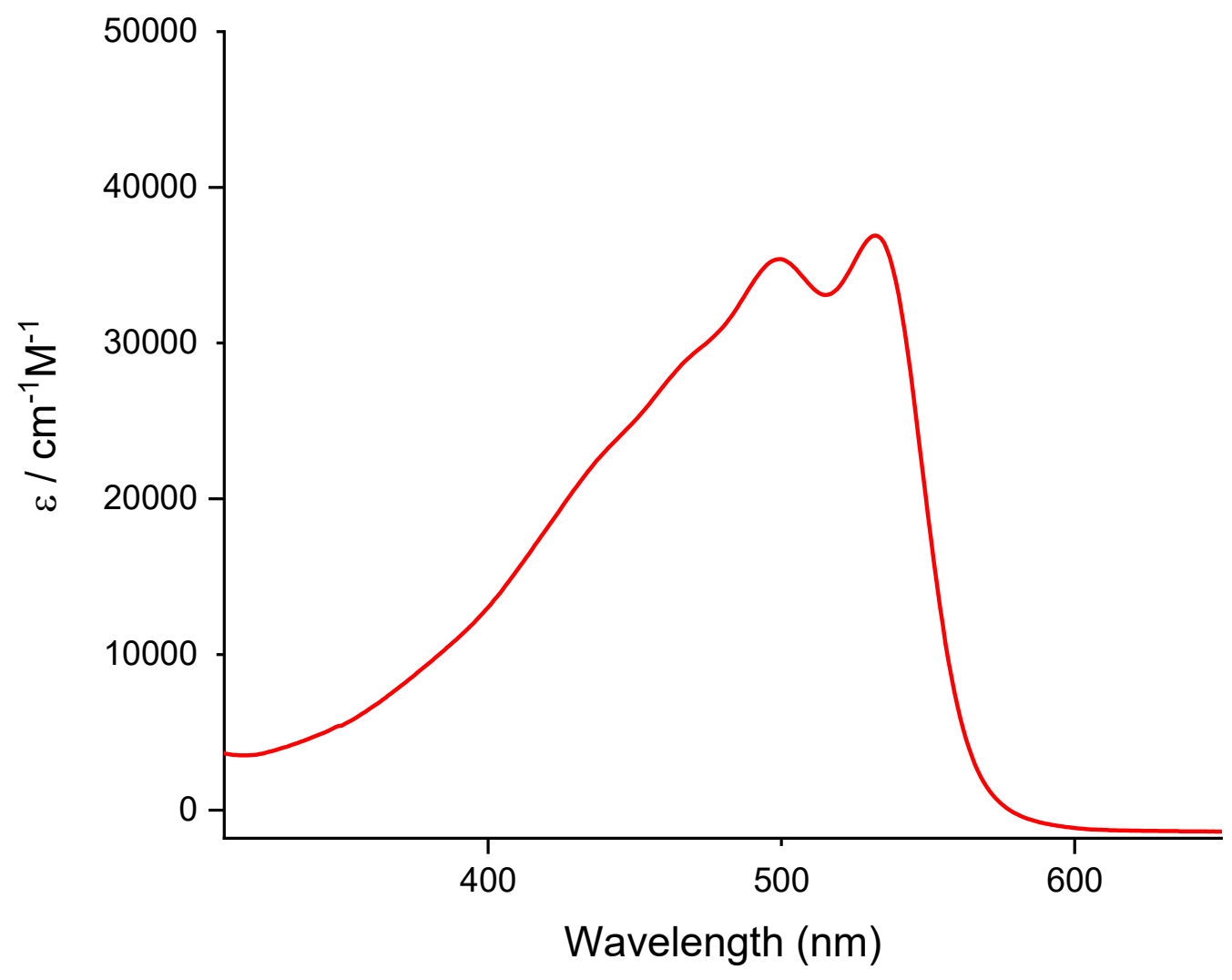

Figure S88. UV-Vis spectrum of $\mathbf{1 3}(0.0428 \mathrm{mM})$ in $\mathrm{C}_{6} \mathrm{H}_{5} \mathrm{Cl}$. 


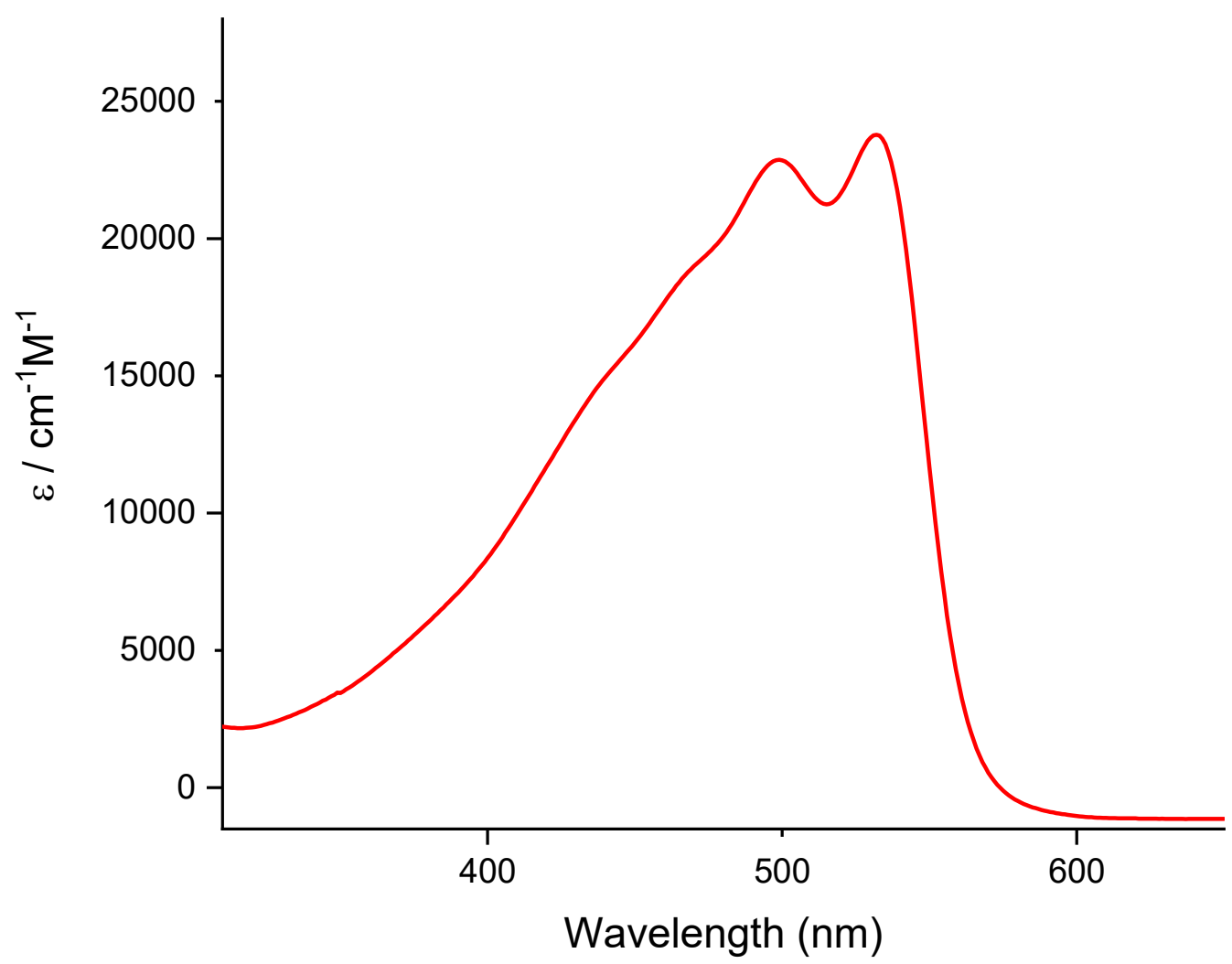

Figure S89. UV-Vis spectrum of $14(0.0428 \mathrm{mM})$ in $\mathrm{C}_{6} \mathrm{H}_{5} \mathrm{Cl}$. 


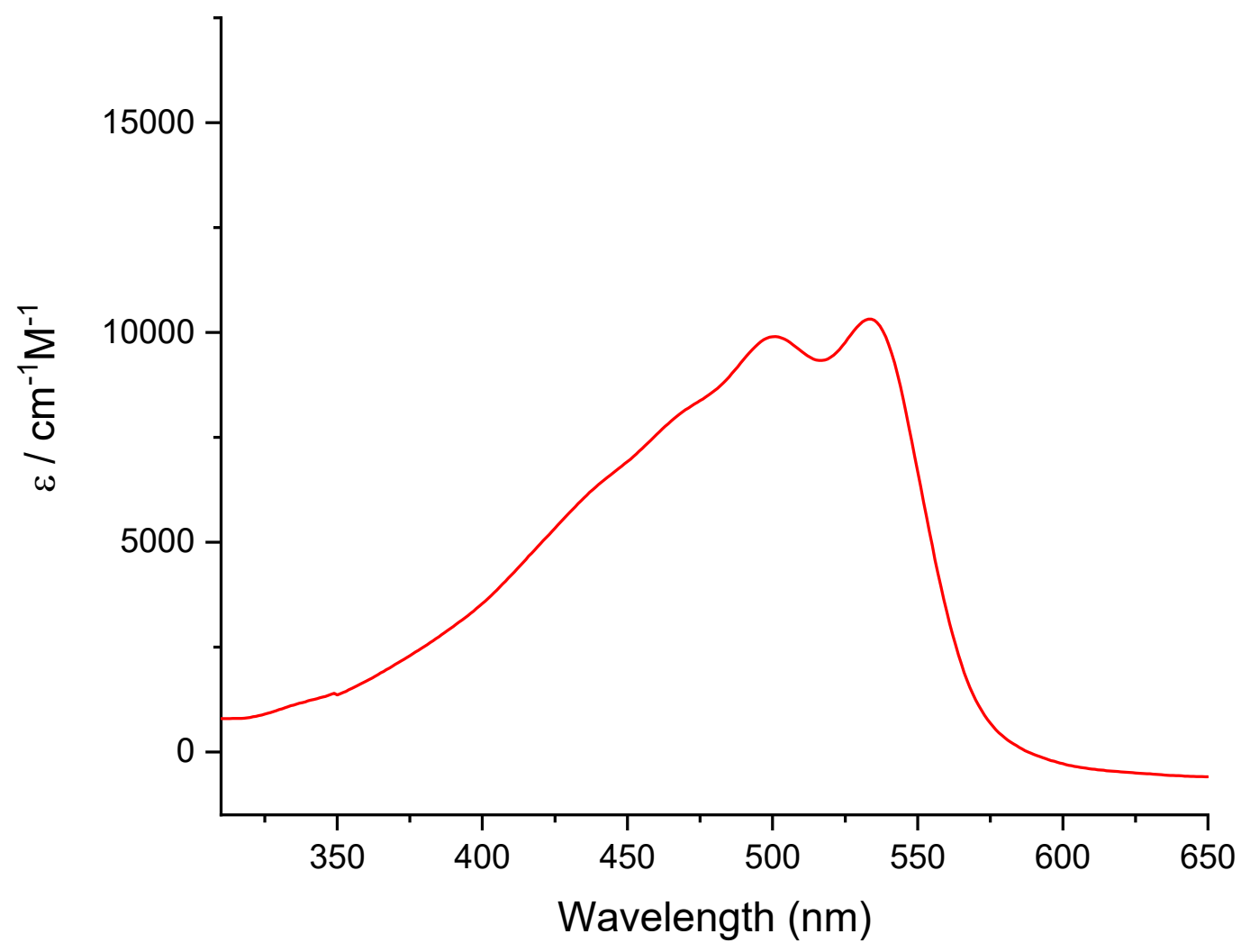

Figure S90. UV-Vis spectrum of $15(0.0551 \mathrm{mM})$ in $\mathrm{C}_{6} \mathrm{H}_{5} \mathrm{Cl}$. 


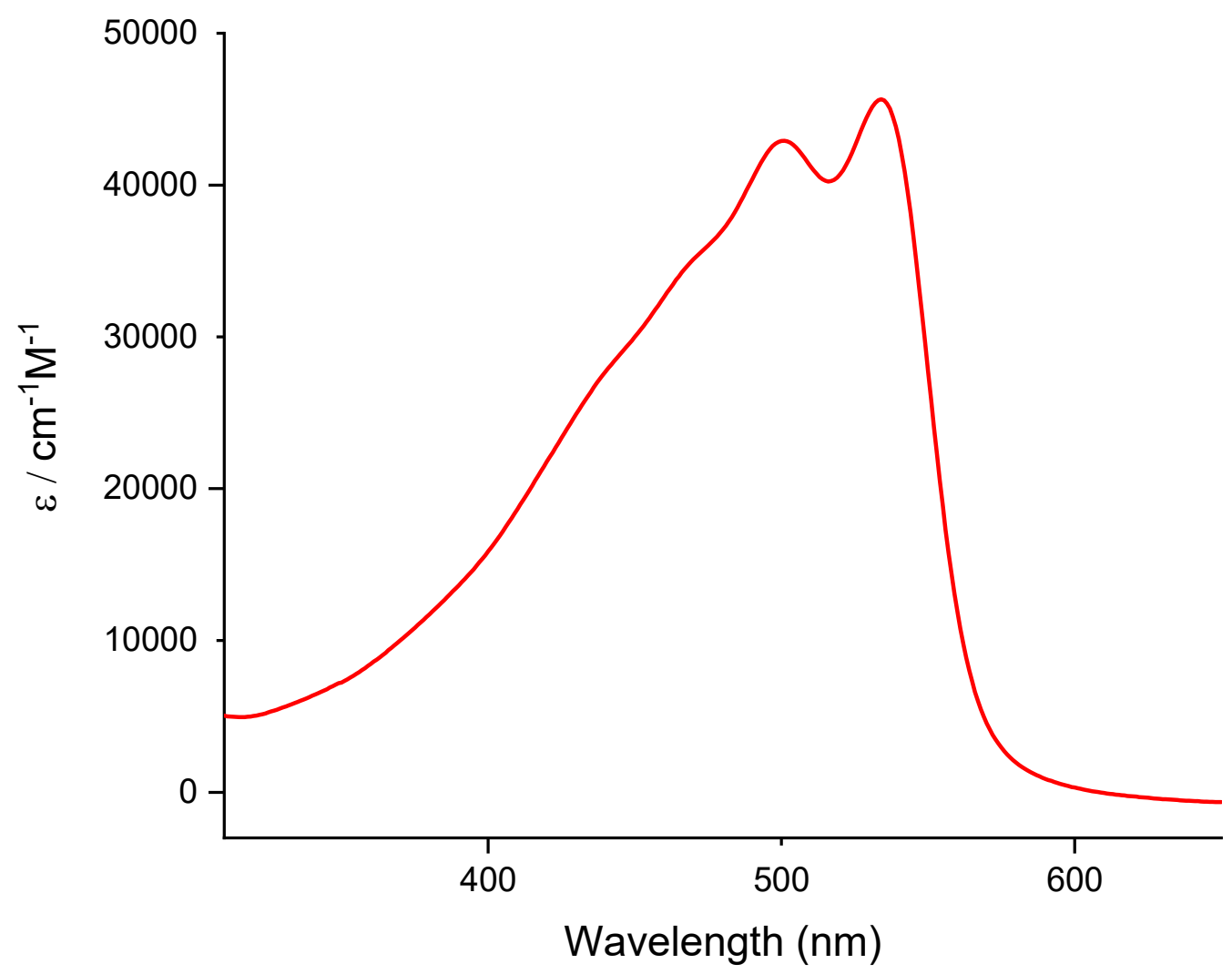

Figure S91. UV-Vis spectrum of $\mathbf{1 6}(0.0612 \mathrm{mM})$ in $\mathrm{C}_{6} \mathrm{H}_{5} \mathrm{Cl}$. 


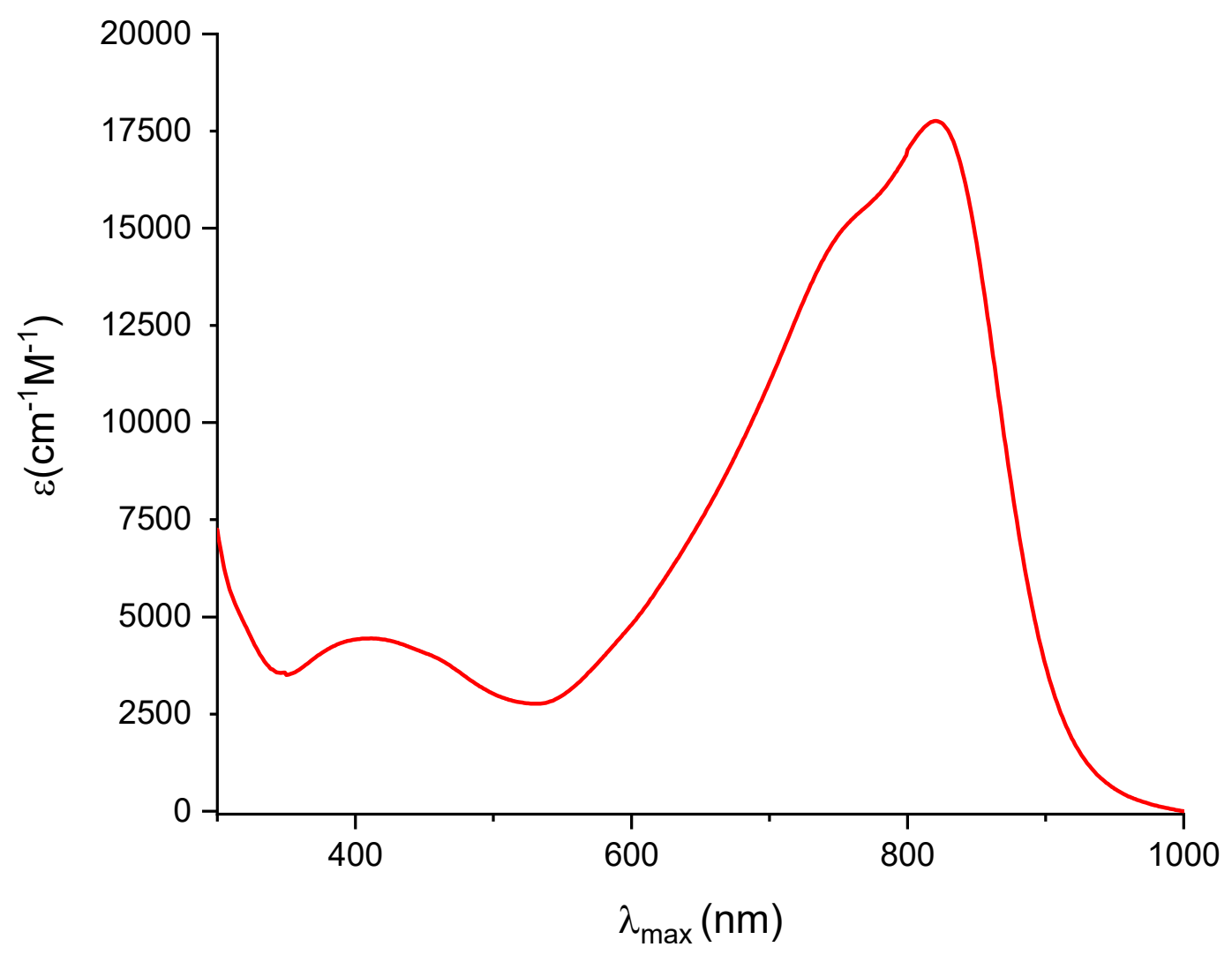

Figure S92. UV-Vis spectrum of $18(0.0326 \mathrm{mM})$ in $\mathrm{C}_{6} \mathrm{H}_{5} \mathrm{Cl}$. 


\section{IR Spectra}

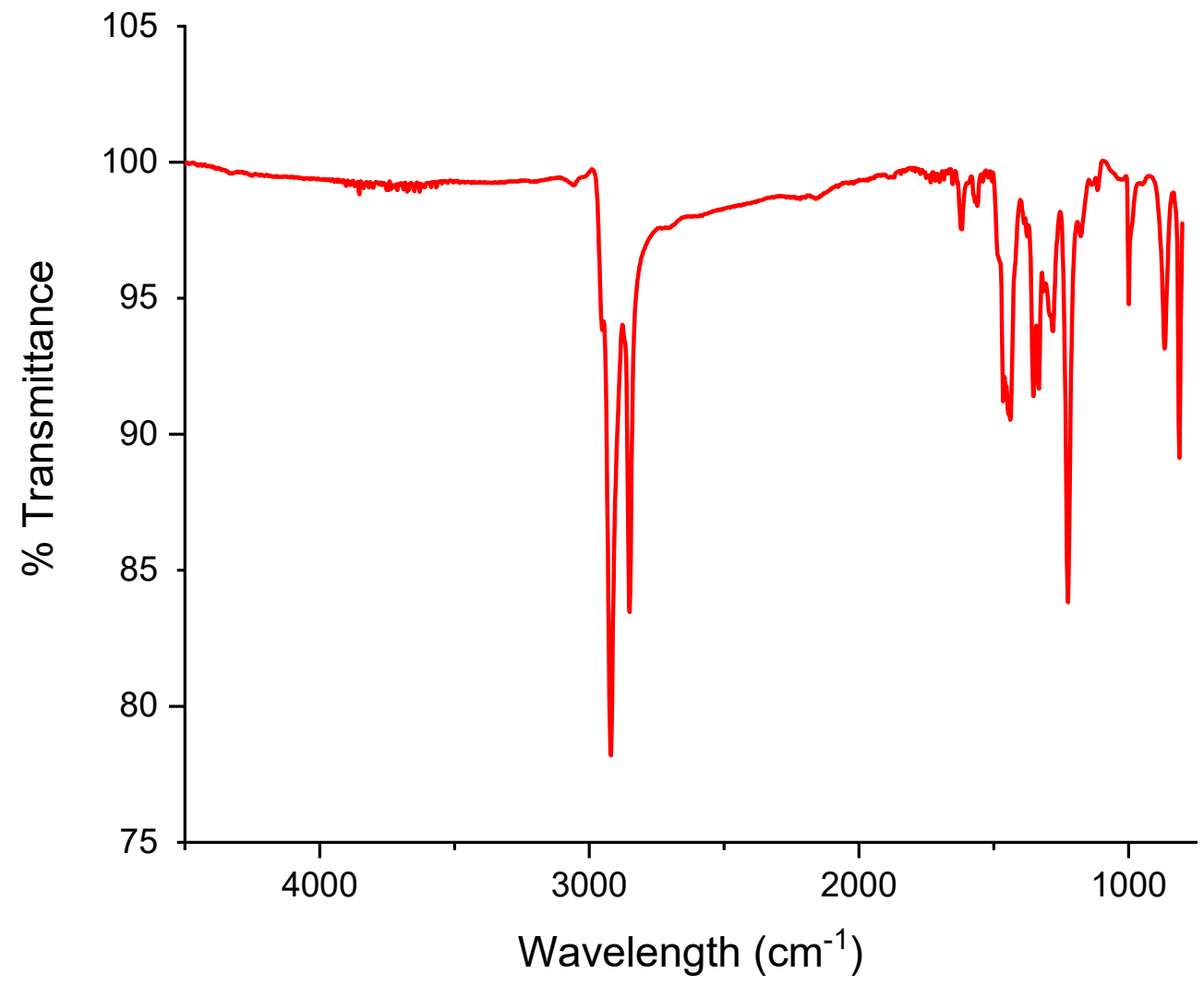

Figure S93. FT-IR spectrum of 3. 


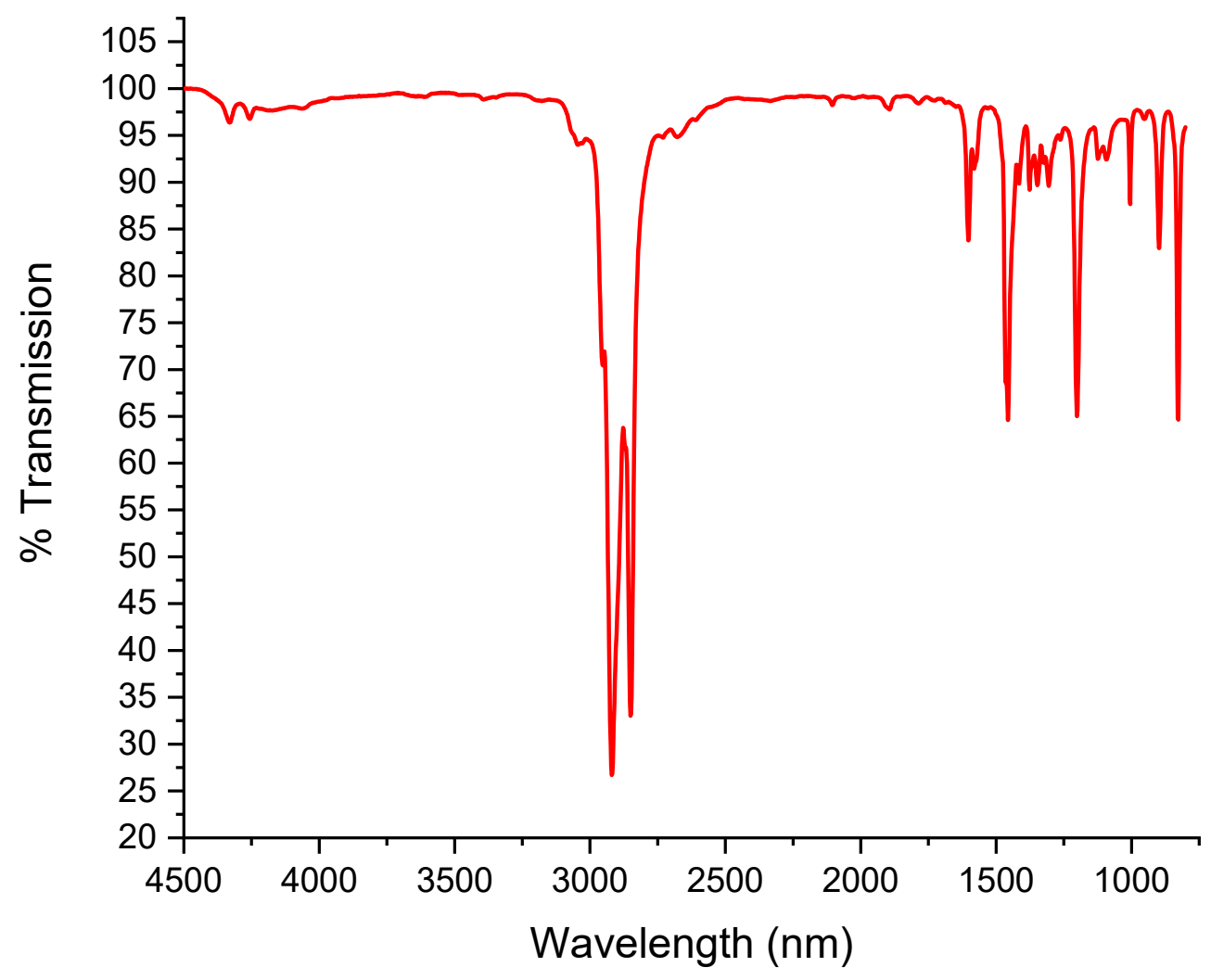

Figure S94. FT-IR spectrum of 6. 


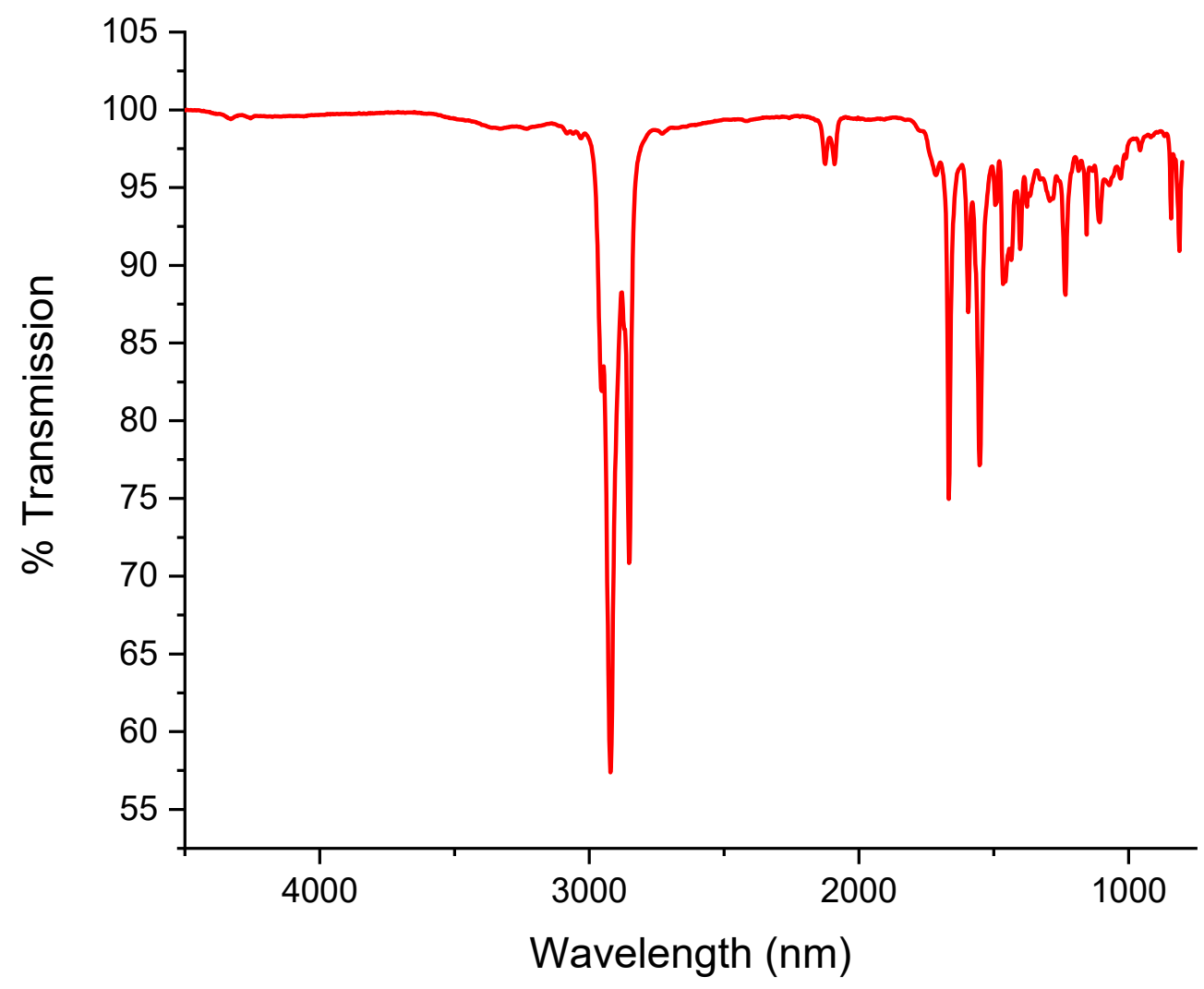

Figure S95. FT-IR spectrum of 7. 


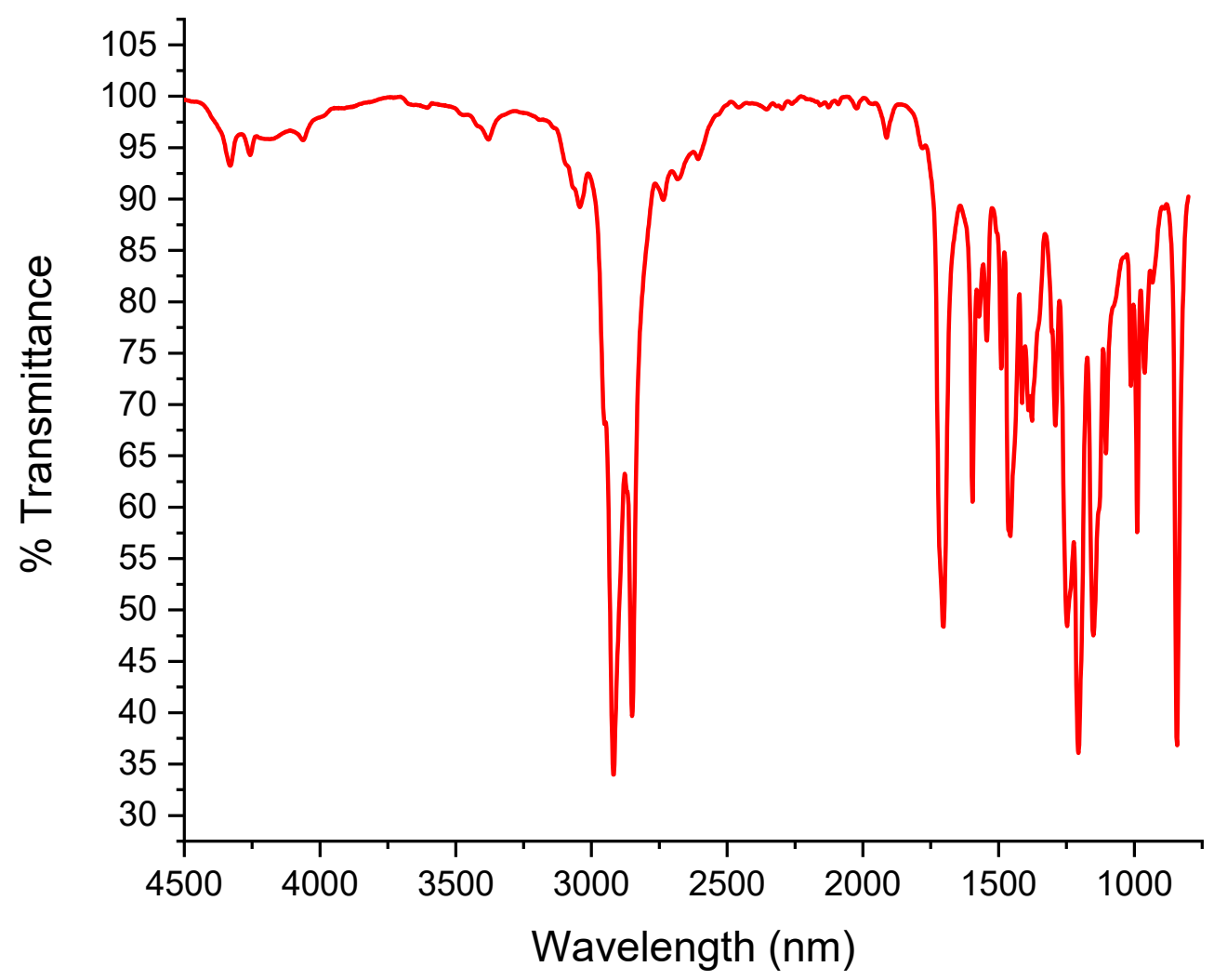

Figure S96. FT-IR spectrum of 8. 


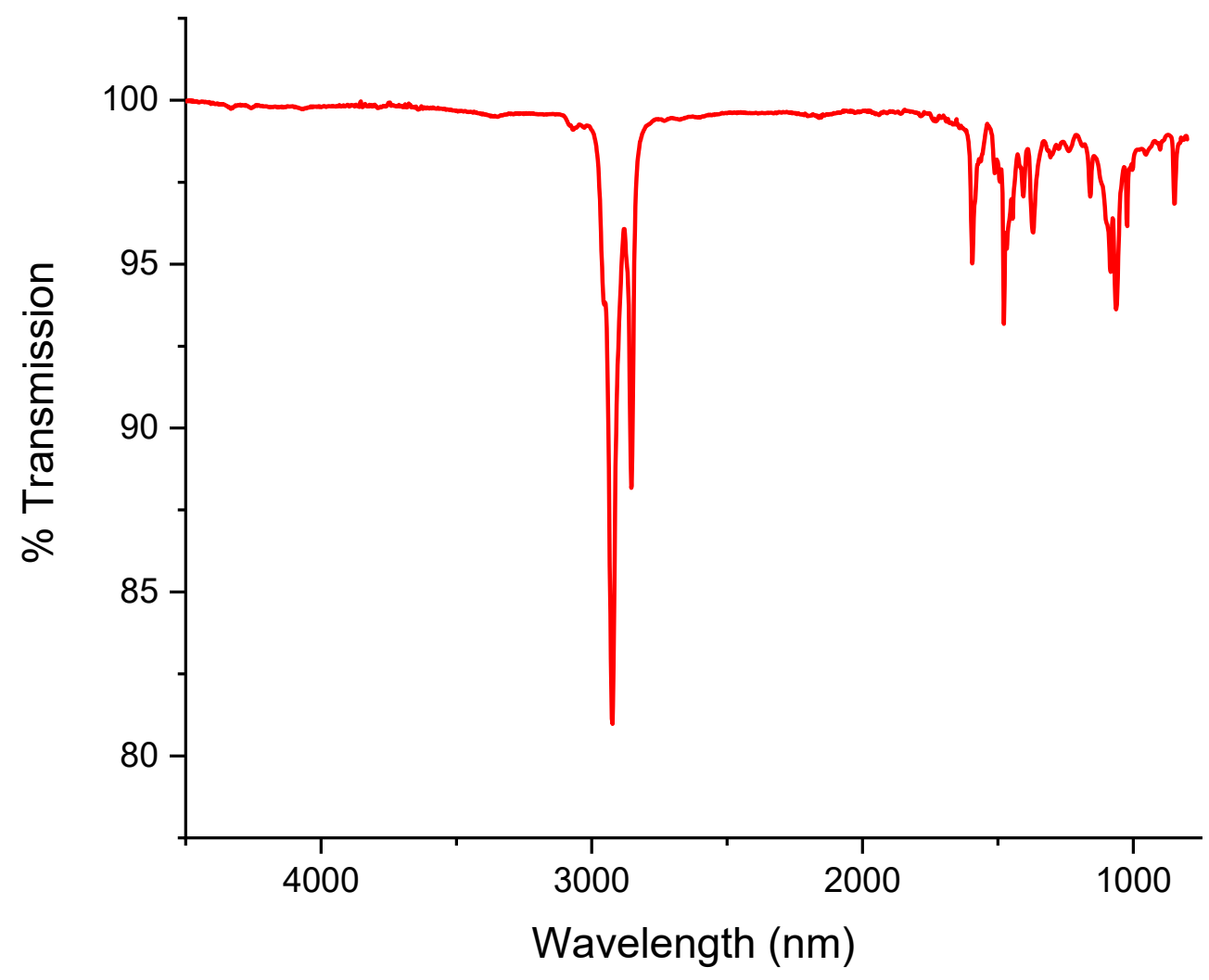

Figure S97. FT-IR spectrum of 9. 


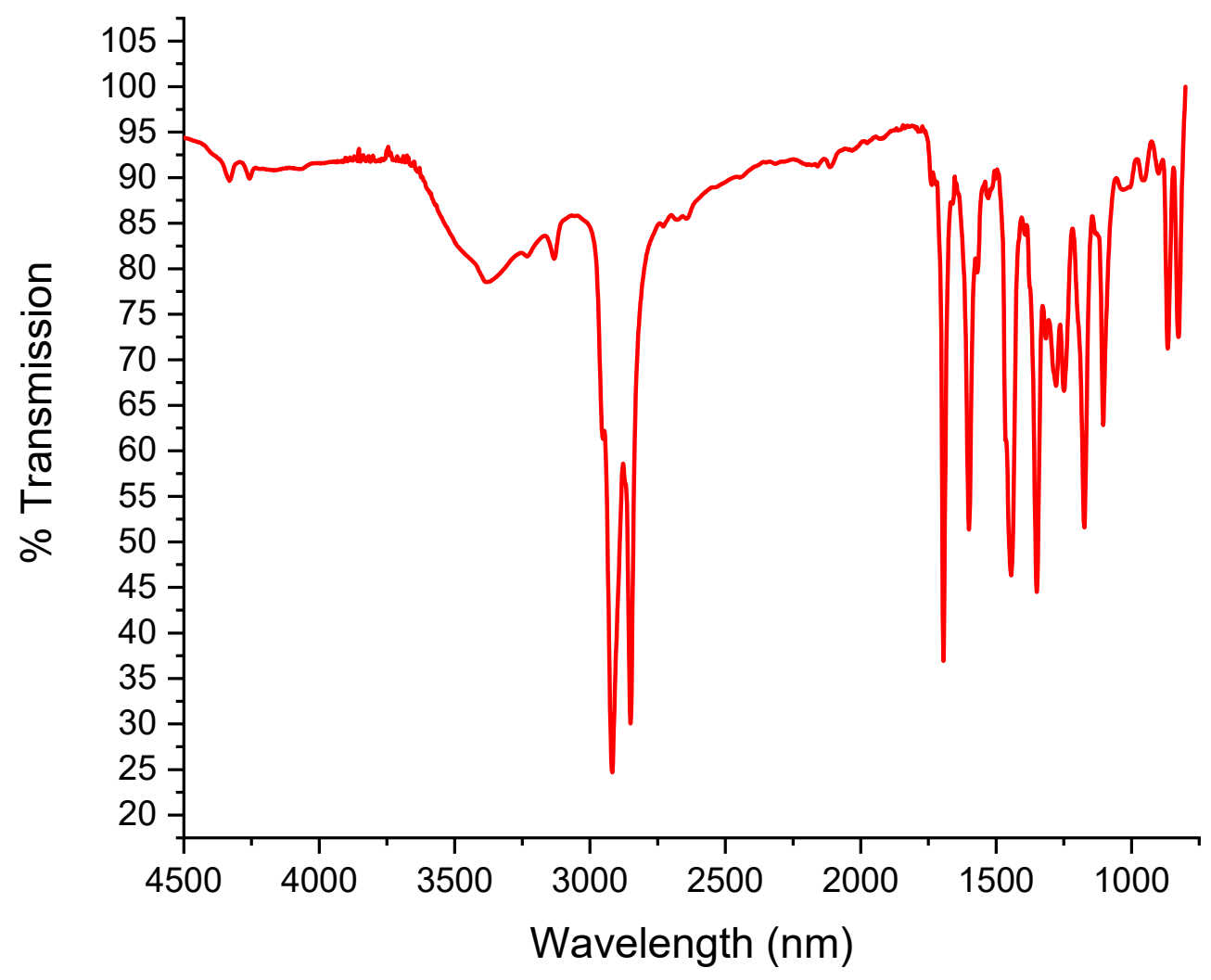

Figure S98. FT-IR spectrum of $\mathbf{1 0 .}$ 


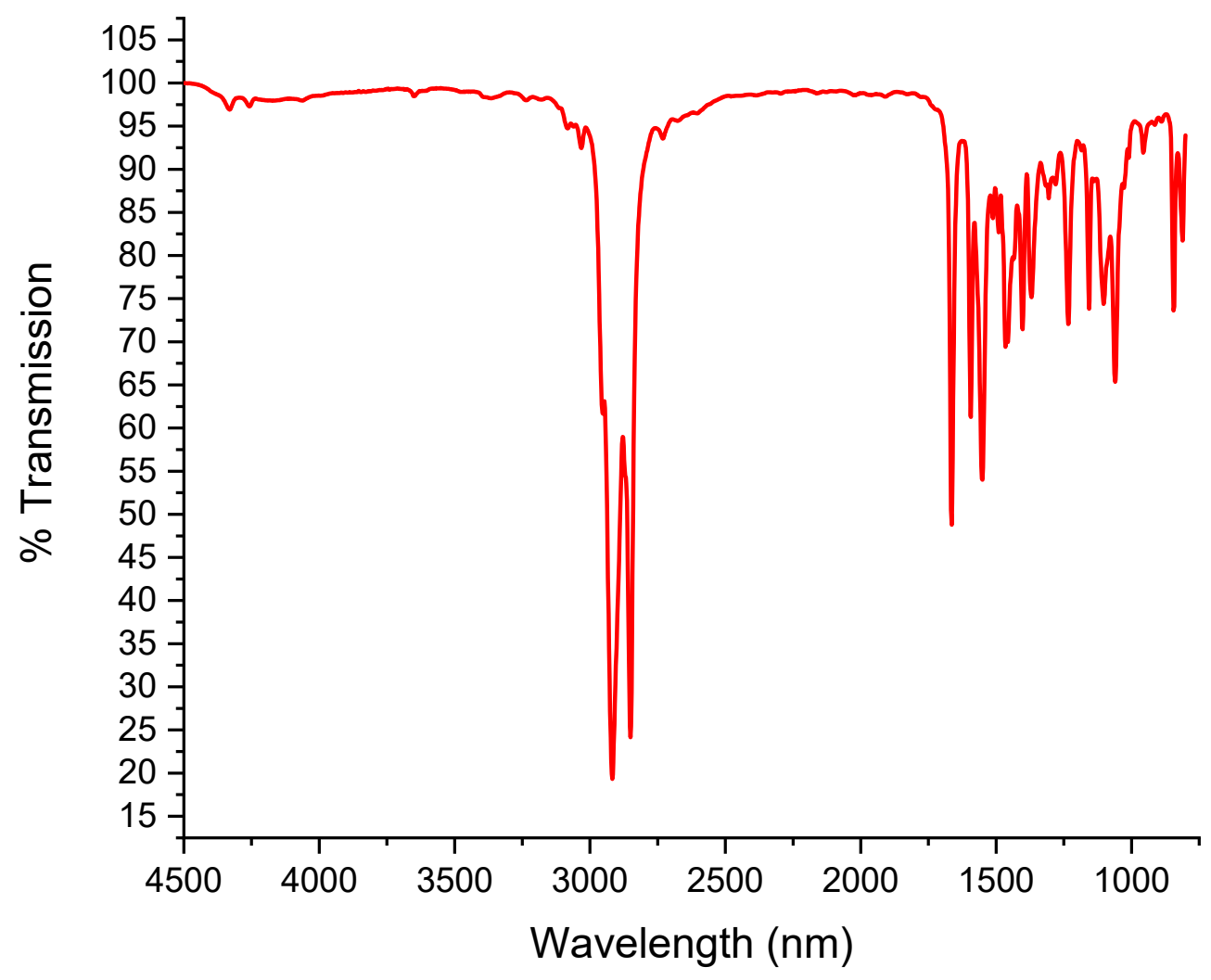

Figure S99. FT-IR spectrum of 11. 


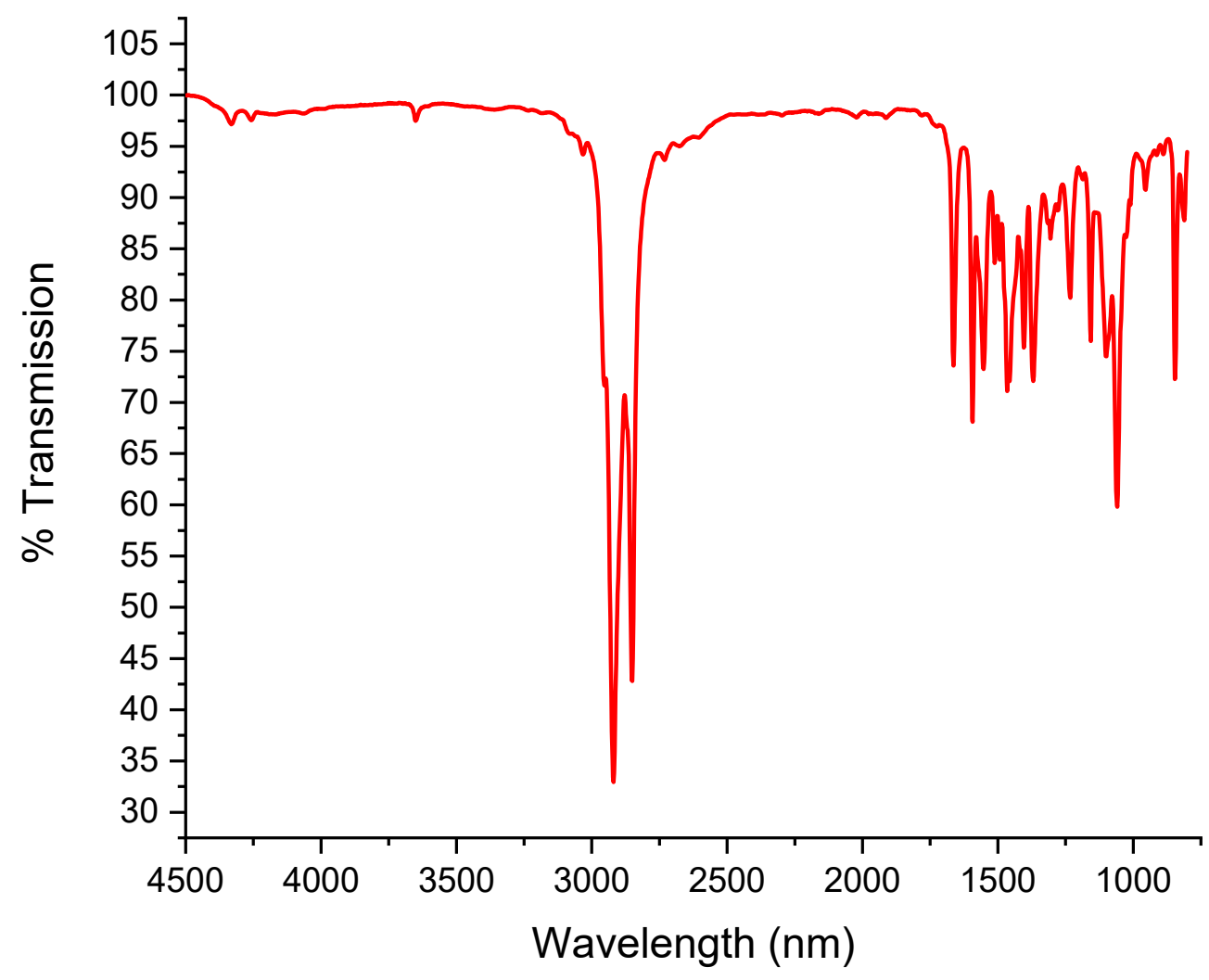

Figure S100. FT-IR spectrum of 12. 


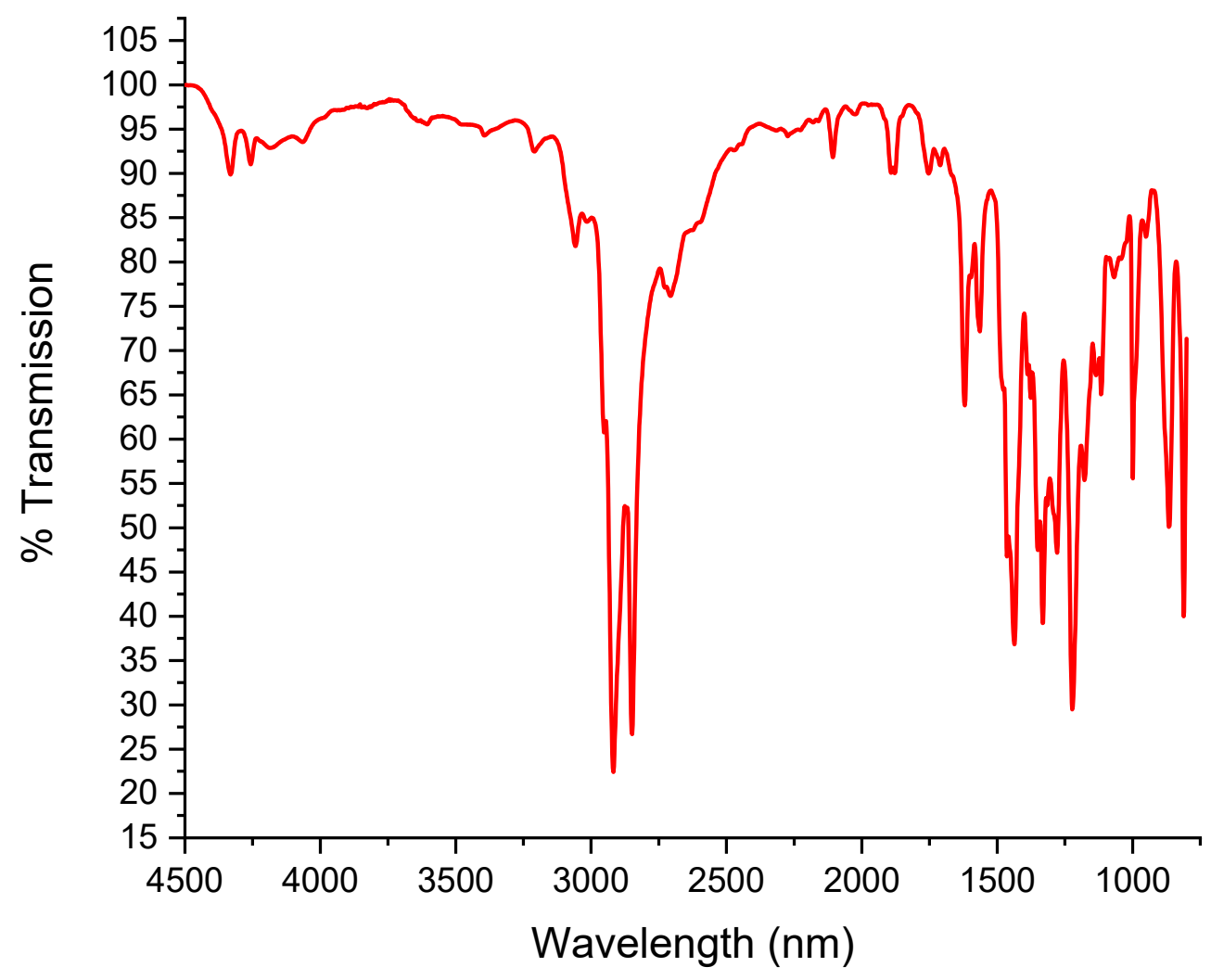

Figure S101. FT-IR spectrum of 13. 


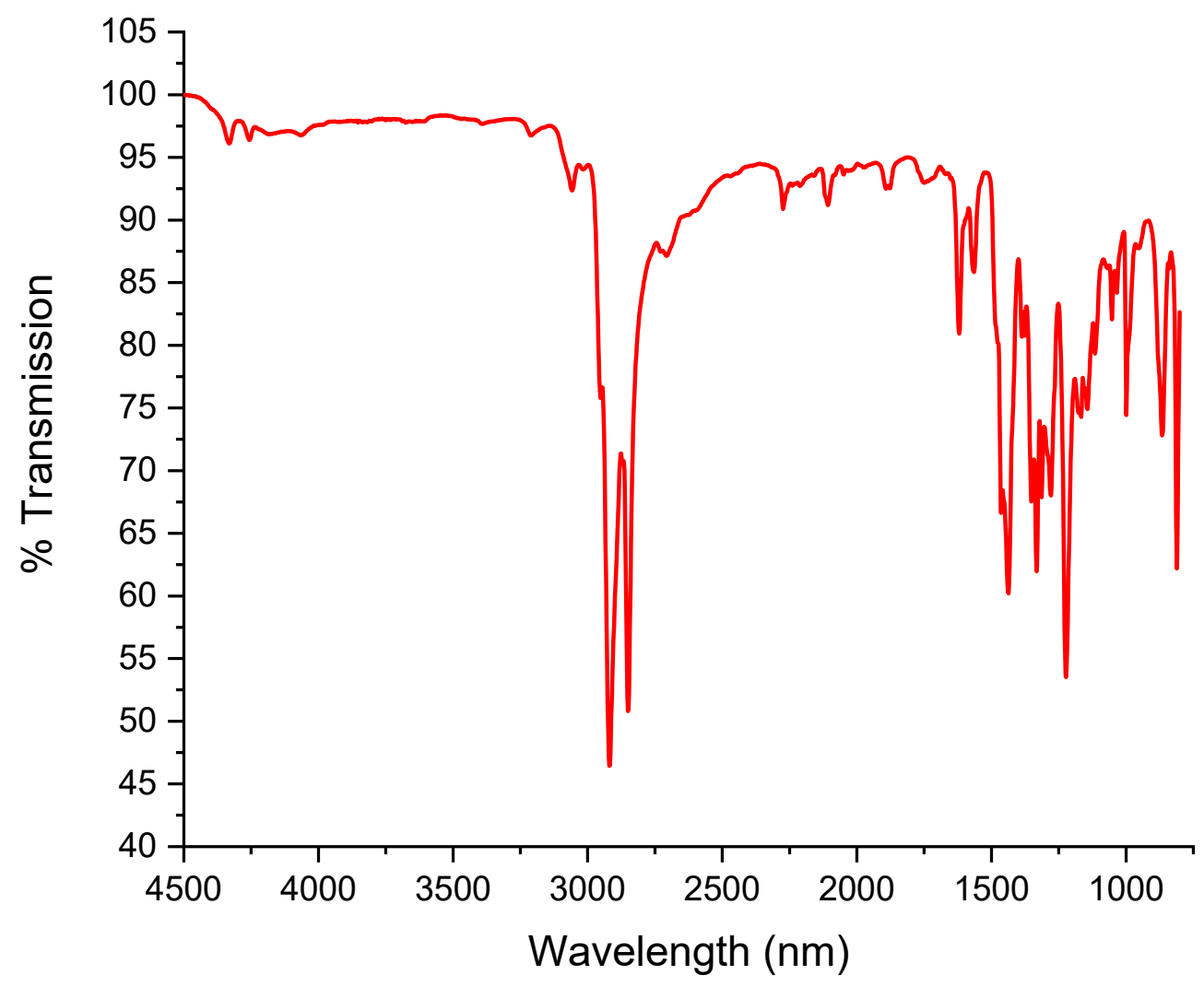

Figure S102. FT-IR spectrum of 14. 


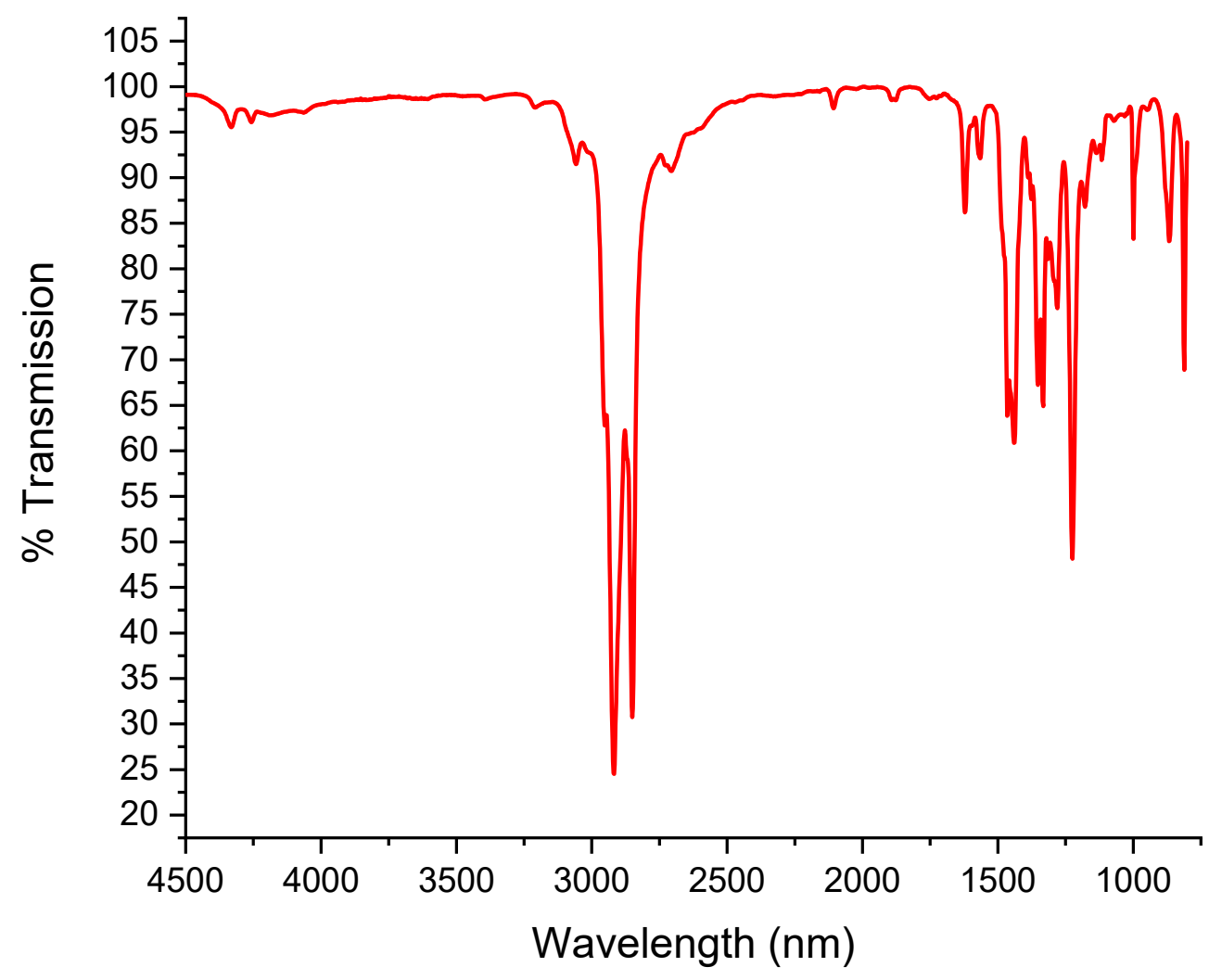

Figure S103. FT-IR spectrum of 15. 
Supporting Information

$\$ 130$

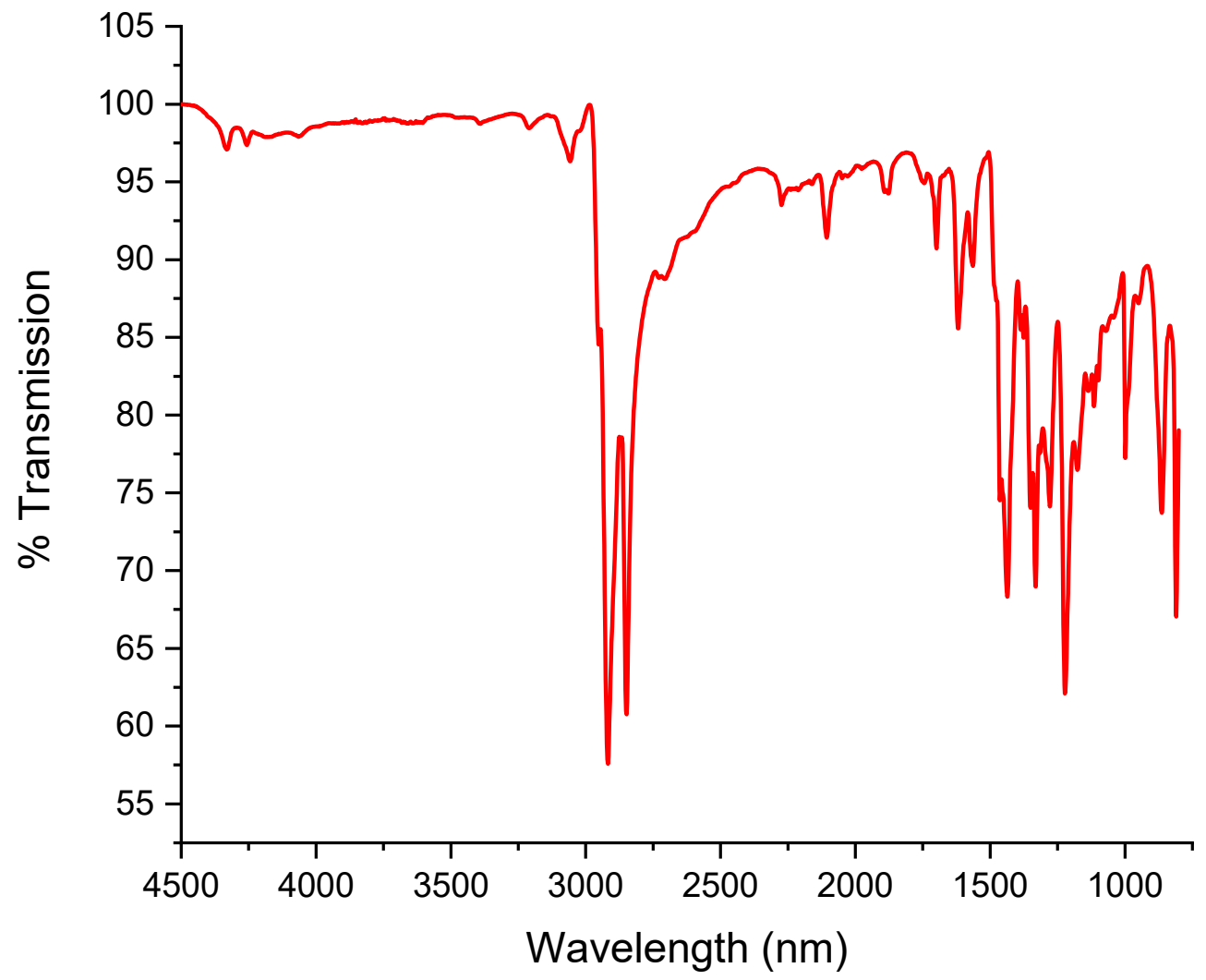

Figure S104. FT-IR spectrum of 16. 


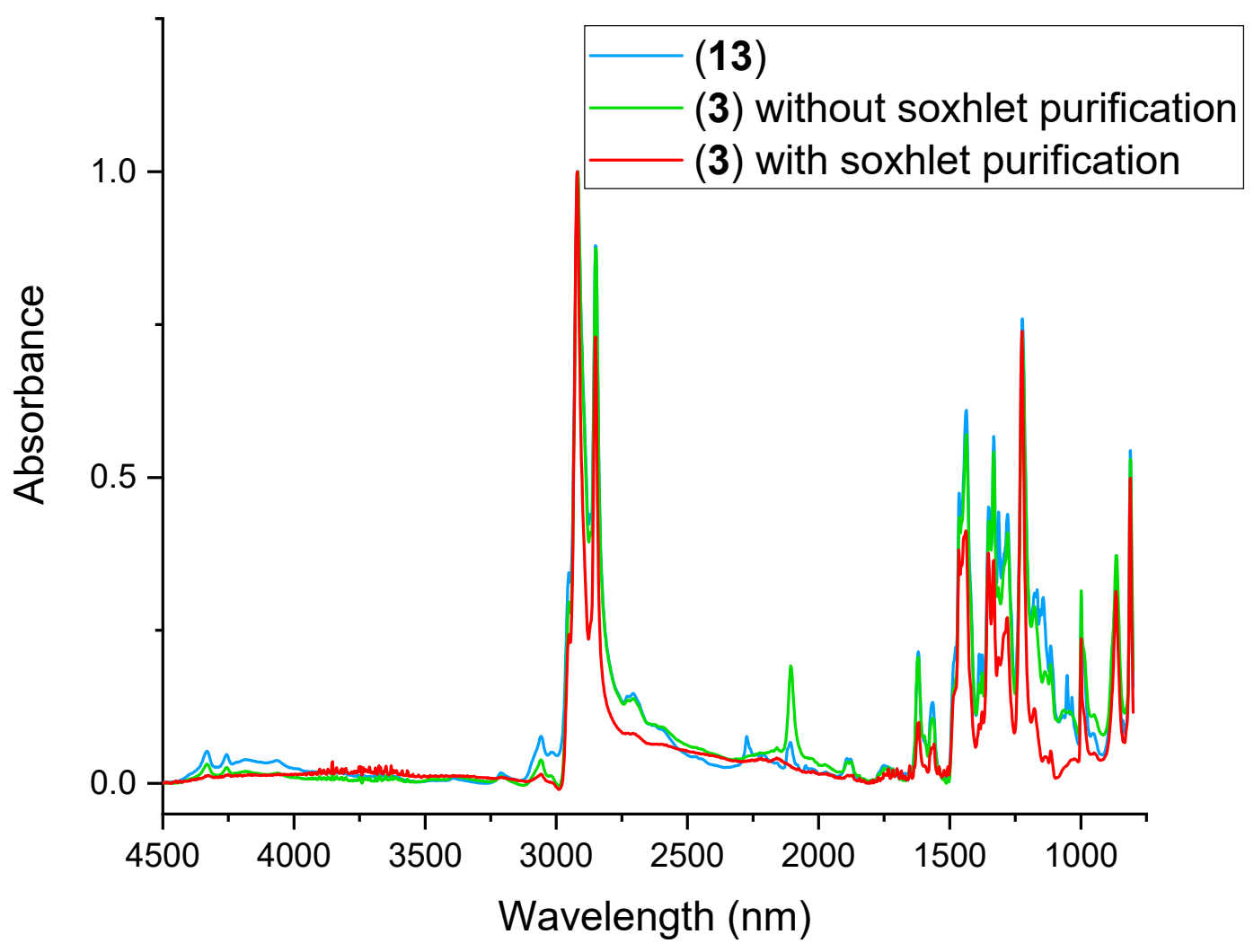

Figure S105. Normalized FT-IR spectrum of 3 (soxhlet purified), 3 (without soxhlet purification) and 13. 


\section{High Temperature Gel Permeation Chromotography (HT-GPC) Data}

$[\mathrm{mV}]$

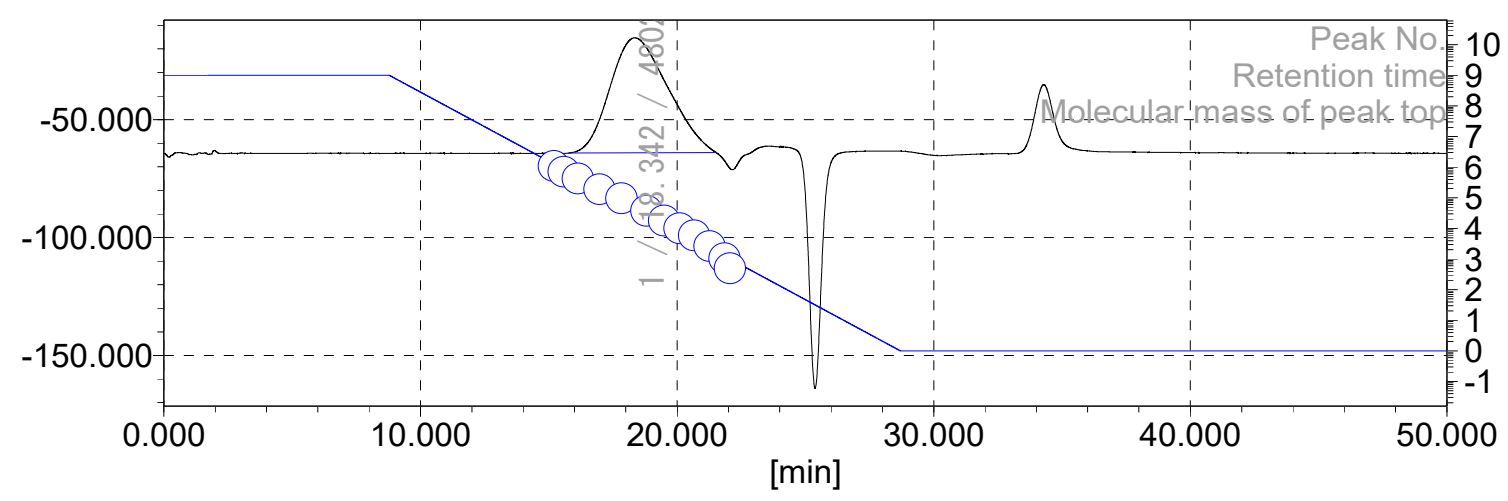

\begin{tabular}{|c|c|c|c|c|c|}
\hline & {$[\mathrm{min}]$} & {$[\mathrm{mV}]$} & {$[\mathrm{mol}]$} & Mn & 20,466 \\
\hline Peak start & 15.795 & -64.144 & 680,035 & Mw & 58,715 \\
\hline Peak top & 18.342 & -15.291 & 48,021 & $\mathrm{Mz}$ & 124,946 \\
\hline \multirow[t]{2}{*}{ Peak end } & 21.522 & -63.993 & 1,754 & $M z+1$ & 203,599 \\
\hline & & & & Mv & 58,715 \\
\hline Height [mV] & & & 48.786 & $\mathrm{Mp}$ & 48,021 \\
\hline Area $\left[\mathrm{mV}^{*} \mathrm{~s}\right]$ & & & 7608.918 & $\mathrm{Mz} / \mathrm{Mw}$ & 2.128 \\
\hline Area\% [\%] & & & 100.000 & $\mathrm{Mw} / \mathrm{Mn}$ & 2.869 \\
\hline [eta] & & & 58715.20917 & $\mathrm{Mz}+1 / \mathrm{Mw}$ & 3.468 \\
\hline
\end{tabular}

Figure S106. HT-GPC data for optimization of 3 (entry 1).

$[\mathrm{mV}]$

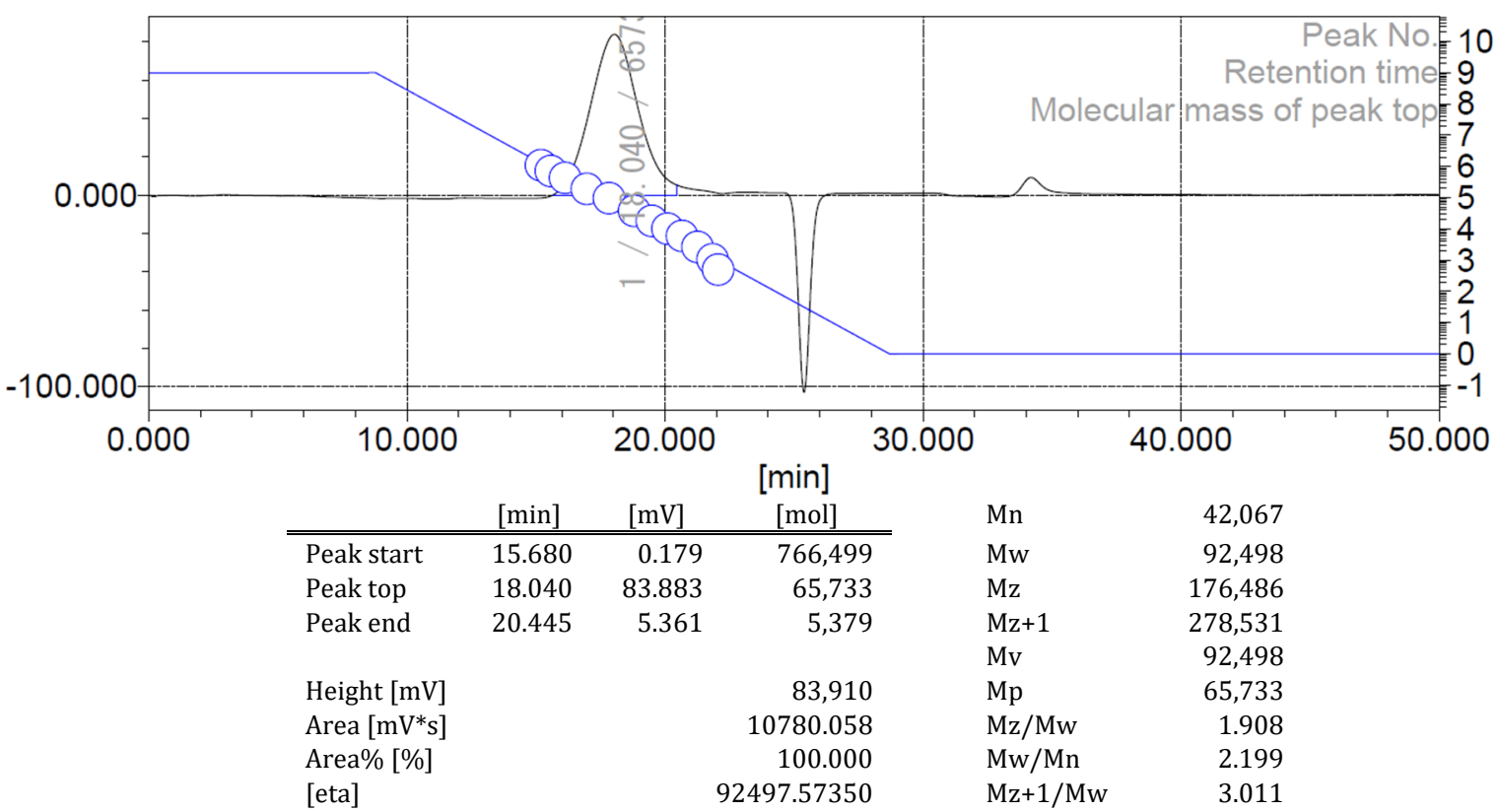

[LogM]

Figure S107. HT-GPC data for optimization of $\mathbf{3}$ (entry 2). 
$[\mathrm{mV}]$

$[\log M]$

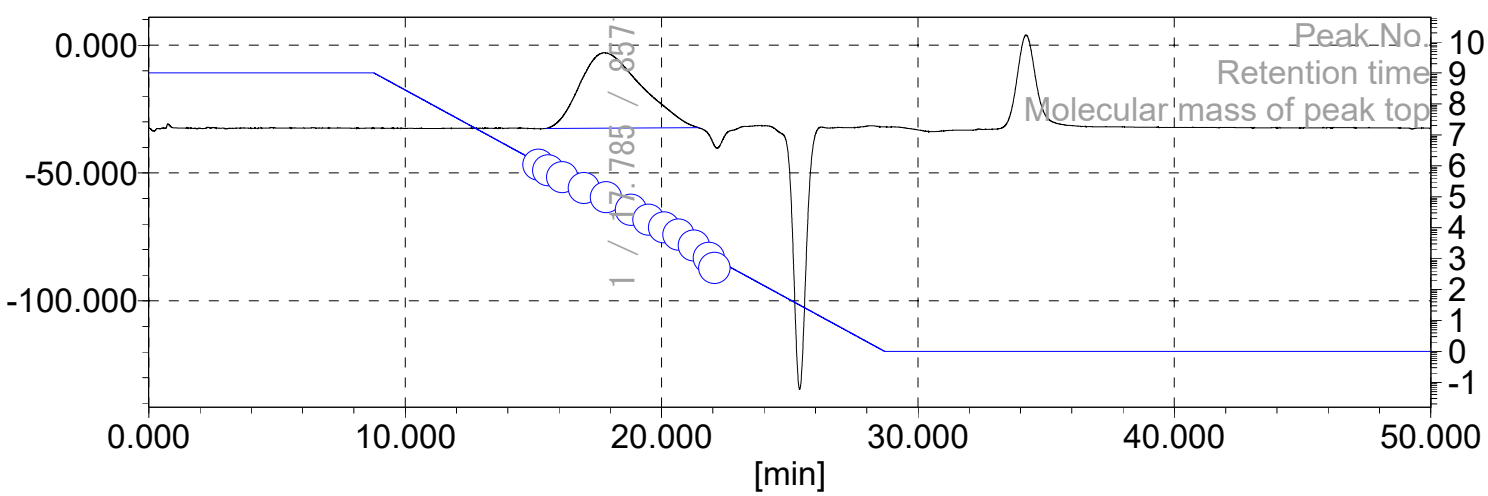

\begin{tabular}{|c|c|c|c|c|c|}
\hline & [min] & {$[\mathrm{mV}]$} & {$[\mathrm{mol}]$} & $\mathrm{Mn}$ & 28,187 \\
\hline Peak start & 15.488 & -32.571 & 935,719 & Mw & 98,478 \\
\hline Peak top & 17.785 & -2.992 & 85,712 & $\mathrm{Mz}$ & 212,466 \\
\hline \multirow[t]{2}{*}{ Peak end } & 21.445 & -32.312 & 1,899 & $\mathrm{Mz}+1$ & 328,444 \\
\hline & & & & Mv & 98,478 \\
\hline Height [mV] & & & 29.479 & $\mathrm{Mp}$ & 85,713 \\
\hline Area $\left[\mathrm{mV}^{*} \mathrm{~s}\right]$ & & & 4953.753 & $\mathrm{Mz} / \mathrm{Mw}$ & 2.157 \\
\hline Area\% [\%] & & & 100.000 & $\mathrm{Mw} / \mathrm{Mn}$ & 3.494 \\
\hline [eta] & & & 98478.43174 & $M z+1 / M w$ & 3.335 \\
\hline
\end{tabular}

Figure S108. HT-GPC data for optimization of 3 (entry 4).

$[\mathrm{mV}]$

[LogM]

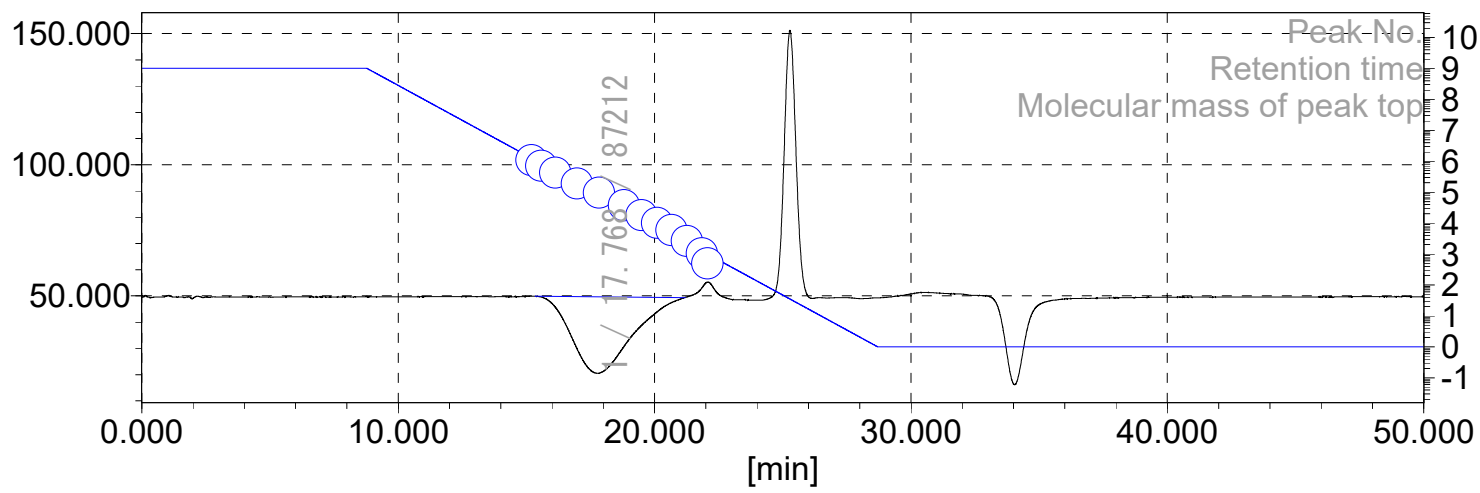

\begin{tabular}{|c|c|c|c|c|c|}
\hline & [min] & {$[\mathrm{mV}]$} & {$[\mathrm{mol}]$} & $\mathrm{Mn}$ & 35,167 \\
\hline Peak start & 15.335 & 49.880 & $1,097,621$ & Mw & 104,184 \\
\hline Peak top & 17.768 & 20.535 & 87,212 & $\mathrm{Mz}$ & 213,256 \\
\hline \multirow[t]{2}{*}{ Peak end } & 21.177 & 49.352 & 2,511 & $\mathrm{Mz}+1$ & 331,924 \\
\hline & & & & Mv & 104,184 \\
\hline Height $[\mathrm{mV}]$ & & & 29.125 & $\mathrm{Mp}$ & 86,760 \\
\hline Area $\left[\mathrm{mV}^{*} \mathrm{~s}\right]$ & & & 4481.107 & $\mathrm{Mz} / \mathrm{Mw}$ & 2.047 \\
\hline Area\% [\%] & & & 100.000 & $\mathrm{Mw} / \mathrm{Mn}$ & 2.963 \\
\hline [eta] & & & 104183.85838 & $\mathrm{Mz}+1 / \mathrm{Mw}$ & 3.186 \\
\hline
\end{tabular}

Figure S109. HT-GPC data for optimization of $\mathbf{3}$ (entry 6). 
$[\mathrm{mV}]$

[LogM]

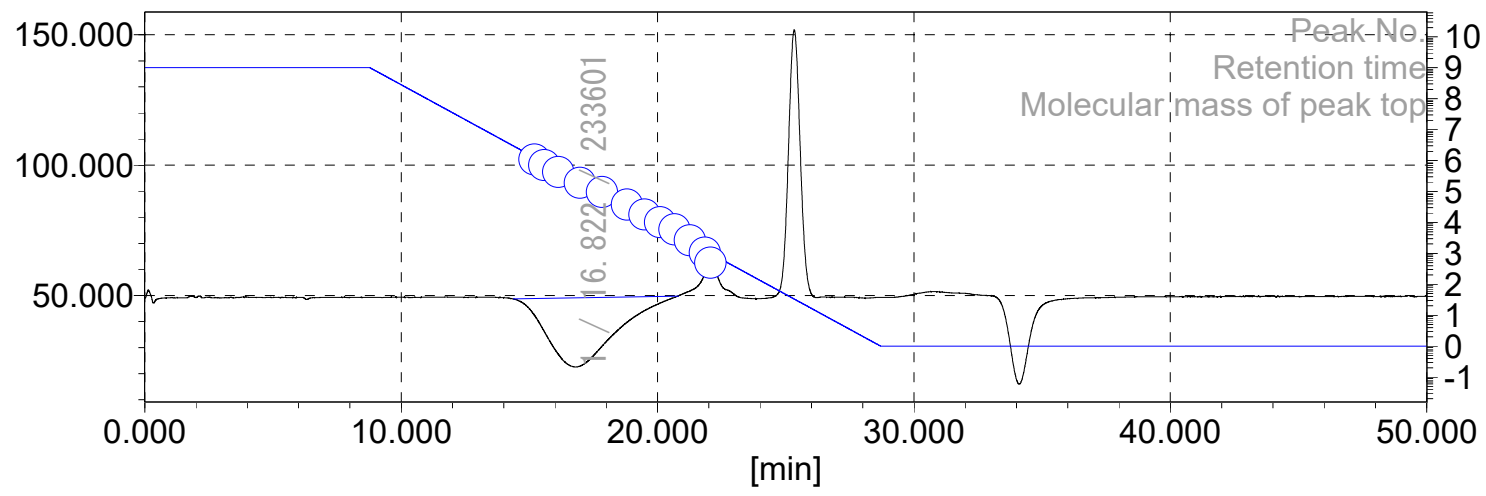

\begin{tabular}{lrrrlrr} 
& {$[\mathrm{min}]$} & {$[\mathrm{mV}]$} & \multicolumn{1}{c}{$[\mathrm{mol}]$} & & $\mathrm{Mn}$ & 68,976 \\
\hline \hline Peak start & 14.373 & 48.679 & $2,986,265$ & & $\mathrm{Mw}$ & 295,866 \\
Peak top & 16.822 & 22.586 & 233,601 & & $\mathrm{Mz}$ & 681,896 \\
Peak end & 20.792 & 49.778 & 3,749 & & $\mathrm{Mz}+1$ & $1,077,470$ \\
& & & & & $\mathrm{Mv}$ & 295,866 \\
Height [mV] & & & 26.512 & & $\mathrm{Mp}$ & 233,602 \\
Area [mV*s] & & & 4678.539 & & $\mathrm{Mz} / \mathrm{Mw}$ & 2.305 \\
Area\% [\%] & & & 100.000 & & $\mathrm{Mw} / \mathrm{Mn}$ & 4.289 \\
[eta] & & & 295865.63913 & & $\mathrm{Mz}+1 / \mathrm{Mw}$ & 3.642
\end{tabular}

Figure S110. HT-GPC data for optimization of 3 (entry 7).

[mV]

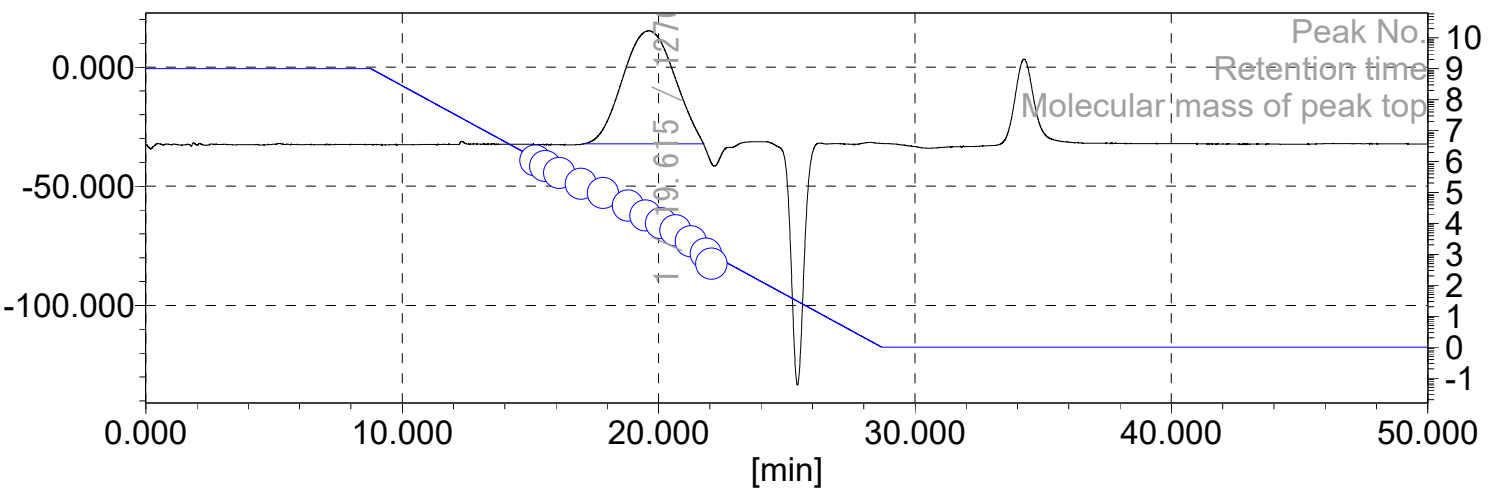

\begin{tabular}{|c|c|c|c|c|c|}
\hline & {$[\mathrm{min}]$} & {$[\mathrm{mV}]$} & {$[\mathrm{mol}]$} & $\mathrm{Mn}$ & 8,642 \\
\hline Peak start & 17.063 & -32.251 & 181,652 & Mw & 18,556 \\
\hline Peak top & 19.615 & 15.180 & 12,760 & $\mathrm{Mz}$ & 36,147 \\
\hline \multirow[t]{2}{*}{ Peak end } & 21.752 & -32.253 & 1,380 & $\mathrm{Mz}+1$ & 57,566 \\
\hline & & & & Mv & 18,556 \\
\hline Height [mV] & & & 47.432 & $\mathrm{Mp}$ & 12,673 \\
\hline Area $\left[\mathrm{mV}^{*} \mathrm{~s}\right]$ & & & 6642.487 & $\mathrm{Mz} / \mathrm{Mw}$ & 1.948 \\
\hline Area\% [\%] & & & 100.000 & $\mathrm{Mw} / \mathrm{Mn}$ & 2.147 \\
\hline [eta] & & & 18556.27114 & $M z+1 / M w$ & 3.102 \\
\hline
\end{tabular}

Figure S111. HT-GPC data for optimization of 3 (entry 8). 
$[\mathrm{mV}]$

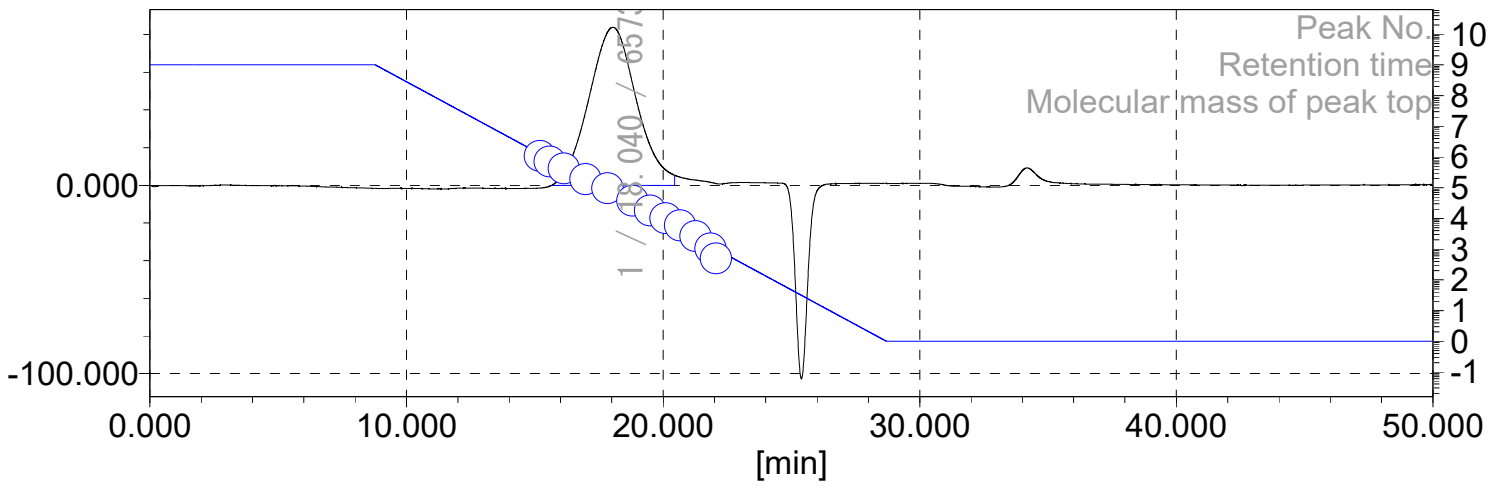

\begin{tabular}{|c|c|c|c|c|c|}
\hline & {$[\mathrm{min}]$} & {$[\mathrm{mV}]$} & {$[\mathrm{mol}]$} & $\mathrm{Mn}$ & 42,067 \\
\hline Peak start & 15.680 & 0.179 & 766,499 & Mw & 92,498 \\
\hline Peak top & 18.040 & 83.883 & 65,733 & $\mathrm{Mz}$ & 176,486 \\
\hline \multirow[t]{2}{*}{ Peak end } & 20.445 & 5.361 & 5,379 & $\mathrm{Mz}+1$ & 278,531 \\
\hline & & & & Mv & 92,498 \\
\hline Height [mV] & & & 83.910 & $\mathrm{Mp}$ & 65,733 \\
\hline Area $\left[\mathrm{mV}^{*} \mathrm{~s}\right]$ & & & 10780.058 & $\mathrm{Mz} / \mathrm{Mw}$ & 1.908 \\
\hline Area\% [\%] & & & 100.000 & $\mathrm{Mw} / \mathrm{Mn}$ & 2.199 \\
\hline [eta] & & & 92497.57350 & $\mathrm{Mz}+1 / \mathrm{Mw}$ & 3.011 \\
\hline
\end{tabular}

Figure S112. HT-GPC data of 3.

$[\mathrm{mV}]$

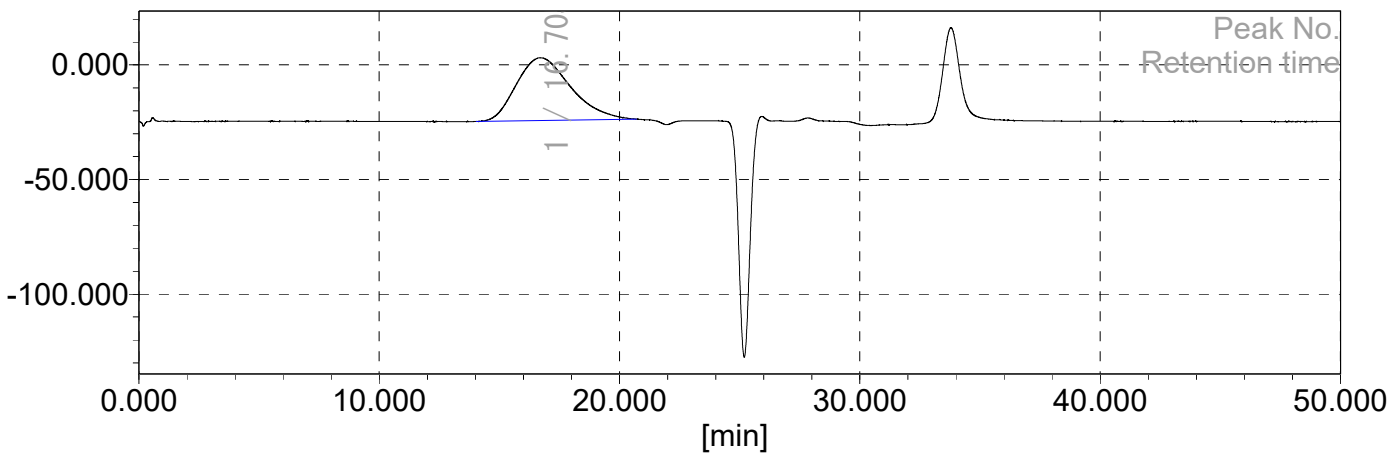

\begin{tabular}{lrrrlrr} 
& {$[\mathrm{min}]$} & \multicolumn{1}{c}{$[\mathrm{mV}]$} & \multicolumn{1}{c}{$[\mathrm{mol}]$} & & $\mathrm{Mn}$ & 101,903 \\
\hline Peak start & 14.028 & -24.655 & $4,276,310$ & & $\mathrm{Mw}$ & 336,931 \\
Peak top & 16.705 & 3.141 & 263,760 & & $\mathrm{Mz}$ & 711,511 \\
Peak end & 20.677 & -23.646 & 4,226 & & $\mathrm{Mz}+1$ & $1,136,509$ \\
& & & & & $\mathrm{Mv}$ & 336,931 \\
Height [mV] & & & 27.390 & & $\mathrm{Mp}$ & 263,760 \\
Area [mV*s] & & & 4279.505 & & $\mathrm{Mz} / \mathrm{Mw}$ & 2.112 \\
Area\% [\%] & & & 100.000 & & $\mathrm{Mw} / \mathrm{Mn}$ & 3.306 \\
[eta] & & & 336931.47306 & & $\mathrm{Mz}+1 / \mathrm{Mw}$ & 3.373
\end{tabular}

Figure S113. HT-GPC data of 6. 
$[\mathrm{mV}]$

[LogM]

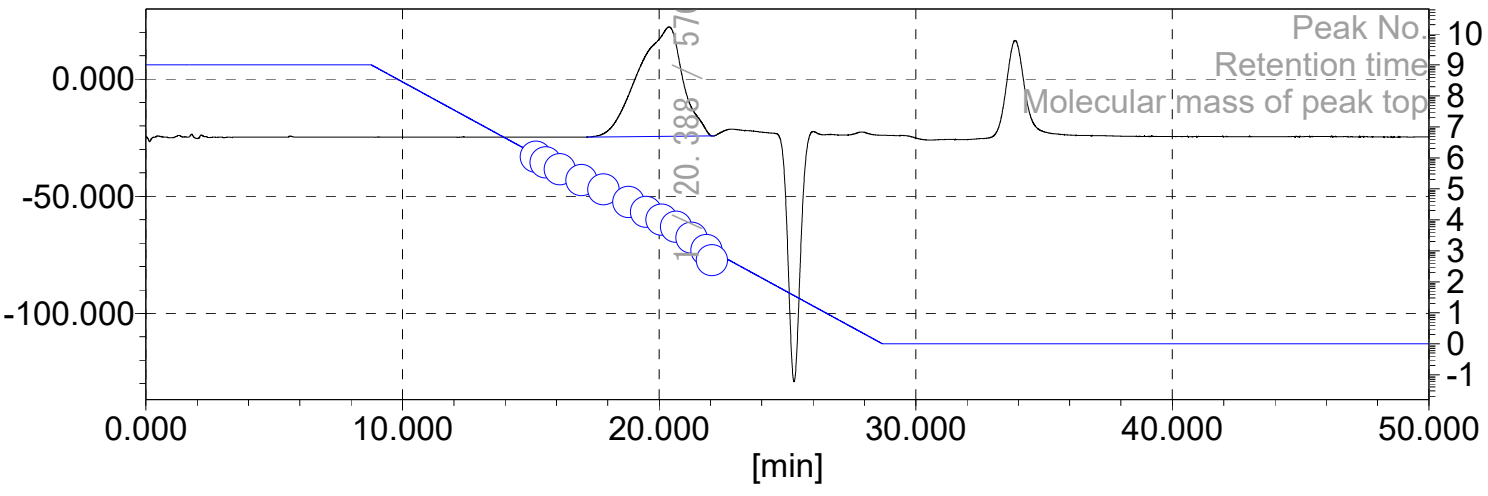

\begin{tabular}{|c|c|c|c|c|c|}
\hline & {$[\mathrm{min}]$} & {$[\mathrm{mV}]$} & {$[\mathrm{mol}]$} & $\mathrm{Mn}$ & 6,352 \\
\hline Peak start & 17.178 & -24.695 & 161,161 & Mw & 12,490 \\
\hline Peak top & 20.388 & 22.266 & 5,706 & $\mathrm{Mz}$ & 24,366 \\
\hline \multirow[t]{2}{*}{ Peak end } & 22.137 & -24.281 & 924 & $M z+1$ & 40,460 \\
\hline & & & & Mv & 12,490 \\
\hline Height [mV] & & & 46.693 & $\mathrm{Mp}$ & 5,706 \\
\hline Area $\left[\mathrm{mV}^{*} \mathrm{~s}\right]$ & & & 5611.380 & $\mathrm{Mz} / \mathrm{Mw}$ & 1.951 \\
\hline Area\% [\%] & & & 100.000 & $\mathrm{Mw} / \mathrm{Mn}$ & 1.966 \\
\hline [eta] & & & 12490.00561 & $M z+1 / M w$ & 3.239 \\
\hline
\end{tabular}

\section{Figure S114. HT-GPC data of 7.}

[mV]

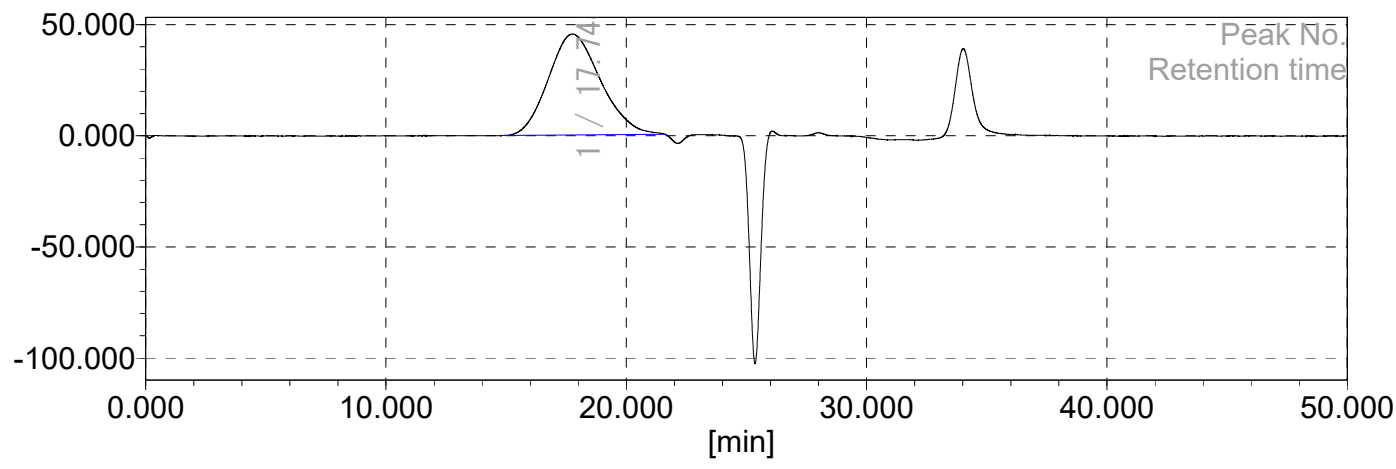

\begin{tabular}{lcrrlrr} 
& {$[\mathrm{min}]$} & \multicolumn{1}{c}{$[\mathrm{mV}]$} & \multicolumn{1}{c}{$[\mathrm{mol}]$} & & $\mathrm{Mn}$ & 39,430 \\
\hline \hline Peak start & 14.797 & 0.067 & $1,922,120$ & & $\mathrm{Mw}$ & 122,885 \\
Peak top & 17.748 & 45.795 & 89,047 & & $\mathrm{Mz}$ & 277,144 \\
Peak end & 21.598 & 0.703 & 1,619 & & $\mathrm{Mz}+1$ & 482,771 \\
& & & & $\mathrm{Mv}$ & 122,885 \\
Height [mV] & & & 45.452 & & $\mathrm{Mp}$ & 89,047 \\
Area [mV*s] & & & 6871.223 & & $\mathrm{Mz} / \mathrm{Mw}$ & 2.255 \\
Area\% [\%] & & 100.000 & & $\mathrm{Mw} / \mathrm{Mn}$ & 3.117 \\
[eta] & & 122884.55508 & & $\mathrm{Mz}+1 / \mathrm{Mw}$ & 3.929
\end{tabular}

Figure S115. HT-GPC data of 8. 
$\mathrm{RI}[\mathrm{mV}]$

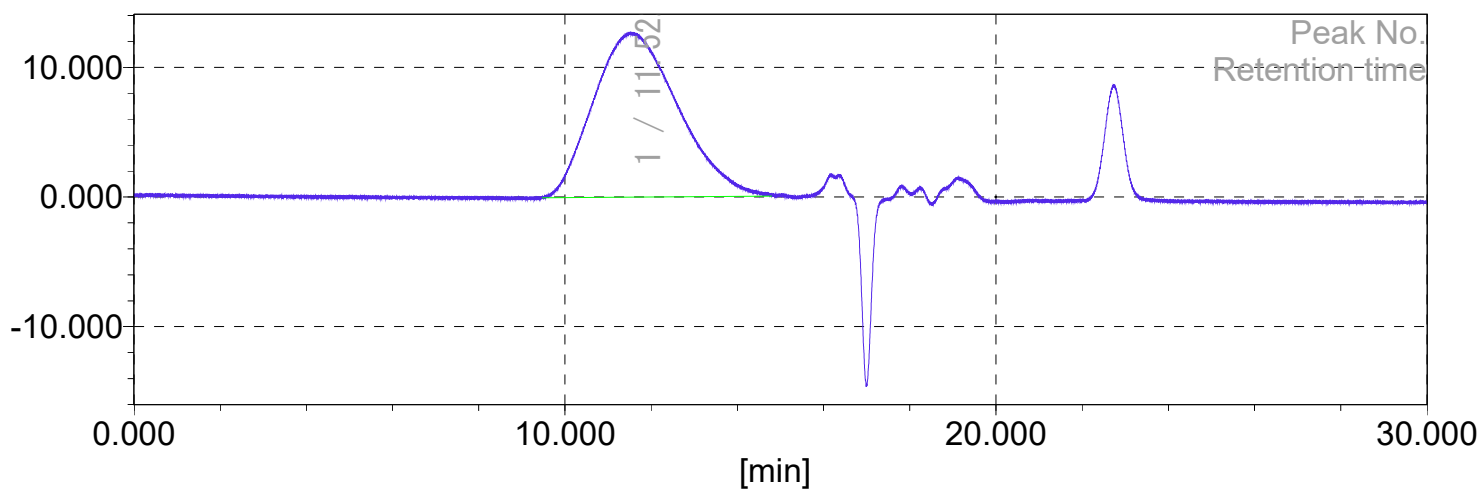

\begin{tabular}{|c|c|c|c|c|c|}
\hline & {$[\mathrm{min}]$} & {$[\mathrm{mV}]$} & {$[\mathrm{mol}]$} & Mn & 60,438 \\
\hline Peak start & 9.475 & -0.063 & $1,024,214$ & Mw & 143,312 \\
\hline Peak top & 11.522 & 12.712 & 121,984 & $\mathrm{Mz}$ & 257,014 \\
\hline \multirow[t]{2}{*}{ Peak end } & 14.780 & 0.067 & 4,122 & $\mathrm{Mz}+1$ & 372,197 \\
\hline & & & & Mv & 143,312 \\
\hline Height [mV] & & & 12.725 & $\mathrm{Mp}$ & 121,985 \\
\hline Area $\left[\mathrm{mV}^{*} \mathrm{sec}\right]$ & & & 1730.247 & $\mathrm{Mz} / \mathrm{Mw}$ & 1.793 \\
\hline Height\% [\%] & & & 100.000 & $\mathrm{Mw} / \mathrm{Mn}$ & 2.371 \\
\hline [eta] & & & 143311.58762 & $\mathrm{Mz}+1 / \mathrm{Mw}$ & 2.597 \\
\hline
\end{tabular}

Figure S116. HT-GPC data of 9.

$[\mathrm{mV}]$

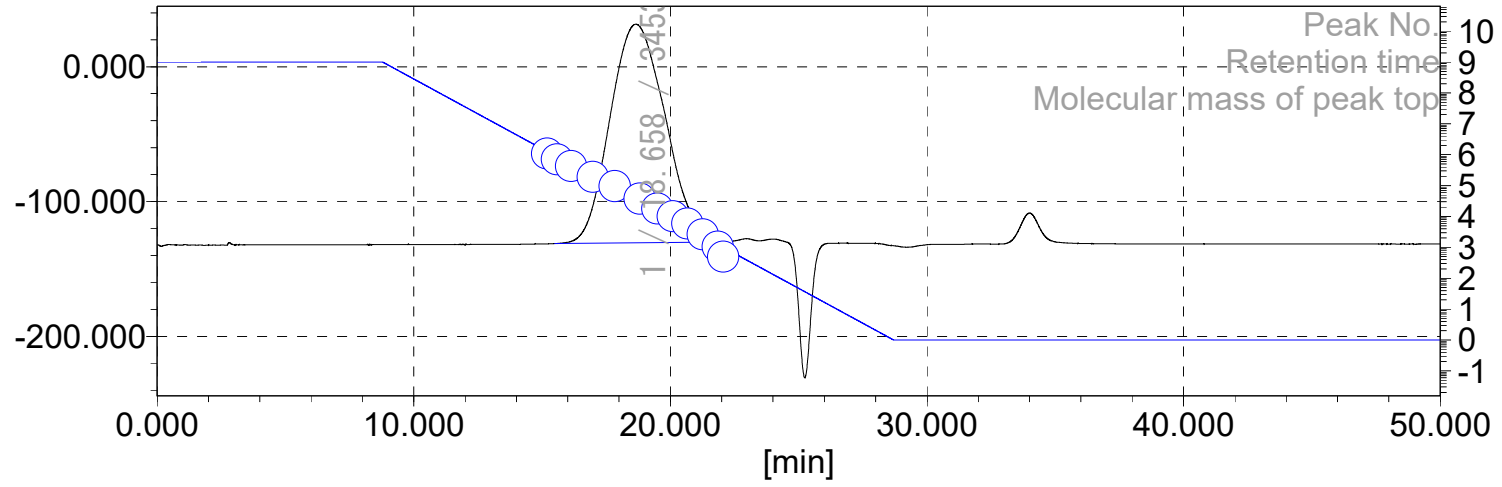

\begin{tabular}{lrrrlrr} 
& {$[\mathrm{min}]$} & \multicolumn{1}{c}{$[\mathrm{mV}]$} & \multicolumn{1}{c}{$[\mathrm{mol}]$} & & $\mathrm{Mn}$ & 18,203 \\
\hline \hline Peak start & 15.488 & -131.196 & 935,719 & & $\mathrm{Mw}$ & 48,122 \\
Peak top & 18.658 & 31.599 & 34,538 & & $\mathrm{Mz}$ & 104,763 \\
Peak end & 21.752 & -130.023 & 1,380 & & $\mathrm{Mz}+1$ & 188,556 \\
& & & & & $\mathrm{Mv}$ & 48,122 \\
Height [mV] & & & 162.201 & & $\mathrm{Mp}$ & 34,538 \\
Area [mV*s] & & & 24169.166 & & $\mathrm{Mz} / \mathrm{Mw}$ & 2.177 \\
Area\% [\%] & & 100.000 & & $\mathrm{Mw} / \mathrm{Mn}$ & 2.644 \\
[eta] & & 48121.88044 & & $\mathrm{Mz}+1 / \mathrm{Mw}$ & 3.918
\end{tabular}

Figure S117. HT-GPC data of 10. 
$[\mathrm{mV}]$

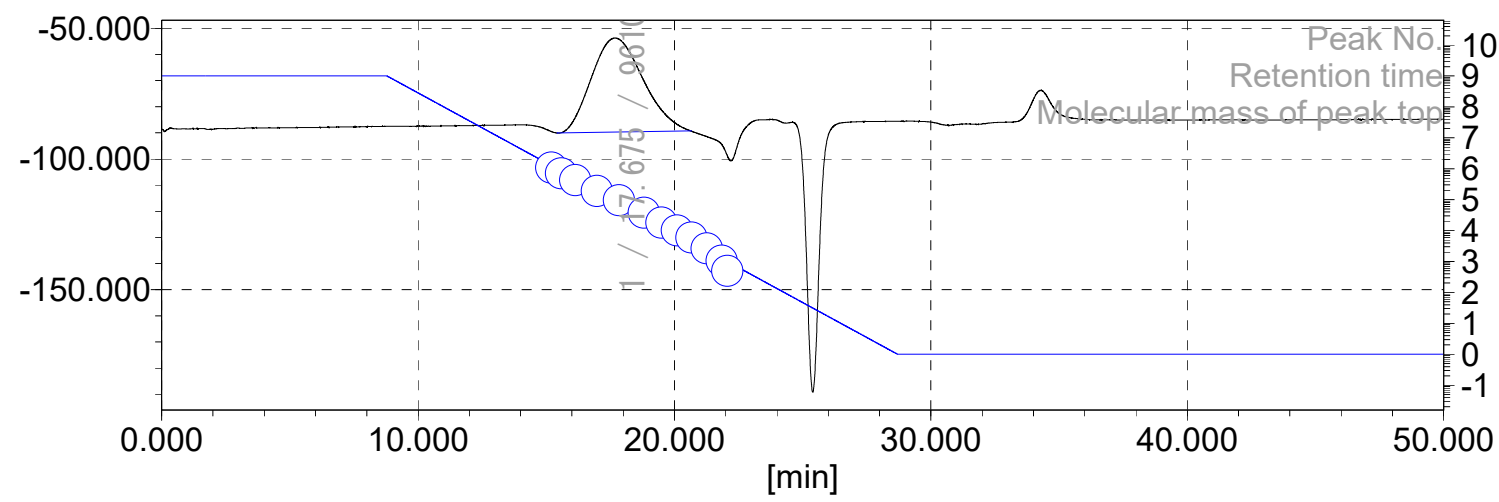

[LogM]

\begin{tabular}{lcrrllr} 
& {$[\mathrm{min}]$} & {$[\mathrm{mV}]$} & \multicolumn{1}{c}{$[\mathrm{mol}]$} & & $\mathrm{Mn}$ & 50,401 \\
\hline \hline Peak start & 15.412 & -90.098 & $1,013,442$ & & $\mathrm{Mw}$ & 118,609 \\
Peak top & 17.675 & -53.757 & 96,109 & & $\mathrm{Mz}$ & 218,596 \\
Peak end & 20.638 & -89.314 & 4,398 & & $\mathrm{Mz}+1$ & 322,260 \\
& & & & & $\mathrm{Mv}$ & 118,609 \\
Height [mV] & & & 36.002 & & $\mathrm{Mp}$ & 96,110 \\
Area [mV*s] & & & 5036.410 & & $\mathrm{Mz} / \mathrm{Mw}$ & 1.843 \\
Area\% [\%] & & & 100.000 & & $\mathrm{Mw} / \mathrm{Mn}$ & 2.353 \\
[eta] & & & 118609.38304 & & $\mathrm{Mz}+1 / \mathrm{Mw}$ & 2.717
\end{tabular}

Figure S118. HT-GPC data of 11.

[mV]

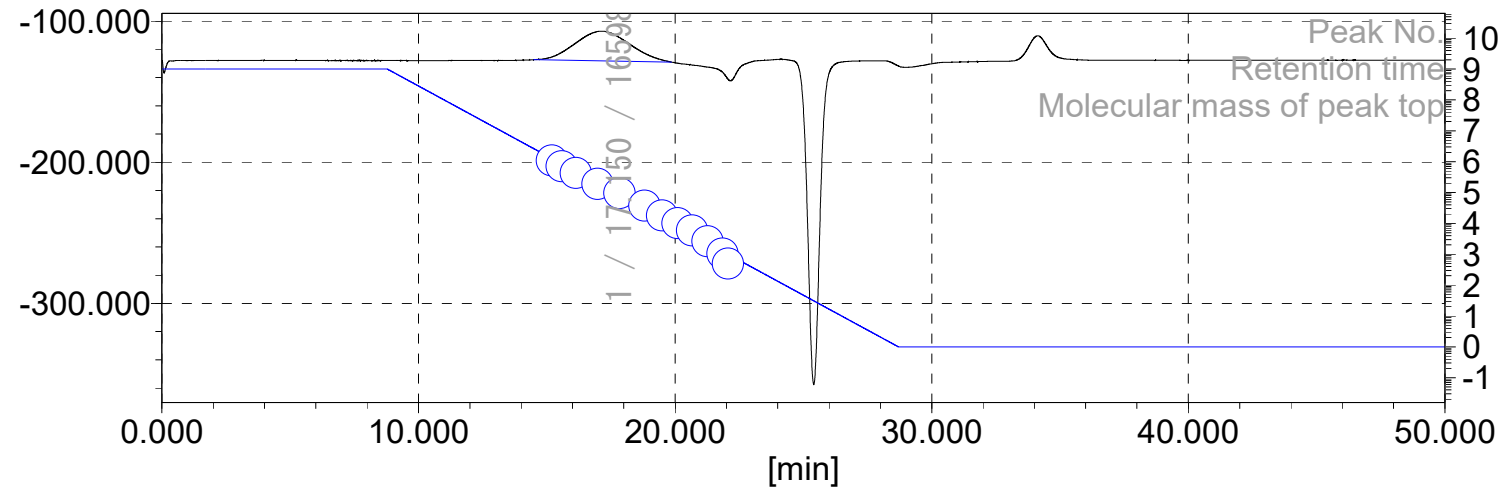

\begin{tabular}{lrrrllr} 
& {$[\mathrm{min}]$} & {$[\mathrm{mV}]$} & \multicolumn{1}{c}{$[\mathrm{mol}]$} & & $\mathrm{Mn}$ & 97,912 \\
\hline \hline Peak start & 14.488 & -127.415 & $2,649,403$ & & $\mathrm{Mw}$ & 246,408 \\
Peak top & 17.150 & -107.206 & 165,984 & & $\mathrm{Mz}$ & 523,360 \\
Peak end & 19.832 & -128.987 & 10,184 & & $\mathrm{Mz}+1$ & 865,928 \\
& & & & & $\mathrm{Mv}$ & 246,408 \\
Height [mV] & & & 20.992 & & $\mathrm{Mp}$ & 165,985 \\
Area [mV*s] & & & 3098.102 & & $\mathrm{Mz} / \mathrm{Mw}$ & 2.124 \\
Area\% [\%] & & & 100.000 & & $\mathrm{Mw} / \mathrm{Mn}$ & 2.517 \\
[eta] & & & 246408.16930 & & $\mathrm{Mz}+1 / \mathrm{Mw}$ & 3.514
\end{tabular}

Figure S119. HT-GPC data of 12. 
$[\mathrm{mV}]$

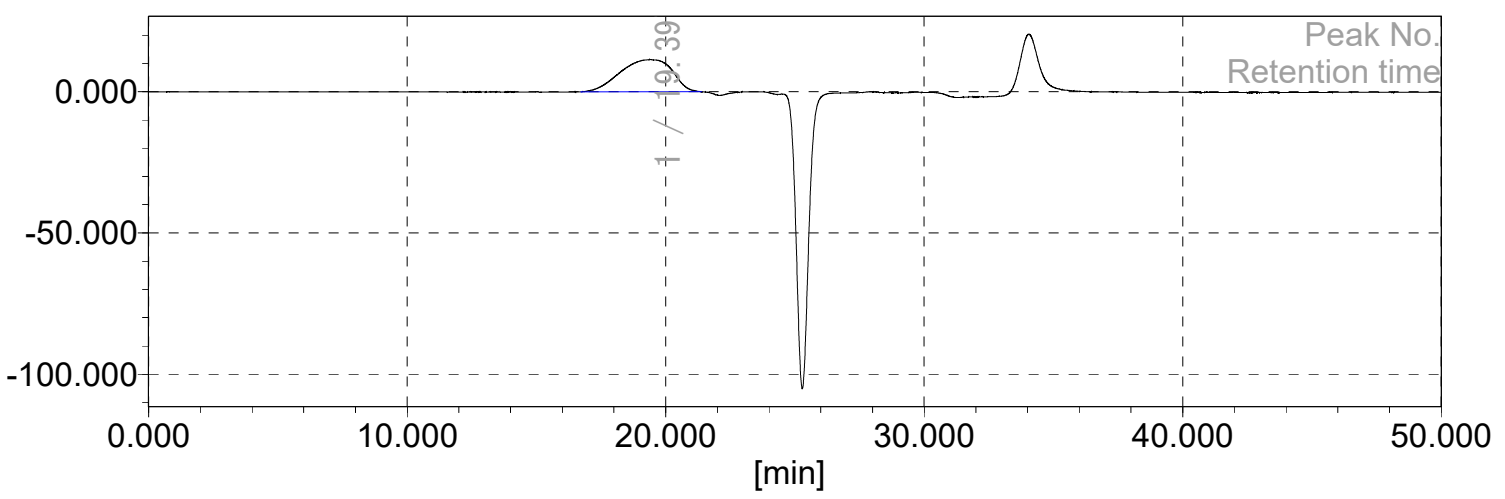

\begin{tabular}{lcrrllr} 
& {$[\mathrm{min}]$} & {$[\mathrm{mV}]$} & \multicolumn{1}{c}{$[\mathrm{mol}]$} & & $\mathrm{Mn}$ & 14,103 \\
\hline \hline Peak start & 16.718 & -0.055 & 260,125 & & $\mathrm{Mw}$ & 28,468 \\
Peak top & 19.397 & 11.360 & 16,016 & & $\mathrm{Mz}$ & 55,281 \\
Peak end & 21.368 & 0.024 & 2,057 & $\mathrm{Mz}+1$ & 87,673 \\
& & & & $\mathrm{Mv}$ & 28,468 \\
Height [mV] & & & 11.369 & $\mathrm{Mp}$ & 16,017 \\
Area [mV*s] & & & 1586.423 & $\mathrm{Mz} / \mathrm{Mw}$ & 1.942 \\
Area\% [\%] & & & 100.000 & $\mathrm{Mw} / \mathrm{Mn}$ & 2.019 \\
[eta] & & & 28468.39072 & & $\mathrm{Mz}+1 / \mathrm{Mw}$ & 3.080
\end{tabular}

\section{Figure S120. HT-GPC data of 13.}

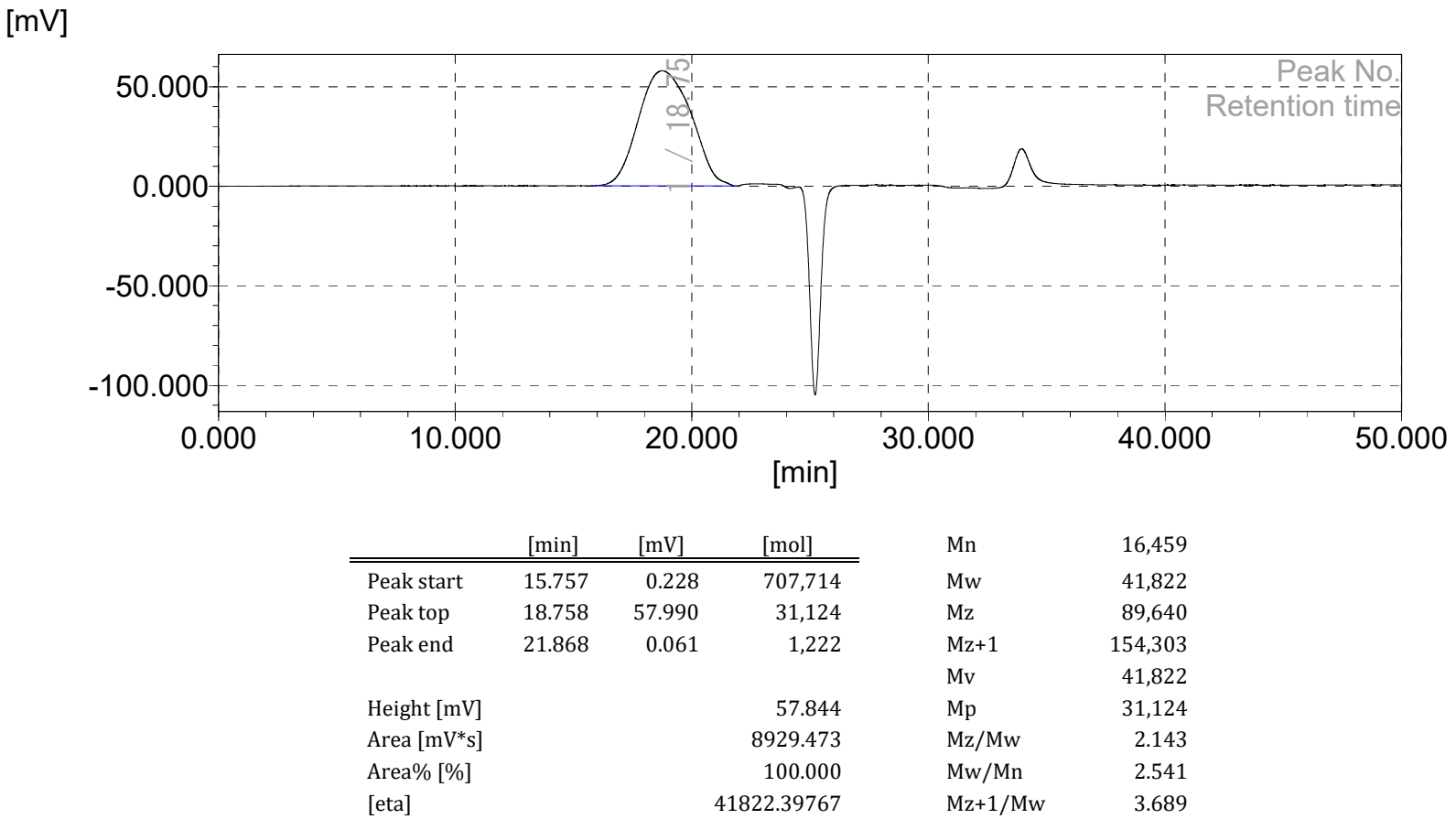

Figure S121. HT-GPC data of 14. 
[mV]

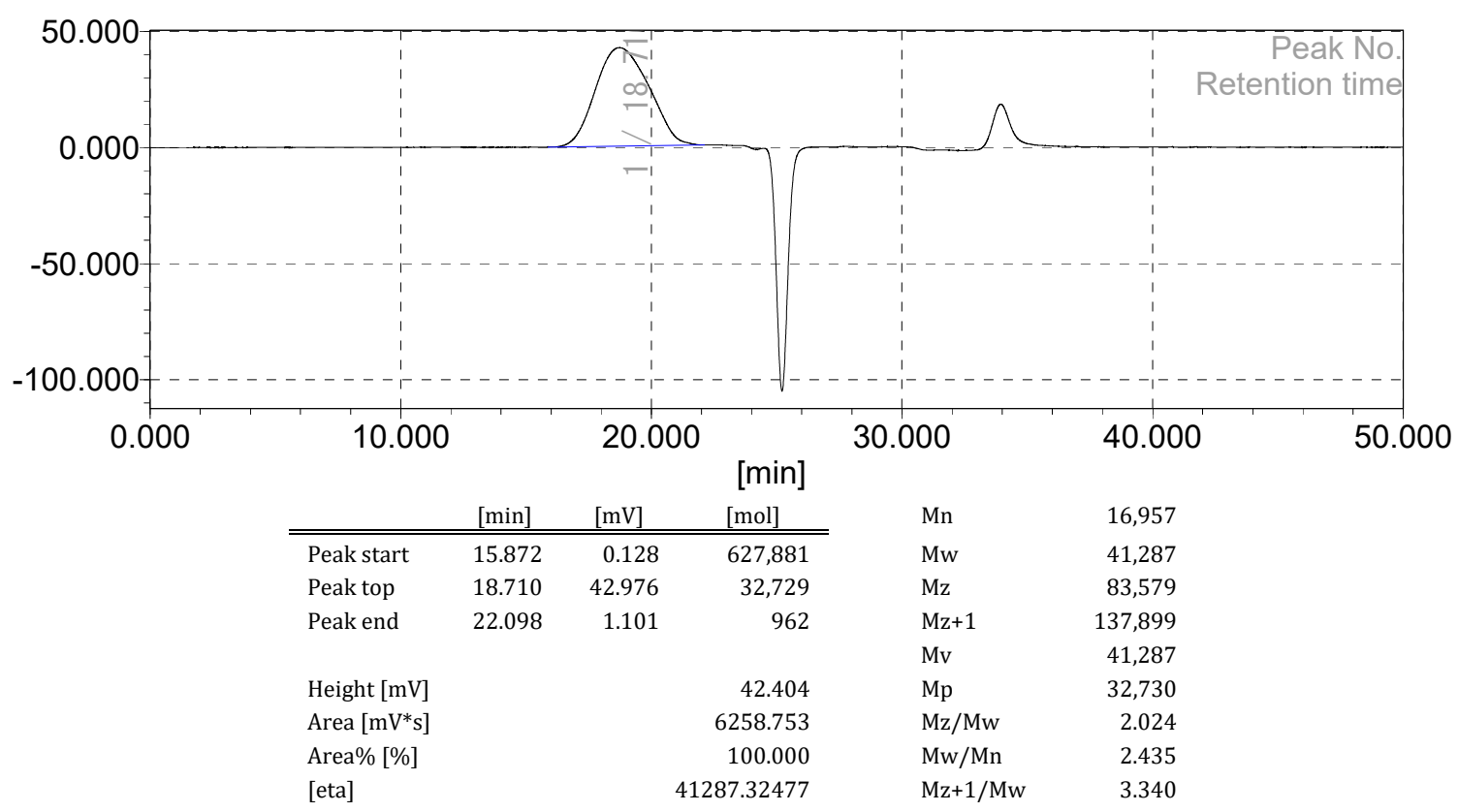

Figure S122. HT-GPC data of 15.

[mV]

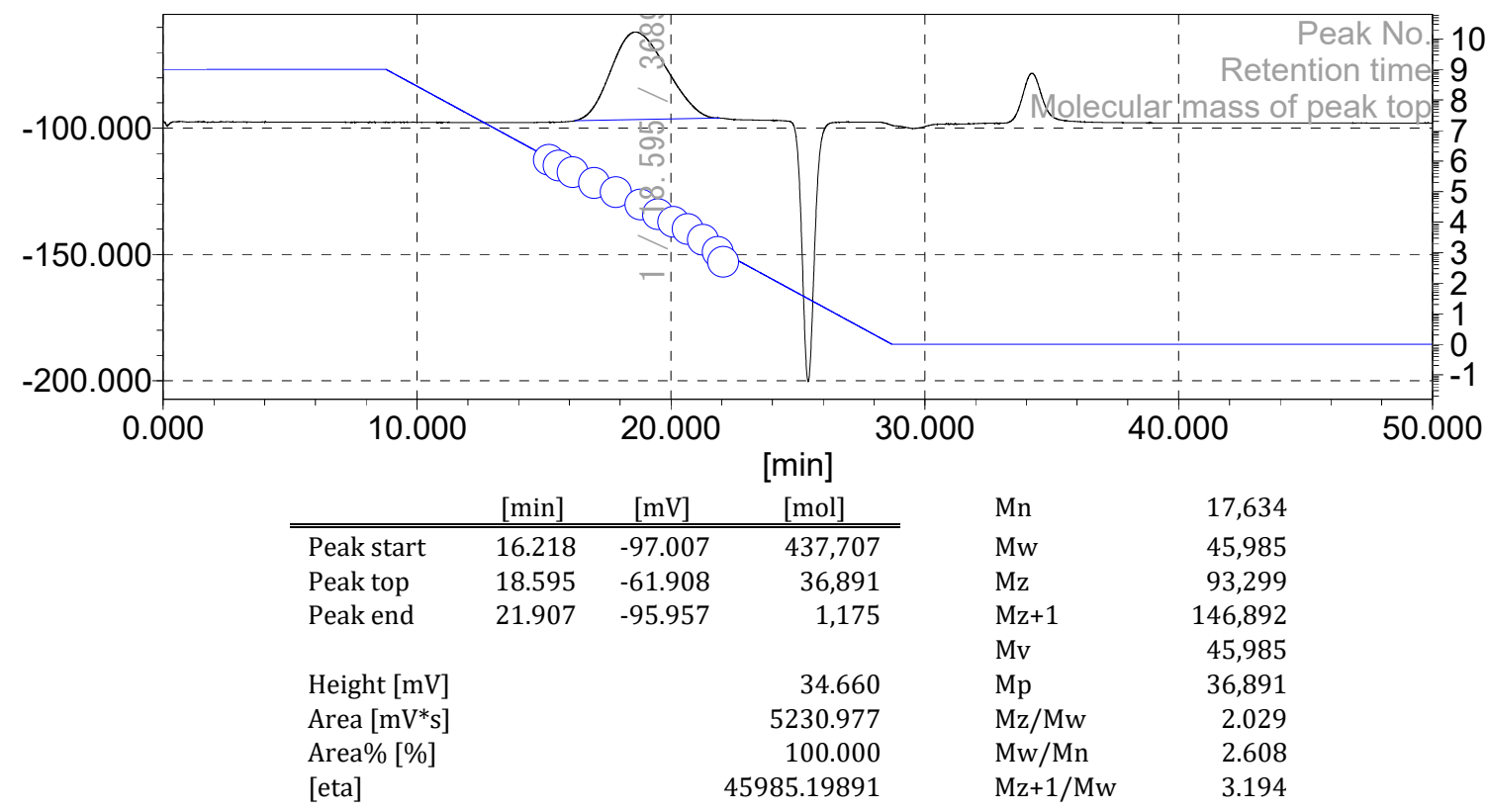

Figure S123. HT-GPC data of 16. 


\section{X-Ray Diffraction Data}

\section{AzoCarbazole $\mathrm{B}\left(\mathrm{C}_{6} \mathrm{~F}_{5}\right)_{3} / \mathrm{H}_{2} \mathrm{O}$ adduct (17a)}

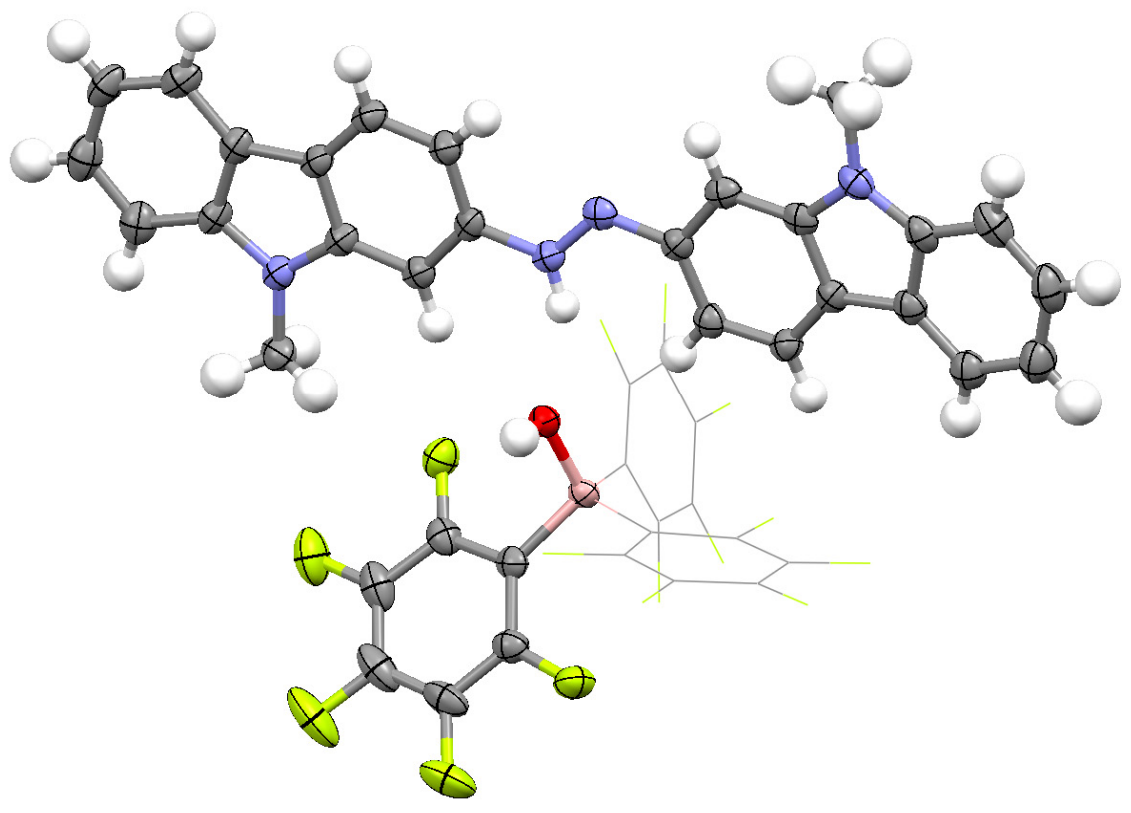

\begin{tabular}{|c|c|}
\hline \multicolumn{2}{|l|}{ Crystal data } \\
\hline Chemical formula & $2\left(\mathrm{C}_{18} \mathrm{HBF}_{15} \mathrm{O}\right) \cdot \mathrm{C}_{26} \mathrm{H}_{20} \mathrm{~N}_{4} \cdot 2\left(\mathrm{C}_{26} \mathrm{H}_{21} \mathrm{~N}_{4}\right)$ \\
\hline$M_{\mathrm{r}}$ & 2225.39 \\
\hline Crystal system, space group & Triclinic, $P-1$ \\
\hline Temperature (K) & 150 \\
\hline$a, b, c(\AA)$ & $15.0605(16), 17.2367(17), 20.238(2)$ \\
\hline$\alpha, \beta, \gamma\left({ }^{\circ}\right)$ & $67.095(6), 88.512(7), 88.113(7)$ \\
\hline$V\left(\AA^{3}\right)$ & $4836.4(9)$ \\
\hline$Z$ & 2 \\
\hline Radiation type & $\mathrm{Cu} K \alpha$ \\
\hline$\mu\left(\mathrm{mm}^{-1}\right)$ & 1.16 \\
\hline Crystal size $(\mathrm{mm})$ & $0.19 \times 0.14 \times 0.05$ \\
\hline \multicolumn{2}{|l|}{ Data collection } \\
\hline Diffractometer & $\begin{array}{l}\text { Bruker AXS D8 Quest diffractometer with PhotonIII_C14 } \\
\text { charge-integrating and photon counting pixel array detector }\end{array}$ \\
\hline Absorption correction & $\begin{array}{l}\text { Multi-scan, TWINABS 2012/1: Krause, L., Herbst-Irmer, R., } \\
\text { Sheldrick G.M. \& Stalke D. (2015). J. Appl. Cryst. } 48 \text { 3-10. }\end{array}$ \\
\hline$T_{\min }, T_{\max }$ & $0.589,0.754$ \\
\hline $\begin{array}{l}\text { No. of measured, independent and observed }[I>2 \sigma(I)] \\
\text { reflections }\end{array}$ & $70344,19498,16118$ \\
\hline$R_{\text {int }}$ & 0.065 \\
\hline
\end{tabular}




\begin{tabular}{|l|l|}
\hline$(\sin \theta / \lambda)_{\max }\left(\AA^{-1}\right)$ & 0.641 \\
\hline \multicolumn{2}{|l|}{} \\
\hline Refinement & $0.063,0.173,1.05$ \\
\hline$R\left[F^{2}>2 \sigma\left(F^{2}\right)\right], w R\left(F^{2}\right), S$ & 19498 \\
\hline No. of reflections & 1451 \\
\hline No. of parameters & H-atom parameters constrained \\
\hline H-atom treatment & $0.49,-0.31$ \\
\hline$\Delta \rho_{\max }, \Delta \rho_{\min }\left(\mathrm{e} \AA^{-3}\right)$ & \\
\hline
\end{tabular}

Structure Solution and Refinement. The crystals under investigation were multi-component with multiple domains. Most crystals screened did not diffract to sufficient resolution. The crystal chosen for data collection featured one dominant major domain with sufficient medium and high angle data for collection of acceptable resolution data, and several smaller domains. Transformation matrices identified using the program Cell_Now were ambiguous, suggesting that minor domains were not related to the major domain by a typical twin relationship. Analysis of data using only the major domain did however indicate presence of twinning, with apparent difference densities of one to two electrons pert cubic Angstrom in positions incompatible with disorder, ill-defined ADPs, and higher than expected R values. Application of the Cell_Now suggested transformation matrices and integration of the data as two component did not result in reduction of these effects. The possibility of twinning was instead investigated using the program Rotax (Cooper et al., 2001) as embedded in WinGX (Farrugia, 2012), which identified the most likely type of twinning present to be a 180 degree rotation around the reciprocal $\left[\begin{array}{lll}1 & 0 & 0\end{array}\right]$ reciprocal lattice direction, with a twin transformation matrix of $1-0.047-0.023,0-10,00-1$. Application of the approximate matrix $100,0-10,00-1$ using a TWIN command in Shelx resulted in some reduction of effects from twinning. The exact twinning matrix as obtained from Rotax was applied to the major component and the data were subsequently integrated using SAINT using both components and corrected for absorption using twinabs, resulting in the following statistics:

41682 data (13063 unique) involve domain 1 only, mean I/sigma 11.1

41549 data (13057 unique) involve domain 2 only, mean I/sigma 7.9

28815 data (11803 unique) involve 2 domains, mean I/sigma 18.2

23 data (23 unique) involve 3 domains, mean I/sigma 23.2

The exact twin matrix identified by the integration program was found to be:

$$
\begin{array}{lll}
1.00082 & -0.03854 & -0.01864 \\
0.02951 & -0.99920 & -0.00326 \\
0.02744 & 0.00358 & -1.00161
\end{array}
$$

The Rint value given is for all reflections and is based on agreement between observed single and composite intensities and those calculated from refined unique intensities and twin fractions (TWINABS (Sheldrick, 2012)). 


\section{DFT Calculations and Optimized Structures}

Computational Methods. Geometry optimizations were performed using the Gaussian 16 software package.$^{20}$ All geometries were fully optimized at the B3LYP/6-31G(d,p) level of DFT. A polarizable continuum model (PCM) was used to apply solvent corrections for all compounds in $\mathrm{C}_{6} \mathrm{H}_{5} \mathrm{Cl}$.
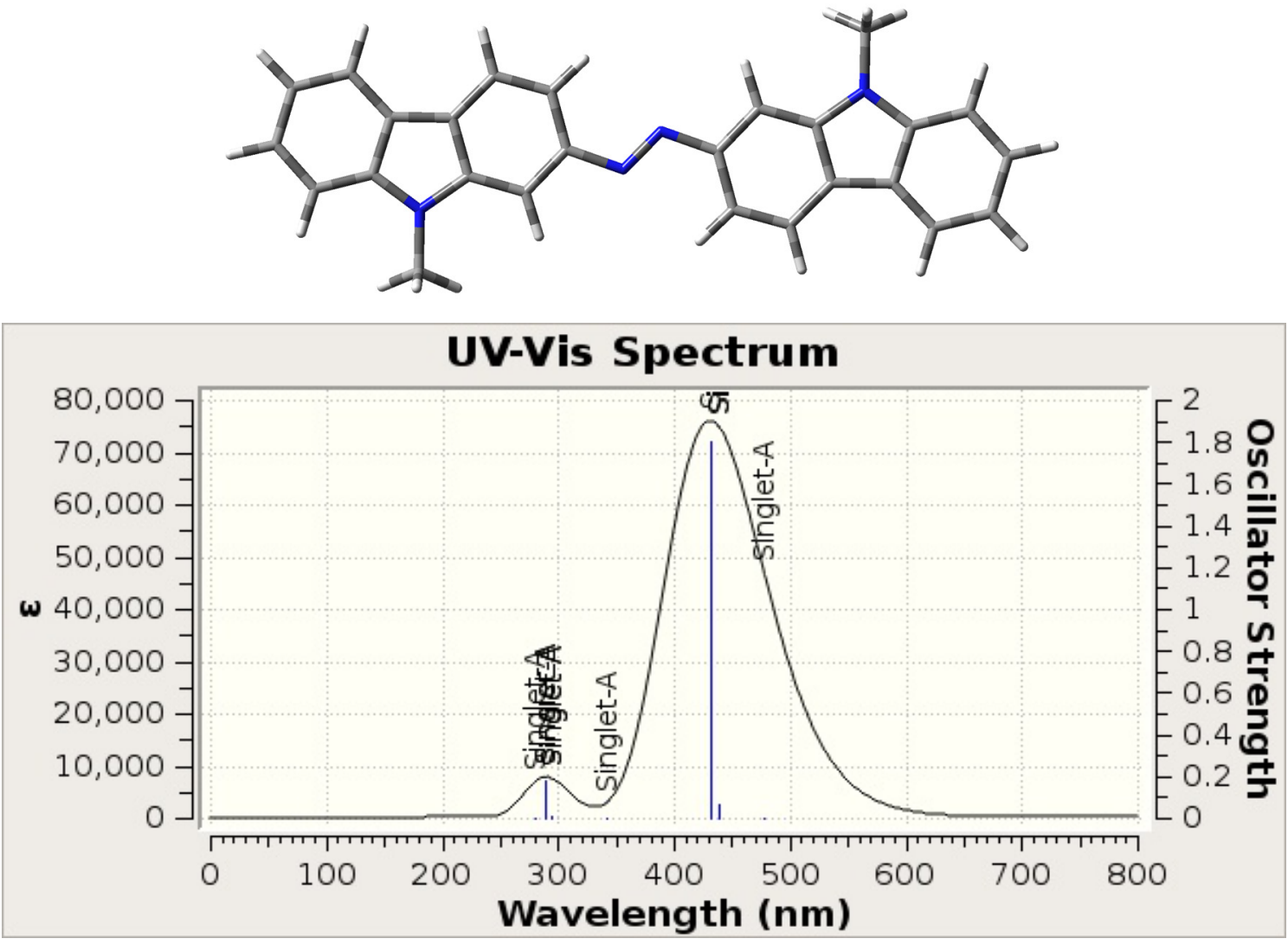

\begin{tabular}{|c|c|c|c|}
\hline TD State & Wavelength (nm) & Oscillator Strength & $\varepsilon$ \\
\hline 1 & 479.66 & 0.00080 & 2673 \\
\hline 2 & 437.44 & 0.076 & 253888 \\
\hline 3 & 436.62 & 0 & 0 \\
\hline 4 & 433.17 & 1.8321 & 0120375 \\
\hline 5 & 341.12 & 0 & 0 \\
\hline 6 & 294.32 & 0 & 30066 \\
\hline 7 & 292.98 & 0.0090 & 599978 \\
\hline 8 & 289.12 & 0.1796 & 0 \\
\hline 9 & 288.60 & 0 & \\
\hline
\end{tabular}




\begin{tabular}{|l|l|l|l|}
\hline 10 & 279.95 & 0 & 0 \\
\hline
\end{tabular}

Functional and Basis Set: B3LYP/6-31G(d,p)

Charge: 0

Multiplicity: 1

Imaginary Frequencies: 0

Sum of electronic and thermal Free Energies: -1221.527517 Hartrees

\section{Cartesian Coordinates:}

$\begin{array}{llll}\mathrm{C} & 5.91622800 & -0.78649700 & 0.01062200 \\ \mathrm{C} & 4.47352500 & -0.76159500 & 0.00173500 \\ \mathrm{C} & 4.09130800 & 0.60849000 & -0.02312300 \\ \mathrm{C} & 2.74985200 & 0.98806200 & -0.02282100 \\ \mathrm{C} & 3.48023500 & -1.75533800 & 0.01352600 \\ \mathrm{C} & 1.77721500 & -0.02054700 & -0.01059900 \\ \mathrm{C} & 2.14470600 & -1.38921000 & 0.00672400 \\ \mathrm{H} & 3.75872400 & -2.80519100 & 0.03054100 \\ \mathrm{H} & 1.35902400 & -2.13435200 & 0.01766800 \\ \mathrm{H} & 2.42845900 & 2.02348000 & -0.02735800 \\ \mathrm{~N} & 0.44428900 & 0.45049200 & -0.01176500 \\ \mathrm{C} & -2.14470600 & 1.38921000 & 0.00671300 \\ \mathrm{C} & -3.48023500 & 1.75533800 & 0.01351500 \\ \mathrm{C} & -1.77721500 & 0.02054700 & -0.01059400 \\ \mathrm{C} & -4.47352500 & 0.76159500 & 0.00173900 \\ \mathrm{H} & -3.75872400 & 2.80519100 & 0.03051900 \\ \mathrm{C} & -2.74985300 & -0.98806300 & -0.02280200 \\ \mathrm{C} & -4.09130800 & -0.60849000 & -0.02310500 \\ \mathrm{H} & -2.42845900 & -2.02348000 & -0.02732800 \\ \mathrm{C} & -6.34436600 & -0.57089800 & -0.00966200\end{array}$




\begin{tabular}{|c|c|c|c|}
\hline $\mathrm{C}$ & -5.91622800 & 0.78649700 & 0.01063000 \\
\hline $\mathrm{C}$ & -7.70250000 & -0.90892000 & 0.00618300 \\
\hline $\mathrm{C}$ & -6.87055300 & 1.81299600 & 0.03502700 \\
\hline $\mathrm{C}$ & -8.62789000 & 0.13174800 & 0.03056600 \\
\hline $\mathrm{H}$ & -8.03081400 & -1.94292800 & 0.00519900 \\
\hline $\mathrm{C}$ & -8.22114700 & 1.47958500 & 0.04397100 \\
\hline $\mathrm{H}$ & -6.55829500 & 2.85333400 & 0.04941400 \\
\hline $\mathrm{H}$ & -9.68780300 & -0.10453900 & 0.04315300 \\
\hline $\mathrm{H}$ & -8.97126700 & 2.26398400 & 0.06430700 \\
\hline $\mathrm{C}$ & 6.34436600 & 0.57089800 & -0.00968700 \\
\hline C & 7.70250000 & 0.90892000 & 0.00615100 \\
\hline C & 6.87055300 & -1.81299600 & 0.03502800 \\
\hline $\mathrm{C}$ & 8.62789000 & -0.13174700 & 0.03054300 \\
\hline $\mathrm{H}$ & 8.03081400 & 1.94292800 & 0.00515400 \\
\hline $\mathrm{C}$ & 8.22114700 & -1.47958400 & 0.04396400 \\
\hline $\mathrm{H}$ & 6.55829500 & -2.85333400 & 0.04942700 \\
\hline $\mathrm{H}$ & 9.68780300 & 0.10453900 & 0.04312400 \\
\hline $\mathrm{H}$ & 8.97126800 & -2.26398300 & 0.06430700 \\
\hline $\mathrm{C}$ & -5.25943000 & -2.85003200 & -0.02703200 \\
\hline $\mathrm{H}$ & -6.12475800 & -3.20824600 & -0.58854500 \\
\hline $\mathrm{H}$ & -4.36174800 & -3.23854000 & -0.51091400 \\
\hline $\mathrm{H}$ & -5.31081800 & -3.24517200 & 0.99411300 \\
\hline $\mathrm{C}$ & 5.25943000 & 2.85003100 & -0.02707900 \\
\hline $\mathrm{H}$ & 5.31082000 & 3.24518300 & 0.99406200 \\
\hline $\mathrm{H}$ & 6.12475700 & 3.20823900 & -0.58859900 \\
\hline $\mathrm{H}$ & 4.36174700 & 3.23853500 & -0.51096300 \\
\hline $\mathrm{N}$ & -5.23210800 & -1.39973200 & -0.04648900 \\
\hline $\mathrm{N}$ & 5.23210800 & 1.39973200 & -0.04652000 \\
\hline
\end{tabular}


$\mathrm{N}$

$\begin{array}{lll}-0.44428900 & -0.45049200 & -0.01175900\end{array}$

$\mathrm{H}$

$-1.35902400$
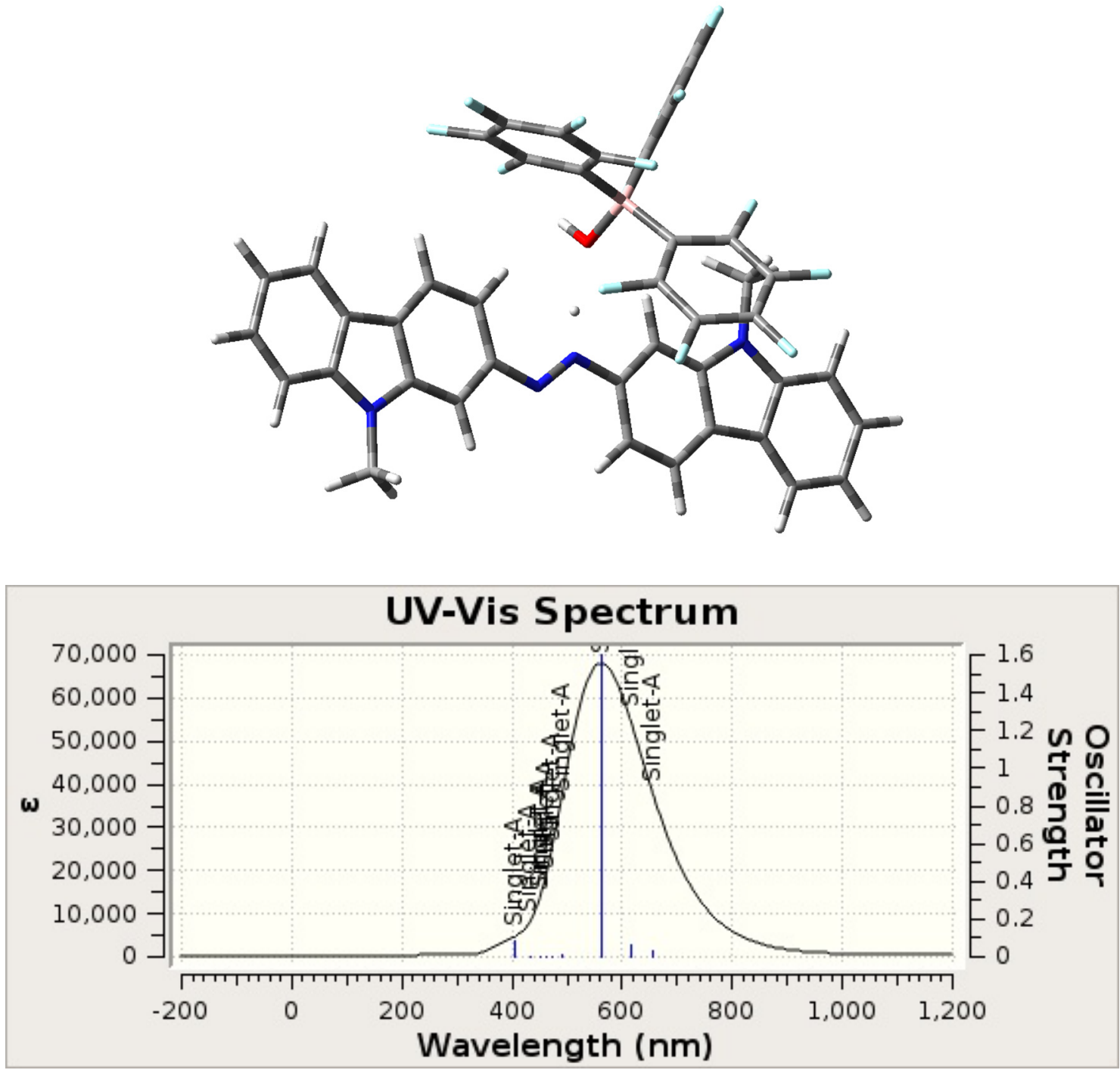

\begin{tabular}{|c|c|c|c|}
\hline TD State & Wavelength (nm) & Oscillator Strength & $\varepsilon$ \\
\hline 1 & 657.02 & 0.033 & 216835 \\
\hline 2 & 614.90 & 0.061 & 396986 \\
\hline 3 & 561.60 & 1.60 & 10453955 \\
\hline 4 & 489.95 & 0.012 & 1310 \\
\hline 5 & 472.62 & 0.00020 & 13756.93 \\
\hline 6 & 461.18 & 0.0021 & 3275 \\
\hline 7 & 452.21 & 0.00050 & \\
\hline
\end{tabular}




\begin{tabular}{|c|c|c|c|}
\hline 8 & 450.62 & 0.0028 & 18343 \\
\hline 9 & 432.02 & 0.00020 & 1310 \\
\hline 10 & 406.70 & 0.082 & 537175 \\
\hline
\end{tabular}

Functional and Basis Set: B3LYP/6-31G(d,p)

Charge: 0

Multiplicity: 1

Imaginary Frequencies: 0

Sum of electronic and thermal Free Energies: -3506.094310

Cartesian Coordinates:

C

C

C

C

C

C

C

$\mathrm{H}$

$\mathrm{H}$

$\mathrm{H}$

$\mathrm{N}$

C

C

C

C

$\mathrm{H}$

C
$-5.12884800$

$4.06127200-0.56282600$

$-3.70408300$

$3.86734500-0.57776100$

$-3.48828100$

$2.48756700-0.86056800$

$-2.21257800$

$1.94287600-0.93330700$

$-2.59637200$

$4.70865100-0.37334300$

$-1.13408200$

$2.81362800-0.70964800$

$-1.31519100$

$4.18958100-0.43654000$

$-2.74186100$

$5.76239100-0.15665100$

$-0.44650300$

$4.81327100-0.27113400$

$-2.02827000$

$0.89818000-1.15158400$

0.14637200

$2.23689100-0.76967600$

2.73375700

$1.02955600-1.10898100$

4.04034900

$0.59466500-1.20038300$

$\begin{array}{lll}2.44913000 & 2.37775200 & -0.74752700\end{array}$

$\begin{array}{lll}5.09227800 & 1.48598800 & -0.92311700\end{array}$

$\begin{array}{lll}4.24695900 & -0.43299400 & -1.48115200\end{array}$

3.49483300

$3.29443100-0.48677800$ 


\begin{tabular}{llll} 
C & 4.80013300 & 2.83895800 & -0.57256800 \\
H & 3.23489400 & 4.31123200 & -0.21717600 \\
C & 7.04225200 & 2.63194500 & -0.54960400 \\
C & 6.52111900 & 1.35608400 & -0.90807100 \\
C & 8.41935700 & 2.84767000 & -0.42702300 \\
C & 7.39773500 & 0.28763600 & -1.15536400 \\
C & 9.26320100 & 1.77118300 & -0.67983400 \\
H & 8.82259000 & 3.81241700 & -0.13862800 \\
C & 8.76332300 & 0.50340600 & -1.04072000 \\
H & 7.00970700 & -0.69004400 & -1.42461400 \\
H & 10.33627400 & 1.91134500 & -0.59100900 \\
H & 9.45705700 & -0.31023400 & -1.22489200 \\
C & -5.71383600 & 2.78939800 & -0.83048400 \\
C & -7.10300700 & 2.62204800 & -0.86762300 \\
C & -5.95460900 & 5.17464400 & -0.34534900 \\
C & -7.89483000 & 3.74367000 & -0.64771100 \\
H & -7.55477600 & 1.65384800 & -1.05380900 \\
C & -7.33183000 & 5.00966400 & -0.39040800 \\
H & -5.52084400 & 6.14917800 & -0.14136300 \\
H & -8.97532200 & 3.63859600 & -0.67116600 \\
H & -7.98491900 & 5.85993200 & -0.22247700 \\
C & 6.11775600 & 4.90009500 & 0.05831700 \\
H & 7.03026200 & 5.33078400 & -0.35947700 \\
\hline & -4.88108000 & 0.42592400 & -1.23883600
\end{tabular}




$\begin{array}{llll}\text { H } & -4.78176000 & -0.13742000 & -0.30496300 \\ \mathrm{H} & -5.86765400 & 0.24360200 & -1.66645200 \\ \mathrm{H} & -4.13305500 & 0.06089800 & -1.94604600 \\ \mathrm{~N} & 5.99272400 & 3.52045000 & -0.36796900 \\ \mathrm{~N} & -4.70780500 & 1.85477000 & -1.02597000 \\ \mathrm{~N} & 1.19789700 & 2.94707900 & -0.64714100 \\ \mathrm{H} & 1.93166800 & 0.33383300 & -1.31039500 \\ \mathrm{H} & 0.15068100 & 1.17464100 & -0.87811600 \\ \mathrm{~B} & -0.45082100 & -1.52344700 & -0.03389400 \\ \mathrm{C} & 0.99759500 & -2.26513700 & 0.32224700 \\ \mathrm{C} & 1.38255400 & -2.72936400 & 1.58328700 \\ \mathrm{C} & 1.97081500 & -2.43688600 & -0.66109600 \\ \mathrm{C} & 2.62863400 & -3.29183300 & 1.85728900 \\ \mathrm{C} & 3.23674000 & -2.96766800 & -0.43378900 \\ \mathrm{C} & 3.57263300 & -3.40303800 & 0.84188700 \\ \mathrm{C} & -1.47854200 & -2.64936900 & -0.68664800 \\ \mathrm{C} & -2.20133300 & -2.48176000 & -1.86884000 \\ \mathrm{C} & -1.69572800 & -3.88519700 & -0.06760100 \\ \mathrm{C} & -2.05262500 & -3.44175900 & -2.41374200 \\ \mathrm{C} & -2.52989300 & -4.87884800 & -0.57190700 \\ \mathrm{C} & -3.21548200 & -4.65744200 & -1.76233500 \\ \mathrm{C} & -1.07871300 & -0.72989600 & 1.27193500 \\ \mathrm{C} & -0.28725200 & 0.18819100 & 1.96846000 \\ \mathrm{C} & -2.39153600 & -0.81055800 & 1.73668700 \\ \mathrm{C} & -.72736300 & 0.94879200 & 3.04635600 \\ \mathrm{C} & & -0.06774300 & 2.81077500\end{array}$




$\begin{array}{llll}\text { C } & -2.04351900 & 0.81440700 & 3.47818800 \\ \text { O } & -0.19141700 & -0.41860000 & -1.03219800 \\ \text { H } & -0.02352400 & -0.79143200 & -1.90499900 \\ \text { F } & 4.78578500 & -3.91548600 & 1.08896300 \\ \text { F } & 2.93346600 & -3.71020200 & 3.09460400 \\ \text { F } & 4.14835100 & -3.01414000 & -1.42724600 \\ \text { F } & 1.73609100 & -2.03671100 & -1.94655300 \\ \text { F } & 0.54427800 & -2.63764900 & 2.63359700 \\ \text { F } & 0.99739400 & 0.39808400 & 1.58333900 \\ \text { F } & 0.09532500 & 1.81124000 & 3.66410800 \\ \text { F } & -2.49674600 & 1.53887500 & 4.50898600 \\ \text { F } & -4.16480700 & -0.18944200 & 3.19107300 \\ \text { F } & -3.30529400 & -1.60774600 & 1.13116700 \\ \text { F } & -1.09856500 & -4.16120300 & 1.10953500 \\ \text { F } & -2.68554700 & -6.04010700 & 0.07993800 \\ \text { F } & -4.02588700 & -5.59678600 & -2.26649400 \\ \text { F } & -3.71645200 & -3.19599700 & -3.55563300 \\ \text { F } & -2.13447500 & -1.31756500 & -2.58072300\end{array}$




\section{References}

1. Zhou, Y.-Y.; Hartline, D. R.; Steiman, T. J.; Fanwick, P. E.; Uyeda, C. Dinuclear Nickel Complexes in Five States of Oxidation Using a Redox-Active Ligand. Inorg. Chem. 2014, 53 (21), 11770-11777.

2. Bruker (2016). Apex3 v2016.9-0, Saint V8.34A, SAINT V8.37A, Bruker AXS Inc.: Madison (WI), USA, 2013/2014.

3. (a) SHELXTL suite of programs, Version 6.14, 2000-2003, Bruker Advanced X-ray Solutions, Bruker AXS Inc., Madison, Wisconsin: USA; (b) Sheldrick, G. M. Acta Crystallogr A. 2008, 64, 112122.

4. (a) Sheldrick, G. M. University of Göttingen, Germany, 2016; (b) Sheldrick, G. M. Acta Crystallogr Sect C Struct Chem. 2015, 71, 3-8.

5. Hübschle, C. B.; Sheldrick, G. M.; Dittrich, B. J. Appl. Crystallogr. 2011, 44, 1281-1284.

6. Miao, J.; Meng, B.; Liu, J.; Wang, L. An A-D-A'-D-A Type Small Molecule Acceptor with a Broad Absorption Spectrum for Organic Solar Cells. Chem. Commun. 2018, 54 (3), 303-306.

7. Bu, L.; Li, Y.; Wang, J.; Sun, M.; Zheng, M.; Liu, W.; Xue, S.; Yang, W. Synthesis and Piezochromic Luminescence of Aggregation-Enhanced Emission 9,10-Bis(N-Alkylcarbazol-2-Yl-Vinyl-

2)Anthracenes. Dyes and Pigments 2013, 99, 833-838.

8. Grimes, K; Gupte, A.; Aldrich, C.; Copper(II)-Catalyzed Conversion of Aryl/Heteroaryl Boronic Acids, Boronates, and Trifluoroborates into the Corresponding Azides: Substrate Scope and Limitations. Synthesis 2010, 9, 1441-1448.

9. Powers, I. G.; Andjaba, J. M.; Luo, X.; Mei, J.; Uyeda, C. Catalytic azoarene synthesis from aryl azides enabled by a dinuclear ni complex. J. Am. Chem. Soc. 2018, 140, 4110-4118.

10. Karakawa, M.; Aso, Y. Narrow-Optical-Gap $\pi$-Conjugated Small Molecules Based on Terminal Isoindigo and Thienoisoindigo Acceptor Units for Photovoltaic Application. RSC Adv. 2013, 3, 16259-16263.

11. Cartwright, L.; Yi, H.; Iraqi, A. Effect of Fluorination Pattern and Extent on the Properties of PCDTBT Derivatives. New J. Chem. 2016, 40, 1655-1662.

12. Mei, J.; Graham, K. R.; Stalder, R.; Reynolds, J. R. Synthesis of Isoindigo-Based Oligothiophenes for Molecular Bulk Heterojunction Solar Cells. Org. Lett. 2010, 12, 660-663.

13. Zhou, E.; Yamakawa, S.; Tajima, K.; Yang, C.; Hashimoto, K. Synthesis and Photovoltaic Properties of Diketopyrrolopyrrole-Based Donor-Acceptor Copolymers. Chem. Mater. 2009, 21, 4055-4061.

14. Reeves, B. D.; Grenier, C. R. G.; Argun, A. A.; Cirpan, A.; McCarley, T. D.; Reynolds, J. R. Spray Coatable Electrochromic Dioxythiophene Polymers with High Coloration Efficiencies. Macromol. 2004, 37, 7559-7569.

15. Hendriks, K. H.; Li, W.; Heintges, G. H. L.; van Pruissen, G. W. P.; Wienk, M. M.; Janssen, R. A. J. Homocoupling Defects in Diketopyrrolopyrrole-Based Copolymers and Their Effect on Photovoltaic Performance. J. Am. Chem. Soc. 2014, 136, 11128-11133.

16. Stéen, E. J. L.; Shalgunov, V.; Denk, C.; Mikula, H.; Kjær, A.; Kristensen, J. L.; Herth, M. M. Convenient Entry to 18F-Labeled Amines through the Staudinger Reduction. Eur. J. Org. Chem. 2019, (8), $1722-1725$.

17. Cianga, L.; Bendrea, A.-D.; Fifere, N.; Nita, L. E.; Doroftei, F.; Ag, D.; Seleci, M.; Timur, S.; Cianga, I. Fluorescent Micellar Nanoparticles by Self-Assembly of Amphiphilic, Nonionic and Water SelfDispersible Polythiophenes with "Hairy Rod" Architecture. RSC Adv. 2014, 4, 56385-56405.

18. Ni, Z.; Wang, H.; Dong, H.; Dang, Y.; Zhao, Q.; Zhang, X.; Hu, W. Mesopolymer Synthesis by LigandModulated Direct Arylation Polycondensation towards n-Type and Ambipolar Conjugated Systems. Nat. Chem. 2019, 11, 271-277.

19. Wang, L.; Pan, X.; Zhao, Y.; Chen, Y.; Zhang, W.; Tu, Y.; Zhang, Z.; Zhu, J.; Zhou, N.; Zhu, X. A Straightforward Protocol for the Highly Efficient Preparation of Main-Chain Azo Polymers Directly 
from Bisnitroaromatic Compounds by the Photocatalytic Process. Macromol. 2015, 48 (5), 12891295.

20. Gaussian 16, Revision A.03, Frisch, M. J.; Trucks, G. W.; Schlegel, H. B.; Scuseria, G. E.; Robb, M. A.; Cheeseman, J. R.; Scalmani, G.; Barone, V.; Petersson, G. A.; Nakatsuji, H.; Li, X.; Caricato, M.; Marenich, A. V.; Bloino, J.; Janesko, B. G.; Gomperts, R.; Mennucci, B.; Hratchian, H. P.; Ortiz, J. V.; Izmaylov, A. F.; Sonnenberg, J. L.; Williams-Young, D.; Ding, F.; Lipparini, F.; Egidi, F.; Goings, J.; Peng, B.; Petrone, A.; Henderson, T.; Ranasinghe, D.; Zakrzewski, V. G.; Gao, J.; Rega, N.; Zheng, G.; Liang, W.; Hada, M.; Ehara, M.; Toyota, K.; Fukuda, R.; Hasegawa, J.; Ishida, M.; Nakajima, T.; Honda, Y.; Kitao, O.; Nakai, H.; Vreven, T.; Throssell, K.; Montgomery, J. A., Jr.; Peralta, J. E.; Ogliaro, F.; Bearpark, M. J.; Heyd, J. J.; Brothers, E. N.; Kudin, K. N.; Staroverov, V. N.; Keith, T. A.; Kobayashi, R.; Normand, J.; Raghavachari, K.; Rendell, A. P.; Burant, J. C.; Iyengar, S. S.; Tomasi, J.; Cossi, M.; Millam, J. M.; Klene, M.; Adamo, C.; Cammi, R.; Ochterski, J. W.; Martin, R. L.; Morokuma, K.; Farkas, O.; Foresman, J. B.; Fox, D. J. Gaussian, Inc., Wallingford CT, 2016. 